Supporting Information

\title{
Regiodivergent Vinylogous-Cyclization Reactions of Cyclic $\alpha$-Amide Enone Acceptors: Synthesis of Highly Enantioenriched Heterobicyclic Structures
}

Yili Zhang, Xue Lu, Yichen Wang, Hui Xu, Ruoting Zhan, Weiwen Chen and Huicai Huang *

Research Center of Chinese Herbal Resource Science and Engineering, Key Laboratory of Chinese Medicinal Resource from Lingnan, Ministry of Education, Guangzhou University of Chinese Medicine, Guangzhou 510006, China. E-mail: huanghc@gzucm.edu.cn. 


\section{Table of Contents}

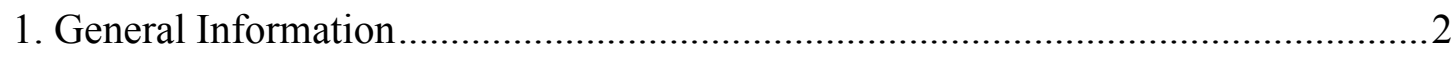

2. Solvents screening of the 1,2 -selective $\gamma$-addition cyclization reaction .................... 3

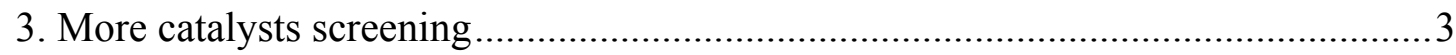

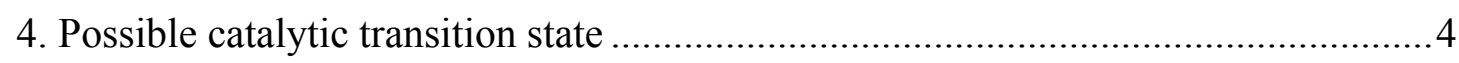

5. General procedure for the 1,4 -selective $\gamma$-addition cyclization reaction ...................5

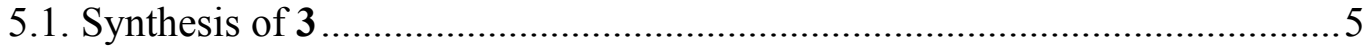

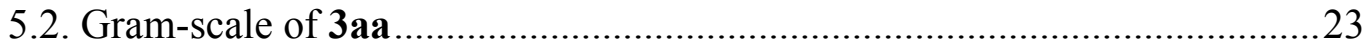

6. General procedure for the 1,2 -selective $\gamma$-addition cyclization reaction .................23

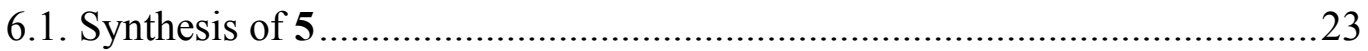

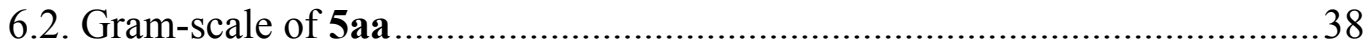

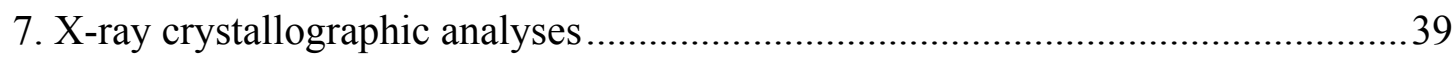

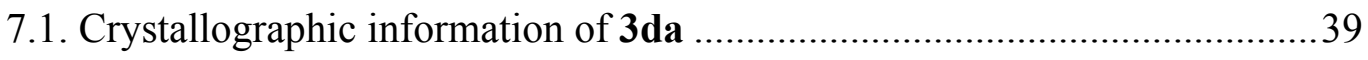

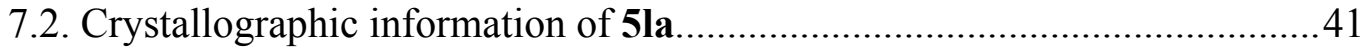

8. Transformation of vinylogous-cyclization products ............................................43

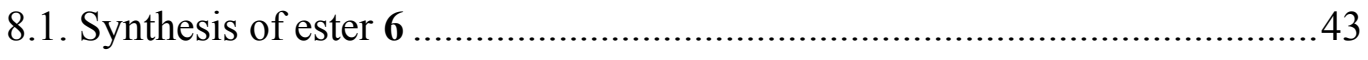

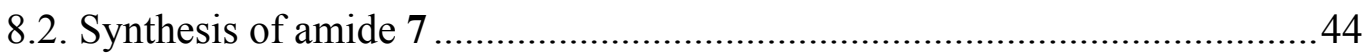

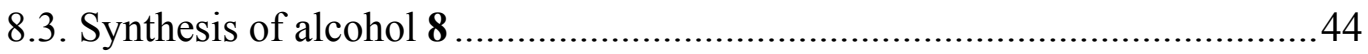

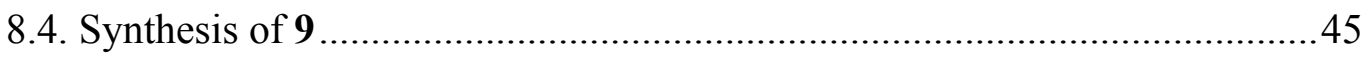

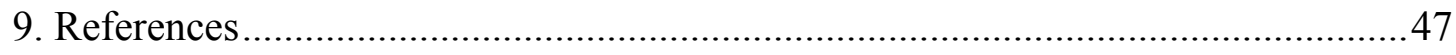

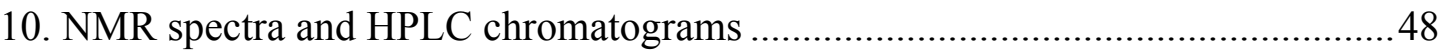




\section{General Information}

Proton nuclear magnetic resonance $\left({ }^{1} \mathrm{H}\right.$ NMR) spectra and carbon nuclear magnetic resonance $\left({ }^{13} \mathrm{C}\right.$ NMR) spectra were recorded on a Bruker AV-400 spectrometer $(400 \mathrm{MHz}$ and $100 \mathrm{MHz})$. Chemical shifts for protons are reported in parts per million downfield from tetramethylsilane and are referenced to residual protium in the NMR solvent $\left(\mathrm{CDCl}_{3}: \delta 7.26\right)$ Chemical shifts for carbon are reported in parts per million downfield from tetramethylsilane and are referenced to the carbon resonances of the solvent $\left(\mathrm{CDCl}_{3}: \delta 77.16\right)$. Data are represented as follows: chemical shift, integration, multiplicity $(\mathrm{br}=$ broad, $\mathrm{s}=$ singlet, $\mathrm{d}=$ doublet, $\mathrm{t}=$ triplet, $\mathrm{q}=$ quartet, $\mathrm{m}=$ multiplet), coupling constants in Hertz $(\mathrm{Hz})$. High resolution mass spectrometry (ESI) were carried out using a TSQ Quantum Access, Thermo Fisher, USA. Optical rotations were measured on an Autopol III automatic polarimeter (Rudolph Research analytical). High performance liquid chromatography (HPLC) was performed on an Agilent 1260 Infinity II series chromatographs using chiral columns (DAICEL CHIRALPAK) as noted.

Starting Materials. All solvents and organic reagents were from commercial sources (Adamas-beta ${ }^{\circledR}$ ) and used without purification unless otherwise noted. 


\section{Solvents screening of the 1,2 -selective $\gamma$-addition cyclization reaction $^{[\mathrm{a}]}$}

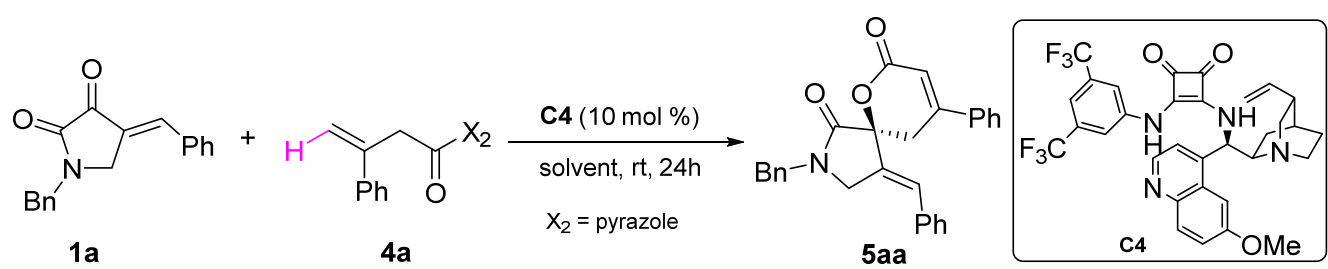

\begin{tabular}{cccc}
\hline entry & solvent & yield $(\%)^{[\mathrm{b}]}$ & ee $(\%)^{[\mathrm{c}]}$ \\
\hline & $\mathrm{CH}_{2} \mathrm{Cl}_{2}$ & 98 & 98 \\
1 & $\mathrm{EtOH}$ & 57 & 92 \\
2 & $\mathrm{THF}$ & 99 & 99 \\
3 & $\mathrm{EtOAc}$ & 93 & 98 \\
4 & $\mathrm{DCE}$ & 67 & 95 \\
5 & $\mathrm{CHCl}_{3}$ & 79 & 96 \\
6 & $\mathrm{MeCN}_{7}$ & 98 & 96 \\
8 & toluene & 80 & 97 \\
\hline
\end{tabular}

[a] Unless otherwise noted, all reactions were carried out with 1.0 equiv of $1 \mathbf{a}(0.1 \mathrm{mmol}), 2.0$ equiv of $4 \mathbf{a}(0.2$ $\mathrm{mmol})$, catalyst $\mathbf{C 4}(0.01 \mathrm{mmol}, 10 \mathrm{~mol} \%)$ in the specified solvent $(1.0 \mathrm{~mL})$ at room temperature for $24 \mathrm{~h}$. [b] Yield of the isolated product 5aa after column chromatography. [c] Determined by HPLC analysis using a chiral column.

\section{More Catalysts screening}<smiles>C=CC1CC2CCC1N2C(OCc1ccccc1)c1ccnc2ccc(OC)cc12</smiles>

C7<smiles>C=CC1CC2CCC1N2Cc1ccnc2ccc(O)cc12</smiles><smiles></smiles>

C8<smiles>[B][C@H](c1ccnc2ccc(O)cc12)C1C2C=CC1N(C)CC2</smiles><smiles>C=CC1C2CCN(C2)C1C(O)c1ccnc2ccc(OC)cc12</smiles>

C10<smiles>C=CC1CC2CCN1C(C(O)c1ccnc3ccc(O)cc13)C2</smiles><smiles>O=C1C(=O)N(Cc2ccccc2)C/C1=C\c1ccccc1</smiles>

$1 a$<smiles>C=CC1CC2CCN1C2C(O)c1ccnc2ccc(OC)cc12</smiles>

c9<smiles>C=CC1CC2CCN1C2[C@H](O)c1ccnc2ccc(O)cc12</smiles>

C13<smiles>Cc1cc(C)n(C(=O)C[C@H]2OC3=C(CN(Cc4ccccc4)C3=O)[C@H](c3ccccc3)[C@H]2c2ccccc2)n1</smiles>

$3 a a$

\begin{tabular}{ccccc}
\hline Catalyst $^{a}$ & time & yield $(\%)^{b}$ & $\mathrm{dr}^{c}$ & ee $(\%)^{d}$ \\
\hline C7 & $48 \mathrm{~h}$ & 90 & $47: 53$ & $-12 /-18$
\end{tabular}




\begin{tabular}{ccccc} 
C8 & $48 \mathrm{~h}$ & 94 & $59: 41$ & $17 / 28$ \\
C9 & $1 \mathrm{~h}$ & 99 & $62: 38$ & $36 /-25$ \\
C10 & $1 \mathrm{~h}$ & 99 & $61: 39$ & $-44 / 52$ \\
C11 & $43 \mathrm{~h}$ & 99 & $52: 48$ & $-85 / 3$ \\
C12 & $43 \mathrm{~h}$ & 99 & $52: 48$ & $78 /-2$ \\
C13 & $2 \mathrm{~h}$ & 99 & $60: 40$ & $40 /-20$ \\
C14 & $72 \mathrm{~h}$ & 82 & $48: 52$ & $52 /-42$ \\
\hline
\end{tabular}

${ }^{a}$ General reaction conditions: 1a $(0.05 \mathrm{mmol}), 2 \mathrm{a}(0.06 \mathrm{mmol})$, catalyst $\mathbf{C}(0.005 \mathrm{mmol}, 10 \mathrm{~mol} \%)$ in DCM $(0.5$ $\mathrm{mL}$ ) at room temperature. ${ }^{b}$ Isolated yield. ${ }^{c}$ Determined by crude ${ }^{1} \mathrm{H}$ NMR analysis. ${ }^{d}$ Determined by chiral HPLC analysis.

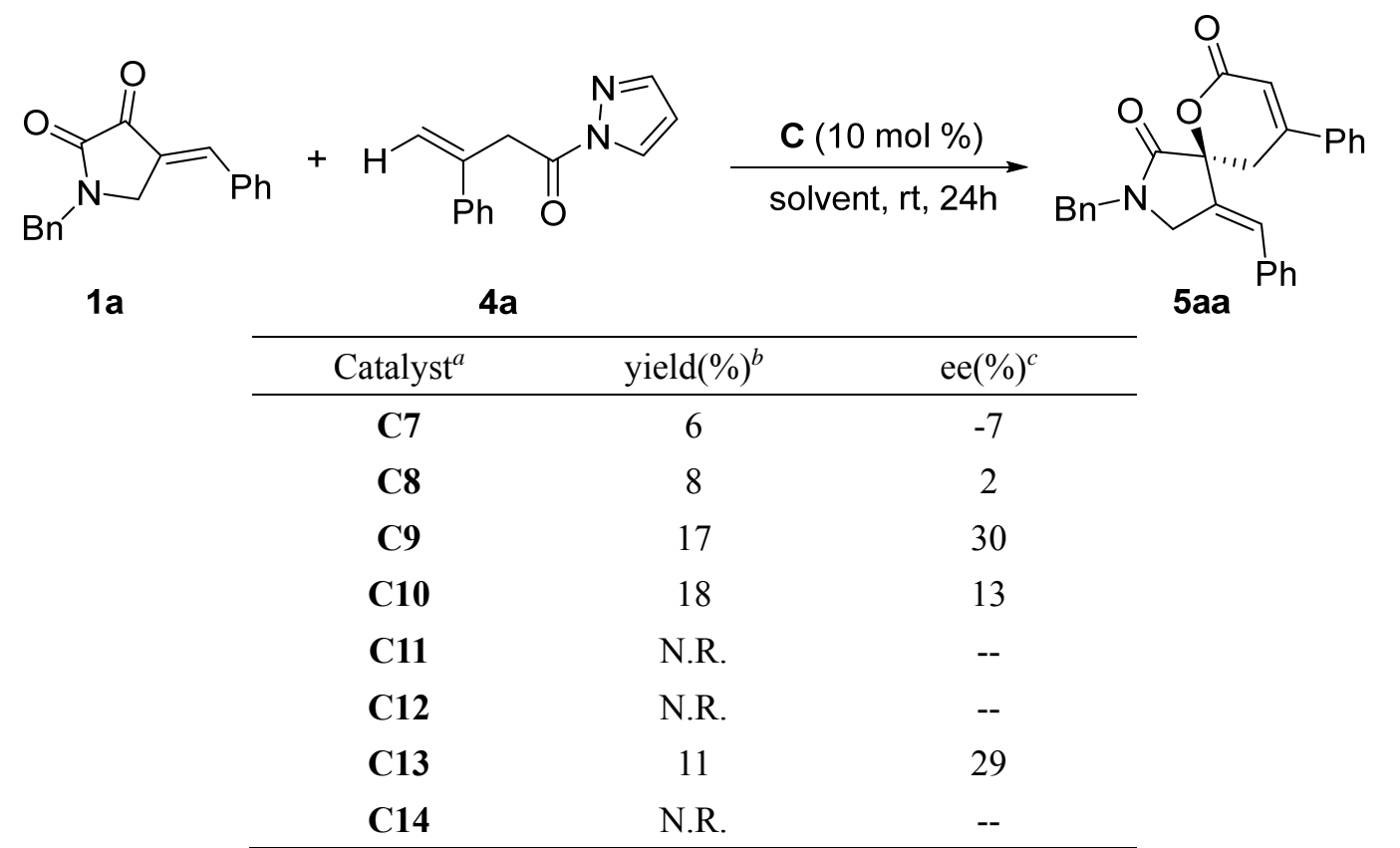

${ }^{a}$ General reaction conditions: 1a $(0.1 \mathrm{mmol}), \mathbf{4 a}(0.2 \mathrm{mmol})$, catalyst $\mathbf{C}(0.01 \mathrm{mmol}, 10 \mathrm{~mol} \%)$ in DCM $(1.0 \mathrm{~mL})$ at room temperature for $24 \mathrm{~h} .{ }^{b}$ Isolated yield. ${ }^{c}$ Determined by chiral HPLC analysis.

\section{Possible catalytic transition state}

Based on the experimental results and recent studies, we proposed a possible transition state to explain the 1,4-selective $\gamma$-addition cyclization reaction of cyclic $\alpha$-amide enones and 3-butenyl $N$-acylpyrazoles. The $\beta, \gamma$-position of the 3-butenyl- $N$-acylpyrazole could be activated by a part of the tertiary amine catalyst to form a dienolate in situ, while the cyclic $\alpha$-amide enone activated by two hydrogen atoms of squaramide through hydrogen-bonding to react with the $\beta, \gamma$-position of the dienolate. 

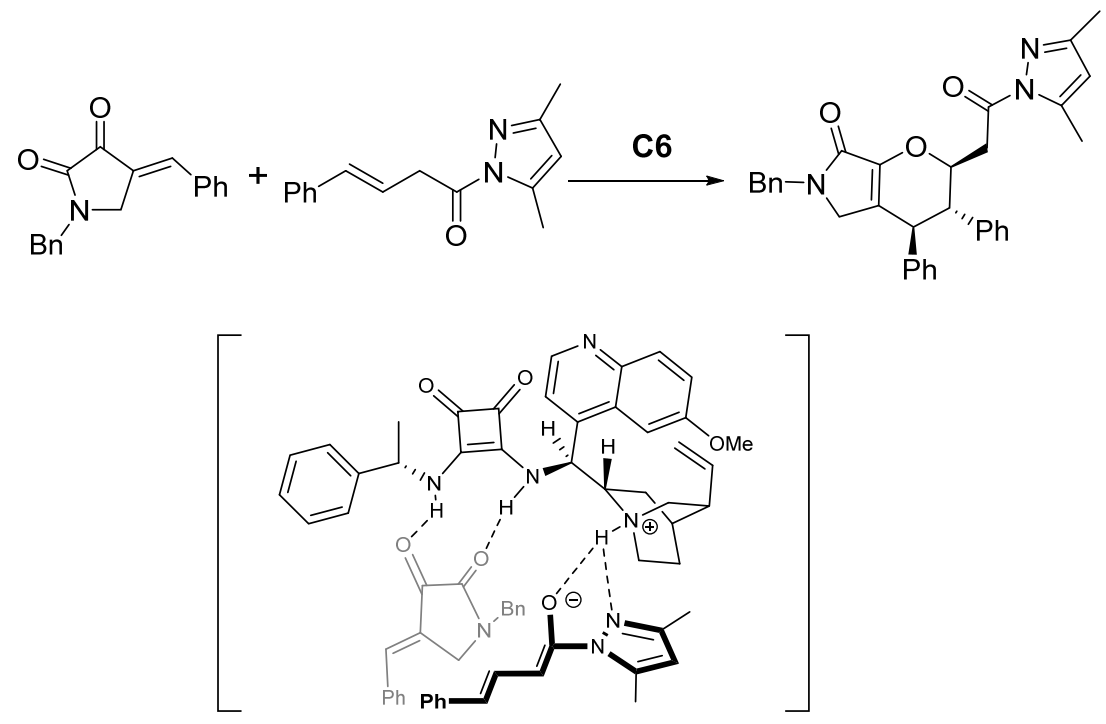

\section{General procedure for the 1,4 -selective $\gamma$-addition cyclization reaction}

\subsection{Synthesis of 3}

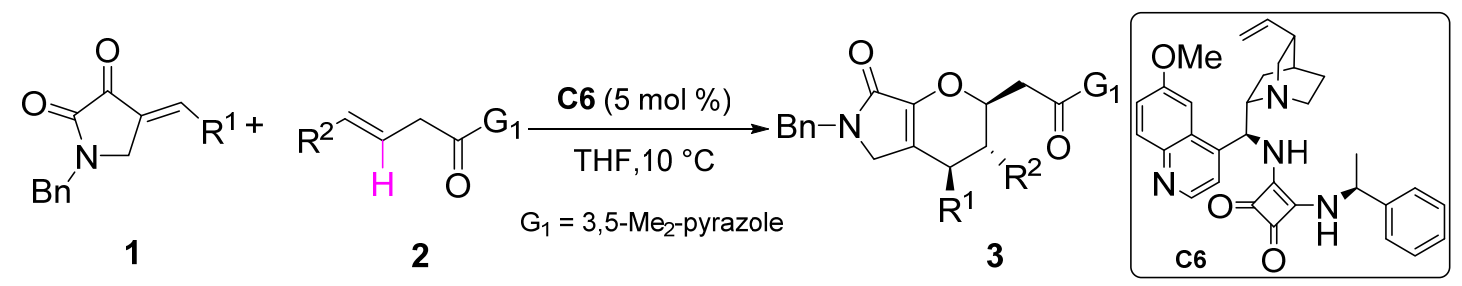

Unsaturated ketone 1 (0.2 mmol, 1.0 equiv.) and catalyst C6 (0.01 mmol, 0.05 equiv.) in THF ( $2 \mathrm{~mL})$ were cooled to $10^{\circ} \mathrm{C}$, then $N$-acylpyrazole $2(0.24$ mmol 1.2 equiv.) was added. The reaction mixture was stirred at $10^{\circ} \mathrm{C}$ for $24-48$ hours. After removal of the solvent under reduced pressure, the reaction mixture was purified by flash chromatography on silica gel (hexane/EtOAc $=3 / 1$ ) to give the pure product 3 .

(2S,3S,4S)-6-benzyl-2-(2-(3,5-dimethyl-1H-pyrazol-1-yl)-2-oxoethyl)-3,4-diphenyl $-3,4,5,6$-tetrahydropyrano[2,3-c]pyrrol-7(2H)-one (3aa)<smiles></smiles> 
White solid, $101.4 \mathrm{mg}, 98 \%$ yield, $\mathrm{mp}=188.5-188.7{ }^{\circ} \mathrm{C},[\alpha] \mathrm{D}^{25}=-48.4(\mathrm{c} 0.965$, $\left.\mathrm{CH}_{2} \mathrm{Cl}_{2}\right), 96: 4 \mathrm{dr},>99 \%$ ee.

${ }^{1} \mathrm{H}$ NMR (400 MHz, $\left.\mathrm{CDCl}_{3}\right) \delta 7.31$ (q, $\left.J=7.2 \mathrm{~Hz}, 3 \mathrm{H}\right), 7.22(\mathrm{~d}, J=7.5 \mathrm{~Hz}, 2 \mathrm{H}), 7.14$ $(\mathrm{s}, 6 \mathrm{H}), 6.96(\mathrm{~d}, J=6.4 \mathrm{~Hz}, 2 \mathrm{H}), 6.85(\mathrm{~s}, 2 \mathrm{H}), 5.90(\mathrm{~s}, 1 \mathrm{H}), 5.11(\mathrm{t}, J=9.1 \mathrm{~Hz}, 1 \mathrm{H})$, $4.89(\mathrm{~d}, J=15.0 \mathrm{~Hz}, 1 \mathrm{H}), 4.35(\mathrm{~d}, J=15.0 \mathrm{~Hz}, 1 \mathrm{H}), 3.84(\mathrm{~d}, J=10.0 \mathrm{~Hz}, 1 \mathrm{H}), 3.64$ $(\mathrm{dd}, J=17.3,7.9 \mathrm{~Hz}, 1 \mathrm{H}), 3.54(\mathrm{~d}, J=18.2 \mathrm{~Hz}, 1 \mathrm{H}), 3.41(\mathrm{~d}, J=18.1 \mathrm{~Hz}, 1 \mathrm{H}), 3.17-$ $3.07(\mathrm{~m}, 2 \mathrm{H}), 2.48(\mathrm{~s}, 3 \mathrm{H}), 2.18(\mathrm{~s}, 3 \mathrm{H})$.

${ }^{13} \mathrm{C}$ NMR $\left(100 \mathrm{MHz}, \mathrm{CDCl}_{3}\right) \delta 170.2,165.0,151.8,146.1,144.2,139.7,138.7,137.1$, $128.7,128.5,128.0,127.6,127.3,127.2,124.0,111.2,77.9,52.9,47.7,47.3,46.5$, $38.8,14.5,13.8$.

HRMS (ESI) $m / z$ calculated for $\mathrm{C}_{33} \mathrm{H}_{32} \mathrm{~N}_{3} \mathrm{O}_{3}{ }^{+}[\mathrm{M}+\mathrm{H}]^{+}:$518.2438, found 518.2442.

HPLC analysis: (IA column, Hexane:2-propanol $=80: 20$, flow rate $=0.4 \mathrm{~mL} / \mathrm{min}$, wavelength $=254 \mathrm{~nm}$ ): $R \mathrm{t}=25.08$ (major), 22.64 (minor).

(2S,3S,4S)-6-benzyl-2-(2-(3,5-dimethyl-1H-pyrazol-1-yl)-2-oxoethyl)-4-(3-fluorop henyl)-3-phenyl-3,4,5,6-tetrahydropyrano[2,3-c]pyrrol-7(2H)-one (3ba)

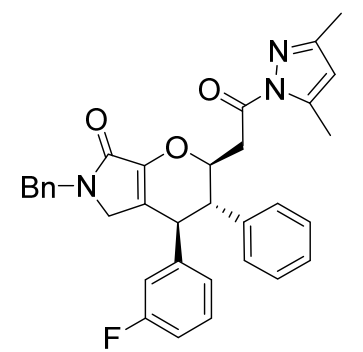

White solid, $103.3 \mathrm{mg}, 96 \%$ yield, $\mathrm{mp}=180.5-181.4{ }^{\circ} \mathrm{C},[\alpha] \mathrm{D}^{25}=-42.9$ (c 1.013, $\left.\mathrm{CH}_{2} \mathrm{Cl}_{2}\right), 94: 6 \mathrm{dr},>99 \%$ ee.

${ }^{1} \mathrm{H}$ NMR (400 MHz, $\left.\mathrm{CDCl}_{3}\right) \delta 7.35-7.27(\mathrm{~m}, 3 \mathrm{H}), 7.24-7.13(\mathrm{~m}, 5 \mathrm{H}), 7.13-7.07$ $(\mathrm{m}, 1 \mathrm{H}), 6.97(\mathrm{~d}, J=6.5 \mathrm{~Hz}, 2 \mathrm{H}), 6.87-6.80(\mathrm{~m}, 1 \mathrm{H}), 6.60(\mathrm{dd}, J=22.9,8.3 \mathrm{~Hz}, 2 \mathrm{H})$, 5.89 (s, 1H), 5.09 (t, $J=7.8 \mathrm{~Hz}, 1 \mathrm{H}), 4.89$ (d, $J=14.9 \mathrm{~Hz}, 1 \mathrm{H}), 4.36$ (d, $J=14.9 \mathrm{~Hz}$, $1 \mathrm{H}), 3.85(\mathrm{~d}, J=9.9 \mathrm{~Hz}, 1 \mathrm{H}), 3.62(\mathrm{dd}, J=17.4,7.8 \mathrm{~Hz}, 1 \mathrm{H}), 3.55(\mathrm{~d}, J=18.1 \mathrm{~Hz}$, 1H), 3.40 (d, J=18.0 Hz, 1H), $3.17-3.08$ (m, 2H), 2.47 (s, 3H), 2.17 (s, 3H).

${ }^{13} \mathrm{C}$ NMR $\left(100 \mathrm{MHz}, \mathrm{CDCl}_{3}\right) \delta 170.1,164.8,164.0,161.5,151.9,146.4,144.1,142.3$, $142.3,138.3,137.0,130.1,130.1,128.8,128.7,128.3,128.0,127.6,127.6,123.7$, $123.1,115.0,114.8,114.3,114.1,111.2,77.9,52.6,47.4,47.1,46.5,38.7,14.5,13.8$.

HRMS (ESI) $m / z$ calculated for $\mathrm{C}_{33} \mathrm{H}_{31} \mathrm{FN}_{3} \mathrm{O}_{3}{ }^{+}[\mathrm{M}+\mathrm{H}]^{+}:$: 536.2344, found 536.2340.

HPLC analysis: (IA column, Hexane:2-propanol $=80: 20$, flow rate $=0.4 \mathrm{~mL} / \mathrm{min}$, wavelength $=254 \mathrm{~nm}$ ): $R \mathrm{t}=32.21$ (major), 28.34 (minor). 
(2S,3S,4S)-6-benzyl-4-(3-chlorophenyl)-2-(2-(3,5-dimethyl-1H-pyrazol-1-yl)-2-ox oethyl)-3-phenyl-3,4,5,6-tetrahydropyrano[2,3-c]pyrrol-7(2H)-one (3ca)<smiles></smiles>

White solid, $108.6 \mathrm{mg}, 98 \%$ yield, $\mathrm{mp}=184.2-184.7{ }^{\circ} \mathrm{C},[\alpha] \mathrm{D}^{25}=-50.6(c$ 1.007, $\left.\mathrm{CH}_{2} \mathrm{Cl}_{2}\right), 94: 6 \mathrm{dr},>99 \%$ ee.

${ }^{1} \mathrm{H}$ NMR (400 MHz, $\left.\mathrm{CDCl}_{3}\right) \delta 7.36-7.27(\mathrm{~m}, 3 \mathrm{H}), 7.24-7.14(\mathrm{~m}, 5 \mathrm{H}), 7.09$ (d, J = $14.8 \mathrm{~Hz}, 2 \mathrm{H}), 6.97(\mathrm{~s}, 2 \mathrm{H}), 6.85(\mathrm{~s}, 1 \mathrm{H}), 6.72(\mathrm{~s}, 1 \mathrm{H}), 5.89(\mathrm{~s}, 1 \mathrm{H}), 5.09(\mathrm{~s}, 1 \mathrm{H}), 4.89$ (d, $J=14.4 \mathrm{~Hz}, 1 \mathrm{H}), 4.36(\mathrm{~d}, J=14.2 \mathrm{~Hz}, 1 \mathrm{H}), 3.83(\mathrm{~d}, J=7.4 \mathrm{~Hz}, 1 \mathrm{H}), 3.69-3.60$ (m, 1H), $3.55(\mathrm{~d}, J=18.4 \mathrm{~Hz}, 1 \mathrm{H}), 3.40(\mathrm{~d}, J=17.3 \mathrm{~Hz}, 1 \mathrm{H}), 3.12(\mathrm{~d}, J=14.7 \mathrm{~Hz}$, $2 \mathrm{H}), 2.47$ (s, 3H), 2.17 (s, 3H).

${ }^{13} \mathrm{C}$ NMR (100 MHz, $\left.\mathrm{CDCl}_{3}\right) \delta 170.1,164.8,151.9,146.5,144.1,141.8,138.2,137.0$, $134.4,129.9,128.8,128.8,128.0,127.6,127.6,127.5,126.3,123.0,111.2,77.9,52.7$, $47.4,47.1,46.5,38.7,14.5,13.8$.

HRMS (ESI) $m / z$ calculated for $\mathrm{C}_{33} \mathrm{H}_{31} \mathrm{ClN}_{3} \mathrm{O}_{3}{ }^{+}[\mathrm{M}+\mathrm{H}]^{+}:$552.2049, found 552.2048.

HPLC analysis: (IA column, Hexane:2-propanol $=80: 20$, flow rate $=0.4 \mathrm{~mL} / \mathrm{min}$, wavelength $=254 \mathrm{~nm}$ ): $R \mathrm{t}=26.94$ (major), 23.20 (minor).

(2S,3S,4S)-6-benzyl-4-(3-bromophenyl)-2-(2-(3,5-dimethyl-1H-pyrazol-1-yl)-2-ox oethyl)-3-phenyl-3,4,5,6-tetrahydropyrano[2,3-c]pyrrol-7(2H)-one (3da)<smiles></smiles>

White solid, $116.6 \mathrm{mg}, 98 \%$ yield, $\mathrm{mp}=169.0-169.8^{\circ} \mathrm{C},[\alpha] \mathrm{D}^{25}=-55.3($ c 1.156 , $\left.\mathrm{CH}_{2} \mathrm{Cl}_{2}\right), 95: 5 \mathrm{dr},>99 \%$ ee.

${ }^{1} \mathrm{H}$ NMR (400 MHz, $\left.\mathrm{CDCl}_{3}\right) \delta 7.37-7.26(\mathrm{~m}, 4 \mathrm{H}), 7.19(\mathrm{dd}, J=12.6,7.6 \mathrm{~Hz}, 5 \mathrm{H})$, $7.05-6.90(\mathrm{~m}, 4 \mathrm{H}), 6.73(\mathrm{~d}, J=6.7 \mathrm{~Hz}, 1 \mathrm{H}), 5.88(\mathrm{~s}, 1 \mathrm{H}), 5.07(\mathrm{t}, J=7.9 \mathrm{~Hz}, 1 \mathrm{H})$, $4.88(\mathrm{~d}, J=14.9 \mathrm{~Hz}, 1 \mathrm{H}), 4.35(\mathrm{~d}, J=15.0 \mathrm{~Hz}, 1 \mathrm{H}), 3.79(\mathrm{~d}, J=9.9 \mathrm{~Hz}, 1 \mathrm{H}), 3.61$ (dd, $J=17.3,7.9 \mathrm{~Hz}, 1 \mathrm{H}), 3.53(\mathrm{~d}, J=18.2 \mathrm{~Hz}, 1 \mathrm{H}), 3.38(\mathrm{~d}, J=18.1 \mathrm{~Hz}, 1 \mathrm{H}), 3.15-3.04$ (m, 2H), $2.46(\mathrm{~s}, 3 \mathrm{H}), 2.16(\mathrm{~s}, 3 \mathrm{H})$. 
${ }^{13} \mathrm{C} \mathrm{NMR}\left(100 \mathrm{MHz}, \mathrm{CDCl}_{3}\right) \delta 170.1,164.8,151.9,146.5,144.2,142.1,138.2,136.9$, $130.9,130.4,130.1,128.9,128.8,128.0,127.6,127.6,126.8,122.9,122.6,111.3$, $77.9,52.7,47.4,47.1,46.5,38.7,14.5,13.8$.

HRMS (ESI) $m / z$ calculated for $\mathrm{C}_{33} \mathrm{H}_{31} \mathrm{BrN}_{3} \mathrm{O}_{3}{ }^{+}[\mathrm{M}+\mathrm{H}]^{+}:$596.1543, found 596.1542.

HPLC analysis: (IA column, Hexane:2-propanol $=70: 30$, flow rate $=0.3 \mathrm{~mL} / \mathrm{min}$, wavelength $=254 \mathrm{~nm}$ ): $R \mathrm{t}=25.72$ (major), 22.80 (minor).

(2S,3S,4S)-6-benzyl-2-(2-(3,5-dimethyl-1H-pyrazol-1-yl)-2-oxoethyl)-3-phenyl-4-( m-tolyl)-3,4,5,6-tetrahydropyrano[2,3-c]pyrrol-7(2H)-one (3ea)

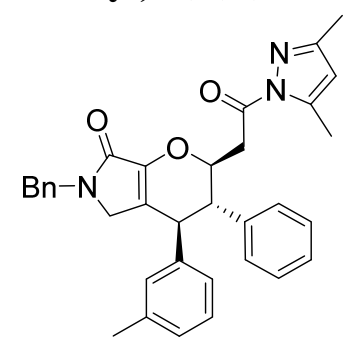

White solid, $104.3 \mathrm{mg}, 98 \%$ yield, $\mathrm{mp}=177.0-178.3{ }^{\circ} \mathrm{C},[\alpha] \mathrm{D}^{25}=-54.8(\mathrm{c} 1.047$, $\left.\mathrm{CH}_{2} \mathrm{Cl}_{2}\right), 97: 3 \mathrm{dr},>99 \%$ ee.

${ }^{1} \mathrm{H}$ NMR $\left(400 \mathrm{MHz}, \mathrm{CDCl}_{3}\right) \delta 7.30(\mathrm{dt}, J=8.7,4.7 \mathrm{~Hz}, 3 \mathrm{H}), 7.22(\mathrm{~d}, J=7.3 \mathrm{~Hz}, 3 \mathrm{H})$, $7.16(\mathrm{~d}, J=7.2 \mathrm{~Hz}, 2 \mathrm{H}), 6.99(\mathrm{dt}, J=26.2,7.8 \mathrm{~Hz}, 4 \mathrm{H}), 6.67-6.58(\mathrm{~m}, 2 \mathrm{H}), 5.90$ (s, $1 \mathrm{H}), 5.10$ (t, $J=7.8 \mathrm{~Hz}, 1 \mathrm{H}), 4.87(\mathrm{~d}, J=14.9 \mathrm{~Hz}, 1 \mathrm{H}), 4.38(\mathrm{~d}, J=14.9 \mathrm{~Hz}, 1 \mathrm{H})$, $3.80(\mathrm{~d}, J=9.8 \mathrm{~Hz}, 1 \mathrm{H}), 3.64(\mathrm{dd}, J=17.3,8.0 \mathrm{~Hz}, 1 \mathrm{H}), 3.54(\mathrm{~d}, J=18.1 \mathrm{~Hz}, 1 \mathrm{H})$, $3.41(\mathrm{~d}, J=18.1 \mathrm{~Hz}, 1 \mathrm{H}), 3.19-3.05(\mathrm{~m}, 2 \mathrm{H}), 2.48(\mathrm{~s}, 3 \mathrm{H}), 2.19(\mathrm{~d}, J=7.7 \mathrm{~Hz}, 6 \mathrm{H})$.

${ }^{13} \mathrm{C}$ NMR $\left(100 \mathrm{MHz}, \mathrm{CDCl}_{3}\right) \delta 170.3,165.0,151.8,146.0,144.1,139.6,138.8,138.1$, $137.1,128.7,128.7,128.3,128.0,127.9,127.6,127.3,125.2,124.2,111.2,77.9,52.8$, $47.6,47.3,46.5,38.8,21.3,14.5,13.7$.

HRMS (ESI) $m / z$ calculated for $\mathrm{C}_{34} \mathrm{H}_{34} \mathrm{~N}_{3} \mathrm{O}_{3}{ }^{+}[\mathrm{M}+\mathrm{H}]^{+}:$532.2595, found 532.2594.

HPLC analysis: (IC column, Hexane:2-propanol $=40: 60$, flow rate $=0.5 \mathrm{~mL} / \mathrm{min}$, wavelength $=254 \mathrm{~nm}$ ): $R \mathrm{t}=59.37$ (major), 45.13 (minor).

(2S,3S,4S)-6-benzyl-2-(2-(3,5-dimethyl-1H-pyrazol-1-yl)-2-oxoethyl)-3-phenyl-4-( 4-(trifluoromethyl)phenyl)-3,4,5,6-tetrahydropyrano[2,3-c]pyrrol-7(2H)-one (3fa)<smiles></smiles> 
White solid, $104.3 \mathrm{mg}, 89 \%$ yield, $\mathrm{mp}=90.6-92.6{ }^{\circ} \mathrm{C},[\alpha] \mathrm{D}^{25}=-38.2($ c 0.986 , $\mathrm{CH}_{2} \mathrm{Cl}_{2}$ ), $94: 6 \mathrm{dr}, 98 \%$ ee.

${ }^{1} \mathrm{H}$ NMR $\left(400 \mathrm{MHz}, \mathrm{CDCl}_{3}\right) \delta 7.40(\mathrm{~d}, J=6.5 \mathrm{~Hz}, 2 \mathrm{H}), 7.34-7.26(\mathrm{~m}, 3 \mathrm{H}), 7.19(\mathrm{dd}$, $J=16.1,7.2 \mathrm{~Hz}, 5 \mathrm{H}), 6.96(\mathrm{~s}, 4 \mathrm{H}), 5.90(\mathrm{~s}, 1 \mathrm{H}), 5.11(\mathrm{~s}, 1 \mathrm{H}), 4.88(\mathrm{~d}, J=14.6 \mathrm{~Hz}$, $1 \mathrm{H}), 4.36(\mathrm{~d}, J=14.7 \mathrm{~Hz}, 1 \mathrm{H}), 3.94(\mathrm{~d}, J=8.9 \mathrm{~Hz}, 1 \mathrm{H}), 3.59(\mathrm{dt}, J=27.4,13.3 \mathrm{~Hz}$, 2H), 3.39 (d, $J=17.6 \mathrm{~Hz}, 1 \mathrm{H}), 3.12(\mathrm{~d}, J=17.1 \mathrm{~Hz}, 2 \mathrm{H}), 2.46(\mathrm{~s}, 3 \mathrm{H}), 2.16(\mathrm{~s}, 3 \mathrm{H})$.

${ }^{13} \mathrm{C}$ NMR $\left(100 \mathrm{MHz}, \mathrm{CDCl}_{3}\right) \delta 170.1,164.8,152.0,146.6,144.2,143.9,138.0,136.9$, 125.6, 125.5, 125.3, 122.7, 122.6, 111.3, 77.9, 52.7, 47.5, 47.1, 46.5, 38.6, 14.5, 13.8.

HRMS (ESI) $m / z$ calculated for $\mathrm{C}_{34} \mathrm{H}_{31} \mathrm{~F}_{3} \mathrm{~N}_{3} \mathrm{O}_{3}{ }^{+}[\mathrm{M}+\mathrm{H}]^{+}:$: 586.2312, found 586.2311.

HPLC analysis: (IC column, Hexane:2-propanol $=40: 60$, flow rate $=0.5 \mathrm{~mL} / \mathrm{min}$, wavelength $=254 \mathrm{~nm}$ ): $R \mathrm{t}=36.03$ (major), 30.01 (minor).

(2S,3S,4S)-6-benzyl-4-(4-chlorophenyl)-2-(2-(3,5-dimethyl-1H-pyrazol-1-yl)-2-ox oethyl)-3-phenyl-3,4,5,6-tetrahydropyrano[2,3-c]pyrrol-7(2H)-one (3ga)

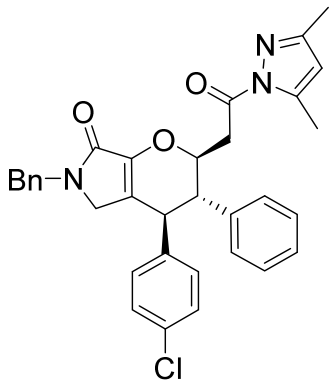

White solid, $105.8 \mathrm{mg}, 96 \%$ yield, $\mathrm{mp}=172.1-172.9{ }^{\circ} \mathrm{C},[\alpha] \mathrm{D}^{25}=-52.3($ c 1.044, $\left.\mathrm{CH}_{2} \mathrm{Cl}_{2}\right), 98: 2 \mathrm{dr},>99 \%$ ee.

${ }^{1} \mathrm{H}$ NMR $\left(400 \mathrm{MHz}, \mathrm{CDCl}_{3}\right) \delta 7.29(\mathrm{~d}, J=6.9 \mathrm{~Hz}, 3 \mathrm{H}), 7.18(\mathrm{dd}, J=17.0,7.4 \mathrm{~Hz}$, $5 \mathrm{H}), 7.10(\mathrm{~d}, J=7.7 \mathrm{~Hz}, 2 \mathrm{H}), 6.99-6.90(\mathrm{~m}, 2 \mathrm{H}), 6.76(\mathrm{~d}, J=7.6 \mathrm{~Hz}, 2 \mathrm{H}), 5.88(\mathrm{~s}$, $1 \mathrm{H}), 5.08(\mathrm{t}, J=7.9 \mathrm{~Hz}, 1 \mathrm{H}), 4.85(\mathrm{~d}, J=14.9 \mathrm{~Hz}, 1 \mathrm{H}), 4.35(\mathrm{~d}, J=15.0 \mathrm{~Hz}, 1 \mathrm{H})$, $3.81(\mathrm{~d}, J=10.0 \mathrm{~Hz}, 1 \mathrm{H}), 3.60(\mathrm{dd}, J=17.4,8.0 \mathrm{~Hz}, 1 \mathrm{H}), 3.52(\mathrm{~d}, J=18.2 \mathrm{~Hz}, 1 \mathrm{H})$, 3.37 (d, $J=18.1 \mathrm{~Hz}, 1 \mathrm{H}), 3.07$ (t, $J=11.0 \mathrm{~Hz}, 2 \mathrm{H}), 2.45$ (s, 3H), 2.15 (s, 3H).

${ }^{13} \mathrm{C}$ NMR $\left(100 \mathrm{MHz}, \mathrm{CDCl}_{3}\right) \delta 170.1,164.8,151.9,146.4,144.1,138.3,138.2,137.0$, $132.9,129.3,128.8,128.8,128.7,128.0,127.6,127.5,123.1,111.2,77.9,52.8,47.1$, $46.5,38.7,14.5,13.8$.

HRMS (ESI) $m / z$ calculated for $\mathrm{C}_{33} \mathrm{H}_{31} \mathrm{ClN}_{3} \mathrm{O}_{3}{ }^{+}[\mathrm{M}+\mathrm{H}]^{+}:$552.2049, found 552.2045.

HPLC analysis: (IC column, Hexane:2-propanol $=40: 60$, flow rate $=0.5 \mathrm{~mL} / \mathrm{min}$, wavelength $=254 \mathrm{~nm}$ ): $R \mathrm{t}=53.61$ (major), 46.89 (minor).

(2S,3S,4S)-6-benzyl-4-(4-bromophenyl)-2-(2-(3,5-dimethyl-1H-pyrazol-1-yl)-2-ox oethyl)-3-phenyl-3,4,5,6-tetrahydropyrano[2,3-c]pyrrol-7(2H)-one (3ha) 
<smiles>Cc1cc(C)n(C(=O)CC2OC3=C(CN(Cc4ccccc4)C3=O)[C@@H](c3ccc(Br)cc3)[C@H]2c2ccccc2)n1</smiles>

White solid, $117.4 \mathrm{mg}, 98 \%$ yield, $\mathrm{mp}=157.8-158.7^{\circ} \mathrm{C},[\alpha] \mathrm{D}^{25}=-55.1(\mathrm{c} 1.098$, $\mathrm{CH}_{2} \mathrm{Cl}_{2}$ ), $98: 2 \mathrm{dr},>99 \%$ ee.

${ }^{1} \mathrm{H}$ NMR (400 MHz, $\left.\mathrm{CDCl}_{3}\right) \delta 7.22(\mathrm{~d}, J=7.4 \mathrm{~Hz}, 2 \mathrm{H}), 7.18(\mathrm{dd}, J=7.5,3.1 \mathrm{~Hz}, 3 \mathrm{H})$, $7.10(\mathrm{dd}, J=16.3,7.2 \mathrm{~Hz}, 5 \mathrm{H}), 6.86(\mathrm{~d}, J=6.5 \mathrm{~Hz}, 2 \mathrm{H}), 6.62(\mathrm{~d}, J=7.9 \mathrm{~Hz}, 2 \mathrm{H})$, $5.80(\mathrm{~s}, 1 \mathrm{H}), 4.99(\mathrm{t}, J=10.6 \mathrm{~Hz}, 1 \mathrm{H}), 4.77(\mathrm{~d}, J=14.9 \mathrm{~Hz}, 1 \mathrm{H}), 4.27(\mathrm{~d}, J=14.9 \mathrm{~Hz}$, $1 \mathrm{H}), 3.72(\mathrm{~d}, J=10.0 \mathrm{~Hz}, 1 \mathrm{H}), 3.52(\mathrm{dd}, J=17.4,8.0 \mathrm{~Hz}, 1 \mathrm{H}), 3.43(\mathrm{~d}, J=18.3 \mathrm{~Hz}$, $1 \mathrm{H}), 3.29(\mathrm{~d}, J=18.1 \mathrm{~Hz}, 1 \mathrm{H}), 3.04-2.96(\mathrm{~m}, 2 \mathrm{H}), 2.37$ (s, 3H), 2.07 (s, 3H).

${ }^{13} \mathrm{C}$ NMR $\left(100 \mathrm{MHz}, \mathrm{CDCl}_{3}\right) \delta 170.1,164.8,151.9,146.4,144.1,138.8,138.3,137.0$, $131.7,129.7,128.8,128.8,128.0,127.6,127.5,123.0,121.1,111.2,77.9,52.7,47.2$, $47.1,46.5,38.7,14.5,13.8$.

HRMS (ESI) $m / z$ calculated for $\mathrm{C}_{33} \mathrm{H}_{31} \mathrm{BrN}_{3} \mathrm{O}_{3}{ }^{+}[\mathrm{M}+\mathrm{H}]^{+}:$596.1543, found 596.1543.

HPLC analysis: (IC column, Hexane:2-propanol $=40: 60$, flow rate $=0.5 \mathrm{~mL} / \mathrm{min}$, wavelength $=254 \mathrm{~nm}$ ): $R \mathrm{t}=56.50$ (major), 50.09(minor).

(2S,3S,4S)-6-benzyl-2-(2-(3,5-dimethyl-1H-pyrazol-1-yl)-2-oxoethyl)-4-(4-methox yphenyl)-3-phenyl-3,4,5,6-tetrahydropyrano[2,3-c]pyrrol-7(2H)-one (3ia)<smiles>COc1ccc(C2C3=C(OC2C(=O)n2nc(C)cc2C)C(c2ccccc2)C3c2ccccc2)cc1</smiles>

White solid, $107.1 \mathrm{mg}, 98 \%$ yield, $\mathrm{mp}=112.3-112.7{ }^{\circ} \mathrm{C},[\alpha] \mathrm{D}^{25}=-56.3($ c 1.021 , $\left.\mathrm{CH}_{2} \mathrm{Cl}_{2}\right), 96: 4 \mathrm{dr},>99 \%$ ee.

${ }^{1} \mathrm{H}$ NMR (400 MHz, $\left.\mathrm{CDCl}_{3}\right) \delta 7.34-7.27(\mathrm{~m}, 3 \mathrm{H}), 7.21$ (d, J= $\left.7.4 \mathrm{~Hz}, 2 \mathrm{H}\right), 7.16$ (d, $J=6.9 \mathrm{~Hz}, 3 \mathrm{H}), 6.97(\mathrm{~s}, 2 \mathrm{H}), 6.75(\mathrm{~d}, J=6.9 \mathrm{~Hz}, 2 \mathrm{H}), 6.68(\mathrm{~d}, J=7.2 \mathrm{~Hz}, 2 \mathrm{H}), 5.90$ (s, 1H), 5.10 (t, $J=7.6 \mathrm{~Hz}, 1 \mathrm{H}), 4.87$ (d, $J=14.9 \mathrm{~Hz}, 1 \mathrm{H}), 4.35$ (d, $J=14.9 \mathrm{~Hz}, 1 \mathrm{H})$, $3.79(\mathrm{~d}, J=9.6 \mathrm{~Hz}, 1 \mathrm{H}), 3.71(\mathrm{~s}, 3 \mathrm{H}), 3.63(\mathrm{dd}, J=17.4,7.9 \mathrm{~Hz}, 1 \mathrm{H}), 3.53(\mathrm{~d}, J=$ $18.1 \mathrm{~Hz}, 1 \mathrm{H}), 3.41$ (d, $J=18.1 \mathrm{~Hz}, 1 \mathrm{H}), 3.14-3.05(\mathrm{~m}, 2 \mathrm{H}), 2.48$ (s, 3H), 2.17 (s, $3 \mathrm{H})$. 
${ }^{13} \mathrm{C} \mathrm{NMR}\left(100 \mathrm{MHz}, \mathrm{CDCl}_{3}\right) \delta 170.3,165.0,158.5,151.8,146.0,144.1,138.9,137.1$, $131.5,129.0,128.7,128.7,128.0,127.6,127.3,124.4,113.9,111.2,77.9,55.1,52.9$, $47.3,46.9,46.5,38.8,14.5,13.8$.

HRMS (ESI) $m / z$ calculated for $\mathrm{C}_{34} \mathrm{H}_{34} \mathrm{~N}_{3} \mathrm{O}_{4}{ }^{+}[\mathrm{M}+\mathrm{H}]^{+}: 548.2544$, found 548.2540.

HPLC analysis: (IA column, Hexane:2-propanol $=80: 20$, flow rate $=0.4 \mathrm{~mL} / \mathrm{min}$, wavelength $=254 \mathrm{~nm}$ ): $R \mathrm{t}=30.30$ (major), 27.42 (minor).

(2S,3S,4S)-6-benzyl-2-(2-(3,5-dimethyl-1H-pyrazol-1-yl)-2-oxoethyl)-3-phenyl-4-( p-tolyl)-3,4,5,6-tetrahydropyrano[2,3-c]pyrrol-7(2H)-one (3ja)<smiles>Cc1ccc(C2C3=C(OC2C(=O)CC2CN(Cc4ccccc4)C(=O)C2c2ccccc2)C(c2ccccc2)C3)c(C)c1</smiles>

White solid, $104.2 \mathrm{mg}, 98 \%$ yield, $\mathrm{mp}=122.0-122.8{ }^{\circ} \mathrm{C},[\alpha] \mathrm{D}^{25}=-57.8(\mathrm{c} 1.067$, $\left.\mathrm{CH}_{2} \mathrm{Cl}_{2}\right), 98: 2 \mathrm{dr},>99 \%$ ee.

${ }^{1} \mathrm{H}$ NMR (400 MHz, $\left.\mathrm{CDCl}_{3}\right) \delta 7.32-7.27$ (m, 2H), 7.23 (d, $\left.J=6.9 \mathrm{~Hz}, 1 \mathrm{H}\right), 7.19$ (d, $J=7.5 \mathrm{~Hz}, 2 \mathrm{H}), 7.13(\mathrm{t}, J=8.4 \mathrm{~Hz}, 3 \mathrm{H}), 6.98-6.90(\mathrm{~m}, 4 \mathrm{H}), 6.70(\mathrm{~d}, J=7.2 \mathrm{~Hz}, 2 \mathrm{H})$, $5.88(\mathrm{~s}, 1 \mathrm{H}), 5.08(\mathrm{t}, J=9.2 \mathrm{~Hz}, 1 \mathrm{H}), 4.86(\mathrm{~d}, J=15.0 \mathrm{~Hz}, 1 \mathrm{H}), 4.32(\mathrm{~d}, J=15.0 \mathrm{~Hz}$, $1 \mathrm{H}), 3.79$ (d, $J=10.0 \mathrm{~Hz}, 1 \mathrm{H}), 3.61(\mathrm{dd}, J=17.3,8.1 \mathrm{~Hz}, 1 \mathrm{H}), 3.51(\mathrm{~d}, J=18.1 \mathrm{~Hz}$, 1H), 3.39 (d, $J=18.2 \mathrm{~Hz}, 1 \mathrm{H}), 3.08$ (q, $J=7.9 \mathrm{~Hz}, 2 \mathrm{H}), 2.46$ (s, 3H), 2.22 (s, 3H), $2.16(\mathrm{~s}, 3 \mathrm{H})$.

${ }^{13} \mathrm{C}$ NMR $\left(100 \mathrm{MHz}, \mathrm{CDCl}_{3}\right) \delta 170.3,165.1,151.9,146.0,144.2,138.8,137.1,136.7$, $136.5,129.2,128.7,128.7,128.0,127.9,127.6,127.3,124.3,111.2,78.0,52.8,47.4$, $47.3,46.6,38.9,21.0,14.5,13.8$.

HRMS (ESI) $m / z$ calculated for $\mathrm{C}_{34} \mathrm{H}_{34} \mathrm{~N}_{3} \mathrm{O}_{3}{ }^{+}[\mathrm{M}+\mathrm{H}]^{+}:$532.2595, found 532.2594.

HPLC analysis: (IA column, Hexane:2-propanol $=80: 20$, flow rate $=0.4 \mathrm{~mL} / \mathrm{min}$, wavelength $=254 \mathrm{~nm}$ ): $R \mathrm{t}=23.76$ (major), 21.86 (minor).

(2S,3S,4S)-6-benzyl-4-(3,5-dichlorophenyl)-2-(2-(3,5-dimethyl-1H-pyrazol-1-yl)-2 -oxoethyl)-3-phenyl-3,4,5,6-tetrahydropyrano[2,3-c]pyrrol-7(2H)-one (3ka)<smiles>Cc1cc(C)n(C(=O)C[C@@H]2CC3=C(C(=O)O2)[C@@H](c2cc(Cl)cc(Cl)c2)[C@H]3c2ccccc2)n1</smiles> 
White solid, $111.6 \mathrm{mg}, 95 \%$ yield, $\mathrm{mp}=165.9-166.7{ }^{\circ} \mathrm{C},[\alpha] \mathrm{D}^{25}=-53.1(\mathrm{c}$ 1.061, $\mathrm{CH}_{2} \mathrm{Cl}_{2}$ ), 94:6 dr, 99\% ee.

${ }^{1} \mathrm{H}$ NMR $\left(400 \mathrm{MHz}, \mathrm{CDCl}_{3}\right) \delta 7.34-7.25(\mathrm{~m}, 3 \mathrm{H}), 7.19(\mathrm{dd}, J=11.5,5.1 \mathrm{~Hz}, 5 \mathrm{H})$, $7.12(\mathrm{~s}, 1 \mathrm{H}), 6.97(\mathrm{~d}, J=6.6 \mathrm{~Hz}, 2 \mathrm{H}), 6.72(\mathrm{~s}, 2 \mathrm{H}), 5.88(\mathrm{~s}, 1 \mathrm{H}), 5.05$ (t, $J=9.3 \mathrm{~Hz}$, $1 \mathrm{H}), 4.88(\mathrm{~d}, J=15.0 \mathrm{~Hz}, 1 \mathrm{H}), 4.37(\mathrm{~d}, J=15.0 \mathrm{~Hz}, 1 \mathrm{H}), 3.80(\mathrm{~d}, J=10.1 \mathrm{~Hz}, 1 \mathrm{H})$, $3.65-3.49$ (m, 2H), $3.38(\mathrm{~d}, J=18.1 \mathrm{~Hz}, 1 \mathrm{H}), 3.15-3.05(\mathrm{~m}, 2 \mathrm{H}), 2.44$ (s, 3H), 2.15 $(\mathrm{s}, 3 \mathrm{H})$.

${ }^{13} \mathrm{C}$ NMR $\left(100 \mathrm{MHz}, \mathrm{CDCl}_{3}\right) \delta 170.0,164.5,151.9,146.7,144.1,143.3,137.8,136.9$, $135.1,129.0,128.8,128.0,127.8,127.7,127.6,126.5,122.0,111.3,77.9,52.4,47.2$, $46.9,46.5,38.6,14.5,13.8$.

HRMS (ESI) $\mathrm{m} / \mathrm{z}$ calculated for $\mathrm{C}_{33} \mathrm{H}_{30} \mathrm{Cl}_{2} \mathrm{~N}_{3} \mathrm{O}_{3}{ }^{+}[\mathrm{M}+\mathrm{H}]^{+}: 586.1659$, found 586.1653 .

HPLC analysis: (IC column, Hexane:2-propanol $=40: 60$, flow rate $=0.5 \mathrm{~mL} / \mathrm{min}$, wavelength $=254 \mathrm{~nm}$ ): $R \mathrm{t}=73.15$ (major), 42.71 (minor).

(2S,3S,4S)-6-benzyl-2-(2-(3,5-dimethyl-1H-pyrazol-1-yl)-2-oxoethyl)-4-(naphthal en-2-yl)-3-phenyl-3,4,5,6-tetrahydropyrano[2,3-c]pyrrol-7(2H)-one (3la)

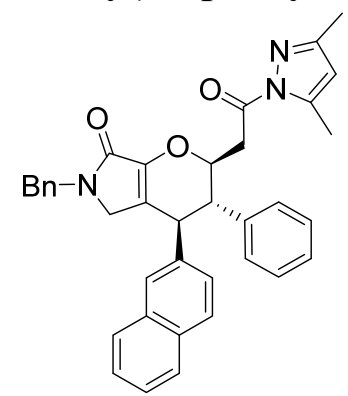

White solid, $110.9 \mathrm{mg}, 98 \%$ yield, $\mathrm{mp}=137.1-138.6{ }^{\circ} \mathrm{C},[\alpha] \mathrm{D}^{25}=-69.8$ (c 1.134, $\mathrm{CH}_{2} \mathrm{Cl}_{2}$ ), $97: 3 \mathrm{dr},>99 \%$ ee.

${ }^{1} \mathrm{H}$ NMR (400 MHz, $\left.\mathrm{CDCl}_{3}\right) \delta 7.77-7.58(\mathrm{~m}, 3 \mathrm{H}), 7.40$ (d, J=3.5 Hz, 2H), $7.31-$ $7.23(\mathrm{~m}, 4 \mathrm{H}), 7.19$ (d, J=7.0 Hz, 2H), $7.15-7.02(\mathrm{~m}, 4 \mathrm{H}), 6.98(\mathrm{~s}, 2 \mathrm{H}), 5.89(\mathrm{~s}, 1 \mathrm{H})$, $5.17(\mathrm{t}, J=7.8 \mathrm{~Hz}, 1 \mathrm{H}), 4.87(\mathrm{~d}, J=14.9 \mathrm{~Hz}, 1 \mathrm{H}), 4.29(\mathrm{~d}, J=14.9 \mathrm{~Hz}, 1 \mathrm{H}), 4.00(\mathrm{~d}$, $J=10.0 \mathrm{~Hz}, 1 \mathrm{H}), 3.70(\mathrm{dd}, J=17.4,8.1 \mathrm{~Hz}, 1 \mathrm{H}), 3.51(\mathrm{~d}, J=18.2 \mathrm{~Hz}, 1 \mathrm{H}), 3.41-$ $3.26(\mathrm{~m}, 2 \mathrm{H}), 3.15(\mathrm{dd}, J=17.4,3.0 \mathrm{~Hz}, 1 \mathrm{H}), 2.49(\mathrm{~s}, 3 \mathrm{H}), 2.18(\mathrm{~s}, 3 \mathrm{H})$.

${ }^{13} \mathrm{C}$ NMR (100 MHz, Chloroform-d) $\delta 170.2,151.8,146.2,144.1,138.7,137.1,128.8$, $128.7,128.5,128.0,127.6,127.6,127.4,127.3,126.2,125.9,125.5,111.3,78.0,52.5$, $47.73,47.3,46.5,38.8,14.5,13.8$.

HRMS (ESI) $m / z$ calculated for $\mathrm{C}_{37} \mathrm{H}_{34} \mathrm{~N}_{3} \mathrm{O}_{3}{ }^{+}[\mathrm{M}+\mathrm{H}]^{+}:$568.2595, found 568.2592.

HPLC analysis: (IC column, Hexane:2-propanol $=40: 60$, flow rate $=0.5 \mathrm{~mL} / \mathrm{min}$, wavelength $=254 \mathrm{~nm}$ ): $R \mathrm{t}=63.03$ (major), 49.37 (minor). 
(2S,3S,4R)-6-benzyl-2-(2-(3,5-dimethyl-1H-pyrazol-1-yl)-2-oxoethyl)-3-phenyl-4-( thiophen-2-yl)-3,4,5,6-tetrahydropyrano[2,3-c]pyrrol-7(2H)-one (3ma)<smiles>Cc1cc(C)n(C(=O)CC2OC3=C(CN(Cc4ccccc4)C3=O)C(c3cccs3)C2c2ccccc2)n1</smiles>

Yellow solid, $99.3 \mathrm{mg}, 95 \%$ yield, $\mathrm{mp}=150.9-151.4{ }^{\circ} \mathrm{C},[\alpha] \mathrm{D}^{25}=-28.9($ c 0.976 , $\left.\mathrm{CH}_{2} \mathrm{Cl}_{2}\right), 98: 2 \mathrm{dr},>99 \%$ ee.

${ }^{1} \mathrm{H}$ NMR (400 MHz, $\left.\mathrm{CDCl}_{3}\right) \delta 7.30(\mathrm{~d}, J=6.9 \mathrm{~Hz}, 2 \mathrm{H}), 7.25(\mathrm{~s}, 1 \mathrm{H}), 7.19(\mathrm{dd}, J=$ 16.1, 7.0 Hz, 5H), $7.04(\mathrm{~d}, J=7.6 \mathrm{~Hz}, 3 \mathrm{H}), 6.75-6.70(\mathrm{~m}, 1 \mathrm{H}), 6.50(\mathrm{~d}, J=2.5 \mathrm{~Hz}$, $1 \mathrm{H}), 5.89(\mathrm{~s}, 1 \mathrm{H}), 5.09$ (t, $J=8.0 \mathrm{~Hz}, 1 \mathrm{H}), 4.85(\mathrm{~d}, J=15.0 \mathrm{~Hz}, 1 \mathrm{H}), 4.36$ (d, $J=15.0$ $\mathrm{Hz}, 1 \mathrm{H}), 4.21(\mathrm{~d}, J=10.1 \mathrm{~Hz}, 1 \mathrm{H}), 3.70-3.49(\mathrm{~m}, 3 \mathrm{H}), 3.21(\mathrm{t}, J=10.4 \mathrm{~Hz}, 1 \mathrm{H})$, $3.08(\mathrm{~d}, J=17.4 \mathrm{~Hz}, 1 \mathrm{H}), 2.46(\mathrm{~s}, 3 \mathrm{H}), 2.17$ (s, 3H).

${ }^{13} \mathrm{C} \mathrm{NMR}\left(100 \mathrm{MHz}, \mathrm{CDCl}_{3}\right) \delta 170.1,164.8,151.9,145.4,144.1,142.4,138.6,137.1$, $128.8,128.7,128.0,127.6,127.6,126.6,125.9,124.4,123.3,111.2,78.1,53.1,47.3$, $46.4,42.8,38.8,14.5,13.8$.

HRMS (ESI) $m / z$ calculated for $\mathrm{C}_{31} \mathrm{H}_{30} \mathrm{~N}_{3} \mathrm{O}_{3} \mathrm{~S}^{+}[\mathrm{M}+\mathrm{H}]^{+}:$: 524.2002, found 524.1999.

HPLC analysis: (IA column, Hexane:2-propanol $=80: 20$, flow rate $=0.4 \mathrm{~mL} / \mathrm{min}$, wavelength $=254 \mathrm{~nm}$ ): $R \mathrm{t}=30.69$ (major), 26.86 (minor).

(2S,3S,4R)-6-benzyl-2-(2-(3,5-dimethyl-1H-pyrazol-1-yl)-2-oxoethyl)-3-phenyl-4-( (E)-styryl)-3,4,5,6-tetrahydropyrano[2,3-c]pyrrol-7(2H)-one (3na)<smiles>Cc1cc(C)n(C(=O)CC2OC3=C(CN(Cc4ccccc4)C3=O)[C@@H](/C=C/c3ccccc3)[C@H]2c2ccccc2)n1</smiles>

Yellow solid, $93.6 \mathrm{mg}, 86 \%$ yield, $\mathrm{mp}=97.0-97.8{ }^{\circ} \mathrm{C},[\alpha] \mathrm{D}^{25}=-44.1$ (c 0.795 , $\left.\mathrm{CH}_{2} \mathrm{Cl}_{2}\right), 92: 8 \mathrm{dr},>99 \%$ ee.

${ }^{1} \mathrm{H}$ NMR (400 MHz, $\left.\mathrm{CDCl}_{3}\right) \delta 7.34-7.28(\mathrm{~m}, 3 \mathrm{H}), 7.25-7.19(\mathrm{~m}, 6 \mathrm{H}), 7.14(\mathrm{t}, J=$ $6.7 \mathrm{~Hz}, 6 \mathrm{H}), 6.07(\mathrm{~d}, J=15.7 \mathrm{~Hz}, 1 \mathrm{H}), 5.87(\mathrm{~s}, 1 \mathrm{H}), 5.82(\mathrm{dd}, J=15.7,9.1 \mathrm{~Hz}, 1 \mathrm{H})$, $4.96(\mathrm{t}, J=7.7 \mathrm{~Hz}, 1 \mathrm{H}), 4.77(\mathrm{~d}, J=14.9 \mathrm{~Hz}, 1 \mathrm{H}), 4.46$ (d, $J=15.0 \mathrm{~Hz}, 1 \mathrm{H}), 3.66$ (s, 2H), 3.57 (dd, $J=17.5,7.9 \mathrm{~Hz}, 1 \mathrm{H}), 3.46(\mathrm{t}, J=9.5 \mathrm{~Hz}, 1 \mathrm{H}), 3.12$ (dd, $J=17.5,3.1$ $\mathrm{Hz}, 1 \mathrm{H}), 3.01(\mathrm{t}, J=10.2 \mathrm{~Hz}, 1 \mathrm{H}), 2.45(\mathrm{~s}, 3 \mathrm{H}), 2.16(\mathrm{~s}, 3 \mathrm{H})$. 
${ }^{13} \mathrm{C} \mathrm{NMR}\left(100 \mathrm{MHz}, \mathrm{CDCl}_{3}\right) \delta 170.3,165.0,151.9,145.2,144.2,139.0,137.1,136.3$, $133.7,128.9,128.8,128.5,128.2,127.8,127.7,127.6,127.5,126.2,123.2,111.2$, $77.7,50.1,47.6,46.6,44.9,38.7,14.6,13.8$.

HRMS (ESI) $m / z$ calculated for $\mathrm{C}_{35} \mathrm{H}_{34} \mathrm{~N}_{3} \mathrm{O}_{3}{ }^{+}[\mathrm{M}+\mathrm{H}]^{+}:$544.2595, found 544.2593.

HPLC analysis: (IA column, Hexane:EtOH $=80: 20$, flow rate $=0.3 \mathrm{~mL} / \mathrm{min}$, wavelength $=254 \mathrm{~nm}$ ): $R \mathrm{t}=51.68$ (major), 45.96 (minor).

(2S,3S,4R)-Methyl-(2-(3,5-dimethyl-1H-pyrazol-1-yl)-2-oxoethyl)-3,4-diphenyl-3, 4-dihydr-o-2H-pyran-6-carboxylate (3ta)<smiles>COC(=O)C1=C[C@H](c2ccccc2)[C@@H](CC(=O)n2nc(C)cc2C)OC1c1ccccc1</smiles>

White solid, $68.1 \mathrm{mg}, 79 \%$ yield, $\mathrm{mp}=177.7-178.7{ }^{\circ} \mathrm{C},[\alpha] \mathrm{D}^{25}=81.3($ c 0.697 , $\mathrm{CH}_{2} \mathrm{Cl}_{2}$ ), $70: 30 \mathrm{dr}, 86 \%$ ee.

${ }^{1} \mathrm{H}$ NMR (400 MHz, $\left.\mathrm{CDCl}_{3}\right) \delta 7.21-7.11(\mathrm{~m}, 6 \mathrm{H}), 7.00-6.94(\mathrm{~m}, 2 \mathrm{H}), 6.89-6.84$ $(\mathrm{m}, 2 \mathrm{H}), 6.25(\mathrm{~d}, \mathrm{~J}=2.5 \mathrm{~Hz}, 1 \mathrm{H}), 5.89(\mathrm{~d}, \mathrm{~J}=1.1 \mathrm{~Hz}, 1 \mathrm{H}), 4.96(\mathrm{~m}, \mathrm{~J}=10.5,8.6,3.1$ $\mathrm{Hz}, 1 \mathrm{H}), 3.82-3.78(\mathrm{~m}, 3 \mathrm{H}), 3.77(\mathrm{~d}, \mathrm{~J}=2.6 \mathrm{~Hz}, 1 \mathrm{H}), 3.50(\mathrm{~m}, 1 \mathrm{H}), 3.07(\mathrm{~m}, 1 \mathrm{H})$, $2.89(\mathrm{t}, \mathrm{J}=10.5 \mathrm{~Hz}, 1 \mathrm{H}), 2.47(\mathrm{~d}, \mathrm{~J}=1.0 \mathrm{~Hz}, 3 \mathrm{H}), 2.17(\mathrm{~s}, 3 \mathrm{H})$.

${ }^{13} \mathrm{C}$ NMR $\left(100 \mathrm{MHz}, \mathrm{CDCl}_{3}\right) \delta 170.6,163.1,151.8,144.2,141.4,139.2,128.6,128.4$, 128.3, 127.9, 127.2, 126.9, 115.4, 111.1, 77.0, 52.2, 51.8, 47.5, 39.1, 14.44, 13.8 .

HRMS (ESI) $m / z$ calculated for $\mathrm{C}_{26} \mathrm{H}_{27} \mathrm{~N}_{2} \mathrm{O}_{4}{ }^{+}[\mathrm{M}+\mathrm{H}]^{+}: 431.1926$, found 431.1975.

HPLC analysis: (AD-H column, Hexane:2-propanol $=90: 10$, flow rate $=0.3 \mathrm{~mL} / \mathrm{min}$, wavelength $=254 \mathrm{~nm}$ ): $R \mathrm{t}=20.54$ (major), 18.13 (minor).

(2S,3S,4S)-6-benzyl-2-(2-(3,5-dimethyl-1H-pyrazol-1-yl)-2-oxoethyl)-4-phenyl-3-( o-tolyl)-3,4,5,6-tetrahydropyrano[2,3-c]pyrrol-7(2H)-one (3ab)<smiles>Cc1cc(C)n(C(=O)CC2OC3=C(CN(Cc4ccccc4)C3=O)C(c3ccccc3)C2c2ccccc2C)n1</smiles>

White solid, $101.3 \mathrm{mg}, 95 \%$ yield, $\mathrm{mp}=187.5-188.7{ }^{\circ} \mathrm{C},[\alpha] \mathrm{D}^{25}=-47.2(\mathrm{c} 1.001$, $\left.\mathrm{CH}_{2} \mathrm{Cl}_{2}\right), 97: 3 \mathrm{dr},>99 \%$ ee. 
${ }^{1} \mathrm{H}$ NMR $\left(400 \mathrm{MHz}, \mathrm{CDCl}_{3}\right) \delta 7.33-7.28(\mathrm{~m}, 2 \mathrm{H}), 7.23(\mathrm{~d}, J=7.4 \mathrm{~Hz}, 2 \mathrm{H}), 7.22-$ $7.16(\mathrm{~m}, 3 \mathrm{H}), 7.12(\mathrm{~s}, 3 \mathrm{H}), 7.00(\mathrm{t}, J=6.4 \mathrm{~Hz}, 1 \mathrm{H}), 6.82(\mathrm{dd}, J=16.2,6.0 \mathrm{~Hz}, 3 \mathrm{H})$, $5.86(\mathrm{~s}, 1 \mathrm{H}), 5.09(\mathrm{t}, J=7.3 \mathrm{~Hz}, 1 \mathrm{H}), 4.86(\mathrm{~d}, J=14.9 \mathrm{~Hz}, 1 \mathrm{H}), 4.39(\mathrm{~d}, J=14.9 \mathrm{~Hz}$, $1 \mathrm{H}), 3.80(\mathrm{~d}, J=9.8 \mathrm{~Hz}, 1 \mathrm{H}), 3.62(\mathrm{dd}, J=17.0,7.9 \mathrm{~Hz}, 1 \mathrm{H}), 3.53(\mathrm{~s}, 2 \mathrm{H}), 3.44(\mathrm{t}, J=$ $10.2 \mathrm{~Hz}, 1 \mathrm{H}), 3.06$ (dd, $J=17.0,3.1 \mathrm{~Hz}, 1 \mathrm{H}), 2.43$ (s, 3H), 2.15 (s, 3H), 1.70 (s, 3H).

${ }^{13} \mathrm{C}$ NMR $\left(100 \mathrm{MHz}, \mathrm{CDCl}_{3}\right) \delta 170.2,165.0,151.7,146.6,144.0,139.6,137.6,137.4$, $137.1,130.2,128.7,128.4,128.1,128.0,127.6,127.2,126.9,126.6,126.4,124.1$, $111.2,78.6,48.1,47.4,47.1,46.5,38.3,19.6,14.5,13.7$.

HRMS (ESI) $m / z$ calculated for $\mathrm{C}_{34} \mathrm{H}_{34} \mathrm{~N}_{3} \mathrm{O}_{3}{ }^{+}[\mathrm{M}+\mathrm{H}]^{+}:$532.2595, found 532.2593.

HPLC analysis: (IA column, Hexane:2-propanol $=90: 10$, flow rate $=0.4 \mathrm{~mL} / \mathrm{min}$, wavelength $=254 \mathrm{~nm}$ ): $R \mathrm{t}=48.95$ (major), 43.47 (minor).

(2S,3S,4S)-6-benzyl-2-(2-(3,5-dimethyl-1H-pyrazol-1-yl)-2-oxoethyl)-3-(3-methox yphenyl)-4-phenyl-3,4,5,6-tetrahydropyrano[2,3-c]pyrrol-7(2H)-one (3ac)<smiles>COc1cccc(C2C(=C(c3ccccc3)c3ccccc3)C(=O)OC2CC(=O)n2nc(C)cc2C)c1</smiles>

White solid, $102.6 \mathrm{mg}, 94 \%$ yield, $\mathrm{mp}=97.7-98.7{ }^{\circ} \mathrm{C},[\alpha] \mathrm{D}^{25}=-52.0$ (c 1.030, $\mathrm{CH}_{2} \mathrm{Cl}_{2}$ ), 95:5 dr, $>99 \%$ ee.

${ }^{1} \mathrm{H}$ NMR (400 MHz, $\left.\mathrm{CDCl}_{3}\right) \delta 7.33-7.24(\mathrm{~m}, 3 \mathrm{H}), 7.19(\mathrm{~d}, J=7.1 \mathrm{~Hz}, 2 \mathrm{H}), 7.13$ (s, $3 \mathrm{H}), 7.10-7.04(\mathrm{~m}, 1 \mathrm{H}), 6.88-6.79(\mathrm{~m}, 2 \mathrm{H}), 6.65(\mathrm{~d}, J=7.8 \mathrm{~Hz}, 1 \mathrm{H}), 6.55(\mathrm{~d}, J=$ $6.6 \mathrm{~Hz}, 1 \mathrm{H}), 6.45(\mathrm{~s}, 1 \mathrm{H}), 5.88(\mathrm{~s}, 1 \mathrm{H}), 5.07$ (t, $J=7.9 \mathrm{~Hz}, 1 \mathrm{H}), 4.87$ (d, J=14.9 Hz, $1 \mathrm{H}), 4.32(\mathrm{~d}, J=15.0 \mathrm{~Hz}, 1 \mathrm{H}), 3.81(\mathrm{~d}, J=9.9 \mathrm{~Hz}, 1 \mathrm{H}), 3.64(\mathrm{~s}, 3 \mathrm{H}), 3.59-3.47(\mathrm{~m}$, 2H), $3.39(\mathrm{~d}, J=18.1 \mathrm{~Hz}, 1 \mathrm{H}), 3.17-3.04(\mathrm{~m}, 2 \mathrm{H}), 2.47$ (s, 3H), $2.16(\mathrm{~s}, 3 \mathrm{H})$.

${ }^{13} \mathrm{C}$ NMR $\left(100 \mathrm{MHz}, \mathrm{CDCl}_{3}\right) \delta 170.3,165.0,159.6,151.8,146.1,144.2,140.2,139.7$, $137.1,129.7,128.7,128.7,128.6,128.0,127.6,127.2,124.0,120.6,114.3,112.6$, $111.2,77.9,55.1,52.9,47.6,47.3,46.5,38.8,14.5,13.8$.

HRMS (ESI) $m / z$ calculated for $\mathrm{C}_{34} \mathrm{H}_{34} \mathrm{~N}_{3} \mathrm{O}_{4}{ }^{+}[\mathrm{M}+\mathrm{H}]^{+}:$548.2544, found 548.2542.

HPLC analysis: (IC column, Hexane:2-propanol $=40: 60$, flow rate $=0.5 \mathrm{~mL} / \mathrm{min}$, wavelength $=254 \mathrm{~nm}$ ): $R \mathrm{t}=72.11$ (major), 57.48 (minor).

(2S,3S,4S)-6-benzyl-3-(3-bromophenyl)-2-(2-(3,5-dimethyl-1H-pyrazol-1-yl)-2-ox oethyl)-4-phenyl-3,4,5,6-tetrahydropyrano[2,3-c]pyrrol-7(2H)-one (3ad) 


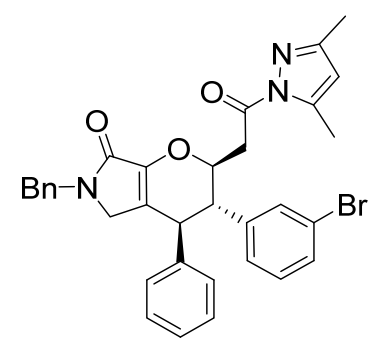

White solid, $115.0 \mathrm{mg}, 96 \%$ yield, $\mathrm{mp}=95.6-97.4{ }^{\circ} \mathrm{C},[\alpha] \mathrm{D}^{25}=-67.1$ (c 1.122, $\left.\mathrm{CH}_{2} \mathrm{Cl}_{2}\right), 94: 6 \mathrm{dr},>99 \%$ ee.

${ }^{1} \mathrm{H}$ NMR (400 MHz, $\left.\mathrm{CDCl}_{3}\right) \delta 7.28(\mathrm{~s}, 2 \mathrm{H}), 7.23(\mathrm{~d}, J=7.0 \mathrm{~Hz}, 2 \mathrm{H}), 7.18(\mathrm{~d}, J=6.6$ $\mathrm{Hz}, 2 \mathrm{H}), 7.13$ (s, 4H), $7.00(\mathrm{~s}, 1 \mathrm{H}), 6.82(\mathrm{~s}, 3 \mathrm{H}), 5.88(\mathrm{~s}, 1 \mathrm{H}), 5.05(\mathrm{~s}, 1 \mathrm{H}), 4.84(\mathrm{~d}, J$ $=14.8 \mathrm{~Hz}, 1 \mathrm{H}), 4.32(\mathrm{~d}, J=14.8 \mathrm{~Hz}, 1 \mathrm{H}), 3.79(\mathrm{~d}, J=9.2 \mathrm{~Hz}, 1 \mathrm{H}), 3.51(\mathrm{~d}, J=17.5$ $\mathrm{Hz}, 2 \mathrm{H}), 3.39$ (d, $J=17.9 \mathrm{~Hz}, 1 \mathrm{H}), 3.19$ (d, $J=16.5 \mathrm{~Hz}, 1 \mathrm{H}), 3.14-3.06$ (m, 1H), $2.44(\mathrm{~s}, 3 \mathrm{H}), 2.17$ (s, 3H).

${ }^{13} \mathrm{C}$ NMR $\left(100 \mathrm{MHz}, \mathrm{CDCl}_{3}\right) \delta 169.9,164.8,152.1,146.1,144.1,141.0,139.1,137.0$, 130.6, 130.2, 128.7, 128.0, 127.6, 127.4, 123.9, 122.7, 111.4, 77.7, 52.7, 47.6, 47.2, $46.5,38.9,14.5,13.9$.

HRMS (ESI) $m / z$ calculated for $\mathrm{C}_{33} \mathrm{H}_{31} \mathrm{BrN}_{3} \mathrm{O}_{3}{ }^{+}[\mathrm{M}+\mathrm{H}]^{+}:$596.1543, found 596.1543.

HPLC analysis: (IC column, Hexane:2-propanol $=40: 60$, flow rate $=0.5 \mathrm{~mL} / \mathrm{min}$, wavelength $=254 \mathrm{~nm}$ ): $R \mathrm{t}=62.97$ (major), 54.81 (minor).

(2S,3S,4S)-6-benzyl-2-(2-(3,5-dimethyl-1H-pyrazol-1-yl)-2-oxoethyl)-3-(4-methox yphenyl)-4-phenyl-3,4,5,6-tetrahydropyrano[2,3-c]pyrrol-7(2H)-one (3ae)<smiles>COc1ccc([C@@H]2C3=C(C(=O)N(Cc4ccccc4)C3)[C@H](CC(=O)n3nc(C)cc3C)O[C@H]2c2ccccc2)cc1</smiles>

White solid, $106.2 \mathrm{mg}, 97 \%$ yield, $\mathrm{mp}=94.7-95.3{ }^{\circ} \mathrm{C},[\alpha] \mathrm{D}^{25}=-55.5($ c 1.061, $\mathrm{CH}_{2} \mathrm{Cl}_{2}$ ), $97: 3 \mathrm{dr},>99 \%$ ee.

${ }^{1} \mathrm{H}$ NMR $\left(400 \mathrm{MHz}, \mathrm{CDCl}_{3}\right) \delta 7.32-7.24(\mathrm{~m}, 3 \mathrm{H}), 7.21-7.18(\mathrm{~m}, 2 \mathrm{H}), 7.13(\mathrm{dd}, J=$ 4.9, $1.7 \mathrm{~Hz}, 3 \mathrm{H}), 6.88-6.79(\mathrm{~m}, 4 \mathrm{H}), 6.67$ (d, $J=8.4 \mathrm{~Hz}, 2 \mathrm{H}), 5.88(\mathrm{~s}, 1 \mathrm{H}), 5.03$ (t, $J$ $=7.6 \mathrm{~Hz}, 1 \mathrm{H}), 4.86(\mathrm{~d}, J=15.0 \mathrm{~Hz}, 1 \mathrm{H}), 4.33(\mathrm{~d}, J=15.0 \mathrm{~Hz}, 1 \mathrm{H}), 3.77(\mathrm{~d}, J=10.1$ $\mathrm{Hz}, 1 \mathrm{H}), 3.69$ (s, 3H), $3.62-3.47$ (m, 2H), 3.38 (d, $J=18.1 \mathrm{~Hz}, 1 \mathrm{H}), 3.13$ (dd, $J=$ $17.3,3.4 \mathrm{~Hz}, 1 \mathrm{H}), 3.05$ (t, $J=10.4 \mathrm{~Hz}, 1 \mathrm{H}), 2.45(\mathrm{~s}, 3 \mathrm{H}), 2.16(\mathrm{~s}, 3 \mathrm{H})$.

${ }^{13} \mathrm{C}$ NMR $\left(100 \mathrm{MHz}, \mathrm{CDCl}_{3}\right) \delta 170.3,165.1,158.6,151.8,146.1,144.1,139.8,137.1$, $130.5,128.7,128.5,128.1,128.0,127.6,127.1,124.1,124.1,114.0,111.2,78.2,55.1$, $52.1,47.6,47.3,46.5,38.9,14.5,13.8$.

HRMS (ESI) $m / z$ calculated for $\mathrm{C}^{34} \mathrm{H}^{34} \mathrm{~N}^{3} \mathrm{O}^{4+}[\mathrm{M}+\mathrm{H}]^{+}:$548.2544, found 548.2543. 
HPLC analysis: (IC column, Hexane:2-propanol $=40: 60$, flow rate $=0.5 \mathrm{~mL} / \mathrm{min}$, wavelength $=254 \mathrm{~nm}$ ): $R \mathrm{t}=79.60$ (major), 68.06 (minor).

(2S,3S,4S)-6-benzyl-3-(4-bromophenyl)-2-(2-(3,5-dimethyl-1H-pyrazol-1-yl)-2-ox oethyl)-4-phenyl-3,4,5,6-tetrahydropyrano[2,3-c]pyrrol-7(2H)-one (3af)<smiles>Cc1cc(C)n(C(=O)CC2OC3=C(CN(Cc4ccccc4)C3=O)C(c3ccccc3)C2c2ccc(Br)cc2)n1</smiles>

White solid, $113.1 \mathrm{mg}, 95 \%$ yield, $\mathrm{mp}=129.2-130.7{ }^{\circ} \mathrm{C},[\alpha] \mathrm{D}^{25}=-62.0(\mathrm{c} 1.132$, $\mathrm{CH}_{2} \mathrm{Cl}_{2}$ ), $95: 5 \mathrm{dr},>99 \%$ ee.

${ }^{1} \mathrm{H}$ NMR $\left(400 \mathrm{MHz}, \mathrm{CDCl}_{3}\right) \delta 7.28(\mathrm{dt}, J=13.8,7.4 \mathrm{~Hz}, 5 \mathrm{H}), 7.19(\mathrm{~d}, J=7.1 \mathrm{~Hz}, 2 \mathrm{H})$, $7.17-7.12(\mathrm{~m}, 3 \mathrm{H}), 6.87-6.77(\mathrm{~m}, 4 \mathrm{H}), 5.90(\mathrm{~s}, 1 \mathrm{H}), 5.08-4.98(\mathrm{~m}, 1 \mathrm{H}), 4.87$ (d, $J$ $=15.0 \mathrm{~Hz}, 1 \mathrm{H}), 4.33(\mathrm{~d}, J=15.0 \mathrm{~Hz}, 1 \mathrm{H}), 3.77(\mathrm{~d}, J=10.1 \mathrm{~Hz}, 1 \mathrm{H}), 3.60-3.47(\mathrm{~m}$, $2 \mathrm{H}), 3.38(\mathrm{~d}, J=18.2 \mathrm{~Hz}, 1 \mathrm{H}), 3.21-3.07$ (m, 2H), 2.44 (s, 3H), 2.17 (s, 3H).

${ }^{13} \mathrm{C}$ NMR $\left(100 \mathrm{MHz}, \mathrm{CDCl}_{3}\right) \delta 169.9,164.9,152.0,146.1,144.2,139.2,137.6,137.0$, $131.8,128.7,128.7,128.0,128.0,127.6,127.4,123.7,121.4,111.3,77.7,52.5,47.6$, $47.2,46.5,38.8,14.5,13.8$.

HRMS (ESI) $m / z$ calculated for $\mathrm{C}_{33} \mathrm{H}_{31} \mathrm{BrN}_{3} \mathrm{O}_{3}{ }^{+}[\mathrm{M}+\mathrm{H}]^{+}:$596.1543, found 596.1541.

HPLC analysis: (IC column, Hexane:2-propanol $=40: 60$, flow rate $=0.5 \mathrm{~mL} / \mathrm{min}$, wavelength $=254 \mathrm{~nm}$ ): $R \mathrm{t}=60.89$ (major), 53.04 (minor).

(2S,3S,4S)-6-benzyl-3-(4-chlorophenyl)-2-(2-(3,5-dimethyl-1H-pyrazol-1-yl)-2-ox oethyl)-4-phenyl-3,4,5,6-tetrahydropyrano[2,3-c]pyrrol-7(2H)-one (3ag)<smiles></smiles>

White solid, $106.5 \mathrm{mg}, 96 \%$ yield, $\mathrm{mp}=90.5-92.2{ }^{\circ} \mathrm{C},[\alpha] \mathrm{D}^{25}=-57.5$ (c 1.071, $\left.\mathrm{CH}_{2} \mathrm{Cl}_{2}\right), 98: 2 \mathrm{dr},>99 \%$ ee.

${ }^{1} \mathrm{H}$ NMR $\left(400 \mathrm{MHz}, \mathrm{CDCl}_{3}\right) \delta 7.33-7.26(\mathrm{~m}, 3 \mathrm{H}), 7.19(\mathrm{~d}, J=7.0 \mathrm{~Hz}, 2 \mathrm{H}), 7.17-$ $7.13(\mathrm{~m}, 3 \mathrm{H}), 7.11(\mathrm{~d}, J=8.1 \mathrm{~Hz}, 2 \mathrm{H}), 6.92-6.79(\mathrm{~m}, 4 \mathrm{H}), 5.90(\mathrm{~s}, 1 \mathrm{H}), 5.09-5.00$ $(\mathrm{m}, 1 \mathrm{H}), 4.87(\mathrm{~d}, J=15.0 \mathrm{~Hz}, 1 \mathrm{H}), 4.33(\mathrm{~d}, J=15.0 \mathrm{~Hz}, 1 \mathrm{H}), 3.77(\mathrm{~d}, J=10.1 \mathrm{~Hz}$, $1 \mathrm{H}), 3.59-3.48(\mathrm{~m}, 2 \mathrm{H}), 3.39(\mathrm{~d}, J=18.2 \mathrm{~Hz}, 1 \mathrm{H}), 3.19-3.07(\mathrm{~m}, 2 \mathrm{H}), 2.45(\mathrm{~s}, 3 \mathrm{H})$, $2.17(\mathrm{~s}, 3 \mathrm{H})$. 
${ }^{13} \mathrm{C} \mathrm{NMR}\left(100 \mathrm{MHz}, \mathrm{CDCl}_{3}\right) \delta 170.0,164.9,152.0,146.1,144.2,139.2,137.1,137.0$, $133.2,128.8,128.7,128.7,128.0,127.6,127.4,123.8,111.3,77.8,52.4,47.6,47.3$, $46.6,38.9,14.5,13.8$.

HRMS (ESI) $m / z$ calculated for $\mathrm{C}_{33} \mathrm{H}_{31} \mathrm{ClN}_{3} \mathrm{O}_{3}{ }^{+}[\mathrm{M}+\mathrm{H}]^{+}:$552.2049, found 552.2041.

HPLC analysis: (IA column, Hexane:2-propanol $=80: 20$, flow rate $=0.4 \mathrm{~mL} / \mathrm{min}$, wavelength $=254 \mathrm{~nm}$ ): $R \mathrm{t}=34.14$ (major), 27.47 (minor).

(2S,3S,4S)-6-benzyl-3-(2,4-dichlorophenyl)-2-(2-(3,5-dimethyl-1H-pyrazol-1-yl)-2 -oxoethyl)-4-phenyl-3,4,5,6-tetrahydropyrano[2,3-c]pyrrol-7(2H)-one (3ah)<smiles>Cc1cc(C)n(C(=O)CC2OC(=O)C3CN(Cc4ccccc4)CC3C2c2ccccc2)n1</smiles>

White solid, $110.6 \mathrm{mg}, 94 \%$ yield, $\mathrm{mp}=93.4-94.5{ }^{\circ} \mathrm{C},[\alpha] \mathrm{D}^{25}=-65.9(\mathrm{c} 1.098$, $\mathrm{CH}_{2} \mathrm{Cl}_{2}$ ), $92: 8 \mathrm{dr}, 99 \%$ ee.

${ }^{1} \mathrm{H}$ NMR (400 MHz, $\left.\mathrm{CDCl}_{3}\right) \delta 7.29(\mathrm{dd}, J=14.2,6.9 \mathrm{~Hz}, 3 \mathrm{H}), 7.20(\mathrm{~d}, J=7.0 \mathrm{~Hz}$, $4 \mathrm{H}), 7.18-7.12(\mathrm{~m}, 3 \mathrm{H}), 7.09(\mathrm{~s}, 1 \mathrm{H}), 6.92-6.81(\mathrm{~m}, 2 \mathrm{H}), 5.89(\mathrm{~s}, 1 \mathrm{H}), 5.02(\mathrm{t}, J=$ $8.5 \mathrm{~Hz}, 1 \mathrm{H}), 4.86(\mathrm{~d}, J=15.0 \mathrm{~Hz}, 1 \mathrm{H}), 4.35(\mathrm{~d}, J=15.0 \mathrm{~Hz}, 1 \mathrm{H}), 3.94-3.74(\mathrm{~m}, 2 \mathrm{H})$, $3.63(\mathrm{dd}, J=16.7,7.6 \mathrm{~Hz}, 1 \mathrm{H}), 3.56-3.37(\mathrm{~m}, 2 \mathrm{H}), 3.09(\mathrm{dd}, J=16.7,3.6 \mathrm{~Hz}, 1 \mathrm{H})$, $2.43(\mathrm{~s}, 3 \mathrm{H}), 2.17(\mathrm{~s}, 3 \mathrm{H})$.

${ }^{13} \mathrm{C}$ NMR $\left(100 \mathrm{MHz}, \mathrm{CDCl}_{3}\right) \delta 170.0,164.8,152.0,146.2,144.1,138.2,137.0,136.3$, $135.1,133.5,129.3,129.1,128.8,128.7,128.1,128.1,127.7,127.6,127.6,123.7$, $111.4,78.3,47.7,47.2,46.8,46.6,38.5,14.5,13.8$.

HRMS (ESI) $m / z$ calculated for $\mathrm{C}_{33} \mathrm{H}_{30} \mathrm{C}_{12} \mathrm{~N}_{3} \mathrm{O}_{3}{ }^{+}[\mathrm{M}+\mathrm{H}]^{+}:$586.1659, found 586.1656.

HPLC analysis: (AD-H column, Hexane:2-propanol $=70: 30$, flow rate $=0.2 \mathrm{~mL} / \mathrm{min}$, wavelength $=254 \mathrm{~nm}$ ): $R \mathrm{t}=34.13$ (major), 28.79 (minor).

(2S,3S,4S)-6-benzyl-2-(2-(3,5-dimethyl-1H-pyrazol-1-yl)-2-oxoethyl)-3-(naphthal en-2-yl)-4-phenyl-3,4,5,6-tetrahydropyrano[2,3-c]pyrrol-7(2H)-one (3ai)<smiles>Cc1cc(C)n(C(=O)CC2OC3=C(CN(Cc4ccccc4)C3=O)C(c3ccccc3)C2c2ccc3ccccc3c2)n1</smiles>

White solid, $111.4 \mathrm{mg}, 98 \%$ yield, $\mathrm{mp}=99.5-100.7{ }^{\circ} \mathrm{C},[\alpha] \mathrm{D}^{25}=-83.2(\mathrm{c} 1.105$, $\left.\mathrm{CH}_{2} \mathrm{Cl}_{2}\right), 95: 5 \mathrm{dr},>99 \%$ ee. 
${ }^{1} \mathrm{H}$ NMR (400 MHz, $\left.\mathrm{CDCl}_{3}\right) \delta 7.74-7.60(\mathrm{~m}, 3 \mathrm{H}), 7.42-7.36(\mathrm{~m}, 3 \mathrm{H}), 7.30$ (q, $J=$ $8.0 \mathrm{~Hz}, 3 \mathrm{H}), 7.21(\mathrm{~d}, J=7.0 \mathrm{~Hz}, 2 \mathrm{H}), 7.10(\mathrm{~d}, J=15.7 \mathrm{~Hz}, 4 \mathrm{H}), 6.88-6.79(\mathrm{~m}, 2 \mathrm{H})$, $5.75(\mathrm{~s}, 1 \mathrm{H}), 5.19(\mathrm{t}, J=7.4 \mathrm{~Hz}, 1 \mathrm{H}), 4.89$ (d, $J=14.9 \mathrm{~Hz}, 1 \mathrm{H}), 4.34$ (d, $J=15.0 \mathrm{~Hz}$, $1 \mathrm{H}), 3.96(\mathrm{~d}, J=10.0 \mathrm{~Hz}, 1 \mathrm{H}), 3.63-3.51(\mathrm{~m}, 2 \mathrm{H}), 3.41(\mathrm{~d}, J=18.2 \mathrm{~Hz}, 1 \mathrm{H}), 3.28$ (t, $J=10.4 \mathrm{~Hz}, 1 \mathrm{H}), 3.16(\mathrm{dd}, J=17.2,3.4 \mathrm{~Hz}, 1 \mathrm{H}), 2.33(\mathrm{~s}, 3 \mathrm{H}), 2.11$ (s, 3H).

${ }^{13} \mathrm{C}$ NMR $\left(100 \mathrm{MHz}, \mathrm{CDCl}_{3}\right) \delta 170.1,165.1,151.8,146.2,144.0,139.6,137.1,136.0$, 133.2 , 132.6, 128.8, 128.6, 128.5, 128.1, 127.8, 127.6, 127.5, 127.2, 126.1, 126.0, $124.2,111.2,78.2,53.2,47.5,47.4,46.6,39.0,14.4,13.8$.

HRMS (ESI) $m / z$ calculated for $\mathrm{C}_{37} \mathrm{H}_{34} \mathrm{~N}_{3} \mathrm{O}_{3}{ }^{+}[\mathrm{M}+\mathrm{H}]^{+}:$568.2595, found 568.2595.

HPLC analysis: (IC column, Hexane:2-propanol $=40: 60$, flow rate $=0.5 \mathrm{~mL} / \mathrm{min}$, wavelength $=254 \mathrm{~nm}$ ): $R \mathrm{t}=83.60$ (major), 72.14 (minor).

(2S,3R,4S)-6-benzyl-2-(2-(3,5-dimethyl-1H-pyrazol-1-yl)-2-oxoethyl)-3-(furan-2-y l)-4-phenyl-3,4,5,6-tetrahydropyrano[2,3-c]pyrrol-7(2H)-one (3aj)<smiles>Cc1cc(C)n(C(=O)CC2OC(=O)C3CN(Cc4ccccc4)CC3C2c2ccccc2)n1</smiles>

White solid, $96.3 \mathrm{mg}, 95 \%$ yield, $\mathrm{mp}=176.6-177.4{ }^{\circ} \mathrm{C},[\alpha] \mathrm{D}^{25}=-16.3($ c 0.941 , $\left.\mathrm{CH}_{2} \mathrm{Cl}_{2}\right), 91: 9 \mathrm{dr},>99 \%$ ee.

${ }^{1} \mathrm{H}$ NMR (400 MHz, $\left.\mathrm{CDCl}_{3}\right) \delta 7.32-7.27(\mathrm{~m}, 3 \mathrm{H}), 7.25(\mathrm{~s}, 1 \mathrm{H}), 7.22-7.16(\mathrm{~m}, 5 \mathrm{H})$, $6.93(\mathrm{~d}, J=6.5 \mathrm{~Hz}, 2 \mathrm{H}), 6.09(\mathrm{~s}, 1 \mathrm{H}), 5.92(\mathrm{~s}, 1 \mathrm{H}), 5.86(\mathrm{~d}, J=2.7 \mathrm{~Hz}, 1 \mathrm{H}), 5.03$ (t, $J$ $=10.6 \mathrm{~Hz}, 1 \mathrm{H}), 4.85(\mathrm{~d}, J=15.0 \mathrm{~Hz}, 1 \mathrm{H}), 4.35(\mathrm{~d}, J=15.0 \mathrm{~Hz}, 1 \mathrm{H}), 4.06(\mathrm{~d}, J=10.3$ $\mathrm{Hz}, 1 \mathrm{H}), 3.63$ (dd, $J=17.6,7.1 \mathrm{~Hz}, 1 \mathrm{H}), 3.53$ (d, $J=18.2 \mathrm{~Hz}, 1 \mathrm{H}), 3.44-3.30$ (m, $3 \mathrm{H}), 2.49(\mathrm{~s}, 3 \mathrm{H}), 2.20(\mathrm{~s}, 3 \mathrm{H})$.

${ }^{13} \mathrm{C}$ NMR $\left(100 \mathrm{MHz}, \mathrm{CDCl}_{3}\right) \delta 169.6,164.9,151.9,151.0,146.2,144.1,142.4,139.6$, $137.0,128.7,128.7,128.0,127.8,127.6,127.4,123.5,111.2,110.2,109.1,76.8,47.2$, $46.7,46.5,44.5,38.6,14.5,13.8$.

HRMS (ESI) $m / z$ calculated for $\mathrm{C}_{31} \mathrm{H}_{30} \mathrm{~N}_{3} \mathrm{O}_{4}{ }^{+}[\mathrm{M}+\mathrm{H}]^{+}:$508.2231, found 508.2224.

HPLC analysis: (IC column, Hexane:EtOH $=80: 20$, flow rate $=0.7 \mathrm{~mL} / \mathrm{min}$, wavelength $=254 \mathrm{~nm}$ ): $R \mathrm{t}=52.62$ (major), 35.02 (minor).

(2S,3R,4S)-6-benzyl-2-(2-(3,5-dimethyl-1H-pyrazol-1-yl)-2-oxoethyl)-4-phenyl-3-( thiophen-2-yl)-3,4,5,6-tetrahydropyrano[2,3-c]pyrrol-7(2H)-one (3ak) 


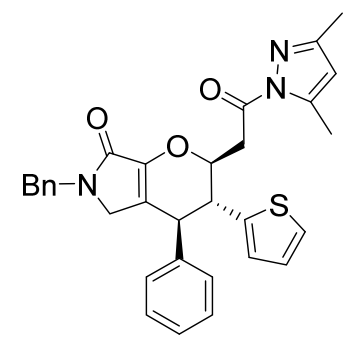

White solid, $93.2 \mathrm{mg}, 89 \%$ yield, $\mathrm{mp}=185.2-185.7{ }^{\circ} \mathrm{C},[\alpha] \mathrm{D}^{25}=-36.6($ c 0.904 , $\mathrm{CH}_{2} \mathrm{Cl}_{2}$ ), 98:2 dr, 95\% ee.

${ }^{1} \mathrm{H}$ NMR (400 MHz, $\left.\mathrm{CDCl}_{3}\right) \delta 7.29(\mathrm{q}, J=7.2 \mathrm{~Hz}, 3 \mathrm{H}), 7.22-7.16(\mathrm{~m}, 5 \mathrm{H}), 7.11(\mathrm{~d}$, $J=5.0 \mathrm{~Hz}, 1 \mathrm{H}), 6.93(\mathrm{~d}, J=5.5 \mathrm{~Hz}, 2 \mathrm{H}), 6.80-6.74(\mathrm{~m}, 1 \mathrm{H}), 6.62(\mathrm{~d}, J=3.0 \mathrm{~Hz}$, $1 \mathrm{H}), 5.92(\mathrm{~s}, 1 \mathrm{H}), 5.01(\mathrm{t}, J=7.6 \mathrm{~Hz}, 1 \mathrm{H}), 4.87(\mathrm{~d}, J=15.0 \mathrm{~Hz}, 1 \mathrm{H}), 4.32(\mathrm{~d}, J=15.0$ $\mathrm{Hz}, 1 \mathrm{H}), 3.86(\mathrm{~d}, J=10.1 \mathrm{~Hz}, 1 \mathrm{H}), 3.66(\mathrm{dd}, J=17.6,7.8 \mathrm{~Hz}, 1 \mathrm{H}), 3.60-3.46(\mathrm{~m}$, $2 \mathrm{H}), 3.41-3.25(\mathrm{~m}, 2 \mathrm{H}), 2.51(\mathrm{~s}, 3 \mathrm{H}), 2.19(\mathrm{~s}, 3 \mathrm{H})$.

${ }^{13} \mathrm{C}$ NMR $\left(100 \mathrm{MHz}, \mathrm{CDCl}_{3}\right) \delta 170.0,164.9,151.9,146.2,144.2,141.3,139.5,137.1$, $128.8,128.7,128.7,128.0,127.6,127.4,126.9,126.4,124.9,123.6,123.5,111.2$, $78.4,49.0,48.2,47.2,46.5,38.6,14.6,13.8$.

HRMS (ESI) $m / z$ calculated for $\mathrm{C}_{31} \mathrm{H}_{30} \mathrm{~N}_{3} \mathrm{O}_{3} \mathrm{~S}^{+}[\mathrm{M}+\mathrm{H}]^{+}:$: 524.2002, found 524.2001.

HPLC analysis: (IC column, Hexane:2-propanol $=40: 60$, flow rate $=0.5 \mathrm{~mL} / \mathrm{min}$, wavelength $=254 \mathrm{~nm}$ ): $R \mathrm{t}=58.99$ (major), 47.74 (minor).

(2R,4S)-6-benzyl-2-(2-(3,5-dimethyl-1H-pyrazol-1-yl)-2-oxoethyl)-4-phenyl-3,4,5, 6-tetrahydropyrano[2,3-c]pyrrol-7(2H)-one (3al)<smiles>Cc1cc(C)n(C(=O)CC2CC(c3ccccc3)[C@@H]3CN(Cc4ccccc4)C(=O)C23)n1</smiles>

White solid, $42.3 \mathrm{mg}, 48 \%$ yield, $\mathrm{mp}=174.2-174.7{ }^{\circ} \mathrm{C},[\alpha] \mathrm{D}^{25}=-12.7($ c 0.423 , $\mathrm{CH}_{2} \mathrm{Cl}_{2}$ ), 90:10 dr, $>99 \%$ ee.

${ }^{1} \mathrm{H}$ NMR (400 MHz, $\left.\mathrm{CDCl}_{3}\right) \delta 7.32-7.22(\mathrm{~m}, 6 \mathrm{H}), 7.18(\mathrm{dd}, J=6.6,1.6 \mathrm{~Hz}, 2 \mathrm{H})$, $7.14-7.08(\mathrm{~m}, 2 \mathrm{H}), 5.96(\mathrm{~s}, 1 \mathrm{H}), 4.81(\mathrm{t}, J=12.9 \mathrm{~Hz}, 2 \mathrm{H}), 4.33(\mathrm{~d}, J=15.0 \mathrm{~Hz}, 1 \mathrm{H})$, $3.83(\mathrm{dd}, J=17.3,6.0 \mathrm{~Hz}, 2 \mathrm{H}), 3.48-3.38(\mathrm{~m}, 2 \mathrm{H}), 2.53(\mathrm{~s}, 3 \mathrm{H}), 2.41(\mathrm{dd}, J=14.7$, $7.0 \mathrm{~Hz}, 1 \mathrm{H}), 2.23$ (d, $J=12.7 \mathrm{~Hz}, 4 \mathrm{H}), 2.02-1.88(\mathrm{~m}, 1 \mathrm{H})$.

${ }^{13} \mathrm{C} \mathrm{NMR}\left(100 \mathrm{MHz}, \mathrm{CDCl}_{3}\right) \delta 170.1,165.2,152.2,146.1,144.2,142.6,137.1,128.9$, $128.7,128.1,127.8,127.60127 .1,120.8,111.4,70.5,47.9,46.5,39.9,36.4,35.7$, $14.5,13.8$.

HRMS (ESI) $m / z$ calculated for $\mathrm{C}_{27} \mathrm{H}_{28} \mathrm{~N}_{3} \mathrm{O}_{3}{ }^{+}[\mathrm{M}+\mathrm{H}]^{+}: 442.2125$, found 442.2124. 
HPLC analysis: (AD-H column, Hexane:2-propanol $=50: 50$, flow rate $=0.5 \mathrm{~mL} / \mathrm{min}$, wavelength $=254 \mathrm{~nm}$ ): $R \mathrm{t}=22.30$ (major), 39.57 (minor).

(2S,3R,4S)-6-benzyl-2-(2-(3,5-dimethyl-1H-pyrazol-1-yl)-2-oxoethyl)-3-methyl-4phenyl-3,4,5,6-tetrahydropyrano[2,3-c]pyrrol-7(2H)-one (3am)<smiles>Cc1cc(C)n(C(=O)C[C@H]2OC3=C(CN(Cc4ccccc4)C3=O)[C@@H](c3ccccc3)[C@H]2C)n1</smiles>

White solid, $73.0 \mathrm{mg}, 80 \%$ yield, $\mathrm{mp}=128.2-129.7{ }^{\circ} \mathrm{C},[\alpha] \mathrm{D}^{25}=-31.0$ (c 0.730 , $\mathrm{CH}_{2} \mathrm{Cl}_{2}$ ), 85:15 dr, $>99 \%$ ee.

${ }^{1} \mathrm{H}$ NMR $\left(400 \mathrm{MHz}, \mathrm{CDCl}_{3}\right) \delta 7.32-7.22(\mathrm{~m}, 7 \mathrm{H}), 7.18-7.14(\mathrm{~m}, 2 \mathrm{H}), 7.12-7.08$ $(\mathrm{m}, 2 \mathrm{H}), 5.97(\mathrm{~s}, 1 \mathrm{H}), 4.81(\mathrm{~d}, \mathrm{~J}=15.0 \mathrm{~Hz}, 1 \mathrm{H}), 4.56(\mathrm{~m}, \mathrm{~J}=10.7,7.4,3.9 \mathrm{~Hz}, 1 \mathrm{H})$, $4.29(\mathrm{~d}, \mathrm{~J}=15.1 \mathrm{~Hz}, 1 \mathrm{H}), 3.70(\mathrm{dd}, \mathrm{J}=17.4,7.4 \mathrm{~Hz}, 1 \mathrm{H}), 3.57(\mathrm{dd}, \mathrm{J}=17.4,4.0 \mathrm{~Hz}$, 1H), $3.43(\mathrm{~d}, \mathrm{~J}=19.3 \mathrm{~Hz}, 1 \mathrm{H}), 3.29-3.20(\mathrm{~m}, 2 \mathrm{H}), 2.57$ (s, 3H), 2.23 (s, 3H), 2.17 $2.10(\mathrm{~m}, 1 \mathrm{H}), 0.91(\mathrm{~d}, \mathrm{~J}=6.7 \mathrm{~Hz}, 3 \mathrm{H})$.

${ }^{13} \mathrm{C}$ NMR $\left(100 \mathrm{MHz}, \mathrm{CDCl}_{3}\right) \delta 170.5,165.2,152.1,145.8,144.3,140.5,137.1,128.8$, $128.7,128.4,128.0,127.5,127.4,123.6,111.3,79.1,47.3,47.0,46.4,39.8,38.6,14.7$, $14.6,13.8$.

HPLC analysis: (IA column, Hexane:2-propanol $=90: 10$, flow rate $=0.4 \mathrm{~mL} / \mathrm{min}$, wavelength $=254 \mathrm{~nm}$ ): $R \mathrm{t}=130.60$ (major), 125.44 (minor).

(2S,3R,4S)-3,6-dibenzyl-2-(2-(3,5-dimethyl-1H-pyrazol-1-yl)-2-oxoethyl)-4-phenyl $-3,4,5,6$-tetrahydropyrano[2,3-c]pyrrol-7(2H)-one (3an)<smiles>Cc1cc(C)n(C(=O)CC2CC3=C(O[C@H](Cc4ccccc4)C3c3ccccc3)C(=O)O2)n1</smiles>

White solid, $47.1 \mathrm{mg}, 44 \%$ yield, $\mathrm{mp}=173.4-174.5{ }^{\circ} \mathrm{C},[\alpha] \mathrm{D}^{25}=-28.7$ (c 0.471, $\mathrm{CH}_{2} \mathrm{Cl}_{2}$ ), 80:20 dr, $>99 \%$ ee.

${ }^{1} \mathrm{H}$ NMR $\left(400 \mathrm{MHz}, \mathrm{CDCl}_{3}\right) \delta 7.28(\mathrm{~m}, \mathrm{~J}=13.8,7.6,6.8,1.7 \mathrm{~Hz}, 5 \mathrm{H}), 7.21-7.15(\mathrm{~m}$, $5 \mathrm{H}), 7.11(\mathrm{dd}, \mathrm{J}=6.2,2.2 \mathrm{~Hz}, 3 \mathrm{H}), 7.02(\mathrm{~d}, \mathrm{~J}=6.9 \mathrm{~Hz}, 2 \mathrm{H}), 5.89(\mathrm{~s}, 1 \mathrm{H}), 4.83-4.72$ $(\mathrm{m}, 2 \mathrm{H}), 4.36(\mathrm{~d}, \mathrm{~J}=15.0 \mathrm{~Hz}, 1 \mathrm{H}), 3.53(\mathrm{dd}, \mathrm{J}=17.3,7.5 \mathrm{~Hz}, 1 \mathrm{H}), 3.48-3.35(\mathrm{~m}$, $4 \mathrm{H}), 2.91-2.82(\mathrm{~m}, 1 \mathrm{H}), 2.72-2.64(\mathrm{~m}, 2 \mathrm{H}), 2.47(\mathrm{~s}, 3 \mathrm{H}), 2.17(\mathrm{~s}, 3 \mathrm{H})$. 
${ }^{13} \mathrm{C} \mathrm{NMR}\left(100 \mathrm{MHz}, \mathrm{CDCl}_{3}\right) \delta 170.3,165.1,151.7,144.9,144.0,140.8,138.7,137.1$, $129.1,128.9,128.7,128.4,128.3,128.0,127.5,127.2,126.4,122.1,111.1,77.6,47.8$, $46.5,44.8,43.0,39.0,36.7,14.5,13.8$.

HPLC analysis: (AD-H column, Hexane:EtOH $=90: 10$, flow rate $=0.3 \mathrm{~mL} / \mathrm{min}$, wavelength $=254 \mathrm{~nm}$ ): $R \mathrm{t}=257.24$ (major), 82.29 (minor).

(2S,3S,4S)-6-benzyl-2-(2-oxo-2-(1H-pyrazol-1-yl)ethyl)-3,4-diphenyl-3,4,5,6-tetra hydropyrano[2,3-c]pyrrol-7(2H)-one (3ao)<smiles>O=C1OC2=C(CN1Cc1ccccc1)[C@H](c1ccccc1)[C@H](CC(=O)n1cccn1)C2</smiles>

White solid, $33.1 \mathrm{mg}, 34 \%$ yield, $\mathrm{mp}=165.2-165.7{ }^{\circ} \mathrm{C},[\alpha] \mathrm{D}^{25}=-12.2($ c 0.331 , $\mathrm{CH}_{2} \mathrm{Cl}_{2}$ ), 90:10 dr, 93\% ee.

${ }^{1} \mathrm{H}$ NMR $\left(400 \mathrm{MHz}, \mathrm{CDCl}_{3}\right) \delta 8.16(\mathrm{~s}, 1 \mathrm{H}), 7.62(\mathrm{~s}, 1 \mathrm{H}), 7.33-7.26(\mathrm{~m}, 3 \mathrm{H}), 7.22-$ $7.18(\mathrm{~m}, 2 \mathrm{H}), 7.17-7.11(\mathrm{~m}, 6 \mathrm{H}), 6.95(\mathrm{~d}, \mathrm{~J}=6.5 \mathrm{~Hz}, 2 \mathrm{H}), 6.83(\mathrm{~d}, \mathrm{~J}=7.2 \mathrm{~Hz}, 2 \mathrm{H})$, 6.37 (s, 1H), $5.10(\mathrm{t}, \mathrm{J}=7.9 \mathrm{~Hz}, 1 \mathrm{H}), 4.86(\mathrm{~d}, \mathrm{~J}=15.0 \mathrm{~Hz}, 1 \mathrm{H}), 4.34$ (d, J = 15.0 Hz, $1 \mathrm{H}), 3.85(\mathrm{~d}, \mathrm{~J}=10.2 \mathrm{~Hz}, 1 \mathrm{H}), 3.62(\mathrm{dd}, \mathrm{J}=17.5,8.2 \mathrm{~Hz}, 1 \mathrm{H}), 3.53(\mathrm{~d}, \mathrm{~J}=16.8 \mathrm{~Hz}$, $1 \mathrm{H}), 3.40(\mathrm{~d}, \mathrm{~J}=18.1 \mathrm{~Hz}, 1 \mathrm{H}), 3.17-3.08(\mathrm{~m}, 2 \mathrm{H})$.

${ }^{13} \mathrm{C}$ NMR $\left(100 \mathrm{MHz}, \mathrm{CDCl}_{3}\right) \delta 168.6,164.9,146.1,143.9,139.5,138.4,137.0,128.8$, $128.7,128.6,128.4,128.0,128.0,127.6,127.5,127.2,124.1,109.7,77.7,53.0,47.5$, $47.2,46.5,37.7$.

HPLC analysis: (AD-H column, Hexane:2-propanol $=80: 20$, flow rate $=0.6 \mathrm{~mL} / \mathrm{min}$, wavelength $=254 \mathrm{~nm}$ ): $R \mathrm{t}=22.25$ (major), 28.11 (minor).

(2S,3S,4S)-2-(2-(1H-benzo[d] [1,2,3]triazol-1-yl)-2-oxoethyl)-6-benzyl-3,4-dipheny $1-3,4,5,6$-tetrahydropyrano[2,3-c]pyrrol-7(2H)-one (3ap)<smiles>O=C1OC(CC(=O)n2nnc3ccccc32)C(c2ccccc2)C(c2ccccc2)C1=O</smiles>

White solid, $72.7 \mathrm{mg}, 67 \%$ yield, $\mathrm{mp}=119.2-120.7{ }^{\circ} \mathrm{C},[\alpha] \mathrm{D}^{25}=-38.8($ c 0.727 , $\mathrm{CH}_{2} \mathrm{Cl}_{2}$ ), 86:14 dr, $>99 \%$ ee. 
${ }^{1} \mathrm{H}$ NMR $\left(400 \mathrm{MHz}, \mathrm{CDCl}_{3}\right) \delta 8.12(\mathrm{~d}, \mathrm{~J}=7.5 \mathrm{~Hz}, 1 \mathrm{H}), 7.99(\mathrm{~d}, \mathrm{~J}=7.4 \mathrm{~Hz}, 1 \mathrm{H}), 7.54$ $(\mathrm{t}, \mathrm{J}=7.6 \mathrm{~Hz}, 1 \mathrm{H}), 7.40(\mathrm{t}, \mathrm{J}=7.6 \mathrm{~Hz}, 1 \mathrm{H}), 7.20(\mathrm{dd}, \mathrm{J}=15.1,6.5 \mathrm{~Hz}, 3 \mathrm{H}), 7.12(\mathrm{~d}, \mathrm{~J}$ $=7.1 \mathrm{~Hz}, 2 \mathrm{H}), 7.08-7.01(\mathrm{~m}, 5 \mathrm{H}), 6.93(\mathrm{dd}, \mathrm{J}=22.0,6.7 \mathrm{~Hz}, 3 \mathrm{H}), 6.77(\mathrm{~s}, 2 \mathrm{H}), 5.12$ $(\mathrm{s}, 1 \mathrm{H}), 4.79(\mathrm{~d}, \mathrm{~J}=15.0 \mathrm{~Hz}, 1 \mathrm{H}), 4.27(\mathrm{~d}, \mathrm{~J}=15.0 \mathrm{~Hz}, 1 \mathrm{H}), 3.85-3.69(\mathrm{~m}, 2 \mathrm{H}), 3.52$ $-3.31(\mathrm{~m}, 3 \mathrm{H}), 3.12(\mathrm{t}, \mathrm{J}=10.2 \mathrm{~Hz}, 1 \mathrm{H})$.

${ }^{13} \mathrm{C}$ NMR $\left(100 \mathrm{MHz}, \mathrm{CDCl}_{3}\right) \delta 168.7,164.8,146.1,146.0,139.3,138.1,137.0,130.0$, $131.39,129.0,128.7,128.6,128.0,128.0,127.6,127.6,127.3,126.2,124.3,120.1$, $114.5,77.6,53.1,47.4,47.3,46.5,39.3$.

HPLC analysis: (AD-H column, Hexane:2-propanol $=90: 10$, flow rate $=0.4 \mathrm{~mL} / \mathrm{min}$, wavelength $=254 \mathrm{~nm}$ ): $R \mathrm{t}=117.19$ (major), 109.67 (minor).

\subsection{Gram-scale of $3 a a$}

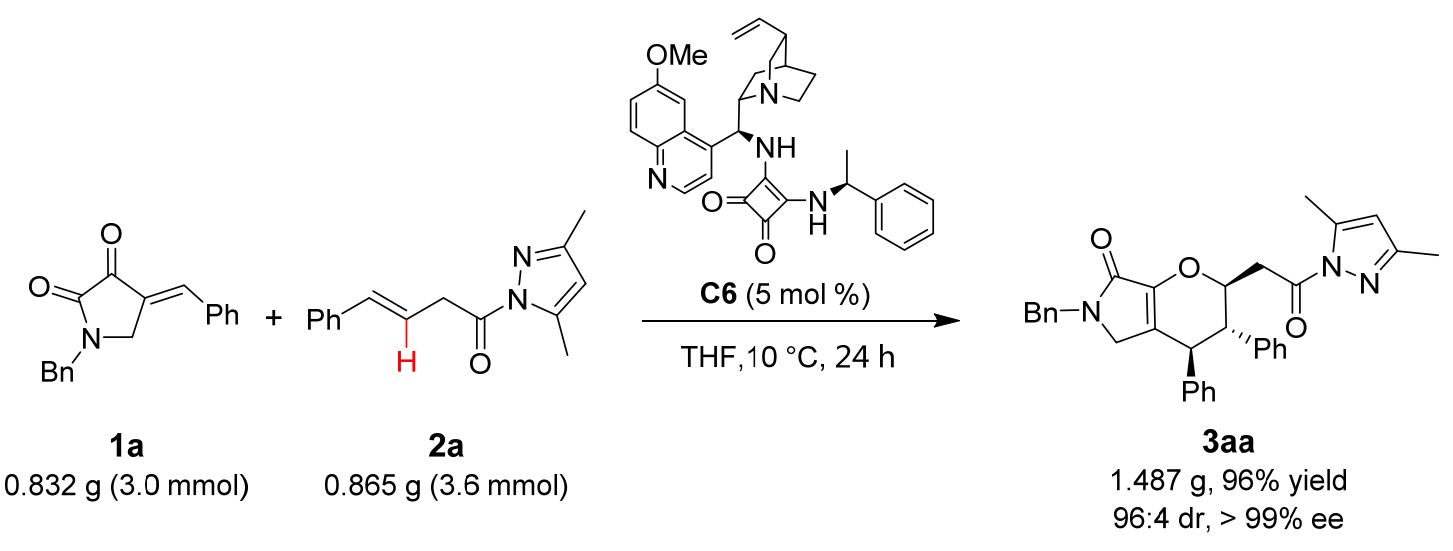

\section{General procedure for the 1,2 -selective $\gamma$-addition cyclization reaction}

\subsection{Synthesis of 5}

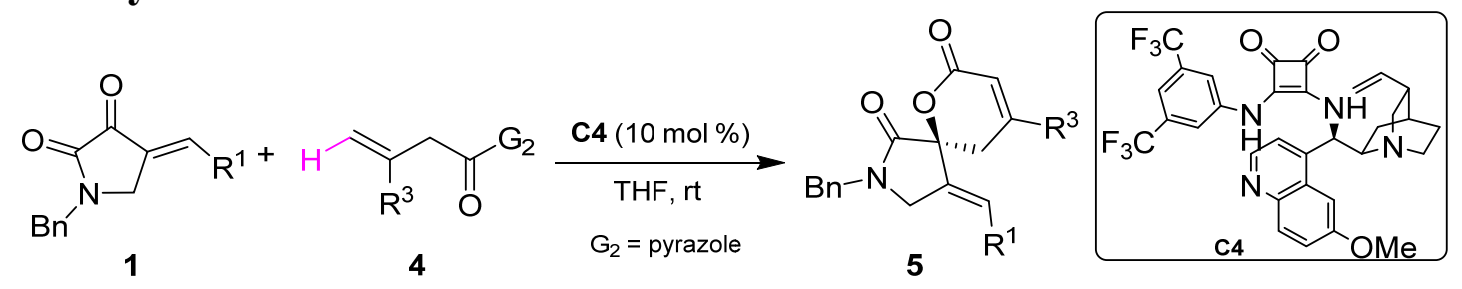

Unsaturated ketone 1 ( $0.1 \mathrm{mmol}, 1.0$ equiv.) and catalyst $\mathbf{C 4}$ (0.01 mmol, 0.1 equiv.) were dissolved in THF ( $0.5 \mathrm{~mL})$, then $N$-acylpyrazole 4 ( $0.2 \mathrm{mmol} 2.0$ equiv.) was added. The reaction mixture was stirred at room temperature for 24-48 hours. After removal of the solvent under reduced pressure, the reaction mixture was 
purified by flash chromatography on silica gel (hexane/EtOAc $=3.5 / 1)$ to give the pure product 5 .

(S,E)-2-benzyl-4-benzylidene-9-phenyl-6-oxa-2-azaspiro[4.5]dec-8-ene-1,7-dione (5aa)<smiles>O=C1C=C(c2ccccc2)CC2(C=C(c3ccccc3)CN2Cc2ccccc2)C1=O</smiles>

White solid, $42.0 \mathrm{mg}, 99 \%$ yield, $\mathrm{mp}=204.5-205.4{ }^{\circ} \mathrm{C},[\alpha] \mathrm{D}^{25}=-1.4$ (c 0.421 , $\mathrm{CH}_{2} \mathrm{Cl}_{2}$ ), $99 \%$ ee.

${ }^{1} \mathrm{H}$ NMR (400 MHz, $\left.\mathrm{CDCl}_{3}\right) \delta 7.66-7.56(\mathrm{~m}, 2 \mathrm{H}), 7.53-7.44(\mathrm{~m}, 3 \mathrm{H}), 7.43-7.25$ (m, 8H), 7.17 (d, J=7.2 Hz, 2H), $6.93(\mathrm{~s}, 1 \mathrm{H}), 6.55(\mathrm{~s}, 1 \mathrm{H}), 4.59(\mathrm{~s}, 2 \mathrm{H}), 4.33-4.13$ (m, 2H), $3.42(\mathrm{~d}, J=17.9 \mathrm{~Hz}, 1 \mathrm{H}), 3.18(\mathrm{~d}, J=18.0 \mathrm{~Hz}, 1 \mathrm{H})$.

${ }^{13} \mathrm{C}$ NMR $\left(100 \mathrm{MHz}, \mathrm{CDCl}_{3}\right) \delta 169.3,163.6,151.2,136.1,135.1,134.6,132.6,130.8$, $129.1,129.0,128.8,128.7,128.7,128.5,128.2,128.1,114.9,81.9,48.3,47.0,33.5$.

HRMS (ESI) $\mathrm{m} / \mathrm{z}$ calculated for $\mathrm{C}_{28} \mathrm{H}_{24} \mathrm{NO}_{3}{ }^{+}[\mathrm{M}+\mathrm{H}]^{+}: 422.1751$, found 422.1751 .

HPLC analysis: (IB column, Hexane:2-propanol $=80: 20$, flow rate $=1.0 \mathrm{~mL} / \mathrm{min}$, wavelength $=254 \mathrm{~nm}$ ): $R \mathrm{t}=40.11$ (major), 34.60 (minor).

(S,E)-2-benzyl-4-(2-chlorobenzylidene)-9-phenyl-6-oxa-2-azaspiro[4.5]dec-8-ene1,7-dione (5ba)<smiles>CC12C(=O)N(Cc3ccccc3)CC1(/C=C/c1ccccc1Cl)OC(=O)C=C2c1ccccc1</smiles>

White solid, $42.7 \mathrm{mg}, 94 \%$ yield, $\mathrm{mp}=184.5-185.3{ }^{\circ} \mathrm{C},[\alpha] \mathrm{D}^{25}=21.5$ (c 0.417 , $\mathrm{CH}_{2} \mathrm{Cl}_{2}$ ), $90 \%$ ee.

${ }^{1} \mathrm{H}$ NMR (400 MHz, $\left.\mathrm{CDCl}_{3}\right) \delta 7.64-7.57(\mathrm{~m}, 2 \mathrm{H}), 7.47(\mathrm{~d}, J=4.2 \mathrm{~Hz}, 3 \mathrm{H}), 7.33(\mathrm{dt}$, $J=11.2,6.4 \mathrm{~Hz}, 5 \mathrm{H}), 7.23(\mathrm{dd}, J=8.8,4.0 \mathrm{~Hz}, 3 \mathrm{H}), 7.16(\mathrm{~s}, 1 \mathrm{H}), 7.13-7.08(\mathrm{~m}, 1 \mathrm{H})$, $6.50(\mathrm{~s}, 1 \mathrm{H}), 4.62(\mathrm{~d}, J=14.7 \mathrm{~Hz}, 1 \mathrm{H}), 4.50(\mathrm{~d}, J=14.7 \mathrm{~Hz}, 1 \mathrm{H}), 4.24(\mathrm{~d}, J=13.9 \mathrm{~Hz}$, 1H), 3.94 (d, $J=14.1 \mathrm{~Hz}, 1 \mathrm{H}), 3.50$ (d, $J=18.0 \mathrm{~Hz}, 1 \mathrm{H}), 3.19$ (d, $J=18.0 \mathrm{~Hz}, 1 \mathrm{H})$. 
${ }^{13} \mathrm{C} \mathrm{NMR}\left(100 \mathrm{MHz}, \mathrm{CDCl}_{3}\right) \delta 169.0,163.2,152.3,136.1,134.9,134.8,134.0,132.6$, $130.9,129.9,129.8,129.1,129.1,129.0,128.2,128.1,126.9,126.2,125.7,114.9$, $81.8,47.9,47.0,325$.

HRMS (ESI) $m / z$ calculated for $\mathrm{C}_{28} \mathrm{H}_{23} \mathrm{ClNO}_{3}{ }^{+}[\mathrm{M}+\mathrm{H}]^{+}: 456.1361$, found 456.1355 .

HPLC analysis: (IA column, Hexane:2-propanol $=75: 25$, flow rate $=0.7 \mathrm{~mL} / \mathrm{min}$, wavelength $=254 \mathrm{~nm}$ ): $R \mathrm{t}=27.66$ (major), 21.84 (minor).

(S,E)-2-benzyl-4-(2-bromobenzylidene)-9-phenyl-6-oxa-2-azaspiro[4.5]dec-8-ene1,7-dione (5ca)<smiles>CC1=CC(=O)OC1(C)C(=Cc1ccccc1Br)CN(Cc1ccccc1)C(=O)C=Cc1ccccc1</smiles>

White solid, $47.9 \mathrm{mg}, 96 \%$ yield, $\mathrm{mp}=184.9-185.9{ }^{\circ} \mathrm{C},[\alpha] \mathrm{D}^{25}=25.5$ (c 0.468 , $\left.\mathrm{CH}_{2} \mathrm{Cl}_{2}\right), 91 \%$ ee.

${ }^{1} \mathrm{H}$ NMR (400 MHz, $\left.\mathrm{CDCl}_{3}\right) \delta 7.66-7.60(\mathrm{~m}, 2 \mathrm{H}), 7.55$ (d, J= 7.9 Hz, 1H), 7.47 (d, $J=4.6 \mathrm{~Hz}, 3 \mathrm{H}), 7.33(\mathrm{dq}, J=14.2,6.7 \mathrm{~Hz}, 4 \mathrm{H}), 7.25(\mathrm{~s}, 1 \mathrm{H}), 7.20-7.05(\mathrm{~m}, 4 \mathrm{H})$, $6.51(\mathrm{~s}, 1 \mathrm{H}), 4.64(\mathrm{~d}, J=14.7 \mathrm{~Hz}, 1 \mathrm{H}), 4.49$ (d, $J=14.8 \mathrm{~Hz}, 1 \mathrm{H}), 4.23$ (d, J=14.0 Hz, $1 \mathrm{H}), 3.90$ (d, $J=14.2 \mathrm{~Hz}, 1 \mathrm{H}), 3.53$ (d, $J=18.0 \mathrm{~Hz}, 1 \mathrm{H}), 3.20$ (d, $J=18.1 \mathrm{~Hz}, 1 \mathrm{H})$.

${ }^{13} \mathrm{C}$ NMR $\left(100 \mathrm{MHz}, \mathrm{CDCl}_{3}\right) \delta 169.0,163.2,152.5,136.0,134.9,134.7,134.3,133.1$, $130.9,130.0,129.1,129.1,129.0,128.2,128.2,128.1,127.6,126.3,124.3,114.8$, $81.7,47.7,47.0,32.3$.

HRMS (ESI) $\mathrm{m} / \mathrm{z}$ calculated for $\mathrm{C}_{28} \mathrm{H}_{22} \mathrm{BrNNaO}_{3}{ }^{+}[\mathrm{M}+\mathrm{Na}]^{+}: 522.0675$, found 522.0669 .

HPLC analysis: (IA column, Hexane:2-propanol $=75: 25$, flow rate $=0.7 \mathrm{~mL} / \mathrm{min}$, wavelength $=254 \mathrm{~nm}$ ): $R \mathrm{t}=27.78$ (major), 22.35 (minor).

(S,E)-2-benzyl-4-(2-methylbenzylidene)-9-phenyl-6-oxa-2-azaspiro[4.5]dec-8-ene1,7-dione (5da)<smiles>Cc1ccccc1/C=C1\CN(Cc2ccccc2)C(=O)C1(C)C1=CC(=O)OC(=O)C1</smiles>

White solid, $39.2 \mathrm{mg}, 90 \%$ yield, $\mathrm{mp}=191.0-191.6{ }^{\circ} \mathrm{C},[\alpha] \mathrm{D}^{25}=-1.4$ (c 0.382 , $\mathrm{CH}_{2} \mathrm{Cl}_{2}$ ), 96\% ee. 
${ }^{1} \mathrm{H}$ NMR (400 MHz, $\left.\mathrm{CDCl}_{3}\right) \delta 7.59(\mathrm{dd}, J=6.5,2.9 \mathrm{~Hz}, 2 \mathrm{H}), 7.47(\mathrm{dd}, J=7.1,3.6 \mathrm{~Hz}$, $3 \mathrm{H}), 7.33(\mathrm{td}, J=10.0,4.4 \mathrm{~Hz}, 3 \mathrm{H}), 7.27-7.22(\mathrm{~m}, 2 \mathrm{H}), 7.21-7.11(\mathrm{~m}, 3 \mathrm{H}), 7.06-$ $6.98(\mathrm{~m}, 2 \mathrm{H}), 6.52(\mathrm{~s}, 1 \mathrm{H}), 4.61-4.48(\mathrm{~m}, 2 \mathrm{H}), 4.14(\mathrm{dd}, J=14.2,2.0 \mathrm{~Hz}, 1 \mathrm{H}), 3.99$ (dd, $J=14.2,2.0 \mathrm{~Hz}, 1 \mathrm{H}), 3.47$ (d, $J=16.9 \mathrm{~Hz}, 1 \mathrm{H}), 3.14$ (d, $J=17.9 \mathrm{~Hz}, 1 \mathrm{H}), 2.19$ $(\mathrm{s}, 3 \mathrm{H})$.

${ }^{13} \mathrm{C}$ NMR $\left(100 \mathrm{MHz}, \mathrm{CDCl}_{3}\right) \delta 169.3,163.5,151.6,136.8,136.1,135.1,133.4,133.3$, $130.9,130.5,129.1,129.0,128.6,128.2,128.1,127.7,127.1,126.1,126.0,114.9$, $81.8,47.9,47.0,33.1,19.8$.

HRMS (ESI) $m / z$ calculated for $\mathrm{C}_{29} \mathrm{H}_{25} \mathrm{NNaO}_{3}{ }^{+}[\mathrm{M}+\mathrm{Na}]^{+}:$458.1727, found 458.1720.

HPLC analysis: (IA column, Hexane:2-propanol $=75: 25$, flow rate $=0.7 \mathrm{~mL} / \mathrm{min}$, wavelength $=254 \mathrm{~nm}$ ): $R \mathrm{t}=23.16$ (major), 17.64 (minor).

(S,E)-2-benzyl-4-(3-fluorobenzylidene)-9-phenyl-6-oxa-2-azaspiro[4.5]dec-8-ene1,7-dione (5ea)<smiles>O=C1C=C(c2ccccc2)CC2(C=C(C=Cc3cccc(F)c3)CN(Cc3ccccc3)C2=O)O1</smiles>

White solid, $43.5 \mathrm{mg}, 99 \%$ yield, $\mathrm{mp}=183.0-183.8{ }^{\circ} \mathrm{C},[\alpha] \mathrm{D}^{25}=-3.5($ c 0.438 , $\left.\mathrm{CH}_{2} \mathrm{Cl}_{2}\right), 97 \%$ ee.

${ }^{1} \mathrm{H}$ NMR $\left(400 \mathrm{MHz}, \mathrm{CDCl}_{3}\right) \delta 7.61-7.54(\mathrm{~m}, 2 \mathrm{H}), 7.46(\mathrm{~d}, J=4.9 \mathrm{~Hz}, 3 \mathrm{H}), 7.33(\mathrm{dt}$, $J=10.8,6.7 \mathrm{~Hz}, 4 \mathrm{H}), 7.27(\mathrm{~s}, 1 \mathrm{H}), 7.25(\mathrm{~s}, 1 \mathrm{H}), 7.02-6.91(\mathrm{~m}, 2 \mathrm{H}), 6.90-6.80(\mathrm{~m}$, $2 \mathrm{H}), 6.52(\mathrm{~s}, 1 \mathrm{H}), 4.56(\mathrm{~s}, 2 \mathrm{H}), 4.29-4.11(\mathrm{~m}, 2 \mathrm{H}), 3.38(\mathrm{~d}, J=18.0 \mathrm{~Hz}, 1 \mathrm{H}), 3.14$ (d, $J=18.0 \mathrm{~Hz}, 1 \mathrm{H})$.

${ }^{13} \mathrm{C}$ NMR $\left(100 \mathrm{MHz}, \mathrm{CDCl}_{3}\right) \delta 169.1,164.0,163.5,161.5,151.0,136.7,136.6,135.9$, $134.9,134.1,130.9,130.4,130.4,129.1,129.1,128.2,128.2,127.6,127.5,126.1$, $124.6,124.6,115.5,115.4,115.3,115.2,114.8,81.7,48.1,47.0,33.6$.

HRMS (ESI) $m / z$ calculated for $\mathrm{C}_{28} \mathrm{H}_{23} \mathrm{FNO}_{3}{ }^{+}[\mathrm{M}+\mathrm{H}]^{+}:$440.1657, found 440.1646.

HPLC analysis: (IA column, Hexane:2-propanol $=75: 25$, flow rate $=0.7 \mathrm{~mL} / \mathrm{min}$, wavelength $=254 \mathrm{~nm}$ ): $R \mathrm{t}=31.19$ (major), 22.80 (minor).

(S,E)-2-benzyl-4-(3-bromobenzylidene)-9-phenyl-6-oxa-2-azaspiro[4.5]dec-8-ene1,7-dione (5fa) 


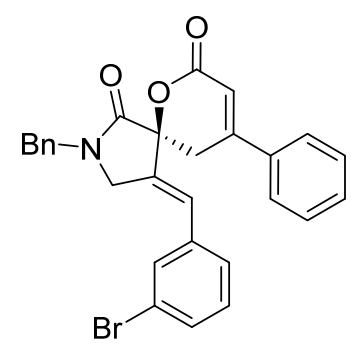

White solid, $48.9 \mathrm{mg}, 98 \%$ yield, $\mathrm{mp}=172.4-173.2{ }^{\circ} \mathrm{C},[\alpha] \mathrm{D}^{25}=-3.0$ (c 0.500 , $\left.\mathrm{CH}_{2} \mathrm{Cl}_{2}\right), 97 \%$ ee.

${ }^{1} \mathrm{H}$ NMR (400 MHz, $\left.\mathrm{CDCl}_{3}\right) \delta 7.65-7.56(\mathrm{~m}, 2 \mathrm{H}), 7.50(\mathrm{~s}, 3 \mathrm{H}), 7.42-7.22(\mathrm{~m}, 7 \mathrm{H})$, $7.01(\mathrm{t}, J=8.2 \mathrm{~Hz}, 1 \mathrm{H}), 6.96(\mathrm{~d}, J=7.6 \mathrm{~Hz}, 1 \mathrm{H}), 6.90(\mathrm{~s}, 1 \mathrm{H}), 6.86(\mathrm{~d}, J=9.8 \mathrm{~Hz}$, $1 \mathrm{H}), 6.55(\mathrm{~s}, 1 \mathrm{H}), 4.59(\mathrm{~s}, 2 \mathrm{H}), 4.26(\mathrm{~d}, J=14.2 \mathrm{~Hz}, 1 \mathrm{H}), 4.16(\mathrm{~d}, J=14.2 \mathrm{~Hz}, 1 \mathrm{H})$, $3.41(\mathrm{~d}, J=17.9 \mathrm{~Hz}, 1 \mathrm{H}), 3.16(\mathrm{~d}, J=17.9 \mathrm{~Hz}, 1 \mathrm{H})$.

${ }^{13} \mathrm{C}$ NMR $\left(100 \mathrm{MHz}, \mathrm{CDCl}_{3}\right) \delta 169.1,163.4,151.0,136.7,136.6,136.0,134.9,134.1$, $130.9,130.4$, 130.3, 129.1, 129.1, 129.1, 129.1, 128.2, 128.2, 128.2, 127.5, 127.5, 126.1, 124.6, 124.6, 114.9, 81.7, 48.1, 47.0, 33.6.

HRMS (ESI) $\mathrm{m} / z$ calculated for $\mathrm{C}_{28} \mathrm{H}_{23} \mathrm{BrNO}_{3}{ }^{+}[\mathrm{M}+\mathrm{H}]^{+}: 500.0856$, found 500.0849 .

HPLC analysis: (IA column, Hexane:2-propanol $=75: 25$, flow rate $=1.0 \mathrm{~mL} / \mathrm{min}$, wavelength $=254 \mathrm{~nm}$ ): $R \mathrm{t}=22.05$ (major), 17.55 (minor).

(S,E)-2-benzyl-4-(3-methoxybenzylidene)-9-phenyl-6-oxa-2-azaspiro[4.5]dec-8-en e-1,7-dione (5ga)<smiles>COc1cccc(/C=C2\CN(Cc3ccccc3)C(=O)C2(Cc2ccccc2)OC(=O)C=Cc2ccccc2)c1</smiles>

White solid, $44.3 \mathrm{mg}, 98 \%$ yield, $\mathrm{mp}=181.3-182.5{ }^{\circ} \mathrm{C},[\alpha] \mathrm{D}^{25}=-2.9$ (c 0.501 , $\mathrm{CH}_{2} \mathrm{Cl}_{2}$ ), $95 \%$ ee.

${ }^{1} \mathrm{H}$ NMR (400 MHz, $\left.\mathrm{CDCl}_{3}\right) \delta 7.57(\mathrm{~s}, 2 \mathrm{H}), 7.45$ (s, 3H), $7.31(\mathrm{~s}, 4 \mathrm{H}), 7.25(\mathrm{~s}, 2 \mathrm{H})$, $6.91-6.78(\mathrm{~m}, 2 \mathrm{H}), 6.77-6.64(\mathrm{~m}, 2 \mathrm{H}), 6.51(\mathrm{~s}, 1 \mathrm{H}), 4.54(\mathrm{~s}, 2 \mathrm{H}), 4.20(\mathrm{q}, J=13.5$ $\mathrm{Hz}, 2 \mathrm{H}), 3.76(\mathrm{~s}, 3 \mathrm{H}), 3.38$ (d, $J=17.8 \mathrm{~Hz}, 1 \mathrm{H}), 3.14$ (d, $J=17.8 \mathrm{~Hz}, 1 \mathrm{H})$.

${ }^{13} \mathrm{C}$ NMR $\left(100 \mathrm{MHz}, \mathrm{CDCl}_{3}\right) \delta 169.3,163.7,159.7,151.1,136.0,135.9,135.1,132.9$, $130.9,129.8,129.1,129.0,128.8,128.6,128.2$, 128.1, 127.9, 126.1, 121.0, 114.8, 114.6, 113.8, 81.9, 55.3, 48.2, 46.9, 33.6.

HRMS (ESI) $m / z$ calculated for $\mathrm{C}_{29} \mathrm{H}_{26} \mathrm{NO}_{4}{ }^{+}[\mathrm{M}+\mathrm{H}]^{+}: 452.1856$, found 452.1847 .

HPLC analysis: (IA column, Hexane:2-propanol $=75: 25$, flow rate $=0.7 \mathrm{~mL} / \mathrm{min}$, wavelength $=254 \mathrm{~nm}$ ): $R \mathrm{t}=34.59$ (major), 24.31 (minor). 
(S,E)-2-benzyl-4-(3-methylbenzylidene)-9-phenyl-6-oxa-2-azaspiro[4.5]dec-8-ene1,7-dione (5ha)<smiles>Cc1cccc(/C=C2\CN(Cc3ccccc3)C(=O)C2(C)C2=CC(=O)OC2=O)c1</smiles>

White solid, $42.7 \mathrm{mg}, 98 \%$ yield, $\mathrm{mp}=185.4-185.9{ }^{\circ} \mathrm{C},[\alpha] \mathrm{D}^{25}=-1.3$ (c 0.420 , $\left.\mathrm{CH}_{2} \mathrm{Cl}_{2}\right), 96 \%$ ee.

${ }^{1} \mathrm{H}$ NMR (400 MHz, $\left.\mathrm{CDCl}_{3}\right) \delta 7.63-7.56(\mathrm{~m}, 2 \mathrm{H}), 7.52-7.43(\mathrm{~m}, 3 \mathrm{H}), 7.33(\mathrm{dt}, J=$ $11.8,6.7 \mathrm{~Hz}, 3 \mathrm{H}), 7.24(\mathrm{dd}, J=18.0,6.7 \mathrm{~Hz}, 3 \mathrm{H}), 7.10(\mathrm{~d}, J=7.4 \mathrm{~Hz}, 1 \mathrm{H}), 6.95$ (d, $J$ $=11.2 \mathrm{~Hz}, 2 \mathrm{H}), 6.87(\mathrm{~s}, 1 \mathrm{H}), 6.52(\mathrm{~s}, 1 \mathrm{H}), 4.64-4.50(\mathrm{~m}, 2 \mathrm{H}), 4.32-4.10(\mathrm{~m}, 2 \mathrm{H})$, $3.41(\mathrm{~d}, J=18.0 \mathrm{~Hz}, 1 \mathrm{H}), 3.14(\mathrm{~d}, J=18.0 \mathrm{~Hz}, 1 \mathrm{H}), 2.32$ (s, 3H).

${ }^{13} \mathrm{C}$ NMR $\left(100 \mathrm{MHz}, \mathrm{CDCl}_{3}\right) \delta 169.3,163.6,151.3,138.5,136.1,135.1,134.5,132.3$, $130.8,129.7,129.3,129.1,129.0,128.8,128.7,128.1,128.1,126.1,125.6,81.93$, $48.3,46.9,33.4,21.5$.

HRMS (ESI) $m / z$ calculated for $\mathrm{C}_{29} \mathrm{H}_{26} \mathrm{NO}_{3}{ }^{+}[\mathrm{M}+\mathrm{H}]^{+}: 436.1907$, found 436.1899 .

HPLC analysis: (IA column, Hexane:2-propanol $=75: 25$, flow rate $=0.7 \mathrm{~mL} / \mathrm{min}$, wavelength $=254 \mathrm{~nm}$ ): $R \mathrm{t}=25.23$ (major), 19.05 (minor).

(S,E)-2-benzyl-4-(4-fluorobenzylidene)-9-phenyl-6-oxa-2-azaspiro[4.5]dec-8-ene1,7-dione (5ia)<smiles>O=C1C=C(c2ccccc2)CC2(C/C(=C\c3ccc(F)cc3)CN2Cc2ccccc2)C1=O</smiles>

White solid, $43.1 \mathrm{mg}, 98 \%$ yield, $\mathrm{mp}=190.5-191.1{ }^{\circ} \mathrm{C},[\alpha] \mathrm{D}^{25}=-2.8$ (c 0.463 , $\left.\mathrm{CH}_{2} \mathrm{Cl}_{2}\right), 98 \%$ ee.

${ }^{1} \mathrm{H}$ NMR (400 MHz, $\left.\mathrm{CDCl}_{3}\right) \delta 7.57(\mathrm{~s}, 2 \mathrm{H}), 7.47(\mathrm{~s}, 3 \mathrm{H}), 7.38-7.23(\mathrm{~m}, 5 \mathrm{H}), 7.12(\mathrm{~s}$, $2 \mathrm{H}), 7.07-6.98(\mathrm{~m}, 2 \mathrm{H}), 6.87(\mathrm{~s}, 1 \mathrm{H}), 6.52(\mathrm{~s}, 1 \mathrm{H}), 4.56(\mathrm{~s}, 2 \mathrm{H}), 4.29-4.06(\mathrm{~m}, 2 \mathrm{H})$, $3.39(\mathrm{~d}, J=17.9 \mathrm{~Hz}, 1 \mathrm{H}), 3.14(\mathrm{~d}, J=17.9 \mathrm{~Hz}, 1 \mathrm{H})$.

${ }^{13} \mathrm{C}$ NMR (100 MHz, $\left.\mathrm{CDCl}_{3}\right) \delta 169.2,163.7,163.6,161.2,151.2,136.0,135.0,132.3$, $132.3,130.9,130.8,130.8,130.6,130.5,129.1,129.1,128.2,128.1,127.5,126.1$, 116.0, 115.8, 114.8, 81.8, 48.1, 47.0, 33.5. 
HRMS (ESI) $\mathrm{m} / \mathrm{z}$ calculated for $\mathrm{C}_{28} \mathrm{H}_{22} \mathrm{FNNaO}_{3}{ }^{+}[\mathrm{M}+\mathrm{Na}]^{+}: 462.1476$, found 462.1467.

HPLC analysis: (IA column, Hexane:2-propanol $=75: 25$, flow rate $=0.7 \mathrm{~mL} / \mathrm{min}$, wavelength $=254 \mathrm{~nm}$ ): $R \mathrm{t}=32.38$ (major), 26.41 (minor).

(S,E)-2-benzyl-9-phenyl-4-(4-(trifluoromethyl)benzylidene)-6-oxa-2-azaspiro[4.5] dec-8-ene-1,7-dione (5ja)<smiles>O=C1C=C(c2ccccc2)CC2(C=C(c3ccc(C(F)(F)F)cc3)CN(Cc3ccccc3)C2=O)O1</smiles>

White solid, $47.8 \mathrm{mg}, 98 \%$ yield, $\mathrm{mp}=145.1-146.7{ }^{\circ} \mathrm{C},[\alpha] \mathrm{D}^{25}=-2.7$ (c 0.489 , $\left.\mathrm{CH}_{2} \mathrm{Cl}_{2}\right), 95 \%$ ee.

${ }^{1} \mathrm{H}$ NMR $\left(400 \mathrm{MHz}, \mathrm{CDCl}_{3}\right) \delta 7.52(\mathrm{~d}, J=7.8 \mathrm{~Hz}, 4 \mathrm{H}), 7.39(\mathrm{~d}, J=4.9 \mathrm{~Hz}, 3 \mathrm{H}), 7.26$ $(\mathrm{d}, J=7.1 \mathrm{~Hz}, 3 \mathrm{H}), 7.18(\mathrm{~d}, J=7.2 \mathrm{~Hz}, 5 \mathrm{H}), 6.87$ (s, 1H), $6.45(\mathrm{~s}, 1 \mathrm{H}), 4.48(\mathrm{~s}, 2 \mathrm{H})$, 4.15 (d, $J=14.3 \mathrm{~Hz}, 1 \mathrm{H}), 4.07$ (d, $J=14.4 \mathrm{~Hz}, 1 \mathrm{H}), 3.31$ (d, $J=17.9 \mathrm{~Hz}, 1 \mathrm{H}), 3.07$ (d, $J=18.0 \mathrm{~Hz}, 1 \mathrm{H})$.

${ }^{13} \mathrm{C}$ NMR $\left(100 \mathrm{MHz}, \mathrm{CDCl}_{3}\right) \delta 169.0,163.4,150.9,138.0,135.9,135.4,134.9,130.9$, 129.1, 129.1, 128.9, 128.2, 127.2, 126.1, 125.8, 125.7, 125.7, 114.9, 81.6, 48.0, 47.0, 33.6.

HRMS (ESI) $m / z$ calculated for $\mathrm{C}_{29} \mathrm{H}_{23} \mathrm{~F}_{3} \mathrm{NO}_{3}{ }^{+}[\mathrm{M}+\mathrm{H}]^{+}: 490.1625$, found 490.1619 .

HPLC analysis: (IA column, Hexane:2-propanol $=75: 25$, flow rate $=0.8 \mathrm{~mL} / \mathrm{min}$, wavelength $=254 \mathrm{~nm}$ ): $R \mathrm{t}=25.33$ (major), 21.48 (minor).

(S,E)-2-benzyl-4-(4-chlorobenzylidene)-9-phenyl-6-oxa-2-azaspiro[4.5]dec-8-ene1,7-dione (5ka)<smiles>O=C1C=C(c2ccccc2)CC2(C/C(=C\c3ccc(Cl)cc3)CN2Cc2ccccc2)C1=O</smiles>

White solid, $44.4 \mathrm{mg}, 97 \%$ yield, $\mathrm{mp}=176.3-176.6{ }^{\circ} \mathrm{C},[\alpha] \mathrm{D}^{25}=1.1 \quad$ (c 0.460 , $\left.\mathrm{CH}_{2} \mathrm{Cl}_{2}\right), 98 \%$ ee. 
${ }^{1} \mathrm{H}$ NMR (400 MHz, Chloroform-d) $\delta 7.57$ (s, 2H), 7.46 (s, 3H), 7.32 (dd, J= 13.3, $7.9 \mathrm{~Hz}, 5 \mathrm{H}), 7.25(\mathrm{~s}, 2 \mathrm{H}), 7.07(\mathrm{~d}, J=7.9 \mathrm{~Hz}, 2 \mathrm{H}), 6.85(\mathrm{~s}, 1 \mathrm{H}), 6.51(\mathrm{~s}, 1 \mathrm{H}), 4.56(\mathrm{~s}$, 2H), $4.27-4.05$ (m, 2H), 3.38 (d, J=18.0 Hz, 1H), 3.14 (d, J=18.0 Hz, 1H).

${ }^{13} \mathrm{C}$ NMR $\left(100 \mathrm{MHz}, \mathrm{CDCl}_{3}\right) \delta 169.2,163.5,151.1,135.9,134.9,134.4,133.3,133.0$, $130.9,130.0,129.1,129.1,129.0,128.2,127.9,127.5,126.1,114.8,81.8,48.1,47.0$, 33.5 .

HRMS (ESI) $\mathrm{m} / \mathrm{z}$ calculated for $\mathrm{C}_{28} \mathrm{H}_{22} \mathrm{ClNNaO}_{3}{ }^{+}[\mathrm{M}+\mathrm{Na}]^{+}$: 478.1180, found 478.1173.

HPLC analysis: (IA column, Hexane:2-propanol $=75: 25$, flow rate $=0.7 \mathrm{~mL} / \mathrm{min}$, wavelength $=254 \mathrm{~nm}$ ): $R \mathrm{t}=34.01$ (major), 29.55 (minor).

(S,E)-2-benzyl-4-(4-bromobenzylidene)-9-phenyl-6-oxa-2-azaspiro[4.5]dec-8-ene1,7-dione (5la)<smiles>O=C1C=C(c2ccccc2)CC2(C/C(=C\c3ccc(Br)cc3)CN2Cc2ccccc2)C1=O</smiles>

White solid, $49.3 \mathrm{mg}, 99 \%$ yield, $\mathrm{mp}=204.3-205.1{ }^{\circ} \mathrm{C},[\alpha] \mathrm{D}^{25}=-2.9$ (c 0.483 , $\left.\mathrm{CH}_{2} \mathrm{Cl}_{2}\right), 96 \%$ ee.

${ }^{1} \mathrm{H}$ NMR (400 MHz, $\left.\mathrm{CDCl}_{3}\right) \delta 7.50(\mathrm{~s}, 2 \mathrm{H}), 7.39$ (s, 5H), $7.32-7.09(\mathrm{~m}, 6 \mathrm{H}), 6.93$ (d, $J=6.7 \mathrm{~Hz}, 2 \mathrm{H}), 6.76(\mathrm{~s}, 1 \mathrm{H}), 6.44(\mathrm{~s}, 1 \mathrm{H}), 4.48(\mathrm{~s}, 2 \mathrm{H}), 4.12(\mathrm{~d}, J=13.1 \mathrm{~Hz}, 1 \mathrm{H})$, $4.03(\mathrm{~d}, J=13.1 \mathrm{~Hz}, 1 \mathrm{H}), 3.30(\mathrm{~d}, J=17.4 \mathrm{~Hz}, 1 \mathrm{H}), 3.06(\mathrm{~d}, J=17.5 \mathrm{~Hz}, 1 \mathrm{H})$.

${ }^{13} \mathrm{C}$ NMR $\left(100 \mathrm{MHz}, \mathrm{CDCl}_{3}\right) \delta 169.1,163.5,151.0,136.0,134.9,133.5,133.5,132.0$, $130.9,130.2,129.1,129.1,128.2,127.5,126.1,122.6,114.9,81.8,48.2$, 47.0, 33.6.

HRMS (ESI) $\mathrm{m} / \mathrm{z}$ calculated for $\mathrm{C}_{28} \mathrm{H}_{22} \mathrm{BrNNaO}_{3}{ }^{+}[\mathrm{M}+\mathrm{Na}]^{+}$: 522.0675 , found 522.0669 .

HPLC analysis: (IA column, Hexane:2-propanol $=70: 30$, flow rate $=1.0 \mathrm{~mL} / \mathrm{min}$, wavelength $=254 \mathrm{~nm}$ ): $R \mathrm{t}=37.20$ (major), 28.76 (minor).

(S,E)-2-benzyl-4-(4-methoxybenzylidene)-9-phenyl-6-oxa-2-azaspiro[4.5]dec-8-en e-1,7-dione (5ma) 
<smiles>COc1ccc(/C=C2\CN(Cc3ccccc3)C(=O)C23CC(c2ccccc2)=CC(=O)O3)cc1</smiles>

White solid, $44.1 \mathrm{mg}, 98 \%$ yield, $\mathrm{mp}=150.6-151.6{ }^{\circ} \mathrm{C},[\alpha] \mathrm{D}^{25}=4.7$ (c 0.451 , $\mathrm{CH}_{2} \mathrm{Cl}_{2}$ ), $>99 \%$ ee.

${ }^{1} \mathrm{H}$ NMR $\left(400 \mathrm{MHz}, \mathrm{CDCl}_{3}\right) \delta 7.51(\mathrm{~s}, 2 \mathrm{H}), 7.39(\mathrm{~s}, 3 \mathrm{H}), 7.34-7.10(\mathrm{~m}, 8 \mathrm{H}), 7.01(\mathrm{~d}$, $J=8.0 \mathrm{~Hz}, 2 \mathrm{H}), 6.88-6.66(\mathrm{~m}, 4 \mathrm{H}), 6.44(\mathrm{~s}, 1 \mathrm{H}), 4.49(\mathrm{~s}, 2 \mathrm{H}), 4.18(\mathrm{~d}, J=13.6 \mathrm{~Hz}$, $1 \mathrm{H}), 4.04(\mathrm{~d}, J=13.6 \mathrm{~Hz}, 1 \mathrm{H}), 3.72(\mathrm{~s}, 2 \mathrm{H}), 3.31$ (d, $J=17.9 \mathrm{~Hz}, 1 \mathrm{H}), 3.06(\mathrm{~d}, J=$ $18.0 \mathrm{~Hz}, 1 \mathrm{H})$.

${ }^{13} \mathrm{C}$ NMR $\left(100 \mathrm{MHz}, \mathrm{CDCl}_{3}\right) \delta 169.4,163.7,159.6,151.4,136.2,136.2,135.1,130.2$, $130.2,130.0,129.1,129.0,128.3,128.2,128.1,127.3,126.1,114.9,114.2,82.1,55.3$, 48.4, 47.0, 33.4.

HRMS (ESI) $m / z$ calculated for $\mathrm{C}_{29} \mathrm{H}_{25} \mathrm{NNaO}_{4}{ }^{+}[\mathrm{M}+\mathrm{Na}]^{+}$: 474.1676, found 474.1665.

HPLC analysis: (IA column, Hexane:2-propanol $=75: 25$, flow rate $=0.8 \mathrm{~mL} / \mathrm{min}$, wavelength $=254 \mathrm{~nm}$ ): $R \mathrm{t}=36.51$ (major), 25.92 (minor).

(S,E)-2-benzyl-4-(2,4-dichlorobenzylidene)-9-phenyl-6-oxa-2-azaspiro[4.5]dec-8-e ne-1,7-dione (5na)<smiles>O=C1C=C(c2ccccc2)CC2(C/C(=C\c3ccc(Cl)cc3Cl)CN(Cc3ccccc3)C2=O)O1</smiles>

White solid, $44.1 \mathrm{mg}, 90 \%$ yield, $\mathrm{mp}=164.5-164.9{ }^{\circ} \mathrm{C},[\alpha] \mathrm{D}^{25}=15.2$ (c 0.451 , $\mathrm{CH}_{2} \mathrm{Cl}_{2}$ ), 90\% ee.

${ }^{1} \mathrm{H}$ NMR (400 MHz, $\left.\mathrm{CDCl}_{3}\right) \delta 7.64-7.56(\mathrm{~m}, 2 \mathrm{H}), 7.47(\mathrm{~d}, J=4.9 \mathrm{~Hz}, 3 \mathrm{H}), 7.40-$ $7.30(\mathrm{~m}, 4 \mathrm{H}), 7.24(\mathrm{~s}, 2 \mathrm{H}), 7.23-7.19(\mathrm{~m}, 1 \mathrm{H}), 7.09(\mathrm{~s}, 1 \mathrm{H}), 7.04(\mathrm{~d}, J=8.3 \mathrm{~Hz}, 1 \mathrm{H})$, $6.50(\mathrm{~s}, 1 \mathrm{H}), 4.68-4.47(\mathrm{~m}, 2 \mathrm{H}), 4.19(\mathrm{~d}, J=14.3 \mathrm{~Hz}, 1 \mathrm{H}), 3.92(\mathrm{~d}, J=15.7 \mathrm{~Hz}, 1 \mathrm{H})$, $3.48(\mathrm{~d}, J=18.0 \mathrm{~Hz}, 1 \mathrm{H}), 3.16(\mathrm{~d}, J=18.0 \mathrm{~Hz}, 1 \mathrm{H})$.

${ }^{13} \mathrm{C}$ NMR $\left(100 \mathrm{MHz}, \mathrm{CDCl}_{3}\right) \delta 168.9,163.1,152.1,136.0,135.6,135.0,134.8,134.7$, $131.2,131.0,129.8,129.8,129.1,129.1,128.2,127.3,126.2,124.6,114.8,81.7,47.7$, 47.0, 32.6 . 
HRMS (ESI) $\mathrm{m} / \mathrm{z}$ calculated for $\mathrm{C}_{28} \mathrm{H}_{21} \mathrm{Cl}_{2} \mathrm{NNaO}_{3}{ }^{+}[\mathrm{M}+\mathrm{Na}]^{+}$: 512.0791, found 512.0783 .

HPLC analysis: (IA column, Hexane:2-propanol $=75: 25$, flow rate $=0.7 \mathrm{~mL} / \mathrm{min}$, wavelength $=254 \mathrm{~nm}$ ): $R \mathrm{t}=23.40$ (major), 20.46 (minor).

(S,E)-2-benzyl-4-(4-fluoro-2-methylbenzylidene)-9-phenyl-6-oxa-2-azaspiro[4.5]d ec-8-ene-1,7-dione (50a)<smiles>Cc1cc(F)ccc1/C=C1\CN(Cc2ccccc2)C(=O)[C@]12CC(=O)C=C(c1ccccc1)O2</smiles>

White solid, $44.5 \mathrm{mg}, 98 \%$ yield, $\mathrm{mp}=172.8-173.7{ }^{\circ} \mathrm{C},[\alpha] \mathrm{D}^{25}=-3.2$ (c 0.463 , $\mathrm{CH}_{2} \mathrm{Cl}_{2}$ ), $97 \%$ ee.

${ }^{1} \mathrm{H}$ NMR $\left(400 \mathrm{MHz}, \mathrm{CDCl}_{3}\right) \delta 7.58(\mathrm{~s}, 2 \mathrm{H}), 7.48(\mathrm{~s}, 3 \mathrm{H}), 7.38-7.29(\mathrm{~m}, 3 \mathrm{H}), 7.25(\mathrm{~d}$, $J=5.7 \mathrm{~Hz}, 2 \mathrm{H}), 6.96(\mathrm{~s}, 2 \mathrm{H}), 6.91-6.79(\mathrm{~m}, 2 \mathrm{H}), 6.52(\mathrm{~s}, 1 \mathrm{H}), 4.62-4.45(\mathrm{~m}, 2 \mathrm{H})$, $4.18-3.89(\mathrm{~m}, 2 \mathrm{H}), 3.45$ (d, $J=17.9 \mathrm{~Hz}, 1 \mathrm{H}), 3.13(\mathrm{~d}, J=17.9 \mathrm{~Hz}, 1 \mathrm{H}), 2.18(\mathrm{~s}, 3 \mathrm{H})$.

${ }^{13} \mathrm{C} \mathrm{NMR}\left(100 \mathrm{MHz}, \mathrm{CDCl}_{3}\right) \delta 169.2,163.6,163.5,161.2,151.5,139.5,139.4,135.0$, $136.01,133.4,130.9,129.4,129.4,129.3,129.2,129.1,129.0,128.2,128.1,126.1$, $117.5,117.3,114.9,113.0,112.8,81.7,47.7,47.0,33.1,19.9$.

HRMS (ESI) $\mathrm{m} / \mathrm{z}$ calculated for $\mathrm{C}_{29} \mathrm{H}_{24} \mathrm{FNNaO}_{3}{ }^{+}[\mathrm{M}+\mathrm{Na}]^{+}: 476.1632$, found 476.1626 .

HPLC analysis: (IA column, Hexane:2-propanol $=75: 25$, flow rate $=0.7 \mathrm{~mL} / \mathrm{min}$, wavelength $=254 \mathrm{~nm}$ ): $R \mathrm{t}=23.17$ (major), 19.37 (minor).

(S,E)-2-benzyl-4-(naphthalen-2-ylmethylene)-9-phenyl-6-oxa-2-azaspiro[4.5]dec8-ene-1,7-dione (5pa)<smiles>O=C1C=C(c2ccccc2)CC2(C=C(c3ccc4ccccc4c3)CN2Cc2ccccc2)C1=O</smiles>

White solid, $46.7 \mathrm{mg}, 99 \%$ yield, $\mathrm{mp}=184.4-185.3{ }^{\circ} \mathrm{C},[\alpha] \mathrm{D}^{25}=6.5$ (c 0.482 , $\mathrm{CH}_{2} \mathrm{Cl}_{2}$ ), $98 \%$ ee. 
${ }^{1} \mathrm{H}$ NMR (400 MHz, $\left.\mathrm{CDCl}_{3}\right) \delta 7.78(\mathrm{~d}, J=7.6 \mathrm{~Hz}, 3 \mathrm{H}), 7.59(\mathrm{t}, J=4.7 \mathrm{~Hz}, 3 \mathrm{H}), 7.47$ $(\mathrm{dt}, J=5.5,3.1 \mathrm{~Hz}, 5 \mathrm{H}), 7.36-7.23(\mathrm{~m}, 7 \mathrm{H}), 7.05(\mathrm{~s}, 1 \mathrm{H}), 6.54(\mathrm{~s}, 1 \mathrm{H}), 4.57(\mathrm{~s}, 2 \mathrm{H})$, $4.39-4.20(\mathrm{~m}, 2 \mathrm{H}), 3.43$ (d, $J=18.0 \mathrm{~Hz}, 1 \mathrm{H}), 3.19$ (d, $J=18.0 \mathrm{~Hz}, 1 \mathrm{H})$.

${ }^{13} \mathrm{C}$ NMR $\left(100 \mathrm{MHz}, \mathrm{CDCl}_{3}\right) \delta 169.3,163.6,151.2,136.1,135.1,133.1,132.9,132.9$, $132.1,130.9,129.1,129.0,128.8,128.5,128.5,128.2,128.2,128.1,127.7,126.9$, $126.7,126.1,125.9,114.9,82.0,48.4,47.0,33.5$.

HRMS (ESI) $m / z$ calculated for $\mathrm{C}_{32} \mathrm{H}_{25} \mathrm{NNaO}_{3}{ }^{+}[\mathrm{M}+\mathrm{Na}]^{+}$: 494.1727, found 494.1720.

HPLC analysis: (IA column, Hexane:2-propanol $=75: 25$, flow rate $=0.7 \mathrm{~mL} / \mathrm{min}$, wavelength $=254 \mathrm{~nm}$ ): $R \mathrm{t}=34.20$ (major), 27.53 (minor).

(S,E)-2-benzyl-9-phenyl-4-(thiophen-2-ylmethylene)-6-oxa-2-azaspiro[4.5]dec-8-e ne-1,7-dione (5qa)<smiles>O=C1C=C(c2ccccc2)CC2(C/C(=C\c3cccs3)CN2Cc2ccccc2)C1=O</smiles>

White solid, $41.9 \mathrm{mg}, 98 \%$ yield, $\mathrm{mp}=178.1-178.6{ }^{\circ} \mathrm{C},[\alpha] \mathrm{D}^{25}=8.3($ c 0.405 , $\left.\mathrm{CH}_{2} \mathrm{Cl}_{2}\right), 96 \%$ ee.

${ }^{1} \mathrm{H}$ NMR (400 MHz, $\left.\mathrm{CDCl}_{3}\right) \delta 7.57(\mathrm{dd}, J=6.5,3.0 \mathrm{~Hz}, 2 \mathrm{H}), 7.45(\mathrm{dd}, J=5.1,1.7 \mathrm{~Hz}$, $3 \mathrm{H}), 7.39-7.25(\mathrm{~m}, 6 \mathrm{H}), 7.11(\mathrm{~s}, 1 \mathrm{H}), 7.03(\mathrm{dd}, J=5.0,3.7 \mathrm{~Hz}, 1 \mathrm{H}), 6.99$ (d, J= 3.2 $\mathrm{Hz}, 1 \mathrm{H}), 6.52$ (s, 1H), 4.59 (s, 2H), $4.28-4.08$ (m, 2H), 3.35 (d, J = 18.1 Hz, 1H), $3.14(\mathrm{~d}, J=19.7 \mathrm{~Hz}, 1 \mathrm{H})$.

${ }^{13} \mathrm{C}$ NMR $\left(100 \mathrm{MHz}, \mathrm{CDCl}_{3}\right) \delta 169.4,163.6,151.1,138.1,136.0,135.0,130.8,130.1$, 129.3, 129.1, 129.1, 128.3, 128.1, 127.9, 127.7, 126.1, 121.7, 114.8, 81.7, 48.4, 47.0, 33.9 .

HRMS (ESI) $m / z$ calculated for $\mathrm{C}_{26} \mathrm{H}_{21} \mathrm{NNaO}_{3} \mathrm{~S}^{+}[\mathrm{M}+\mathrm{Na}]^{+}: 450.1134$, found 450.1129 .

HPLC analysis: (IA column, Hexane:2-propanol $=75: 25$, flow rate $=0.7 \mathrm{~mL} / \mathrm{min}$, wavelength $=254 \mathrm{~nm}$ ): $R \mathrm{t}=40.39$ (major), 26.29 (minor).

(S,E)-2-benzyl-9-phenyl-4-((E)-3-phenylallylidene)-6-oxa-2-azaspiro[4.5]dec-8-en e-1,7-dione (5ra) 


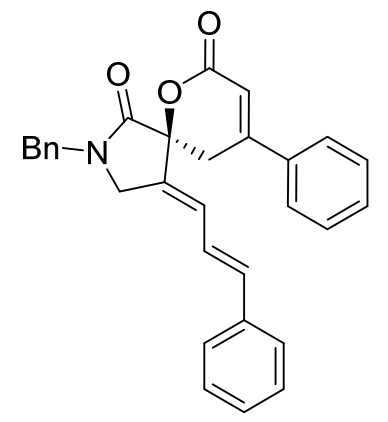

White solid, $43.3 \mathrm{mg}, 97 \%$ yield, $\mathrm{mp}=190.9-191.6{ }^{\circ} \mathrm{C},[\alpha] \mathrm{D}^{25}=36.1$ (c 0.420 , $\left.\mathrm{CH}_{2} \mathrm{Cl}_{2}\right), 97 \%$ ee.

${ }^{1} \mathrm{H}$ NMR $\left(400 \mathrm{MHz}, \mathrm{CDCl}_{3}\right) \delta 7.61-7.56(\mathrm{~m}, 2 \mathrm{H}), 7.47(\mathrm{dd}, J=5.1,1.8 \mathrm{~Hz}, 3 \mathrm{H})$, $7.40-7.34(\mathrm{~m}, 4 \mathrm{H}), 7.34-7.23(\mathrm{~m}, 6 \mathrm{H}), 6.67-6.56(\mathrm{~m}, 3 \mathrm{H}), 6.52(\mathrm{t}, J=1.4 \mathrm{~Hz}, 1 \mathrm{H})$, $4.68-4.53(\mathrm{~m}, 2 \mathrm{H}), 4.18(\mathrm{~d}, J=14.1 \mathrm{~Hz}, 1 \mathrm{H}), 4.02(\mathrm{~d}, J=13.2 \mathrm{~Hz}, 1 \mathrm{H}), 3.38$ (dd, $J$ $=18.0,1.5 \mathrm{~Hz}, 1 \mathrm{H}), 3.08(\mathrm{dd}, J=18.0,1.5 \mathrm{~Hz}, 1 \mathrm{H})$.

${ }^{13} \mathrm{C} \mathrm{NMR}\left(100 \mathrm{MHz}, \mathrm{CDCl}_{3}\right) \delta 169.6,163.5,151.5,137.2,136.2,135.0,132.0,130.8$, 129.1, 129.1, 128.8, 128.7, 128.3, 128.2, 127.9, 126.8, 126.1, 122.3, 114.8, 81.3, 47.0, 33.1 .

HRMS (ESI) $m / z$ calculated for $\mathrm{C}_{30} \mathrm{H}_{25} \mathrm{NNaO}_{3}{ }^{+}[\mathrm{M}+\mathrm{Na}]^{+}:$470.1727, found 470.1720.

HPLC analysis: (IA column, Hexane:2-propanol $=75: 25$, flow rate $=0.7 \mathrm{~mL} / \mathrm{min}$, wavelength $=254 \mathrm{~nm}$ ): $R \mathrm{t}=28.59$ (major), 25.87 (minor).

(S,E)-methyl (E)-6-oxo-4-phenyl-2-styryl-3,6-dihydro-2H-pyran-2-carboxylate (5ta)<smiles>COC(=O)C1(/C=C/c2ccccc2)CC(c2ccccc2)=CC(=O)O1</smiles>

White solid, $26.4 \mathrm{mg}, 79 \%$ yield, $\mathrm{mp}=174.7-175.3{ }^{\circ} \mathrm{C},[\alpha] \mathrm{D}^{25}=-66.0$ (c 0.264 , $\left.\mathrm{CH}_{2} \mathrm{Cl}_{2}\right), 88 \%$ ee.

${ }^{1} \mathrm{H}$ NMR $\left(400 \mathrm{MHz}, \mathrm{CDCl}_{3}\right) \delta 7.59-7.54(\mathrm{~m}, 2 \mathrm{H}), 7.49-7.41(\mathrm{~m}, 5 \mathrm{H}), 7.39-7.28$ (m, 3H), $7.02(\mathrm{~d}, \mathrm{~J}=16.1 \mathrm{~Hz}, 1 \mathrm{H}), 6.41-6.35(\mathrm{~m}, 2 \mathrm{H}), 3.77$ (s, 3H), 3.65 (d, J = 17.5 $\mathrm{Hz}, 1 \mathrm{H}), 3.04$ (dd, J = 17.5, $2.3 \mathrm{~Hz}, 1 \mathrm{H})$.

${ }^{13} \mathrm{C}$ NMR $\left(100 \mathrm{MHz}, \mathrm{CDCl}_{3}\right) \delta 170.8,163.7,153.3,135.6,135.3,132.6,131.0,129.1$, $128.8,128.7,126.9,126.3,124.8,115.2,83.1,53.6,35.2$.

HPLC analysis: (AD-H column, Hexane:2-propanol $=90: 10$, flow rate $=0.9 \mathrm{~mL} / \mathrm{min}$, wavelength $=254 \mathrm{~nm}$ ): $R \mathrm{t}=40.73$ (major), 21.58 (minor). 
(S,E)-2-benzyl-4-benzylidene-9-(m-tolyl)-6-oxa-2-azaspiro[4.5]dec-8-ene-1,7-dion e (5ad)<smiles>Cc1cccc(C2=CC(=O)OC3(C=C(c4ccccc4)CN3Cc3ccccc3)C2=O)c1</smiles>

White solid, $42.7 \mathrm{mg}, 98 \%$ yield, $\mathrm{mp}=164.8-166.1{ }^{\circ} \mathrm{C},[\alpha] \mathrm{D}^{25}=1.4$ (c 0.435 , $\left.\mathrm{CH}_{2} \mathrm{Cl}_{2}\right), 97 \%$ ee.

${ }^{1} \mathrm{H}$ NMR (400 MHz, $\left.\mathrm{CDCl}_{3}\right) \delta 7.34-7.22(\mathrm{~m}, 8 \mathrm{H}), 7.22-7.13(\mathrm{~m}, 5 \mathrm{H}), 7.06(\mathrm{~d}, J=$ $7.3 \mathrm{~Hz}, 2 \mathrm{H}), 6.82(\mathrm{~s}, 1 \mathrm{H}), 6.42(\mathrm{~s}, 1 \mathrm{H}), 4.48(\mathrm{~s}, 2 \mathrm{H}), 4.18(\mathrm{~d}, J=14.3 \mathrm{~Hz}, 1 \mathrm{H}), 4.07$ (d, $J=14.2 \mathrm{~Hz}, 1 \mathrm{H}), 3.31(\mathrm{~d}, J=18.0 \mathrm{~Hz}, 1 \mathrm{H}), 3.05(\mathrm{~d}, J=18.0 \mathrm{~Hz}, 1 \mathrm{H}), 2.32(\mathrm{~s}, 3 \mathrm{H})$.

${ }^{13} \mathrm{C} \mathrm{NMR}\left(100 \mathrm{MHz}, \mathrm{CDCl}_{3}\right) \delta 169.3,163.7,151.4,138.8,136.1,135.1,134.6,132.6$, $131.6,129.0,129.0,128.8,128.7,128.6,128.5,128.2,128.1,126.8,123.3,114.7$, $81.9,48.3,47.0,33.5,21.5$.

HRMS (ESI) $m / z$ calculated for $\mathrm{C}_{29} \mathrm{H}_{25} \mathrm{NNaO}_{3}{ }^{+}[\mathrm{M}+\mathrm{Na}]^{+}:$458.1727, found 458.1722.

HPLC analysis: (IA column, Hexane:2-propanol $=75: 25$, flow rate $=0.8 \mathrm{~mL} / \mathrm{min}$, wavelength $=254 \mathrm{~nm}$ ): $R \mathrm{t}=17.92$ (major), 14.72 (minor).

(S,E)-2-benzyl-4-benzylidene-9-(3-methoxyphenyl)-6-oxa-2-azaspiro[4.5]dec-8-en e-1,7-dione (5ae)<smiles>COc1cccc(C2=CC(=O)OC3(C2)C/C(=C\c2ccccc2)CN(Cc2ccccc2)C3=O)c1</smiles>

White solid, $44.0 \mathrm{mg}, 98 \%$ yield, $\mathrm{mp}=137.4-138.4{ }^{\circ} \mathrm{C},[\alpha] \mathrm{D}^{25}=1.3($ c 0.452 , $\mathrm{CH}_{2} \mathrm{Cl}_{2}$ ), $98 \%$ ee.

${ }^{1} \mathrm{H}$ NMR (400 MHz, $\left.\mathrm{CDCl}_{3}\right) \delta 7.54-7.25(\mathrm{~m}, 10 \mathrm{H}), 7.17$ (d, J=7.1 Hz, 4H), 7.03 $(\mathrm{dd}, J=8.2,2.2 \mathrm{~Hz}, 1 \mathrm{H}), 6.92(\mathrm{~s}, 1 \mathrm{H}), 6.54(\mathrm{~s}, 1 \mathrm{H}), 4.59$ (s, 2H), 4.29 (d, J=13.8 Hz, $1 \mathrm{H}), 4.18(\mathrm{~d}, J=14.1 \mathrm{~Hz}, 1 \mathrm{H}), 3.87$ (s, 3H), 3.40 (d, $J=17.9 \mathrm{~Hz}, 1 \mathrm{H}), 3.16(\mathrm{~d}, J=$ $17.9 \mathrm{~Hz}, 1 \mathrm{H})$.

${ }^{13} \mathrm{C}$ NMR $\left(100 \mathrm{MHz}, \mathrm{CDCl}_{3}\right) \delta 169.3,163.6,160.0,151.1,137.5,135.0,134.6,132.5$, $130.2,129.1,128.8,128.7,128.7,128.5,128.2,128.1,118.5,116.3,115.1,111.7$, $81.9,55.5,48.3,47.0,33.6$.

HRMS (ESI) $m / z$ calculated for $\mathrm{C}_{29} \mathrm{H}_{25} \mathrm{NNaO}_{4}{ }^{+}[\mathrm{M}+\mathrm{Na}]^{+}: 474.1676$, found 474.1672. 
HPLC analysis: (IA column, Hexane:2-propanol $=75: 25$, flow rate $=0.8 \mathrm{~mL} / \mathrm{min}$, wavelength $=254 \mathrm{~nm}$ ): $R \mathrm{t}=27.64$ (major), 21.85 (minor).

(S,E)-2-benzyl-4-benzylidene-9-(3-chlorophenyl)-6-oxa-2-azaspiro[4.5]dec-8-ene1,7-dione (5af)<smiles>O=C1C=C(c2cccc(Cl)c2)CC2(C=C(c3ccccc3)CN2Cc2ccccc2)C1=O</smiles>

White solid, $44.2 \mathrm{mg}, 97 \%$ yield, $\mathrm{mp}=187.5-188.9{ }^{\circ} \mathrm{C},[\alpha] \mathrm{D}^{25}=-2.8$ (c 0.456 , $\left.\mathrm{CH}_{2} \mathrm{Cl}_{2}\right), 99 \%$ ee.

${ }^{1} \mathrm{H}$ NMR $\left(400 \mathrm{MHz}, \mathrm{CDCl}_{3}\right) \delta 7.34(\mathrm{~m}, 14 \mathrm{H}), 6.93(\mathrm{~s}, 1 \mathrm{H}), 6.54(\mathrm{~s}, 1 \mathrm{H}), 4.58(\mathrm{~s}, 2 \mathrm{H})$, 4.24 (q, $J=13.7 \mathrm{~Hz}, 2 \mathrm{H}), 3.32$ (d, $J=17.7 \mathrm{~Hz}, 1 \mathrm{H}), 3.17$ (d, $J=17.6 \mathrm{~Hz}, 1 \mathrm{H})$.

${ }^{13} \mathrm{C}$ NMR $\left(100 \mathrm{MHz}, \mathrm{CDCl}_{3}\right) \delta 169.2,163.3,149.4,137.9,135.2,135.0,134.5,132.2$, $130.7,130.4,129.1,128.9,128.8,128.7,128.6,128.2,128.2,126.3,124.3,116.0$, $81.8,48.3,47.0,33.7$.

HRMS (ESI) $\mathrm{m} / \mathrm{z}$ calculated for $\mathrm{C}_{28} \mathrm{H}_{22} \mathrm{ClNNaO}_{3}{ }^{+}[\mathrm{M}+\mathrm{Na}]^{+}$: 478.1180, found 478.1180 .

HPLC analysis: (IB column, Hexane:2-propanol $=80: 20$, flow rate $=1.0 \mathrm{~mL} / \mathrm{min}$, wavelength $=254 \mathrm{~nm}$ ): $R \mathrm{t}=37.44$ (major), 25.37 (minor).

(S,E)-2-benzyl-4-benzylidene-9-(4-fluorophenyl)-6-oxa-2-azaspiro[4.5]dec-8-ene1,7-dione (5ag)<smiles>O=C1C=C(c2ccc(F)cc2)CC2(C1)C(=O)N(Cc1ccccc1)CC2=Cc1ccccc1</smiles>

White solid, $41.8 \mathrm{mg}, 95 \%$ yield, $\mathrm{mp}=105.3-106.8{ }^{\circ} \mathrm{C},[\alpha] \mathrm{D}^{25}=-5.0$ (c 0.439 , $\left.\mathrm{CH}_{2} \mathrm{Cl}_{2}\right), 98 \%$ ee.

${ }^{1} \mathrm{H}$ NMR (400 MHz, $\left.\mathrm{CDCl}_{3}\right) \delta 7.59-7.43(\mathrm{~m}, 2 \mathrm{H}), 7.33-7.15(\mathrm{~m}, 8 \mathrm{H}), 7.08(\mathrm{~d}, J=$ $5.8 \mathrm{~Hz}, 4 \mathrm{H}), 6.83(\mathrm{~s}, 1 \mathrm{H}), 6.41(\mathrm{~s}, 1 \mathrm{H}), 4.49(\mathrm{~s}, 2 \mathrm{H}), 4.26-4.14(\mathrm{~m}, 1 \mathrm{H}), 4.08(\mathrm{~d}, J=$ $15.5 \mathrm{~Hz}, 1 \mathrm{H}), 3.26(\mathrm{~d}, J=17.9 \mathrm{~Hz}, 1 \mathrm{H}), 3.06(\mathrm{~d}, J=17.9 \mathrm{~Hz}, 1 \mathrm{H})$.

${ }^{13} \mathrm{C} \mathrm{NMR}\left(100 \mathrm{MHz}, \mathrm{CDCl}_{3}\right) \delta 169.2,165.5,163.4,163.0,149.9,135.0,134.6,132.4$, $132.2,132.2,129.0,128.8,128.7,128.5,128.2,128.2,128.1,128.1,116.4,116.2$, $114.8,114.8,81.8,48.3,47.0,33.6$. 
HRMS (ESI) $\mathrm{m} / \mathrm{z}$ calculated for $\mathrm{C}_{28} \mathrm{H}_{22} \mathrm{FNNaO}_{3}{ }^{+}[\mathrm{M}+\mathrm{Na}]^{+}: 462.1476$, found 462.1474 .

HPLC analysis: (IA column, Hexane:2-propanol $=75: 25$, flow rate $=0.8 \mathrm{~mL} / \mathrm{min}$, wavelength $=254 \mathrm{~nm}$ ): $R \mathrm{t}=31.73$ (major), 22.58 (minor).

(S,E)-2-benzyl-4-benzylidene-9-(4-chlorophenyl)-6-oxa-2-azaspiro[4.5]dec-8-ene1,7-dione (5ah)<smiles>O=C1C=C(c2ccc(Cl)cc2)CC2(C1)C(=O)N(Cc1ccccc1)CC2=Cc1ccccc1</smiles>

White solid, $41.0 \mathrm{mg}, 90 \%$ yield, $\mathrm{mp}=138.1-138.7{ }^{\circ} \mathrm{C},[\alpha] \mathrm{D}^{25}=10.4$ (c 0.410 , $\left.\mathrm{CH}_{2} \mathrm{Cl}_{2}\right), 97 \%$ ee.

${ }^{1} \mathrm{H}$ NMR (400 MHz, $\left.\mathrm{CDCl}_{3}\right) \delta 7.53(\mathrm{~d}, J=8.1 \mathrm{~Hz}, 2 \mathrm{H}), 7.44$ (d, $\left.J=7.8 \mathrm{~Hz}, 2 \mathrm{H}\right), 7.42$ -7.25 (m, 9H), 7.18 (d, J=7.4 Hz, 2H), 6.92 (s, 1H), 6.52 (s, 1H), 4.57 (s, 2H), 4.24 (q, $J=14.0 \mathrm{~Hz}, 2 \mathrm{H}), 3.34$ (d, $J=17.8 \mathrm{~Hz}, 1 \mathrm{H}), 3.17$ (d, $J=17.7 \mathrm{~Hz}, 1 \mathrm{H})$.

${ }^{13} \mathrm{C}$ NMR $\left(100 \mathrm{MHz}, \mathrm{CDCl}_{3}\right) \delta 169.3,163.5,149.7,136.9,135.0,134.6,134.4,132.3$, 129.4, 129.1, 128.8, 128.8, 128.6, 128.2, 128.1, 127.5, 115.2, 81.8, 48.3, 47.0, 33.6.

HRMS (ESI) $\mathrm{m} / \mathrm{z}$ calculated for $\mathrm{C}_{28} \mathrm{H}_{22} \mathrm{ClNNaO}_{3}{ }^{+}[\mathrm{M}+\mathrm{Na}]^{+}: 478.1180$, found 478.1175 .

HPLC analysis: (IA column, Hexane:2-propanol $=75: 25$, flow rate $=0.8 \mathrm{~mL} / \mathrm{min}$, wavelength $=254 \mathrm{~nm}$ ): $R \mathrm{t}=31.11$ (major), 23.99 (minor).

(S,E)-2-benzyl-4-benzylidene-9-methyl-6-oxa-2-azaspiro[4.5]dec-8-ene-1,7-dionee (5ai)<smiles>CC1=CC(=O)C(=Cc2ccccc2)CN(Cc2ccccc2)C1=O</smiles>

White solid, $27.8 \mathrm{mg}, 77 \%$ yield, $\mathrm{mp}=116.3-117.9{ }^{\circ} \mathrm{C},[\alpha] \mathrm{D}^{25}=-0.9$ (c 0.249 , $\left.\mathrm{CH}_{2} \mathrm{Cl}_{2}\right), 97 \%$ ee.

${ }^{1} \mathrm{H}$ NMR (400 MHz, $\left.\mathrm{CDCl}_{3}\right) \delta 7.33(\mathrm{dt}, J=19.4,7.0 \mathrm{~Hz}, 6 \mathrm{H}), 7.24(\mathrm{~s}, 2 \mathrm{H}), 7.15(\mathrm{~d}, J$ $=7.2 \mathrm{~Hz}, 2 \mathrm{H}), 6.82(\mathrm{~s}, 1 \mathrm{H}), 6.00(\mathrm{~s}, 1 \mathrm{H}), 4.55(\mathrm{~s}, 2 \mathrm{H}), 4.26-4.06(\mathrm{~m}, 2 \mathrm{H}), 2.90-$ $2.66(\mathrm{~m}, 2 \mathrm{H}), 2.06(\mathrm{~s}, 3 \mathrm{H})$. 
${ }^{13} \mathrm{C} \mathrm{NMR}\left(100 \mathrm{MHz}, \mathrm{CDCl}_{3}\right) \delta 169.5,163.1,153.4,135.1,134.7,132.6,129.0,128.8$, $128.7,128.4,128.1,128.0,116.5,81.7,48.2,46.9,36.3,23.1$.

HRMS (ESI) $m / z$ calculated for $\mathrm{C}_{23} \mathrm{H}_{21} \mathrm{NNaO}_{3}{ }^{+}[\mathrm{M}+\mathrm{Na}]^{+}: 382.1414$, found 382.1415 .

HPLC analysis: (IA column, Hexane:2-propanol $=75: 25$, flow rate $=0.8 \mathrm{~mL} / \mathrm{min}$, wavelength $=254 \mathrm{~nm}$ ): $R \mathrm{t}=14.39$ (major), 18.47 (minor).

\subsection{Gram-scale of $5 a a$}

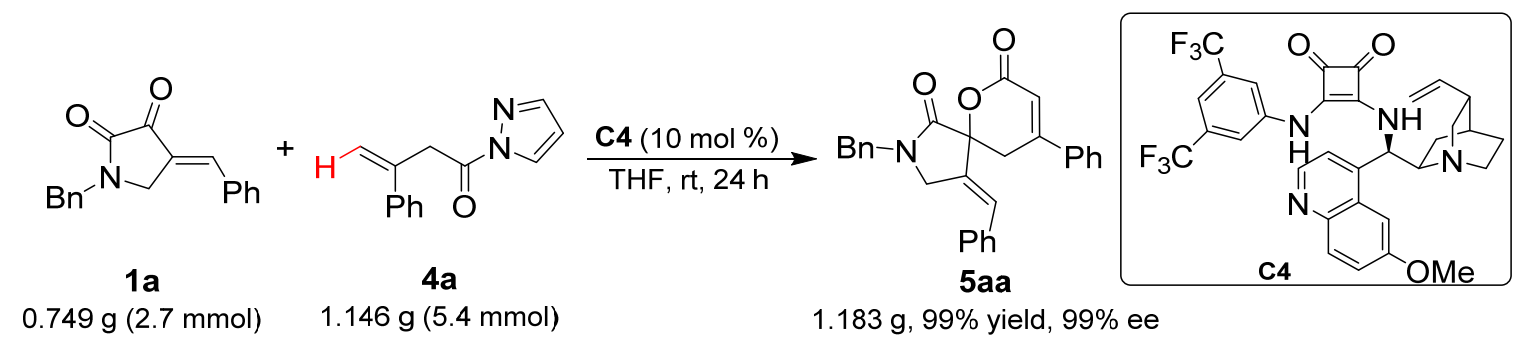


7. X-ray crystallographic analyses

7.1. Crystallographic information of $3 \mathrm{da}$

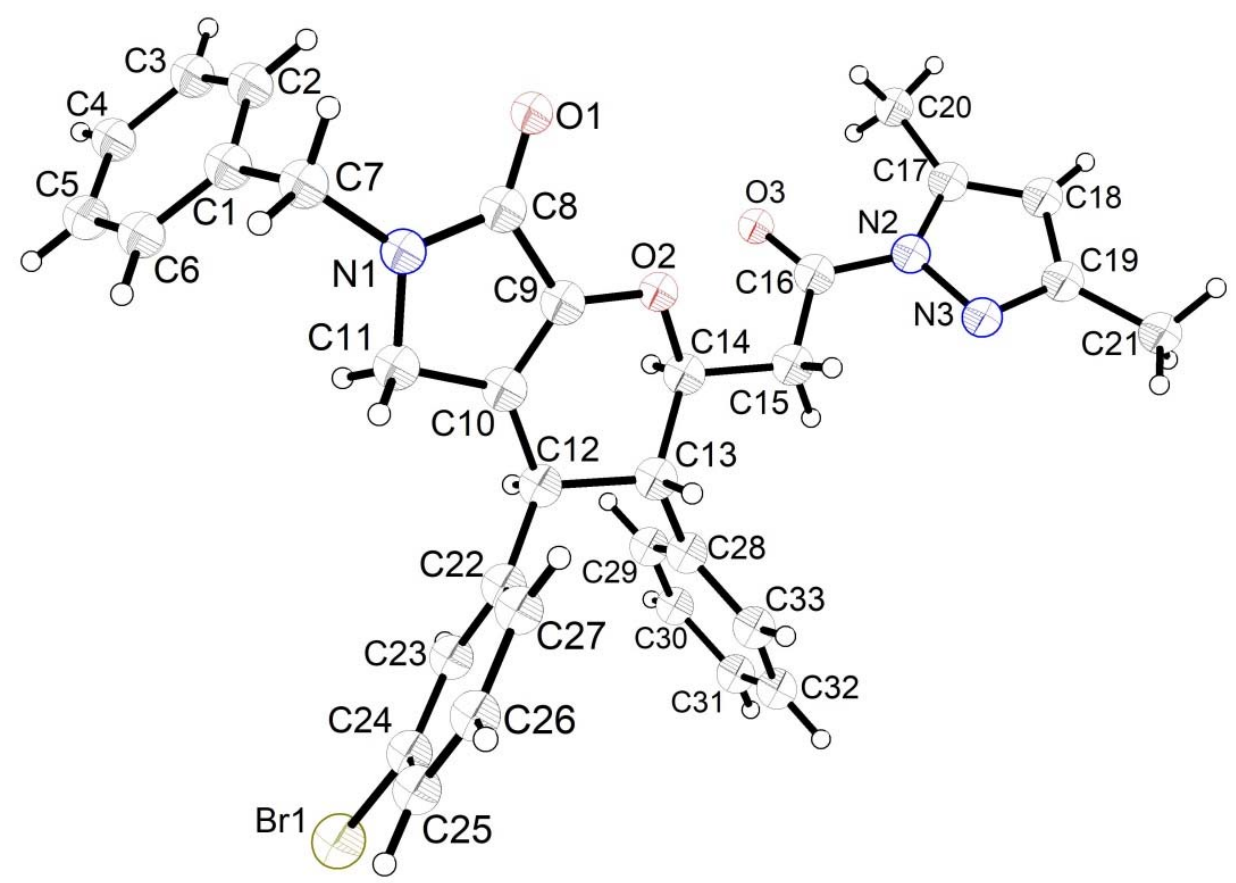

CCDC 1893188 (3ea) 
CCDC

Identification code

3da

Empirical formula

$\mathrm{C}_{33} \mathrm{H}_{30} \mathrm{BrN}_{3} \mathrm{O}_{3}$

Formula weight

596.53

Temperature/K

99.99(10)

Crystal system

triclinic

Space group

P-1

$\mathrm{a} / \AA ̊$

9.7162(2)

$\mathrm{b} / \AA$

$12.0758(2)$

$\mathrm{c} / \AA$

12.5323(3)

$\alpha /{ }^{\circ}$

103.486(2)

$\beta /{ }^{\circ}$

$99.856(2)$

$\gamma /{ }^{\circ}$

96.304(2)

Volume $/ \AA^{3}$

1391.47(5)

Z

2

$\rho_{\text {calcg }} / \mathrm{cm}^{3}$

1.4236

$\mu / \mathrm{mm}^{-1}$

2.331

$\mathrm{F}(000)$

616.3

Crystal size $/ \mathrm{mm}^{3}$

$0.2 \times 0.2 \times 0.1$

Radiation

$\mathrm{Cu} \mathrm{K \alpha}(\lambda=1.54184)$

$2 \Theta$ range for data collection $/{ }^{\circ} 7.4$ to 153.86

Index ranges

$-12 \leq \mathrm{h} \leq 12,-15 \leq \mathrm{k} \leq 15,-15 \leq 1 \leq 14$

Reflections collected

17158

Independent reflections

$5616\left[\mathrm{R}_{\text {int }}=0.0288, \mathrm{R}_{\text {sigma }}=0.0278\right]$

Data/restraints/parameters

$5616 / 0 / 363$

Goodness-of-fit on $\mathrm{F}^{2}$ 1.038

Final $R$ indexes $[\mathrm{I}>=2 \sigma(\mathrm{I})] \quad \mathrm{R}_{1}=0.0310, \mathrm{wR}_{2}=0.0783$

Final $\mathrm{R}$ indexes [all data] $\quad \mathrm{R}_{1}=0.0321, \mathrm{wR}_{2}=0.0790$

Largest diff. peak/hole / e $\AA^{-3} 0.38 /-0.62$ 
7.2. Crystallographic information of 5la

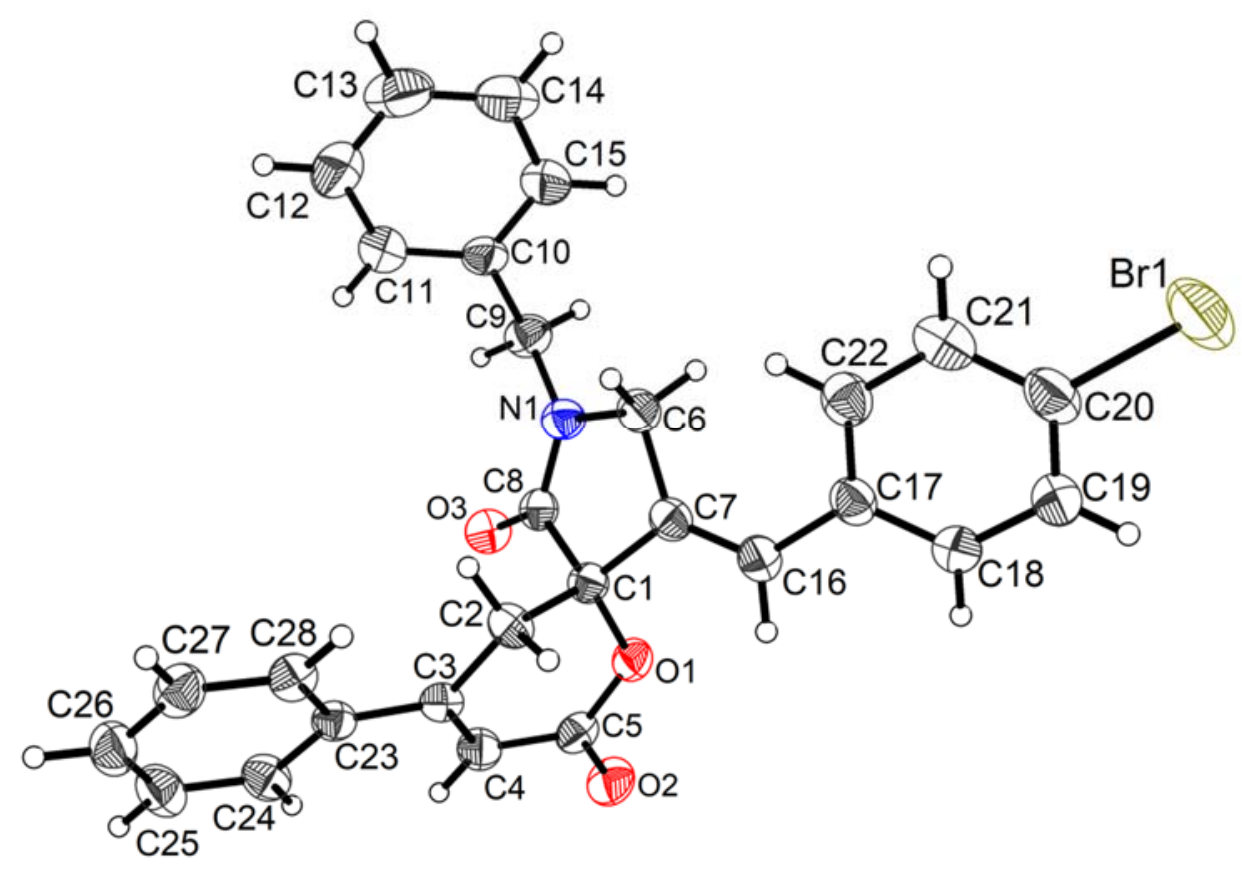

CCDC1913009 (5la) 
Identification code

Empirical formula

Formula weight

Temperature/K

Crystal system

Space group

$\mathrm{a} / \AA$

$\mathrm{b} / \AA$

$\mathrm{c} / \AA ̊$

$\alpha /{ }^{\circ}$

$\beta /{ }^{\circ}$

$\gamma /{ }^{\circ}$

Volume $/ \AA^{3}$

Z

$\rho_{\text {calc }} / \mathrm{cm}^{3}$

$\mu / \mathrm{mm}^{-1}$

$\mathrm{F}(000)$

Crystal size $/ \mathrm{mm}^{3}$

Radiation 5la

$\mathrm{C}_{28} \mathrm{H}_{22} \mathrm{BrNO}_{3}$

500.37

213.99(11)

orthorhombic

$\mathrm{P} 2{ }_{12} 2_{1}$

5.53640(10)

16.4802(2)

25.7867(3)

90

90

90

2352.80(6)

4

1.413

2.621

1024.0

$0.15 \times 0.1 \times 0.1$

$\mathrm{CuK} \alpha(\lambda=1.54184)$

$2 \Theta$ range for data collection $/{ }^{\circ} 6.364$ to 153.178

Index ranges

$-6 \leq \mathrm{h} \leq 6,-19 \leq \mathrm{k} \leq 20,-25 \leq 1 \leq 31$

Reflections collected 12966

Independent reflections $4707\left[\mathrm{R}_{\text {int }}=0.0343, \mathrm{R}_{\text {sigma }}=0.0335\right]$

Data/restraints/parameters

$4707 / 0 / 298$

Goodness-of-fit on $\mathrm{F}^{2}$

1.045

Final $R$ indexes $[\mathrm{I}>=2 \sigma(\mathrm{I})] \quad \mathrm{R}_{1}=0.0341, \mathrm{wR}_{2}=0.0870$

Final $\mathrm{R}$ indexes [all data] $\quad \mathrm{R}_{1}=0.0353, \mathrm{wR}_{2}=0.0881$

Largest diff. peak/hole / e $\AA^{-3} 0.56 /-0.74$

Flack parameter $-0.044(9)$ 


\section{Transformation of vinylogous-cyclization products}<smiles>CC[13C](=O)c1ccccc1</smiles>

\subsection{Synthesis of ester $6^{[1]}$}

Compound 3aa (104.0 mg, $0.2 \mathrm{mmol})$ and DBU (7.0 mg, $0.048 \mathrm{mmol})$ in $2 \mathrm{~mL}$ $\mathrm{MeOH}$ were stirred for 6 hours at room temperature. After removal of the solvent under reduced pressure, the reaction mixture was purified by silica gel column chromatography (hexane/EtOAc $=4 / 1$ ) to give product 6 .

methyl 2-((2S,3S,4S)-6-benzyl-7-oxo-3,4-diphenyl-2,3,4,5,6,7-hexahydropyrano [2,3-c]pyrrol-2-yl)acetate (6)<smiles>COC(=O)C[C@@H]1OC2=C(CN(Cc3ccccc3)C2=O)[C@H](c2ccccc2)[C@H]1c1ccccc1</smiles>

White solid, $77.9 \mathrm{mg}, 86 \%$ yield, $\mathrm{mp}=119.9-120.7{ }^{\circ} \mathrm{C},[\alpha] \mathrm{D}^{25}=-64.5$ (c 1.484, $\left.\mathrm{CH}_{2} \mathrm{Cl}_{2}\right), 95 \%$ ee.

${ }^{1} \mathrm{H}$ NMR (400 MHz, $\left.\mathrm{CDCl}_{3}\right) \delta 7.32-7.23(\mathrm{~m}, 3 \mathrm{H}), 7.22-7.15(\mathrm{~m}, 5 \mathrm{H}), 7.14-7.09$ $(\mathrm{m}, 3 \mathrm{H}), 6.93(\mathrm{~d}, J=6.5 \mathrm{~Hz}, 2 \mathrm{H}), 6.85-6.78(\mathrm{~m}, 2 \mathrm{H}), 4.90-4.77(\mathrm{~m}, 2 \mathrm{H}), 4.34(\mathrm{~d}, J$ $=15.0 \mathrm{~Hz}, 1 \mathrm{H}), 3.82(\mathrm{~d}, J=10.1 \mathrm{~Hz}, 1 \mathrm{H}), 3.52(\mathrm{~d}, J=23.5 \mathrm{~Hz}, 4 \mathrm{H}), 3.39(\mathrm{~d}, J=18.2$ $\mathrm{Hz}, 1 \mathrm{H}), 3.04(\mathrm{t}, J=10.4 \mathrm{~Hz}, 1 \mathrm{H}), 2.61(\mathrm{dd}, J=16.3,7.3 \mathrm{~Hz}, 1 \mathrm{H}), 2.46(\mathrm{dd}, J=16.3$, $3.8 \mathrm{~Hz}, 1 \mathrm{H})$.

${ }^{13} \mathrm{C}$ NMR $\left(100 \mathrm{MHz}, \mathrm{CDCl}_{3}\right) \delta 170.6,164.9,146.0,139.5,138.5,137.1,128.8,128.7$, $128.6,128.4$, 128.0, 128.0, 127.6, 127.5, 127.2, 124.2, 78.3, 52.9, 51.8, 47.3, 47.2, $46.5,38.1$.

HRMS (ESI) $m / z$ calculated for $\mathrm{C}_{29} \mathrm{H}_{28} \mathrm{NO}_{4}{ }^{+}[\mathrm{M}+\mathrm{H}]^{+}:$454.2013, found 454.2006. 
HPLC analysis: (IA column, Hexane:2-propanol $=80: 20$, flow rate $=0.4 \mathrm{~mL} / \mathrm{min}$, wavelength $=254 \mathrm{~nm}$ ): $R \mathrm{t}=34.66$ (major), 26.83 (minor).

\subsection{Synthesis of amide $7^{[2]}$}

Compound 3aa $(51.7 \mathrm{mg}, 0.1 \mathrm{mmol})$ and benzylamine $(21.4 \mathrm{mg}, 0.2 \mathrm{mmol})$ in 1 $\mathrm{mL}$ THF were stirred for 6 hours at $50^{\circ} \mathrm{C}$. After removal of the solvent under reduced pressure, the reaction mixture was purified by silica gel column chromatography (hexane/EtOAc $=1 / 1)$ to give product 7.

$N$-benzyl-2-((2S,3S,4S)-6-benzyl-7-oxo-3,4-diphenyl-2,3,4,5,6,7-hexahydropyrano [2,3-c]pyrrol-2-yl)acetamide (7)<smiles>O=C(CC1OC2=C(CN(Cc3ccccc3)C2=O)[C@H](c2ccccc2)[C@@H]1c1ccccc1)NCc1ccccc1</smiles>

White solid, $43.6 \mathrm{mg}, 82 \%$ yield, $\mathrm{mp}=127.2-128.0{ }^{\circ} \mathrm{C},[\alpha] \mathrm{D}^{25}=-22.1$ (c 0.383 , $\left.\mathrm{CH}_{2} \mathrm{Cl}_{2}\right), 98 \%$ ee.

${ }^{1} \mathrm{H}$ NMR (400 MHz, $\left.\mathrm{CDCl}_{3}\right) \delta 7.34-7.29(\mathrm{~m}, 5 \mathrm{H}), 7.27(\mathrm{~d}, J=11.5 \mathrm{~Hz}, 2 \mathrm{H}), 7.18(\mathrm{~d}$, $J=7.4 \mathrm{~Hz}, 5 \mathrm{H}), 7.11(\mathrm{~d}, J=4.4 \mathrm{~Hz}, 3 \mathrm{H}), 6.91(\mathrm{~d}, J=6.3 \mathrm{~Hz}, 2 \mathrm{H}), 6.80-6.72(\mathrm{~m}$, $2 \mathrm{H}), 6.65-6.57(\mathrm{~m}, 1 \mathrm{H}), 4.81(\mathrm{dt}, J=9.1,5.4 \mathrm{~Hz}, 2 \mathrm{H}), 4.48-4.40(\mathrm{~m}, 1 \mathrm{H}), 4.36(\mathrm{dd}$, $J=14.5,9.0 \mathrm{~Hz}, 1 \mathrm{H}), 3.77(\mathrm{~d}, J=10.0 \mathrm{~Hz}, 1 \mathrm{H}), 3.51(\mathrm{~d}, J=18.2 \mathrm{~Hz}, 1 \mathrm{H}), 3.38$ (d, $J$ $=18.2 \mathrm{~Hz}, 1 \mathrm{H}), 2.96(\mathrm{t}, J=10.3 \mathrm{~Hz}, 1 \mathrm{H}), 2.41(\mathrm{~d}, J=4.6 \mathrm{~Hz}, 2 \mathrm{H}), 2.02(\mathrm{~d}, J=4.1 \mathrm{~Hz}$, $1 \mathrm{H})$.

${ }^{13} \mathrm{C}$ NMR $\left(100 \mathrm{MHz}, \mathrm{CDCl}_{3}\right) \delta 169.4,165.0,145.7,139.4,138.6,138.4,137.0,128.8$, $128.8,128.6,128.5,128.0,127.8,127.6,127.4,127.2,124.5,79.0,52.9,47.5,47.4$, $46.5,43.6,40.2$.

HRMS (ESI) $m / z$ calculated for $\mathrm{C}_{35} \mathrm{H}_{33} \mathrm{~N}_{2} \mathrm{O}_{3}{ }^{+}[\mathrm{M}+\mathrm{H}]^{+}:$529.2486, found 529.2479.

HPLC analysis: (IA column, Hexane:EtOH $=45: 55$, flow rate $=0.3 \mathrm{~mL} / \mathrm{min}$, wavelength $=254 \mathrm{~nm}$ ): $R \mathrm{t}=37.58$ (major), 30.15 (minor).

\subsection{Synthesis of alcohol $8^{[2]}$}

Compound 3aa (103.5 mg, $0.2 \mathrm{mmol}, 1.0$ equiv.) in THF (3.08 mL) and $\mathrm{H}_{2} \mathrm{O}$ $(0.78 \mathrm{~mL})$ were cooled to $0{ }^{\circ} \mathrm{C}$, then $\mathrm{NaBH}_{4}(60.5 \mathrm{mg}, 1.6 \mathrm{mmol}, 8.0$ equiv.) was added. The reaction mixture was allowed to warm up to room temperature and stirred for additional 2 hours. After that, the reaction was quenched by $1 \mathrm{~N} \mathrm{HCl}(10.0 \mathrm{~mL})$ and extracted with $\mathrm{CH}_{2} \mathrm{Cl}_{2}(15 \mathrm{~mL} \times 3)$. The combined organic layers were dried over $\mathrm{Na}_{2} \mathrm{SO}_{4}$. After removal of the solvent under reduced pressure, the crude was purified by silica gel column chromatography (hexane/EtOAc $=1 / 1)$ to give product 8 . 
(2S,3S,4S)-6-benzyl-2-(2-hydroxyethyl)-3,4-diphenyl-3,4,5,6-tetrahydropyrano[2, 3-c]pyrrol-7(2H)-one (8)<smiles>O=C1C2=C(CN(Cc3ccccc3)C2=O)[C@H](c2ccccc2)[C@@H](c2ccccc2)[C@@H]1CCO</smiles>

White solid, $65.0 \mathrm{mg}, 76 \%$ yield, $\mathrm{mp}=83.5-83.7{ }^{\circ} \mathrm{C},[\alpha] \mathrm{D}^{25}=-41.7$ (c 0.620 , $\left.\mathrm{CH}_{2} \mathrm{Cl}_{2}\right),>99 \%$ ee.

${ }^{1} \mathrm{H}$ NMR $\left(400 \mathrm{MHz}, \mathrm{CDCl}_{3}\right) \delta 7.32-7.25(\mathrm{~m}, 3 \mathrm{H}), 7.22-7.15(\mathrm{~m}, 5 \mathrm{H}), 7.14-7.10$ (m, 3H), $6.92-6.87(\mathrm{~m}, 2 \mathrm{H}), 6.79(\mathrm{dd}, J=6.4,2.9 \mathrm{~Hz}, 2 \mathrm{H}), 4.84(\mathrm{~d}, J=15.0 \mathrm{~Hz}, 1 \mathrm{H})$, $4.52(\mathrm{td}, J=10.3,3.3 \mathrm{~Hz}, 1 \mathrm{H}), 4.35(\mathrm{~d}, J=15.0 \mathrm{~Hz}, 1 \mathrm{H}), 3.93-3.83(\mathrm{~m}, 1 \mathrm{H}), 3.76$ $(\mathrm{dd}, J=10.7,6.0 \mathrm{~Hz}, 2 \mathrm{H}), 3.52(\mathrm{dd}, J=18.2,1.3 \mathrm{~Hz}, 1 \mathrm{H}), 3.42(\mathrm{~d}, J=18.2 \mathrm{~Hz}, 1 \mathrm{H})$, $2.88(\mathrm{t}, J=10.3 \mathrm{~Hz}, 1 \mathrm{H}), 2.50(\mathrm{~s}, 1 \mathrm{H}), 2.14(\mathrm{~s}, 1 \mathrm{H}), 1.75-1.61(\mathrm{~m}, 2 \mathrm{H})$.

${ }^{13} \mathrm{C} \mathrm{NMR}\left(100 \mathrm{MHz}, \mathrm{CDCl}_{3}\right) \delta 165.3,145.9,139.7,139.3,137.0,128.7,128.5,128.2$, $128.1,128.0,128.0,127.6,127.2,127.1,124.3,79.8,59.0,53.9,47.6,47.4,46.5$, 35.5 .

HRMS (ESI) $m / z$ calculated for $\mathrm{C}_{28} \mathrm{H}_{28} \mathrm{NO}_{3}{ }^{+}[\mathrm{M}+\mathrm{H}]^{+}: 426.2064$, found 426.2058.

HPLC analysis: (IC column, Hexane:2-propanol $=50: 50$, flow rate $=0.3 \mathrm{~mL} / \mathrm{min}$, wavelength $=254 \mathrm{~nm}$ ): $R \mathrm{t}=72.76$ (major), 66.41 (minor).

\subsection{Synthesis of $9^{[3]}$}

Compound 5aa ( $84.0 \mathrm{mg}, 0.2 \mathrm{mmol}, 1.0$ equiv.) in THF ( $2 \mathrm{~mL})$ were cooled to 0 ${ }^{\circ} \mathrm{C}$, then $\mathrm{LiAlH}_{4}(38.0 \mathrm{mg}, 1.0 \mathrm{mmol}, 5.0$ equiv.) was added. The reaction mixture was stirred at $0{ }^{\circ} \mathrm{C}$ for 6 hours. After that, the reaction was quenched by $\mathrm{H}_{2} \mathrm{O}(2 \mathrm{~mL})$ and extracted with $\mathrm{CH}_{2} \mathrm{Cl}_{2}(1 \mathrm{~mL} \times 3)$. The combined organic layers were dried over $\mathrm{Na}_{2} \mathrm{SO}_{4}$. After removal of the solvent under reduced pressure, the crude was purified by silica gel column chromatography (hexane/EtOAc $=8 / 1$ ) to give product 9 .

(S,E)-2-benzyl-4-benzylidene-9-phenyl-6-oxa-2-azaspiro[4.5]dec-8-ene (9)<smiles>C1=C(c2ccccc2)C[C@]2(CN(Cc3ccccc3)C/C2=C/c2ccccc2)OC1</smiles>

White solid, $46.6 \mathrm{mg}, 59 \%$ yield, $\mathrm{mp}=146.5-147.4{ }^{\circ} \mathrm{C},[\alpha] \mathrm{D}^{25}=-5.5(\mathrm{c} 0.427$, $\mathrm{CH}_{2} \mathrm{Cl}_{2}$ ), $98 \%$ ee.

${ }^{1} \mathrm{H}$ NMR $\left(400 \mathrm{MHz}, \mathrm{CDCl}_{3}\right) \delta 7.48(\mathrm{~d}, J=7.7 \mathrm{~Hz}, 2 \mathrm{H}), 7.39-7.30(\mathrm{~m}, 9 \mathrm{H}), 7.21(\mathrm{dd}$, $J=12.8,7.3 \mathrm{~Hz}, 4 \mathrm{H}), 6.64$ (s, 1H), 5.63 (s, 1H), 4.67 (d, J=17.5 Hz, 1H), 4.36 (s, 
$1 \mathrm{H}), 4.18(\mathrm{~d}, J=17.4 \mathrm{~Hz}, 1 \mathrm{H}), 4.03-3.93(\mathrm{~m}, 4 \mathrm{H}), 3.41(\mathrm{~d}, J=16.6 \mathrm{~Hz}, 1 \mathrm{H}), 2.88(\mathrm{~s}$, 1H), 2.37 (s, 1H).

${ }^{13} \mathrm{C}$ NMR $\left(100 \mathrm{MHz}, \mathrm{CDCl}_{3}\right) \delta 145.2,144.3,138.1,138.1,136.9,128.9,128.5,128.4$, $128.4,128.3,127.2,127.2,126.9,126.3,125.7,123.9,98.7,86.2,68.9,54.7,52.9$, 39.0 .

HRMS (ESI) $m / z$ calculated for $\mathrm{C}_{28} \mathrm{H}_{27} \mathrm{KNO}^{+}[\mathrm{M}+\mathrm{K}]^{+}:$432.1724, found 432.1931 .

HPLC analysis: (IA column, Hexane:2-propanol $=90: 10$, flow rate $=0.7 \mathrm{~mL} / \mathrm{min}$, wavelength $=254 \mathrm{~nm}$ ): $R \mathrm{t}=15.36$ (major), 17.19 (minor). 


\section{References}

[1] Luo, Y.; Xie, K.; Yue, D.; Zhang, X.; Xu, X.; Yuan, W. Org. Biomol. Chem. 2018, $16,3372$.

[2] Zhang, H.; Shi, C.; Zhong, F.; Yin, L. J. Am. Chem. Soc. 2017, 139, 2196.

[3] Yang, K.; Li, Q.; Liu, Y.; He, Q.; Liu, Y.; Leng, H.; Jia, A.; Ramachandram, S.; Long, J. Org. Lett. 2018, 20, 7518. 


\section{NMR spectra and HPLC chromatograms}

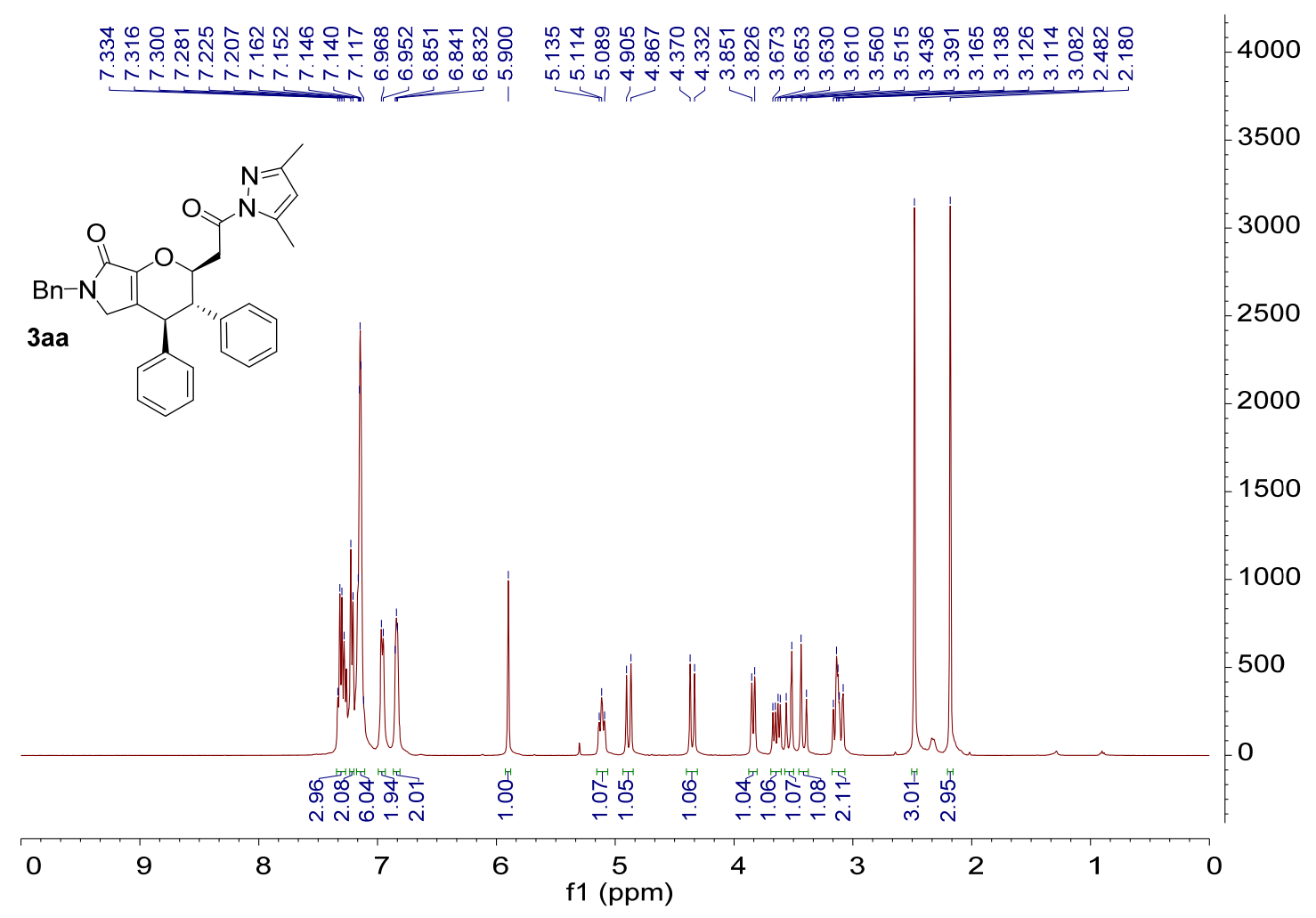

ㅎำ

임

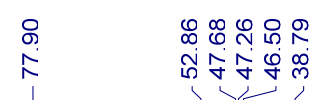

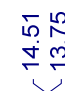

1500<smiles>Cc1cc(C)n(C(=O)CC2OC3=C(CN(Cc4ccccc4)C3=O)[C@@H](c3ccccc3)[C@H]2c2ccccc2)n1</smiles>
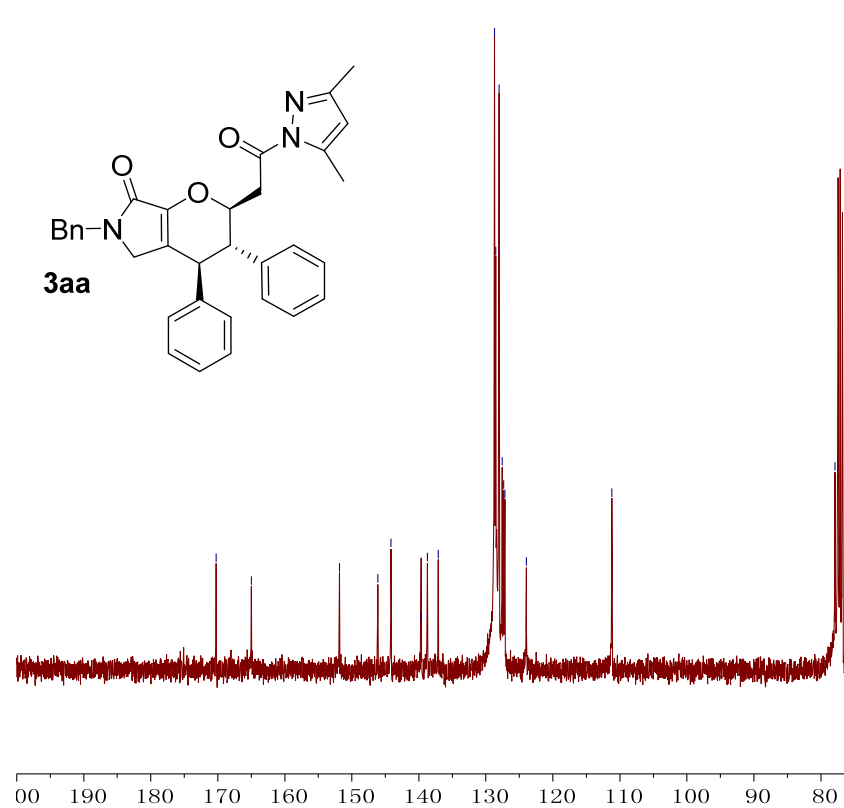

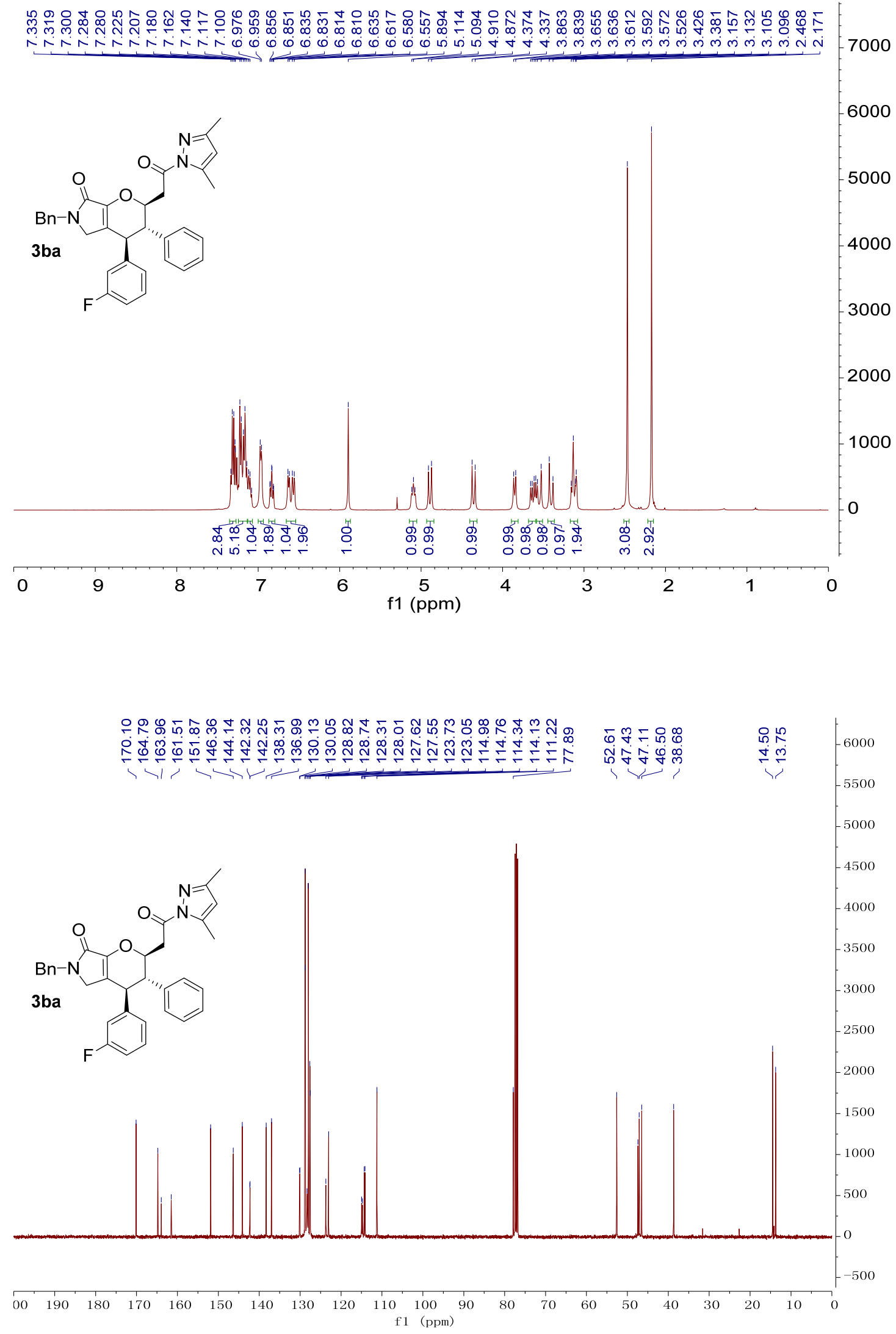

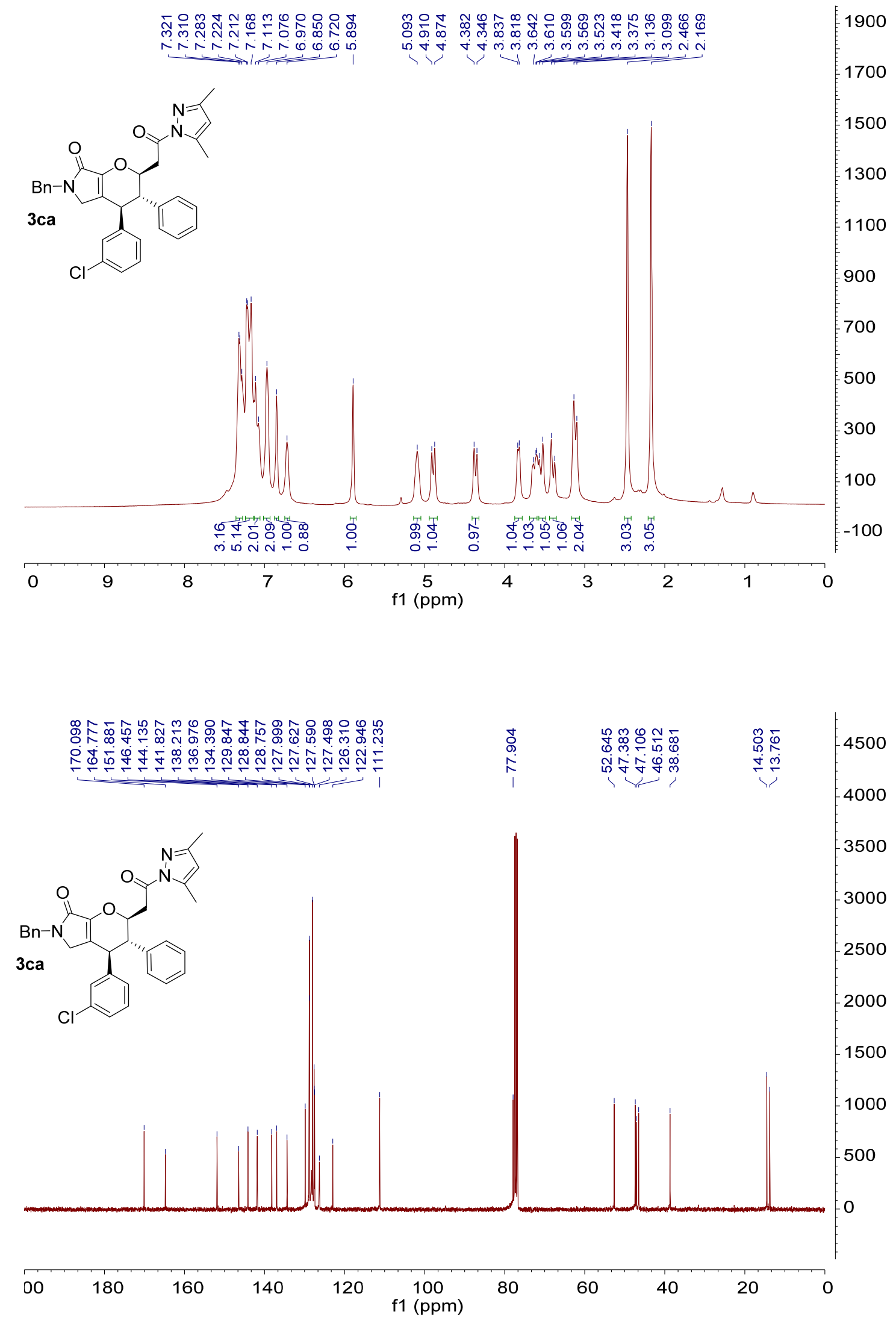


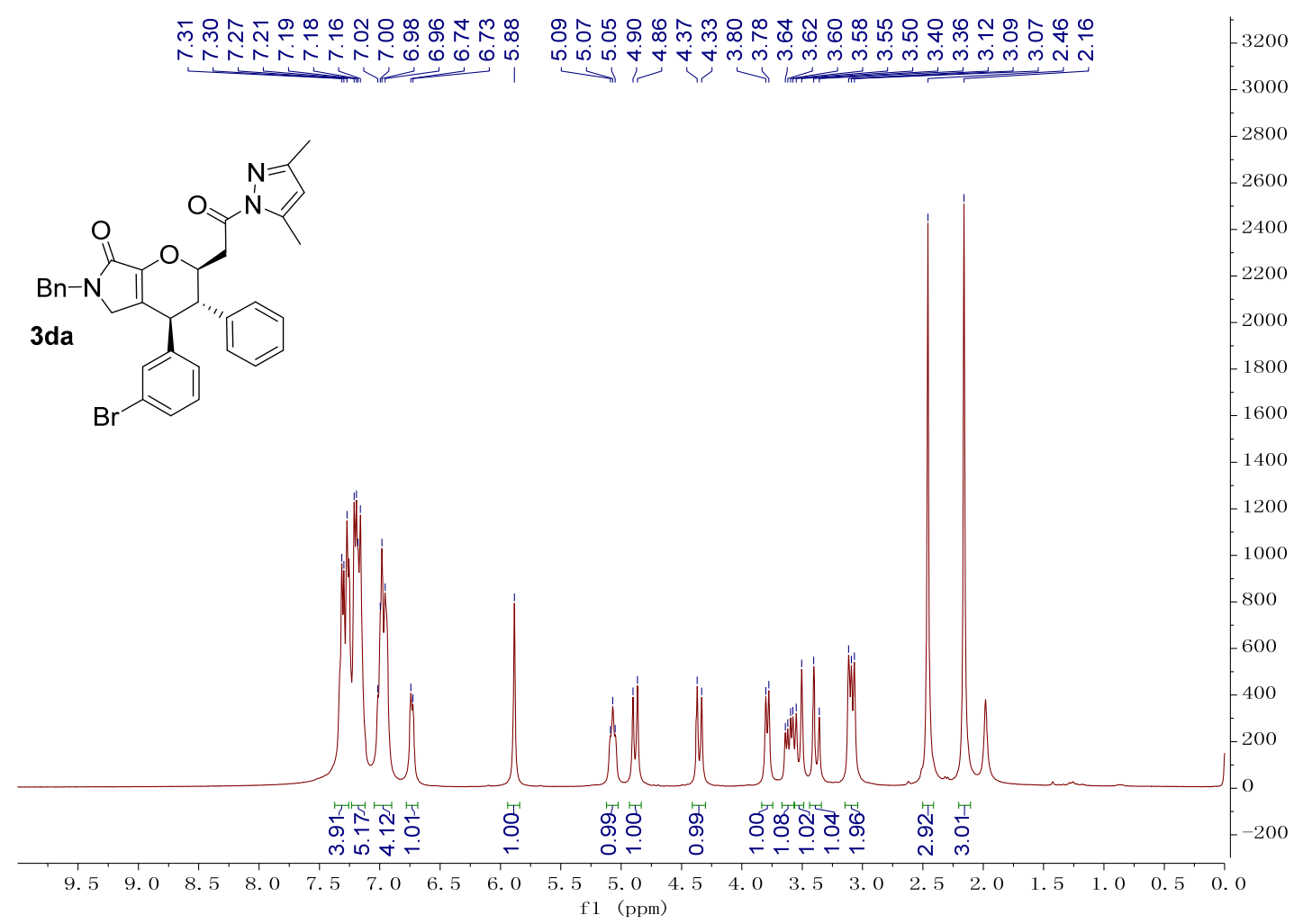

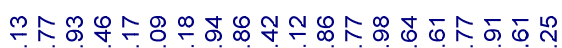

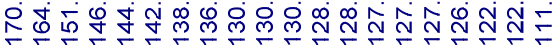

5. 04

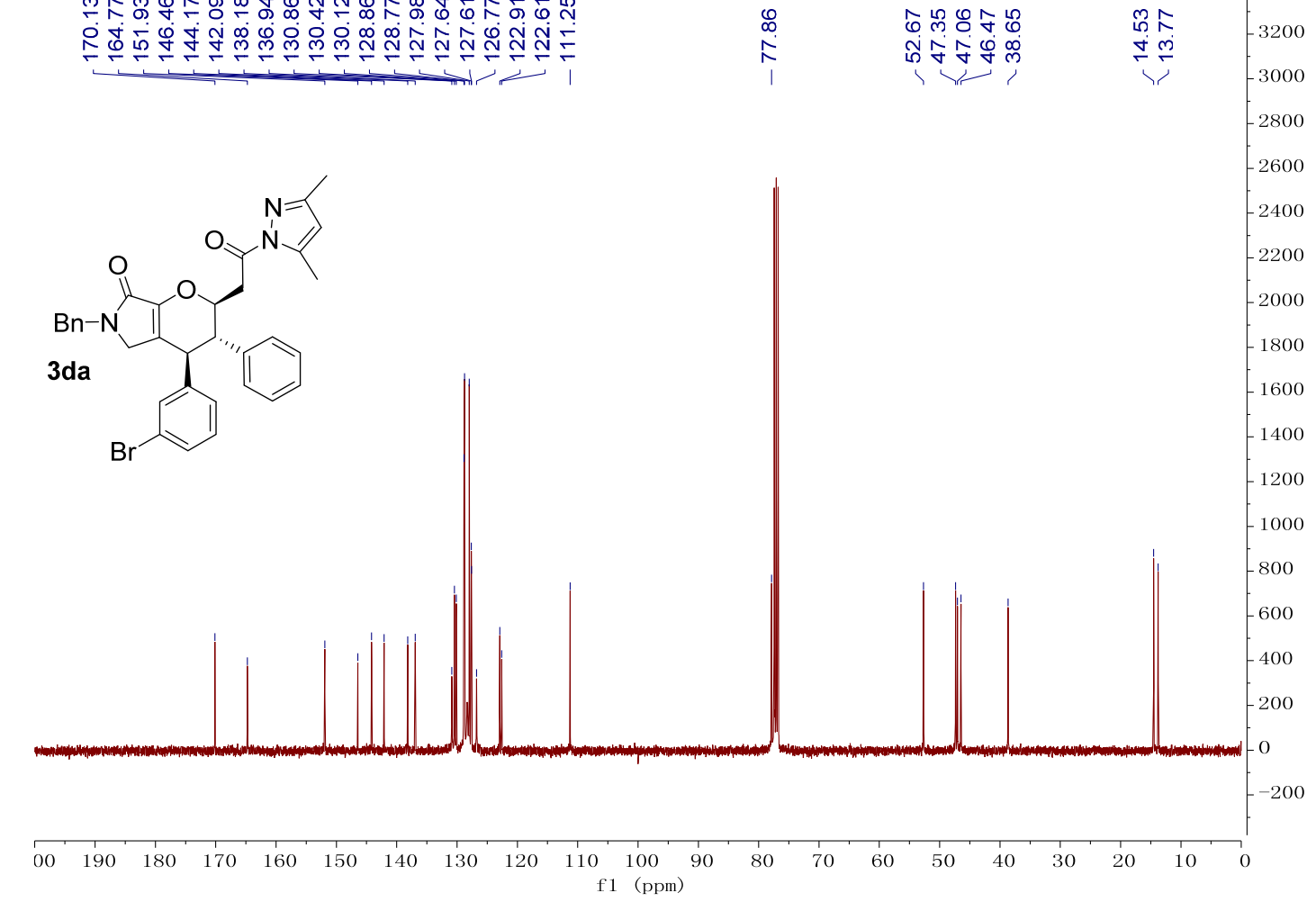



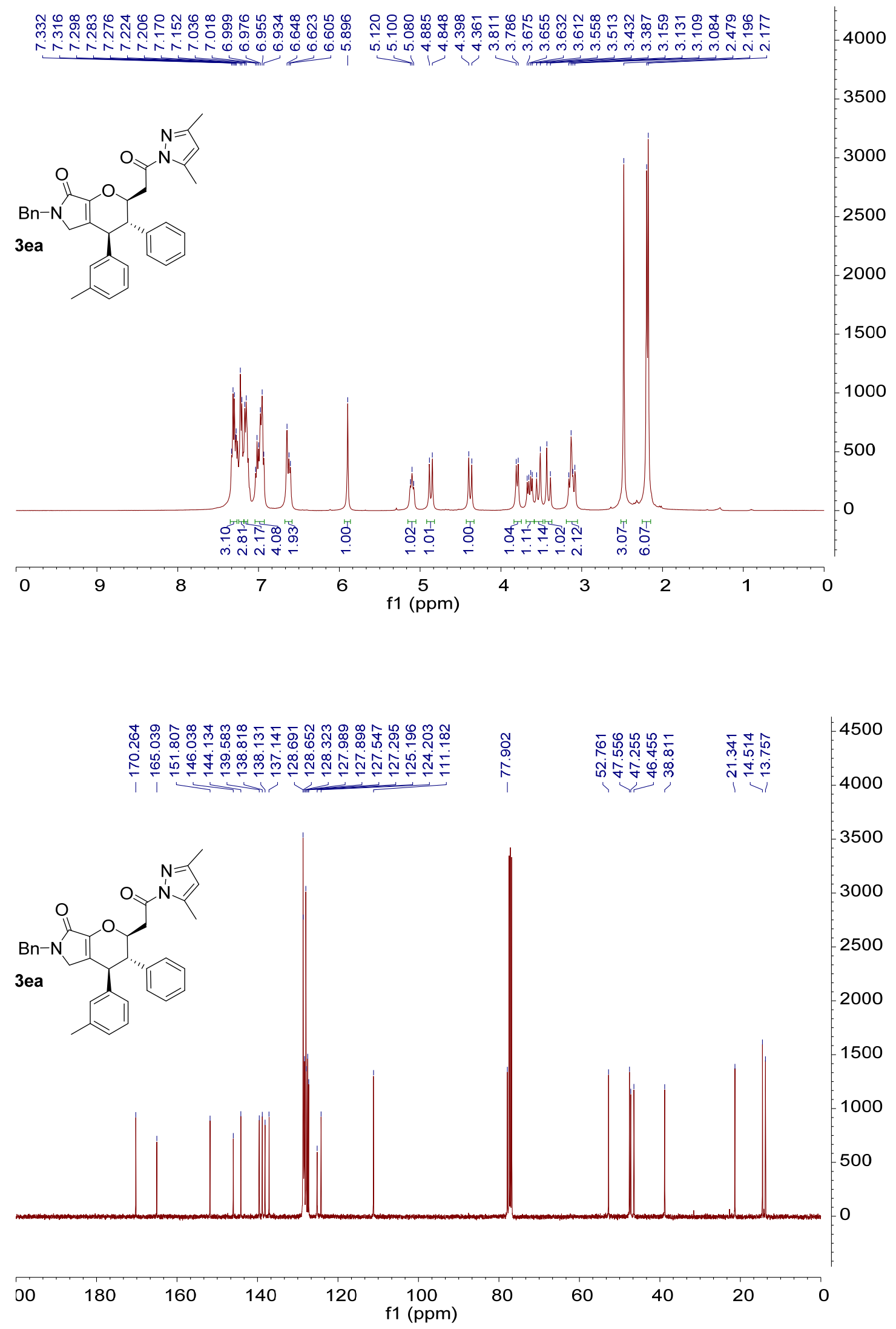


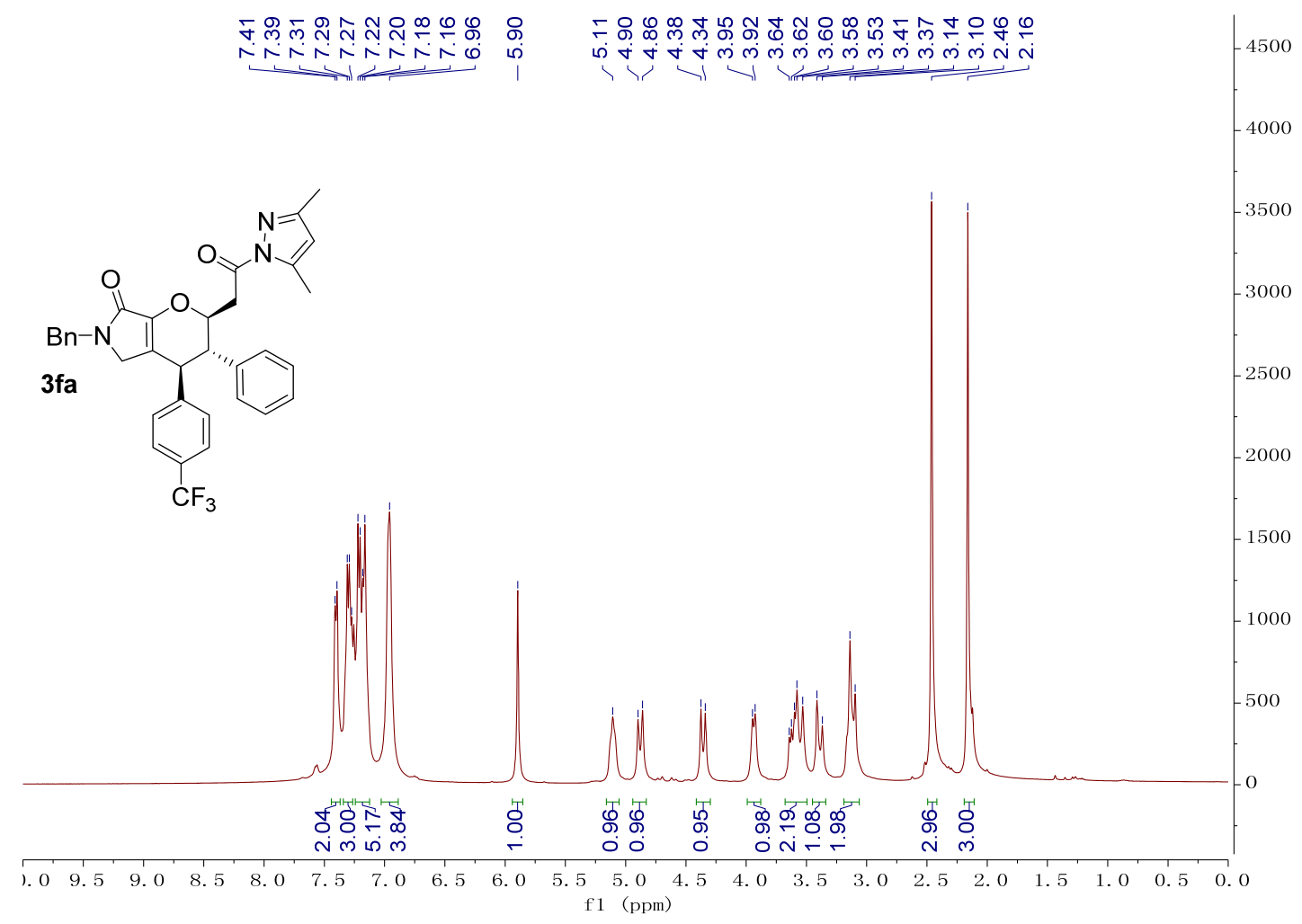

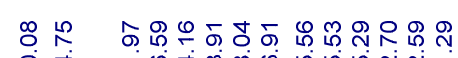

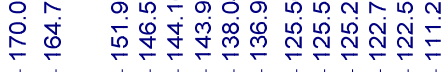

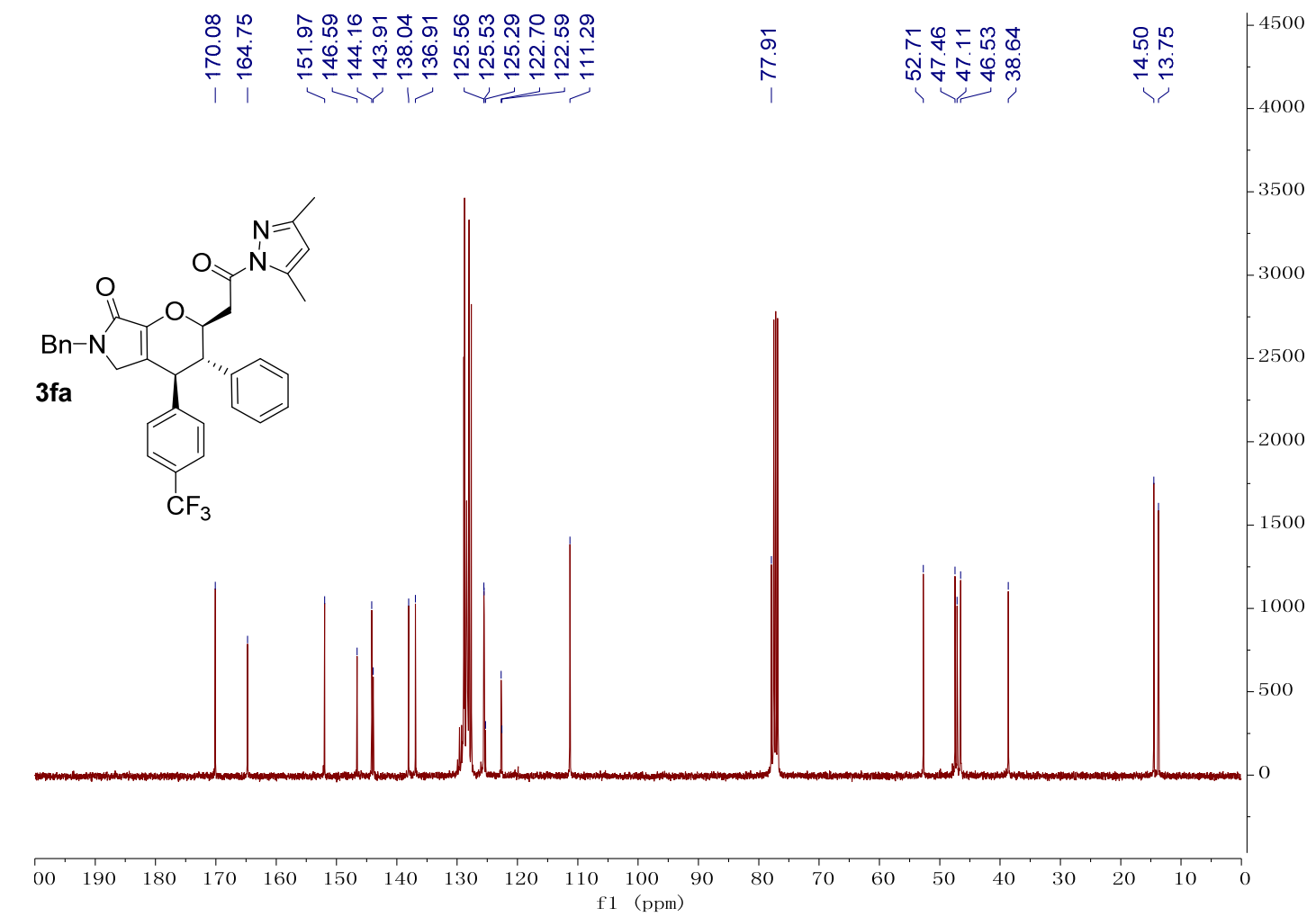



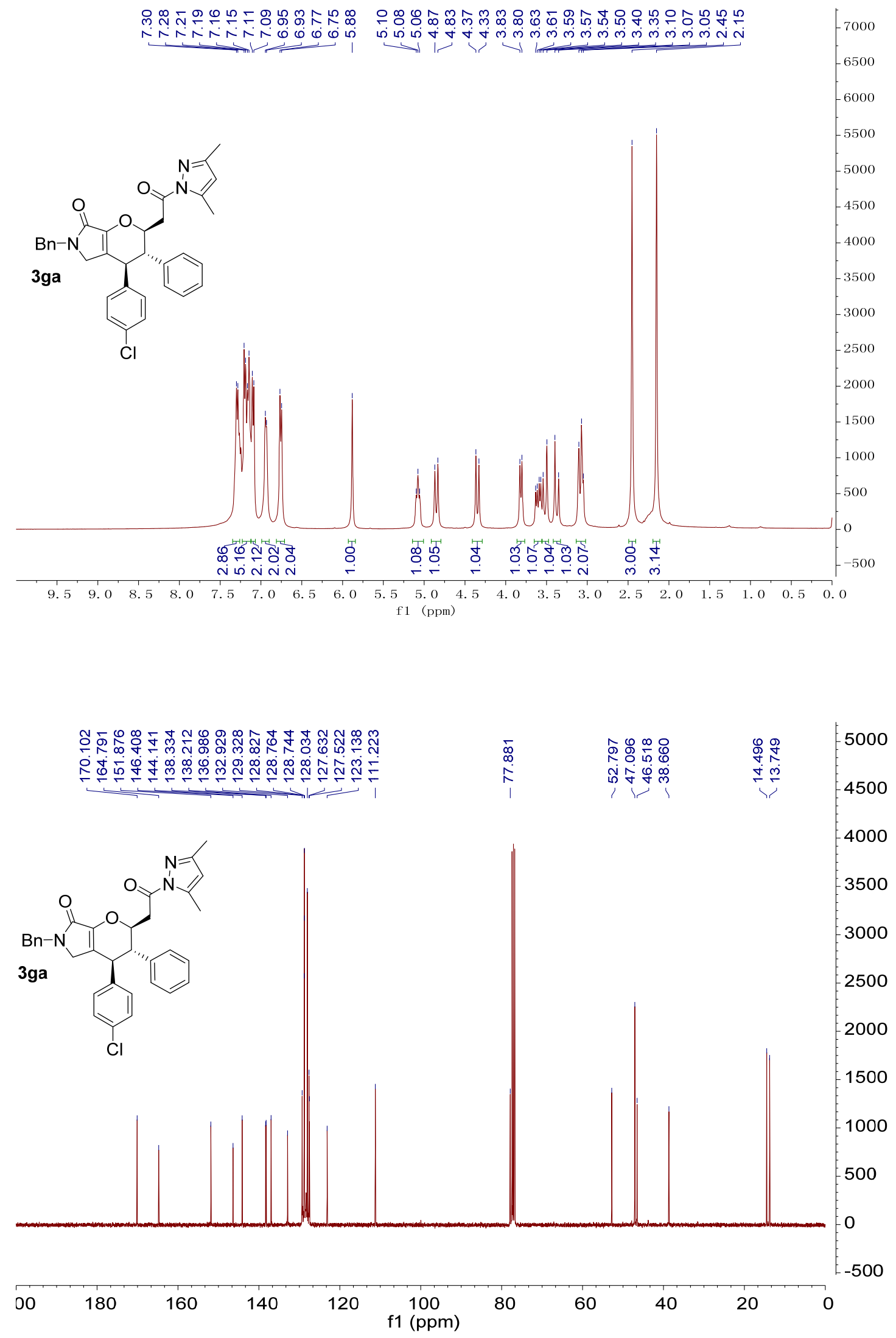

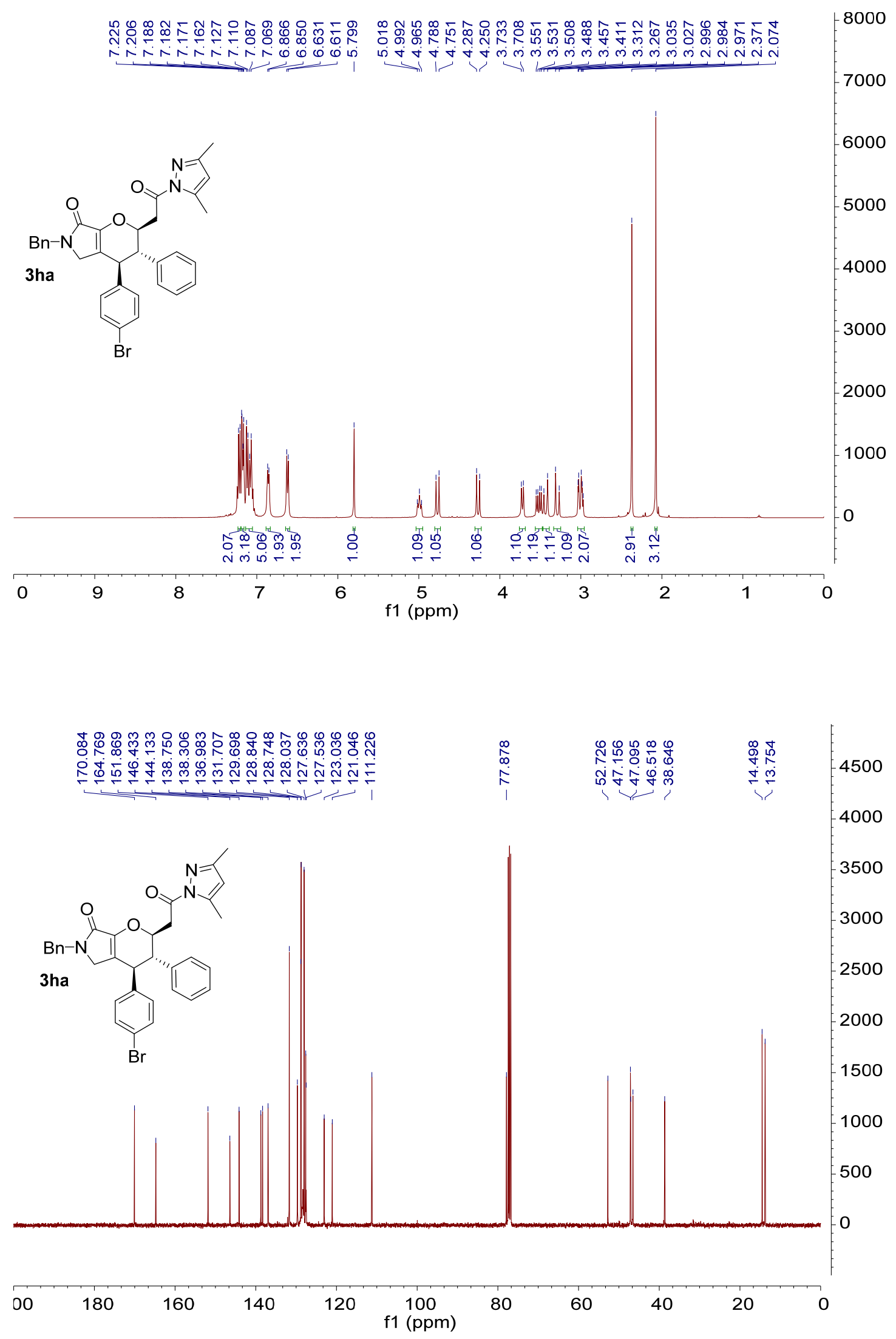

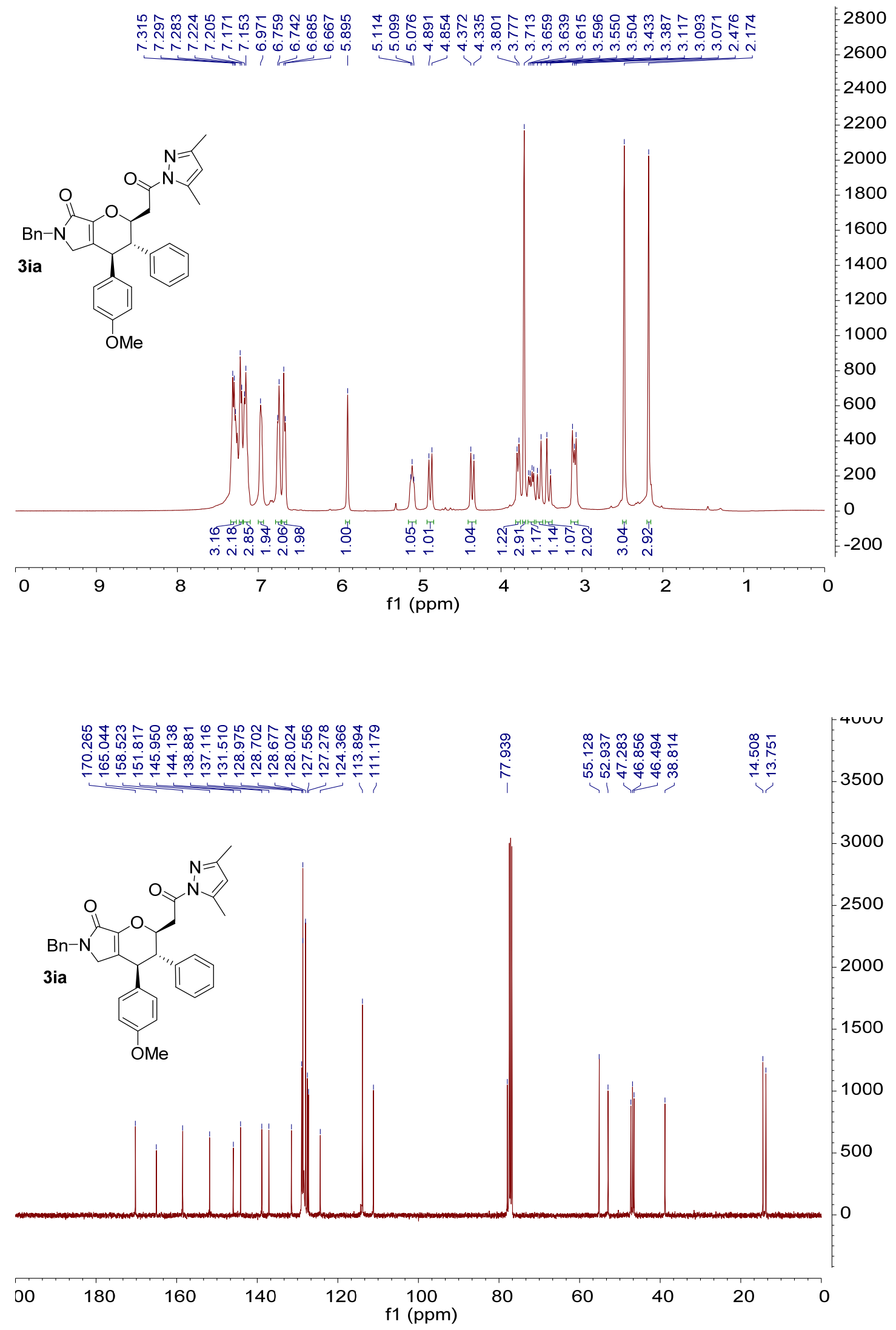

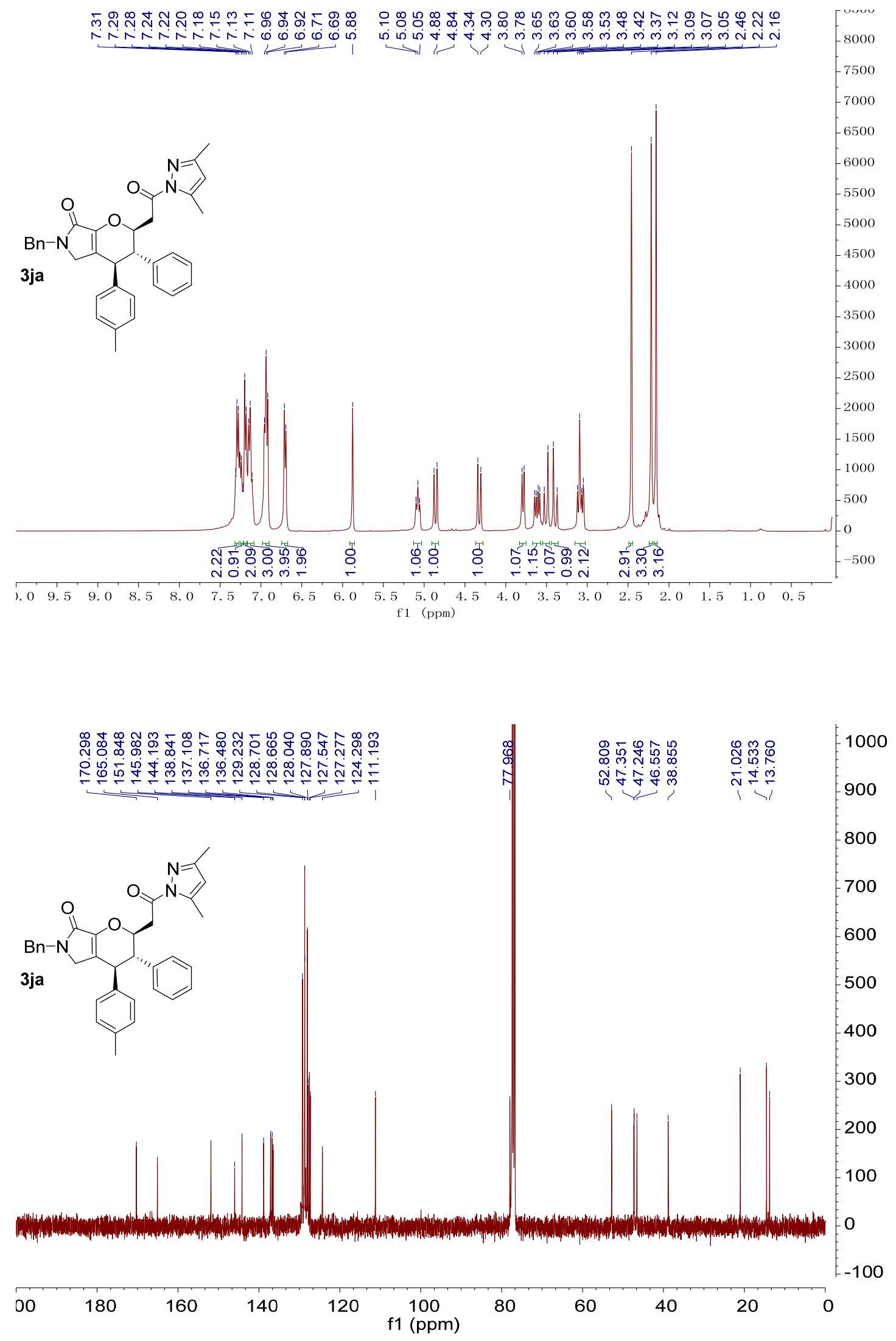

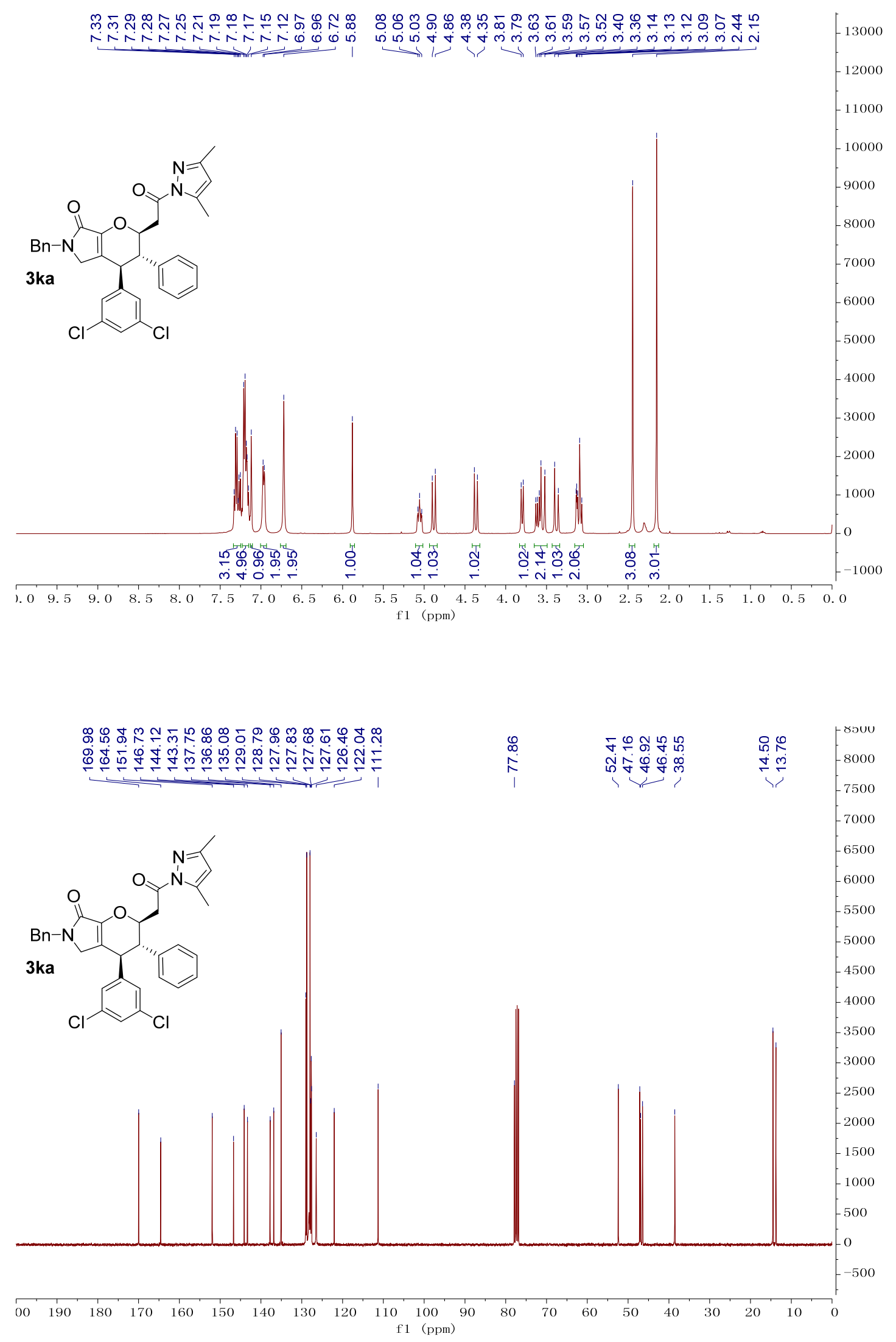


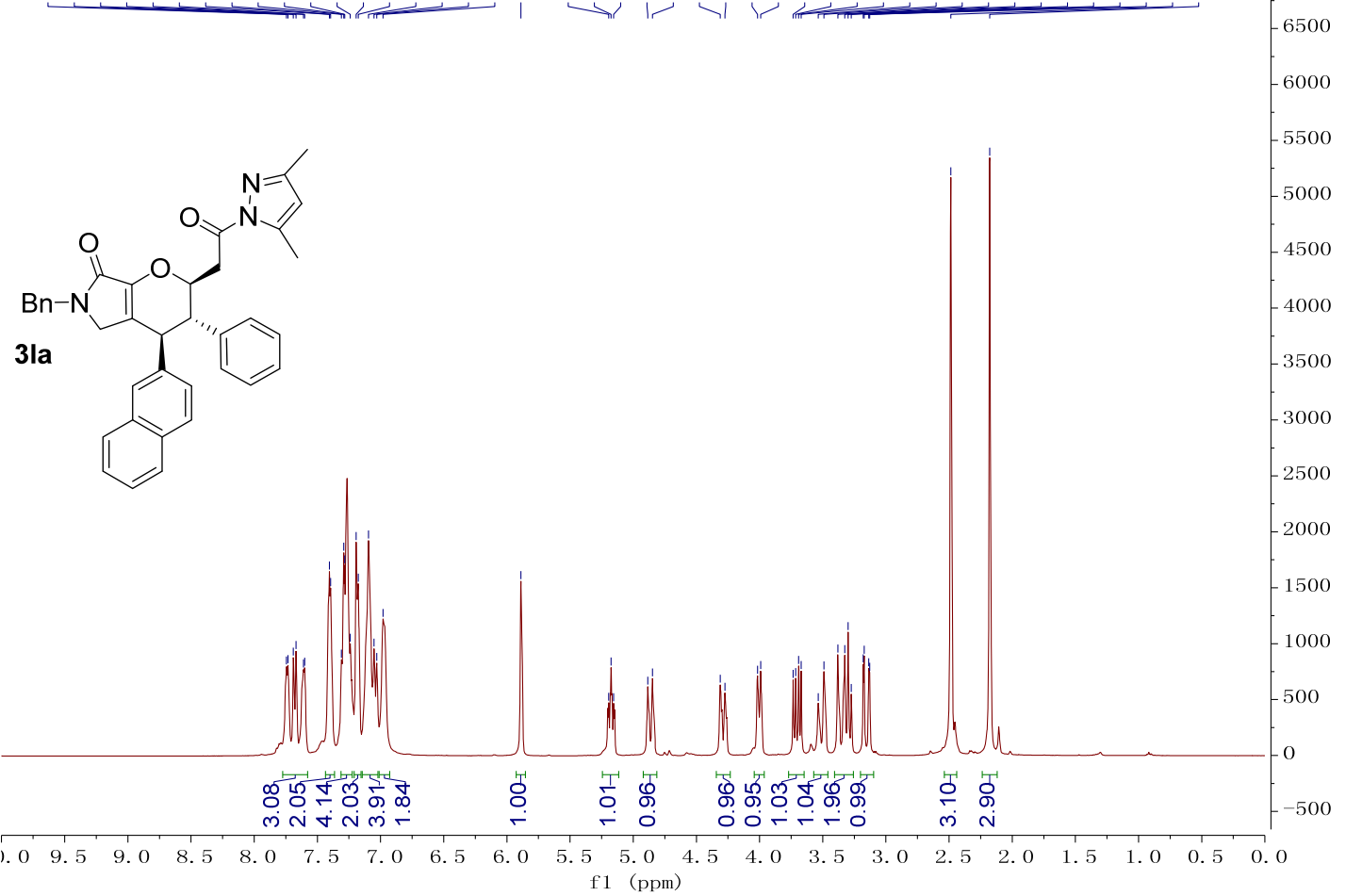

ำ

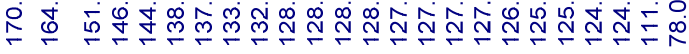

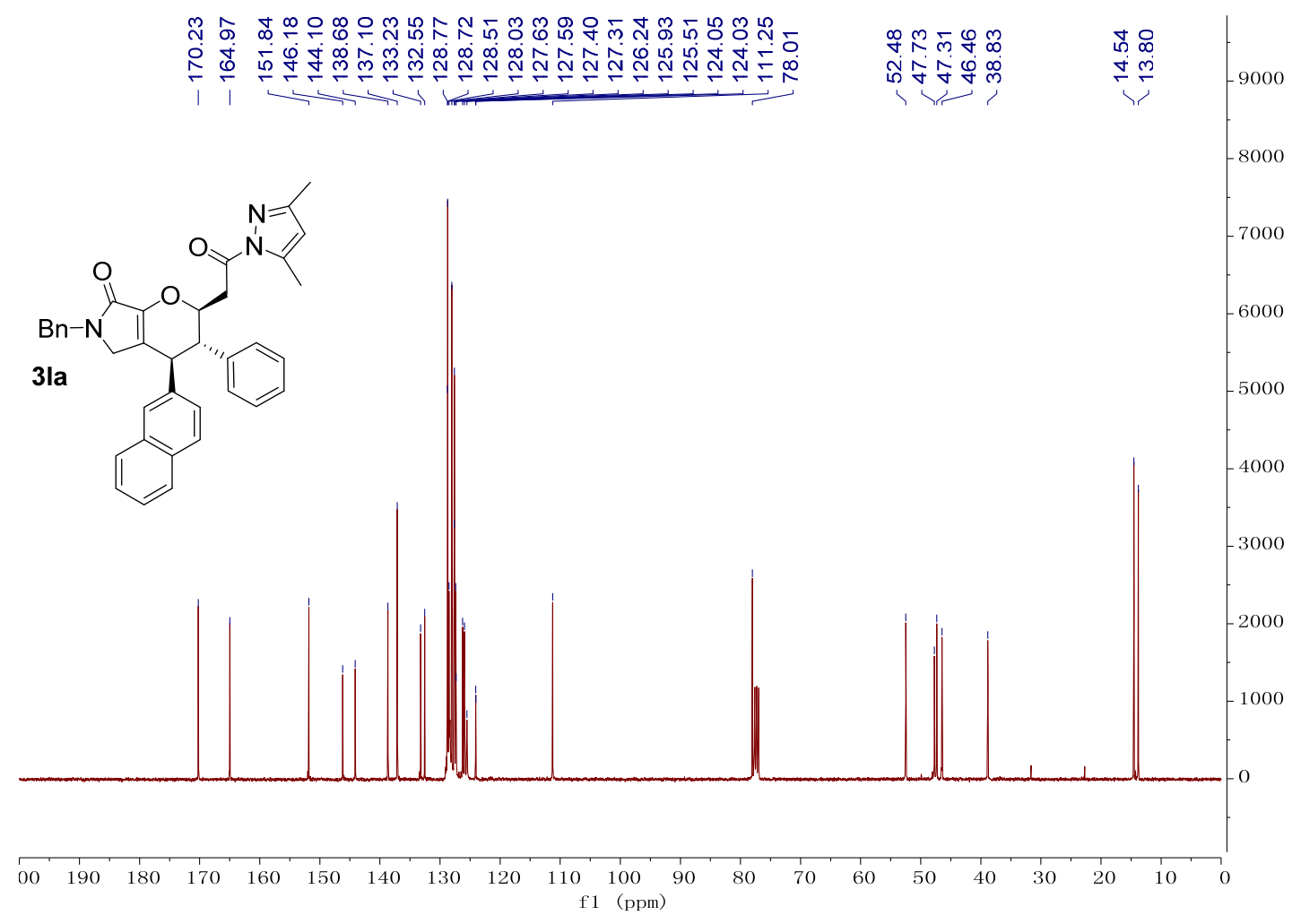



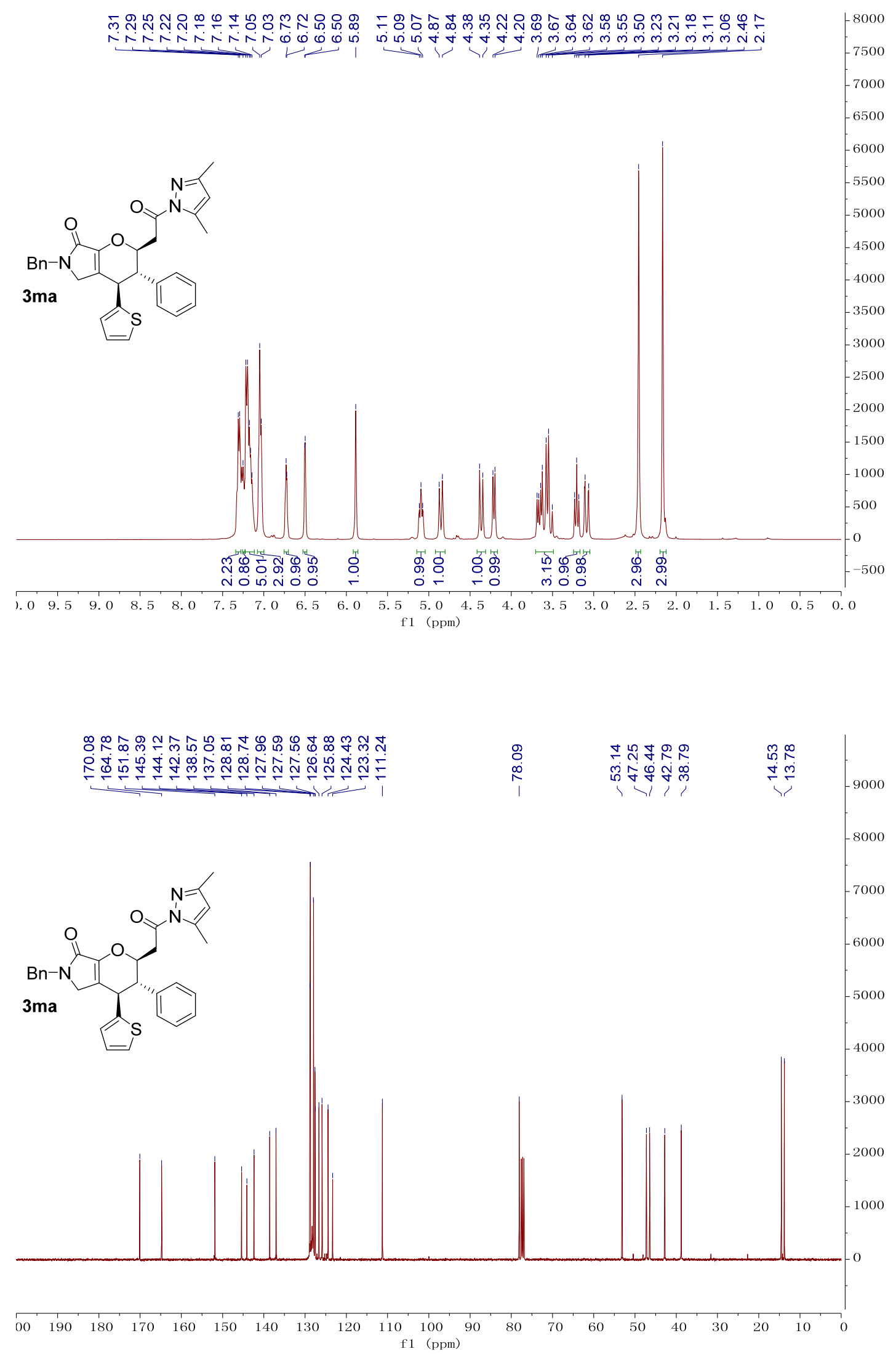

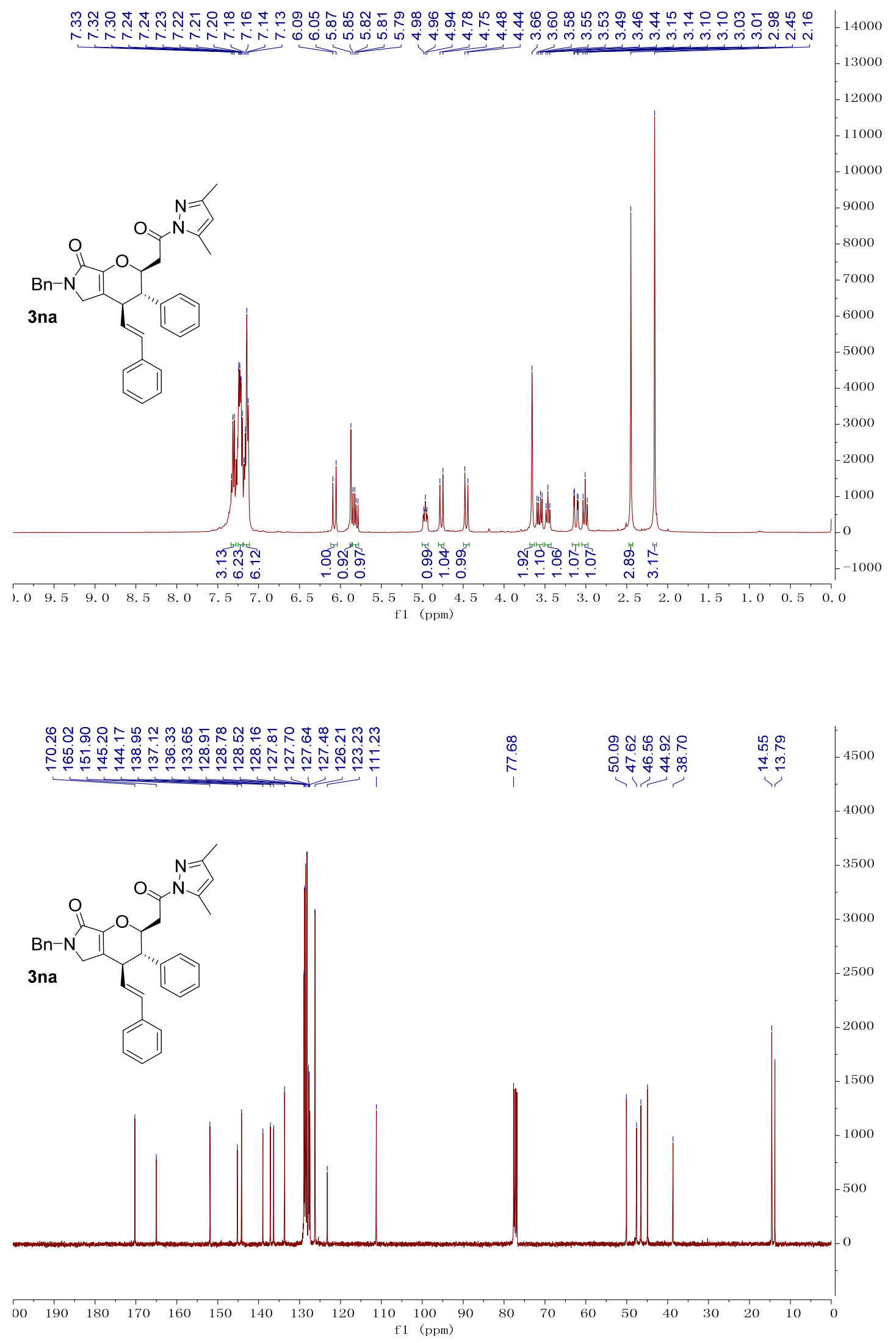


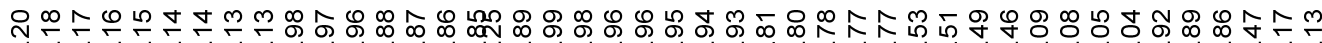

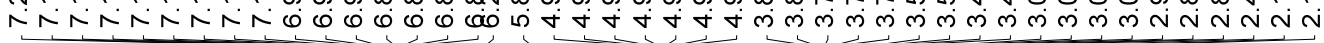<smiles>COC(=O)C1=C[C@H](c2ccccc2)[C@@H](CC(=O)n2nc(C)cc2C)O[C@H]1c1ccccc1</smiles>

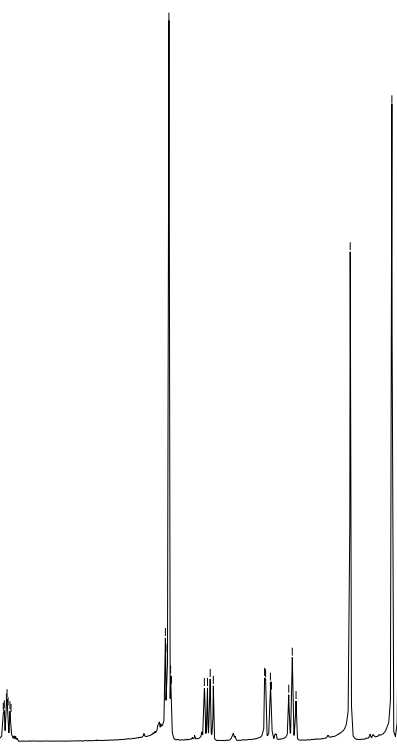

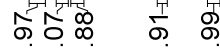
范

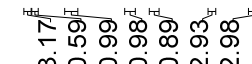

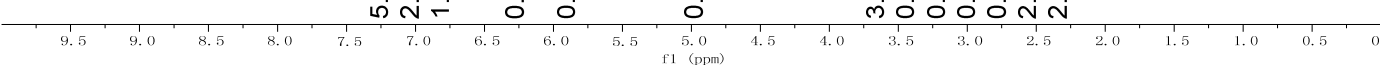

@ :

임

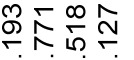

กิ่ธน์

立倠

再市<smiles>COC(=O)C1=C[C@H](c2ccccc2)[C@@H](CC(=O)n2nc(C)cc2C)O[C@H]1c1ccccc1</smiles>
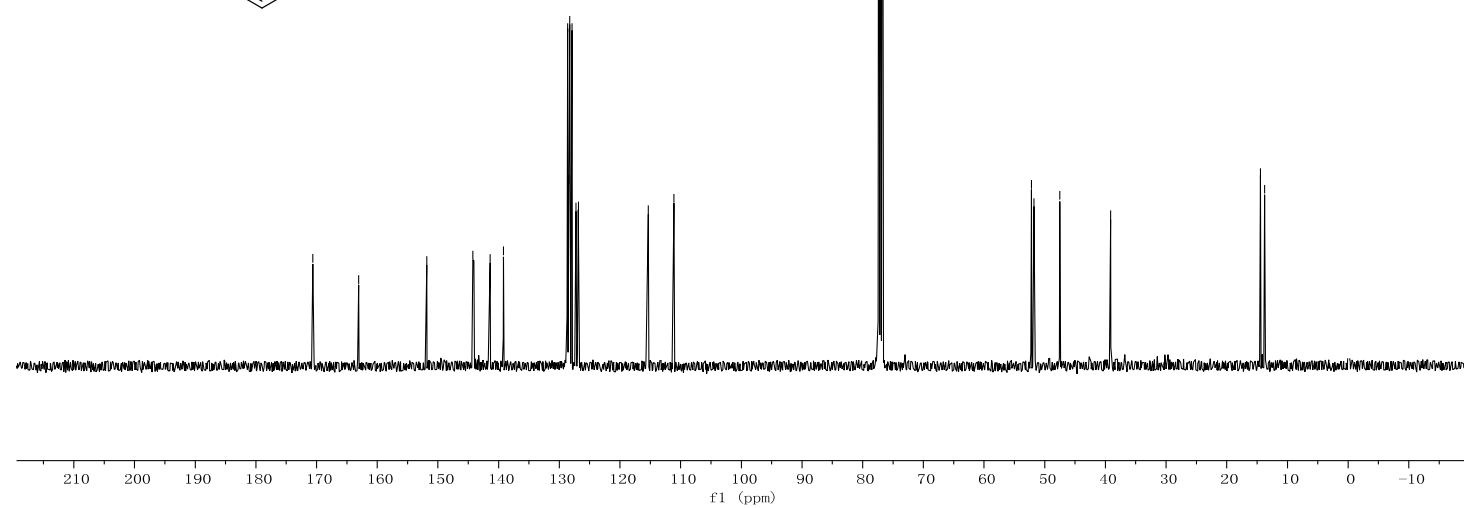


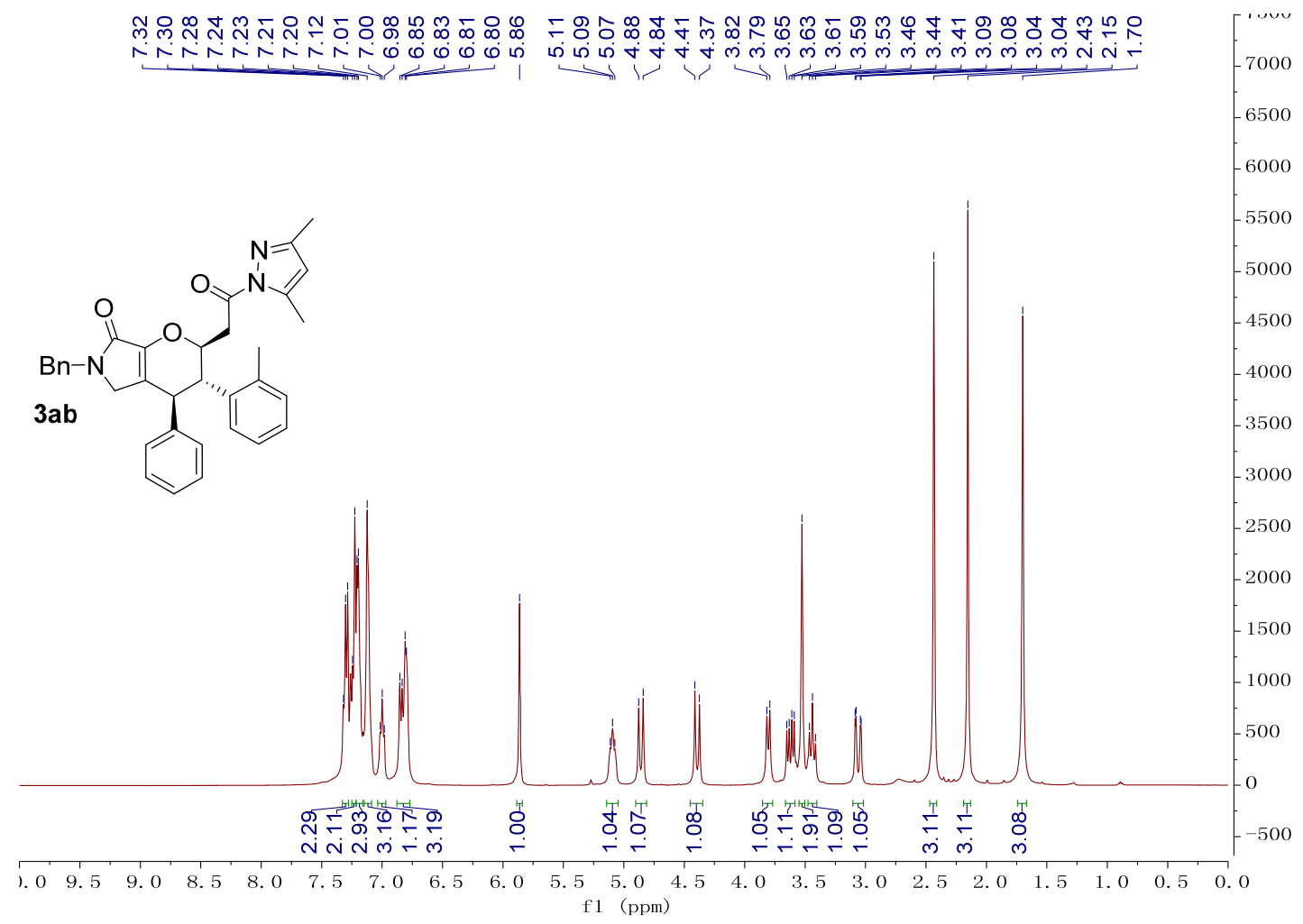

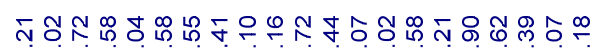

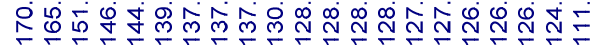

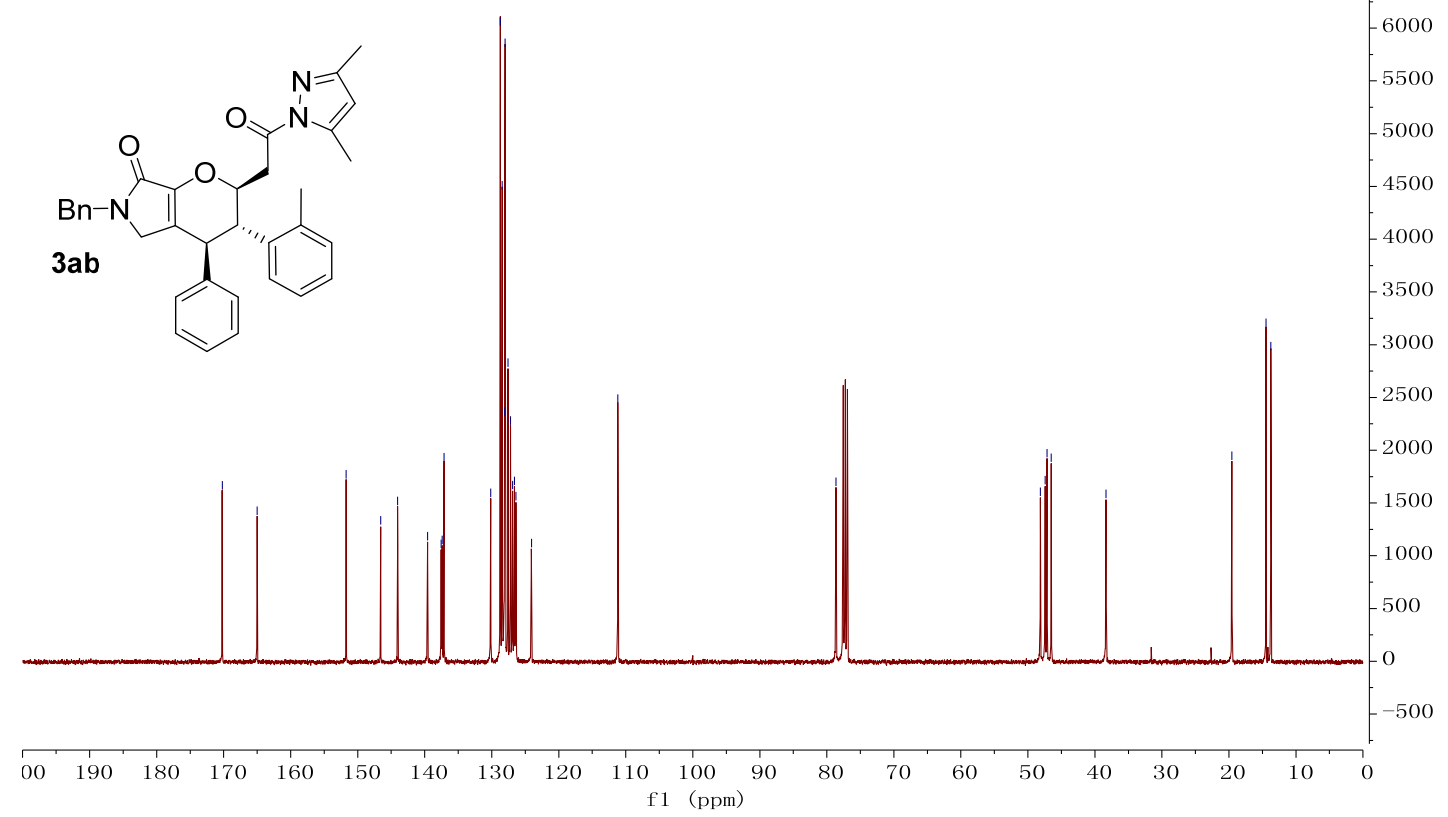




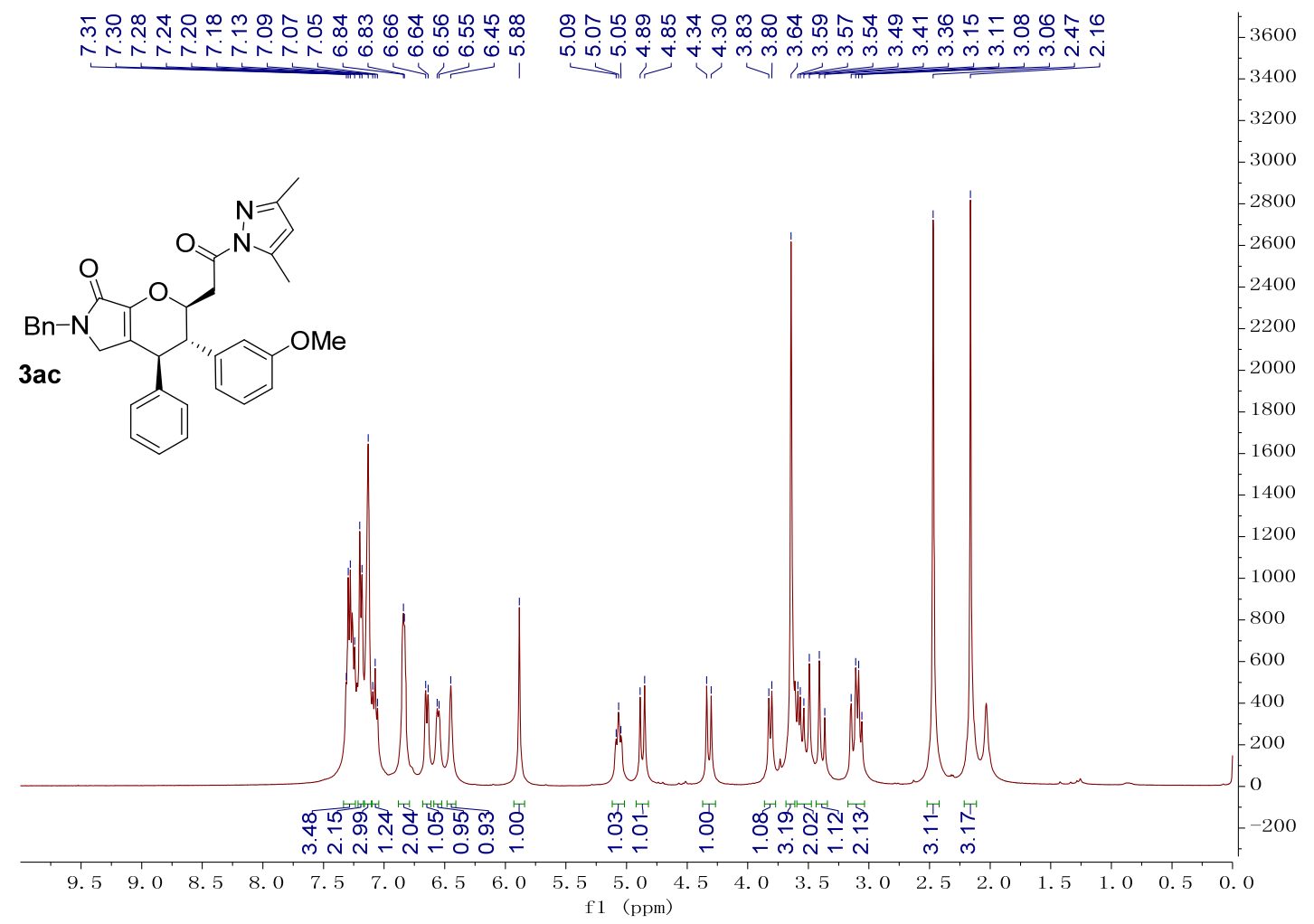

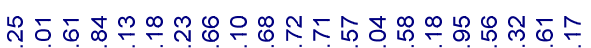

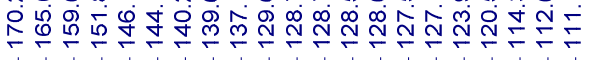

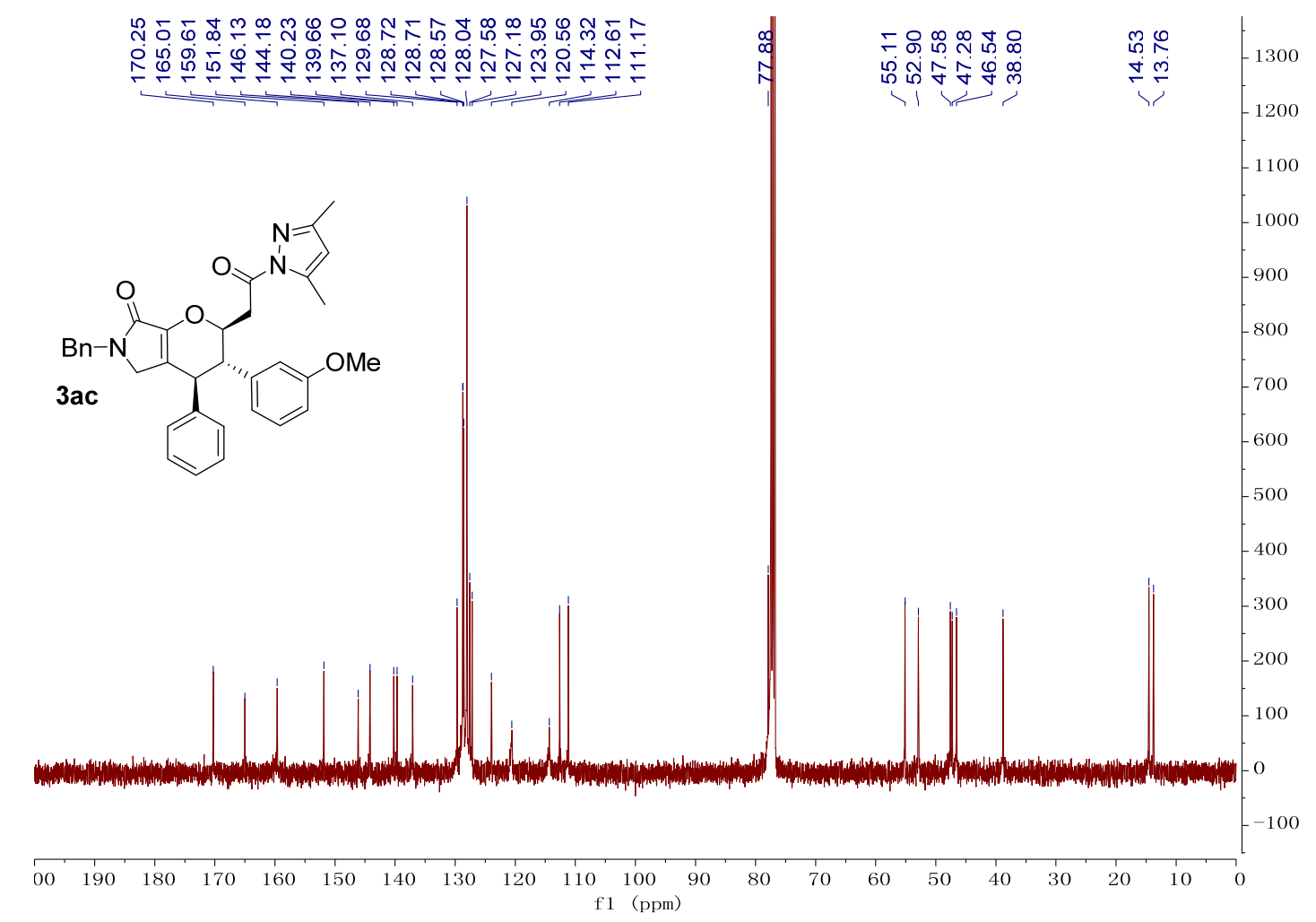



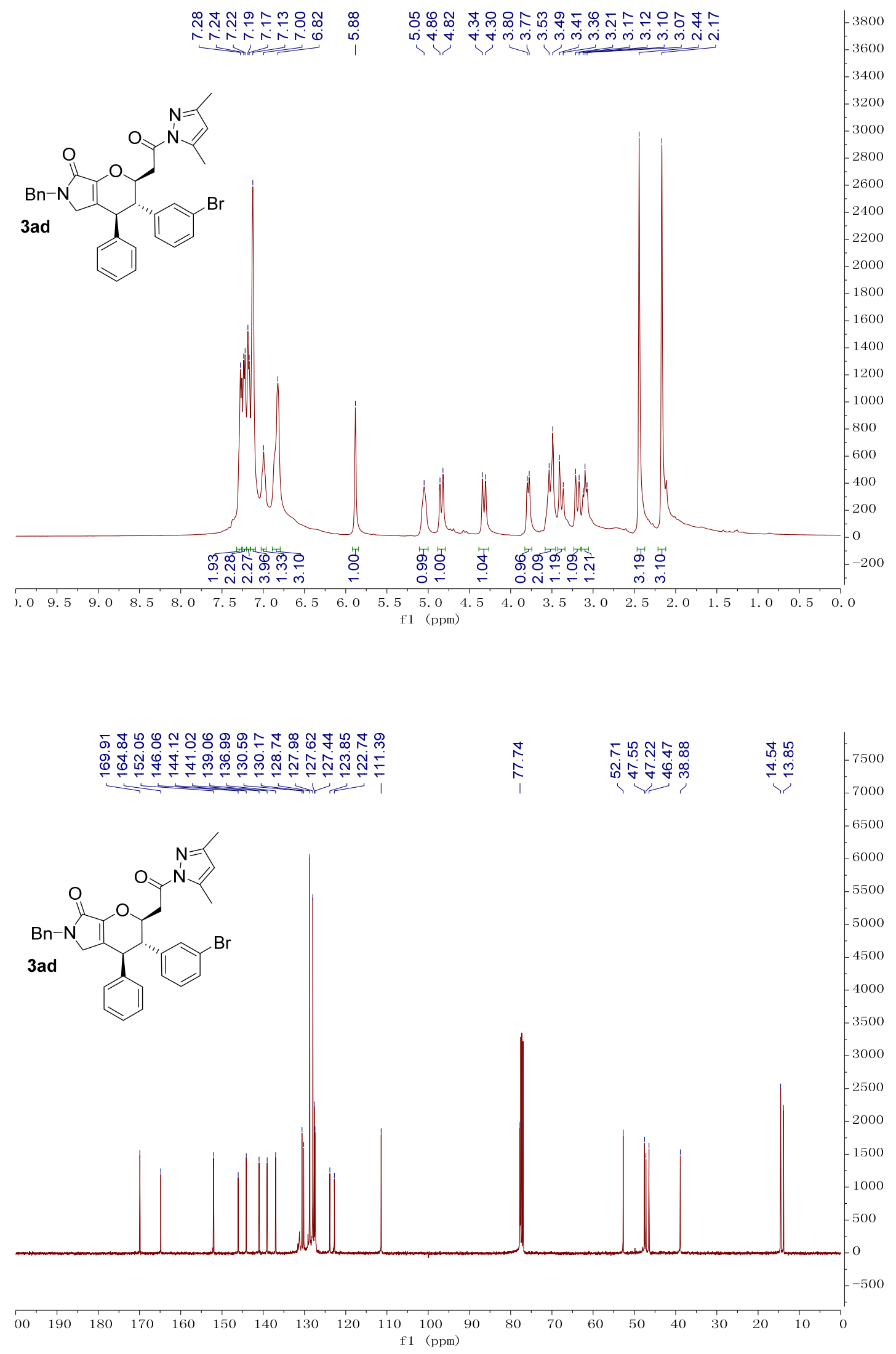

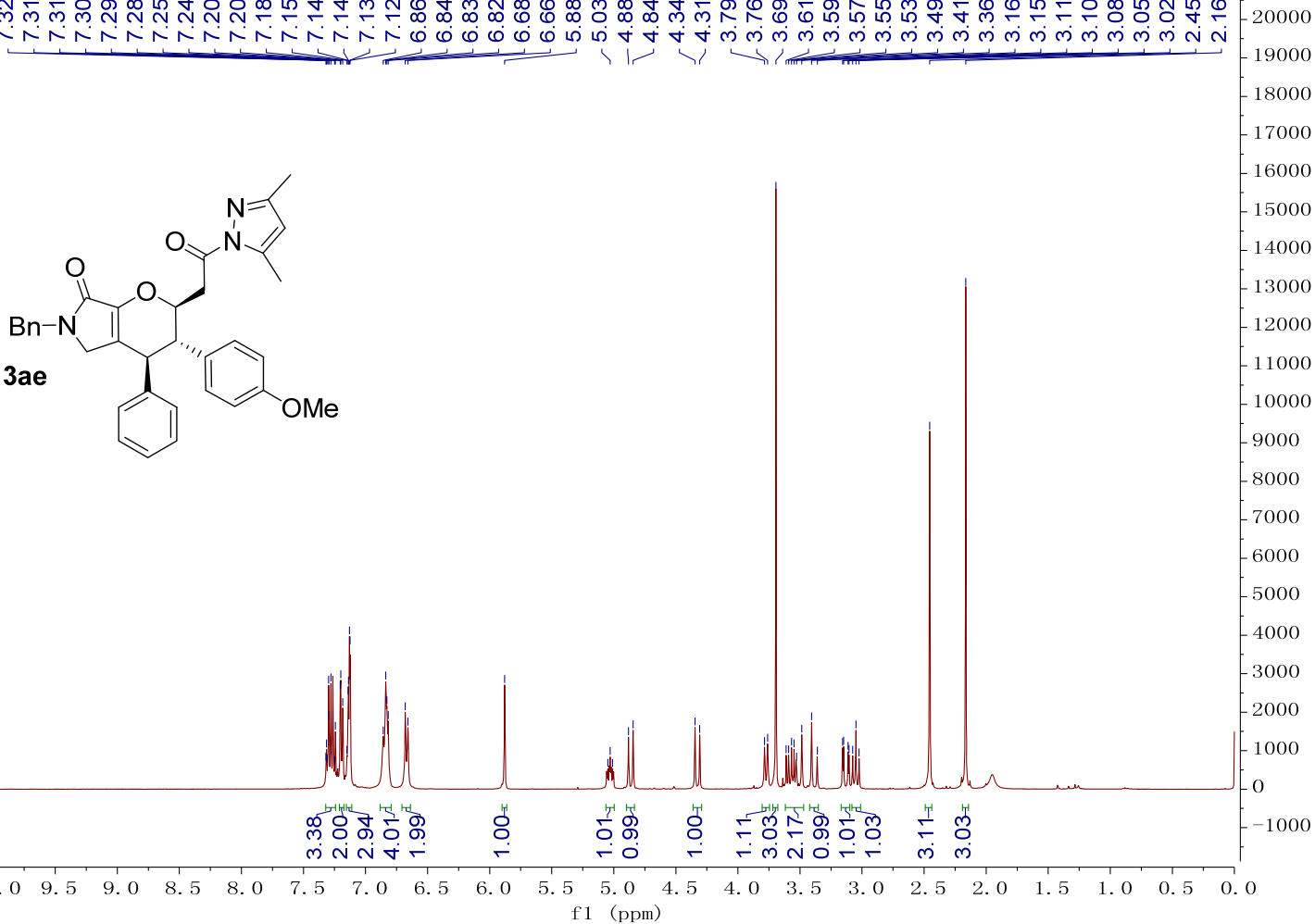

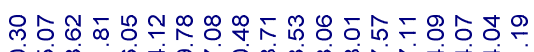

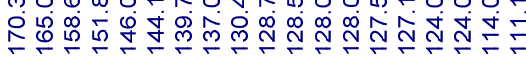

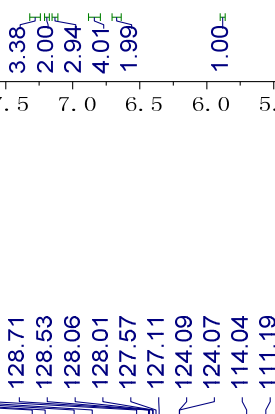

1 (

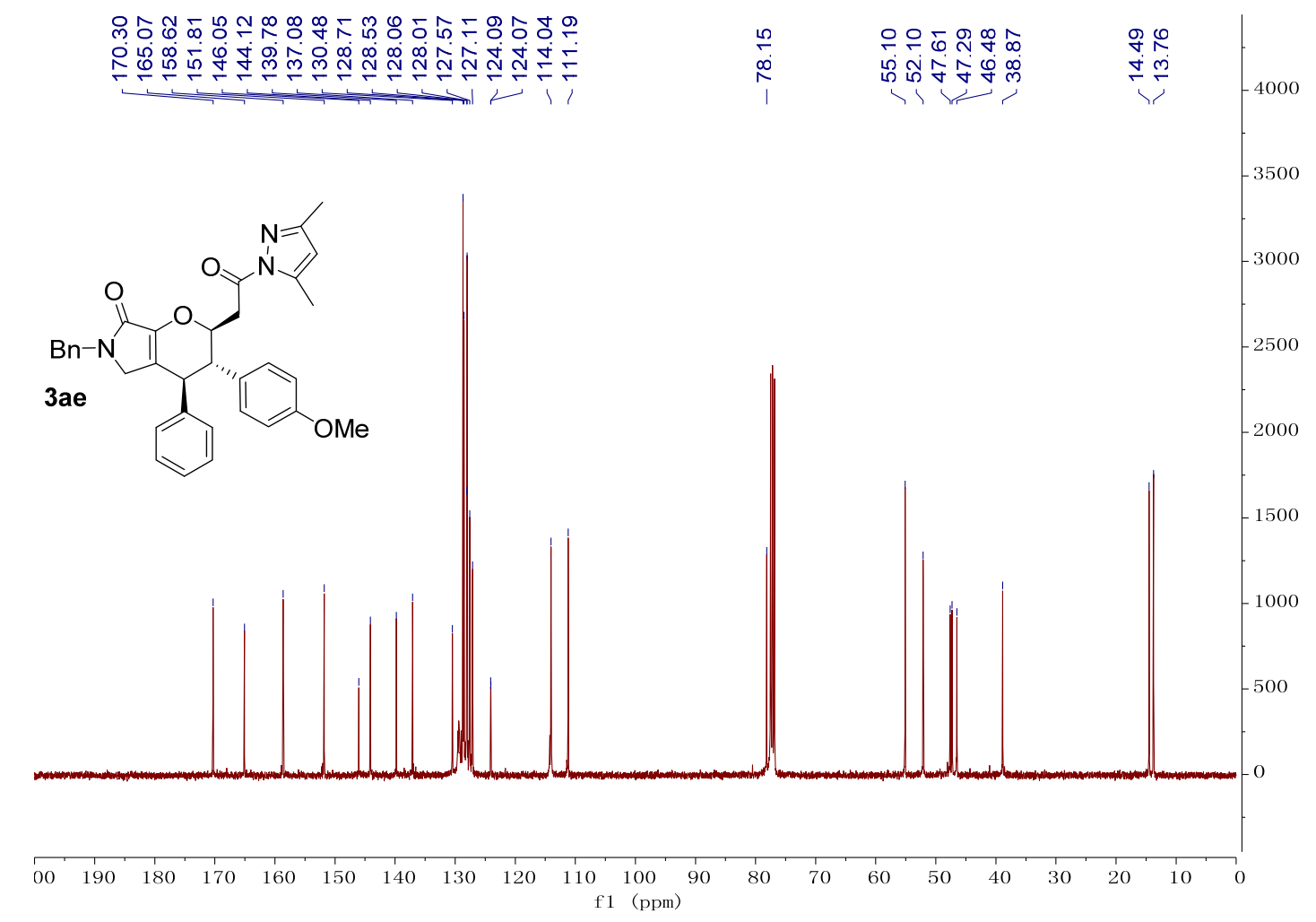



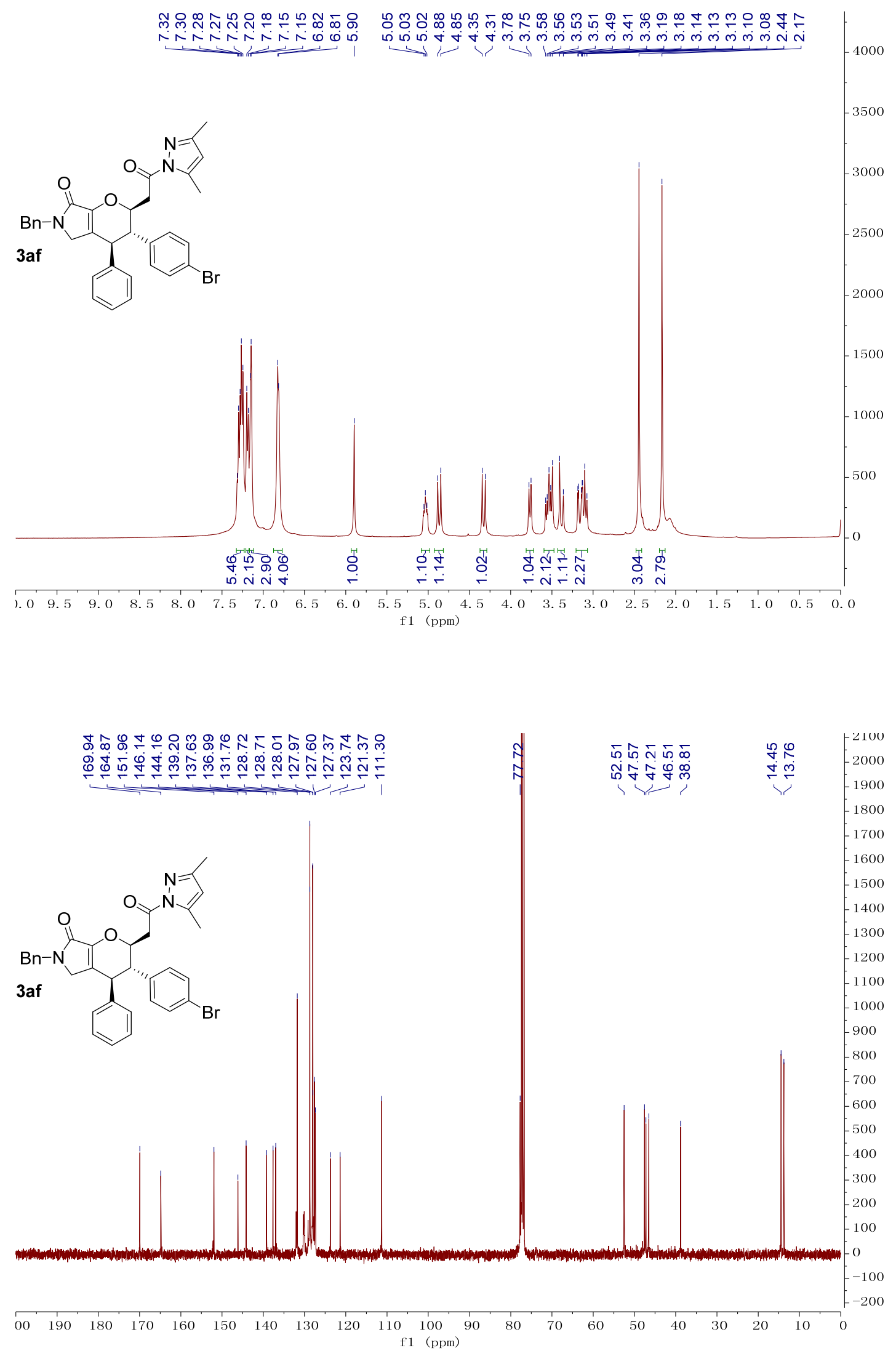

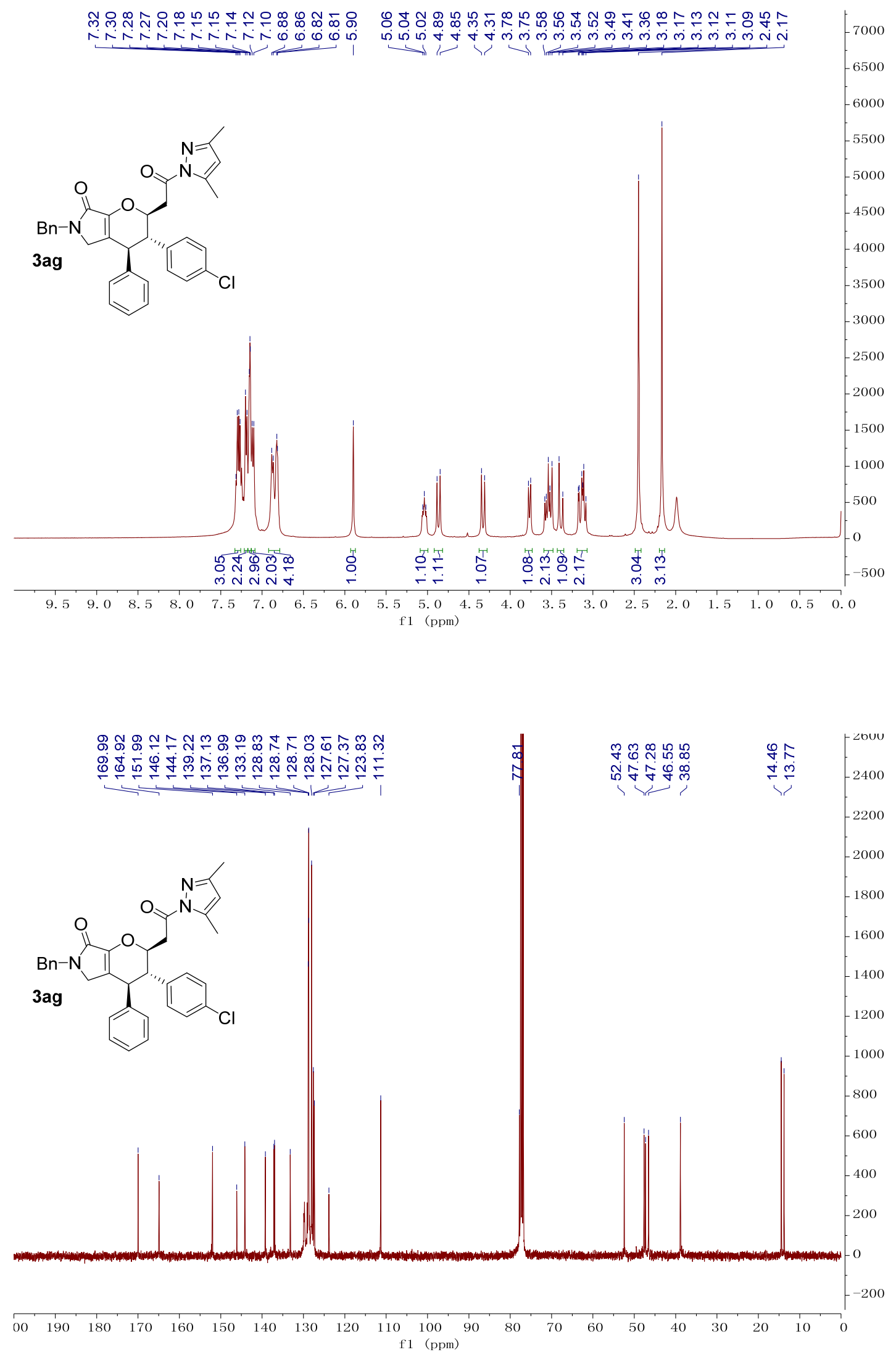


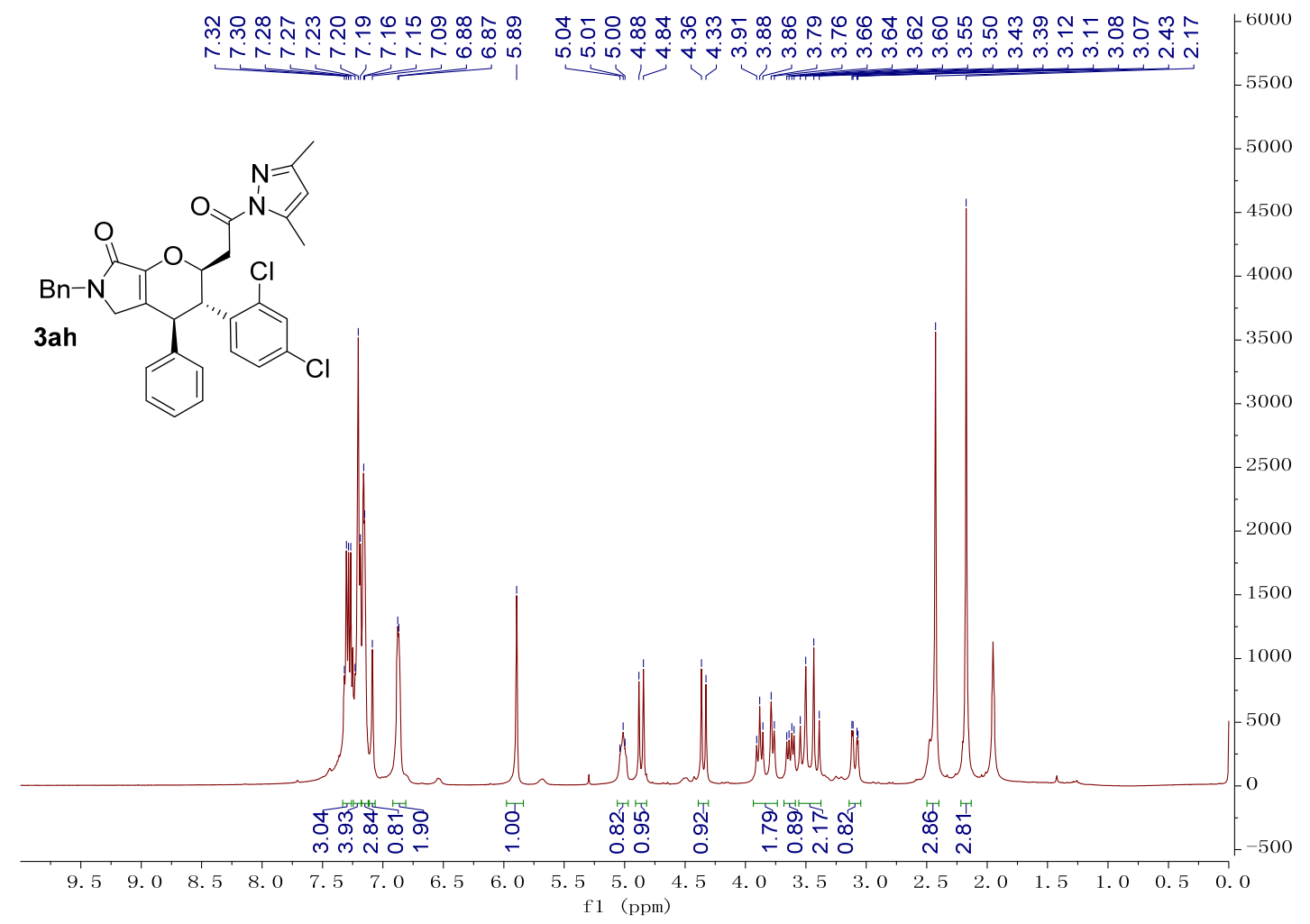

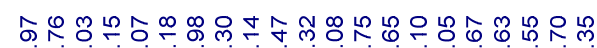

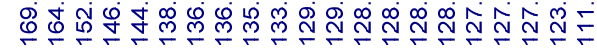

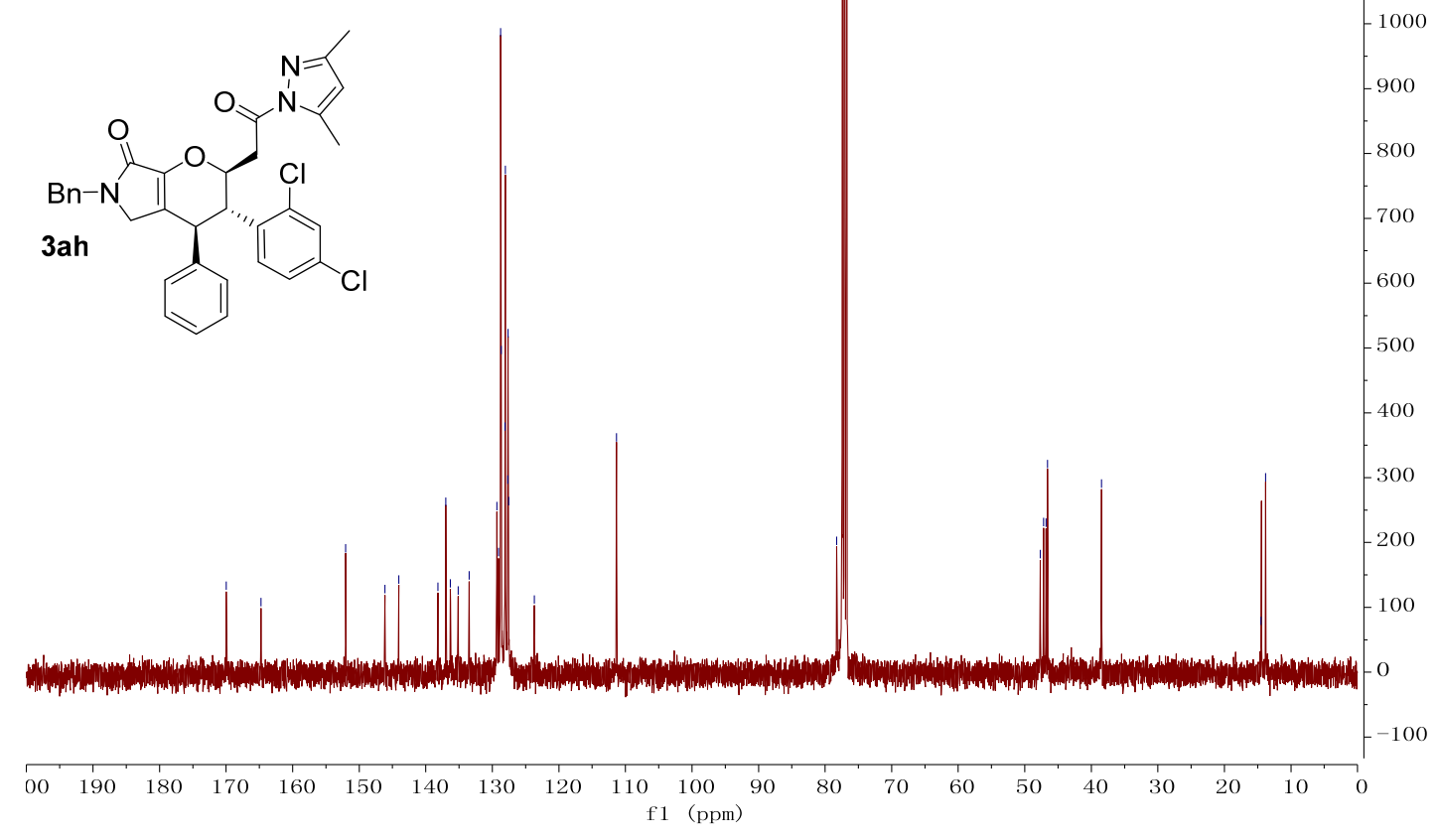


<smiles>Cc1cc(C)n(C(=O)C[C@@H]2OC3=C(CN(Cc4ccccc4)C3=O)[C@@H](c3ccccc3)[C@H]2c2ccc3ccccc3c2)n1</smiles>

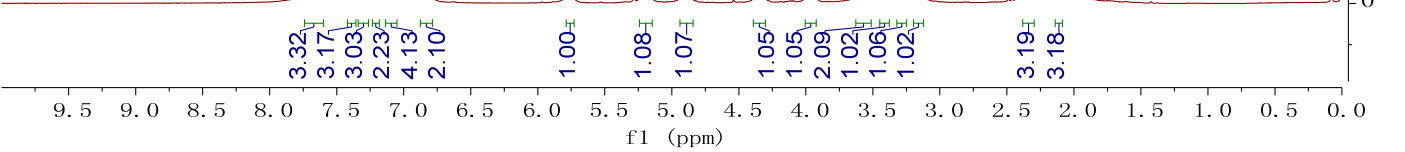

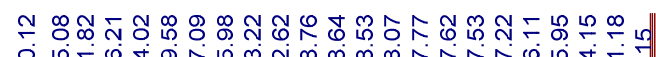

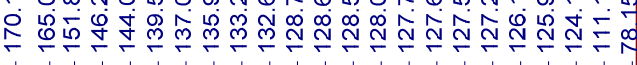

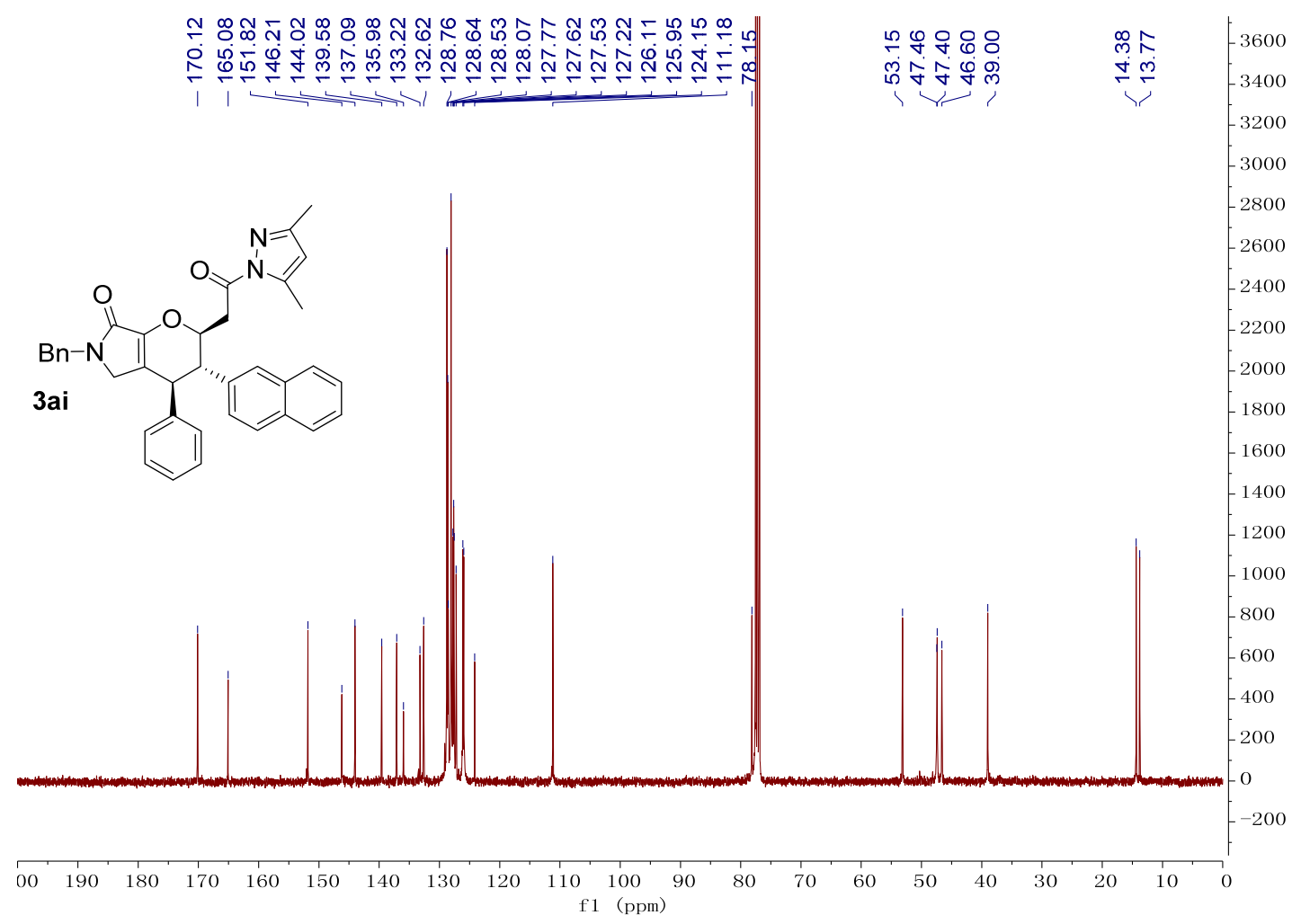



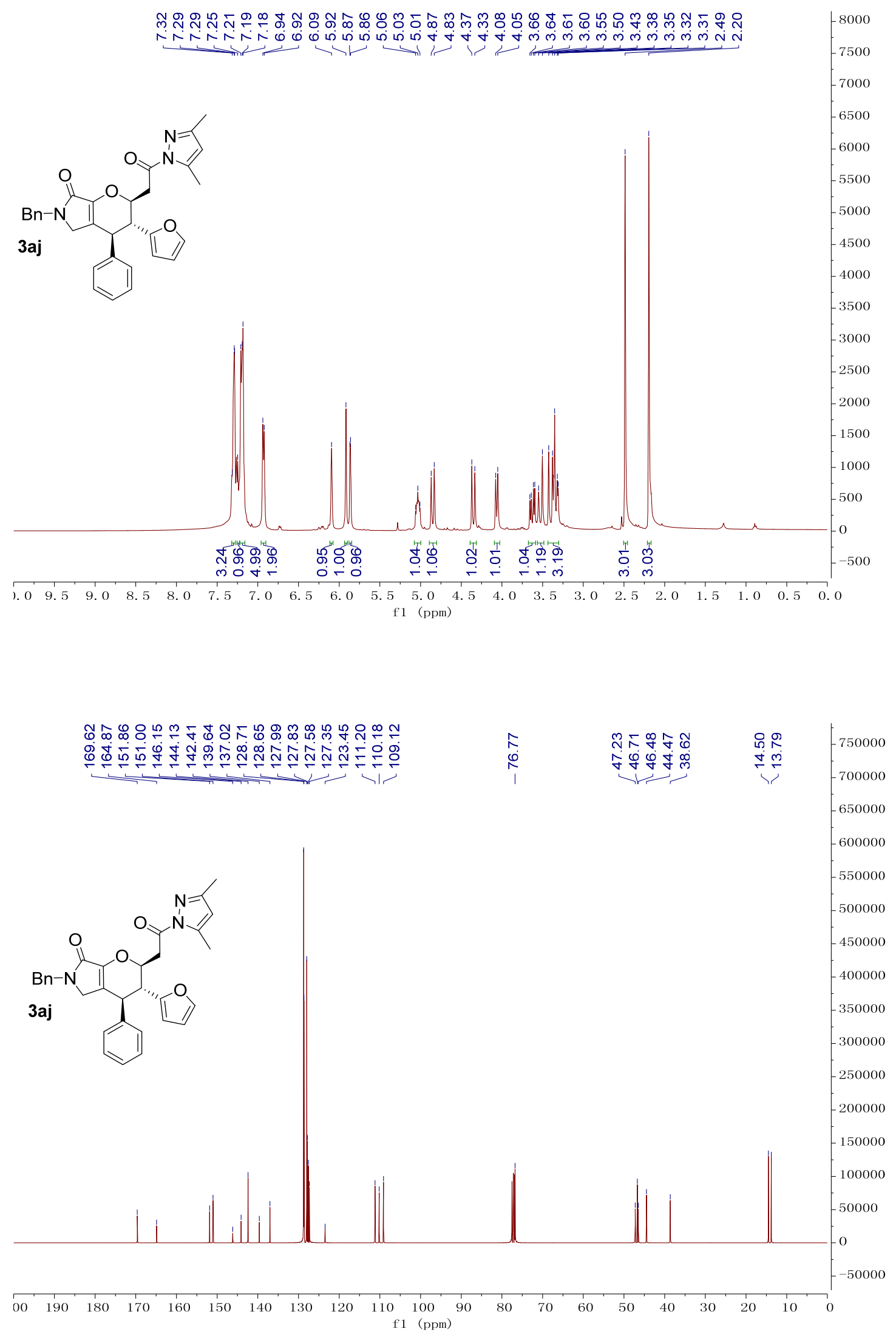


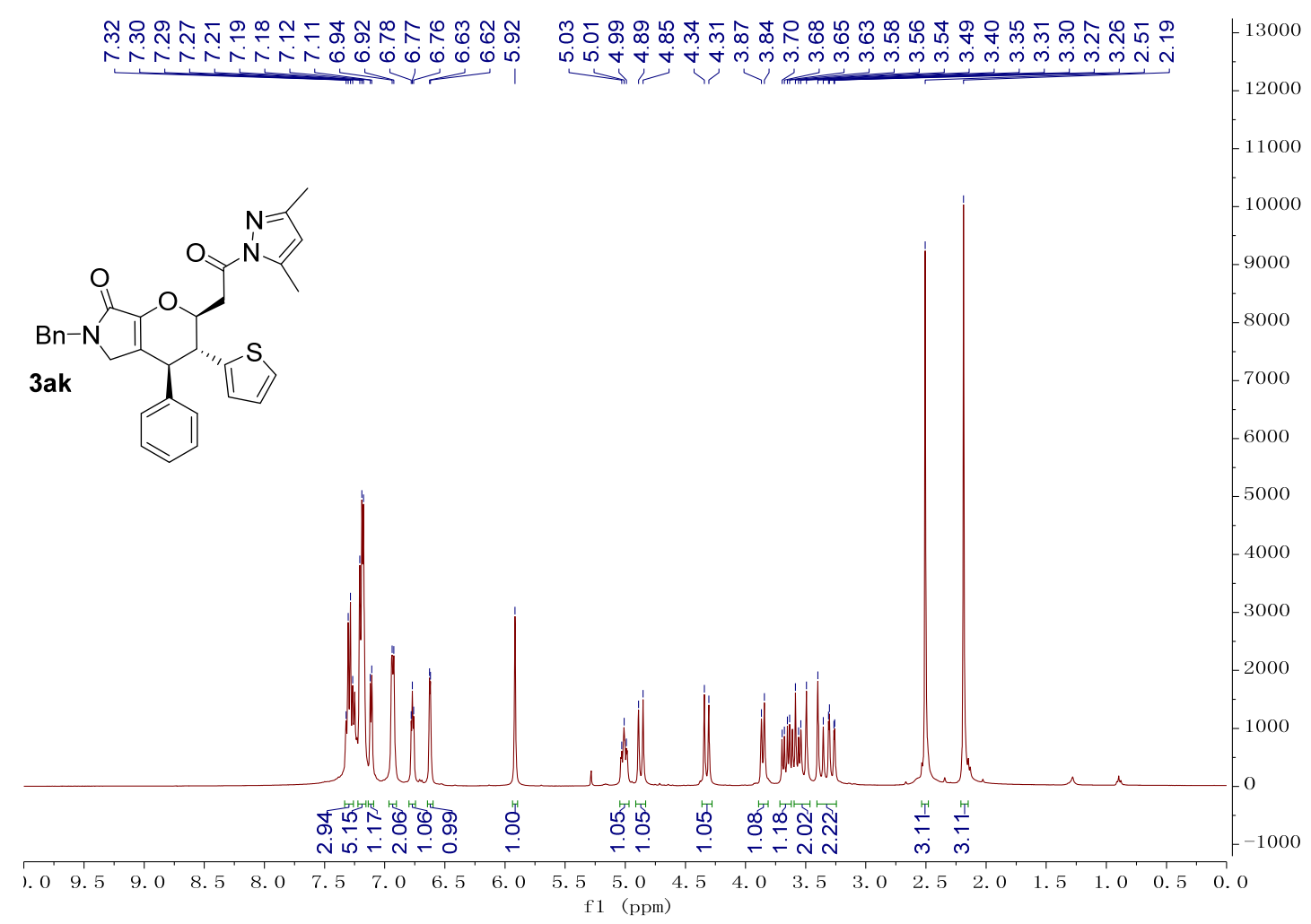

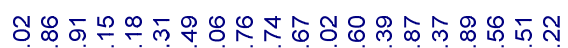

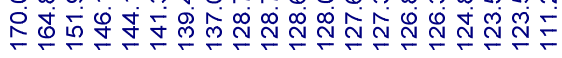

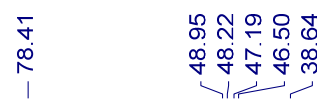

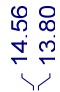

12000000 11000000 10000000<smiles>Cc1cc(C)n(C(=O)CC2OC3=C(C=CC(Cc4ccccc4)N3Cc3ccccc3)C(c3cccs3)C(c3cccs3)C2=O)n1</smiles>

9000000 8000000 7000000 6000000 5000000 4000000 3000000 2000000 1000000

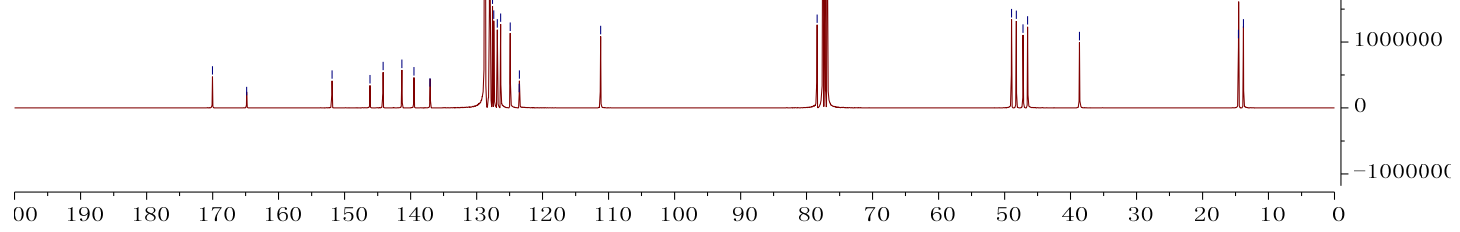




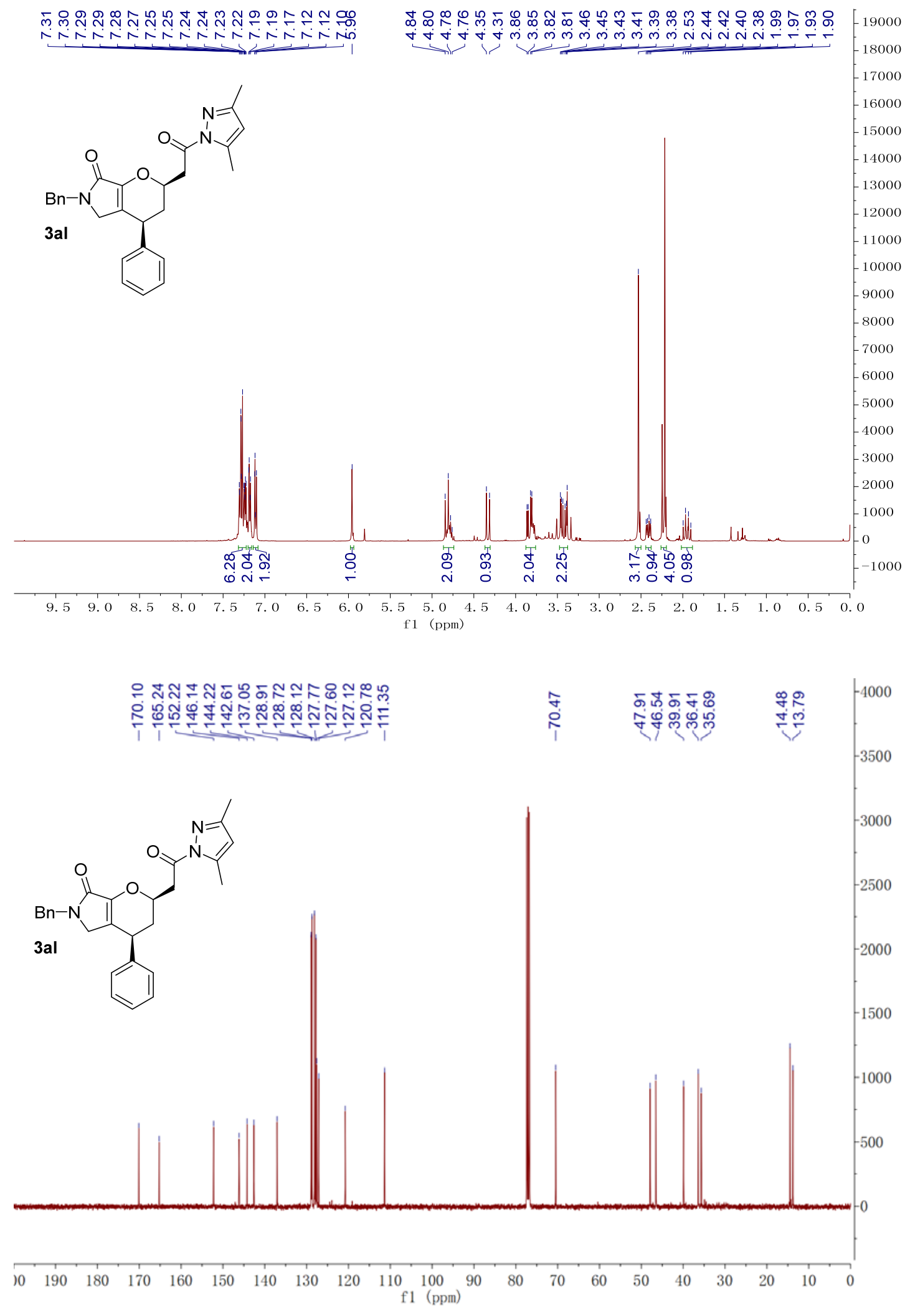




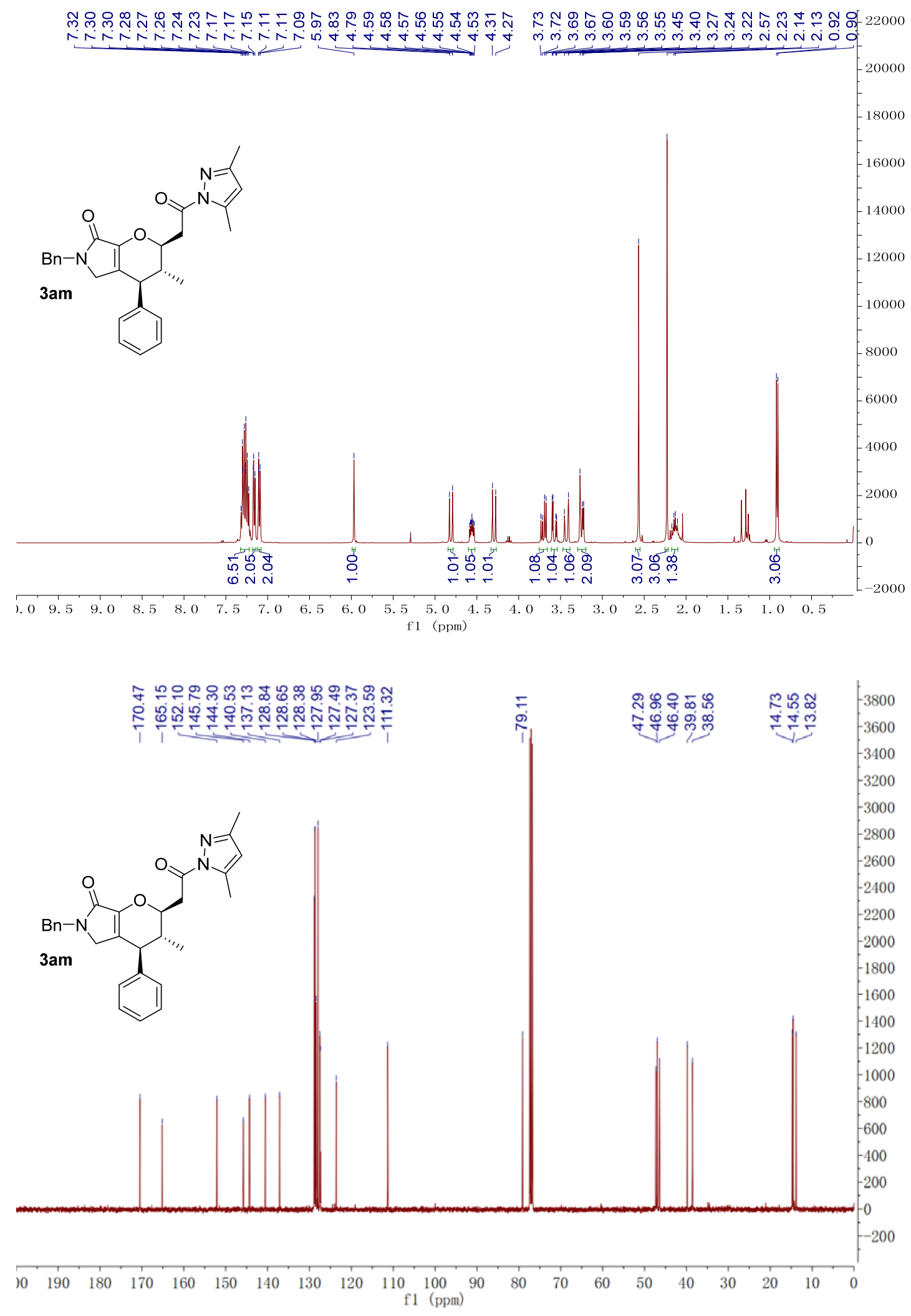




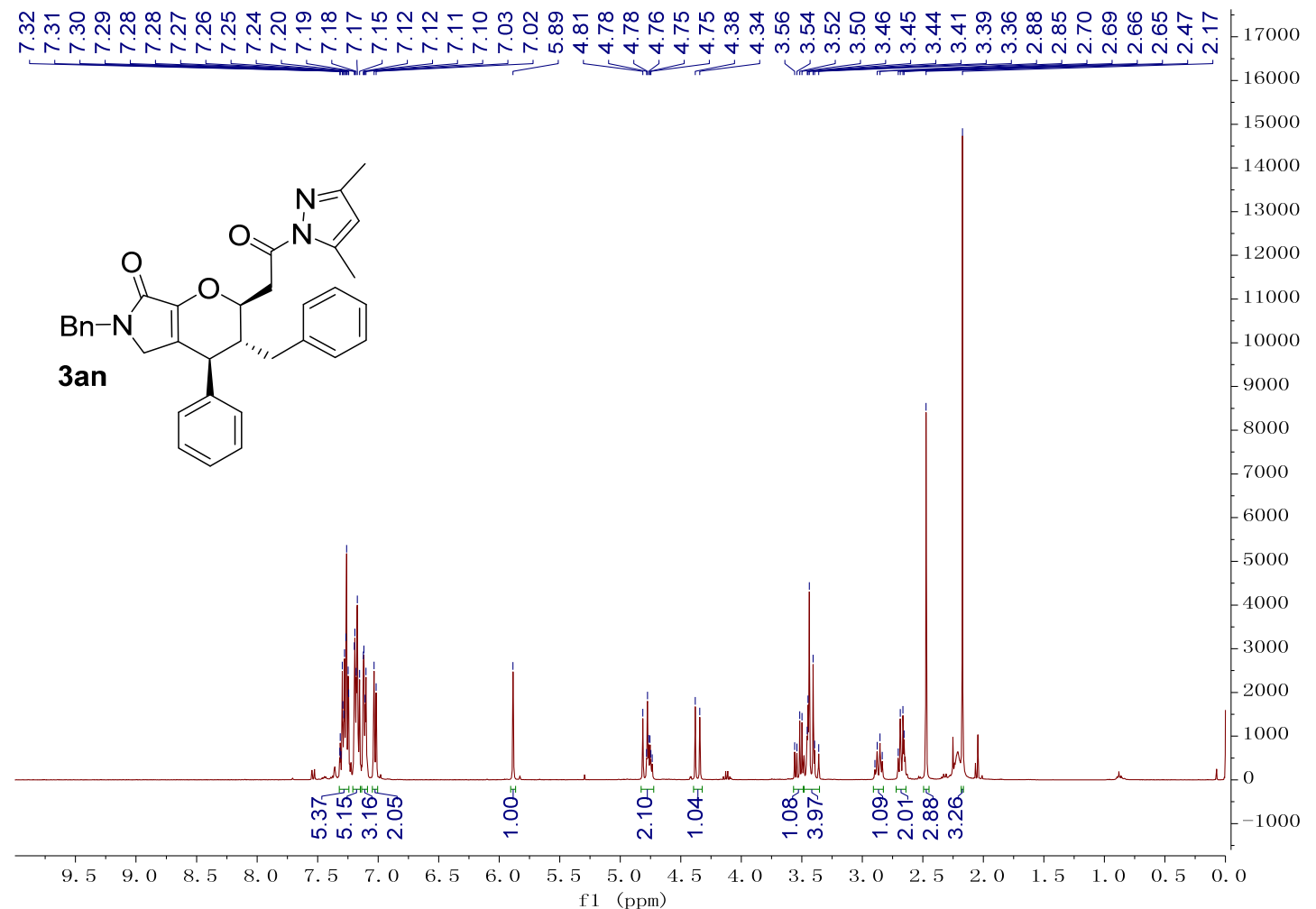

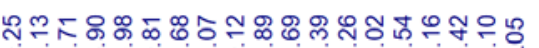
ํํำ

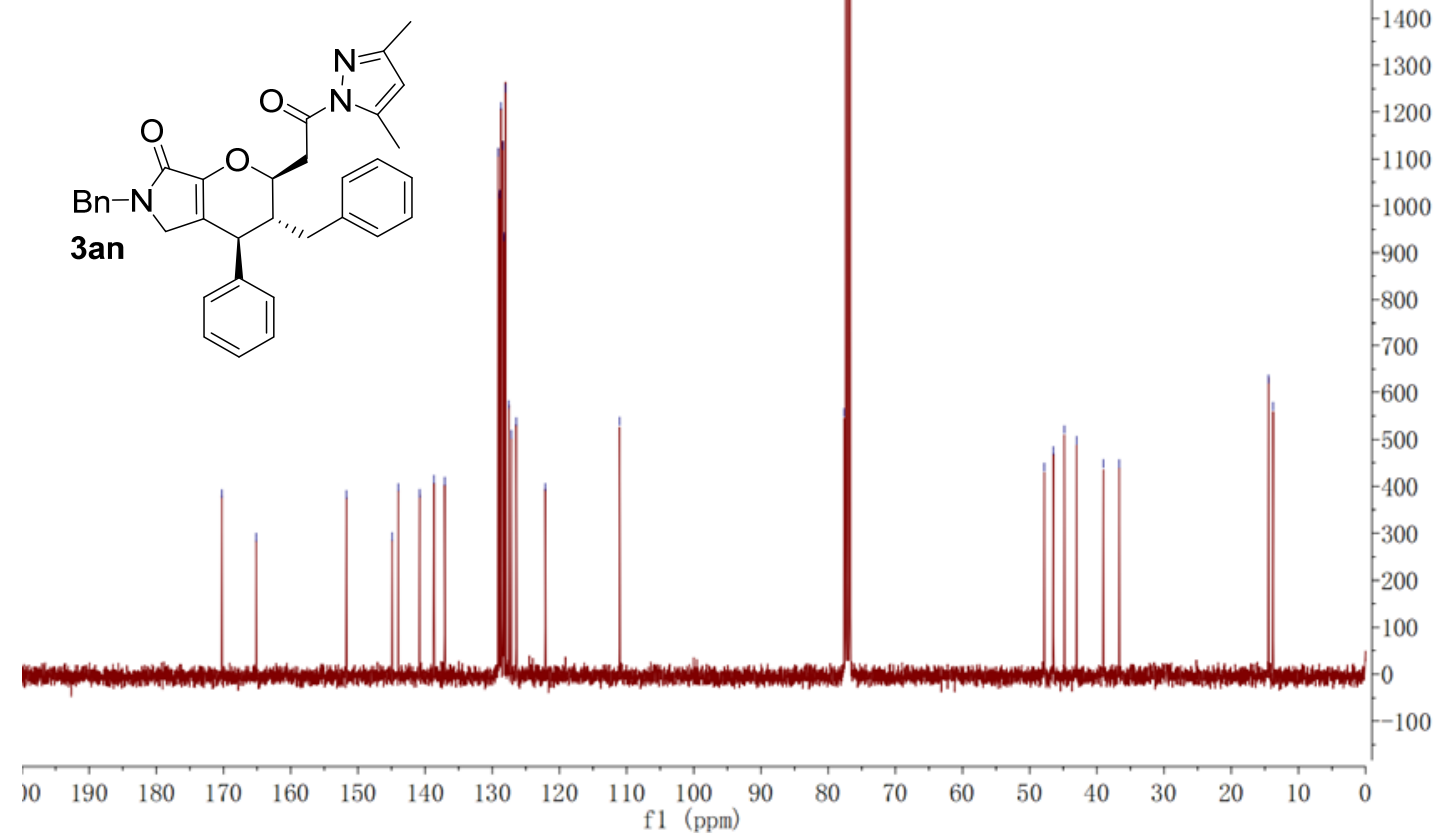



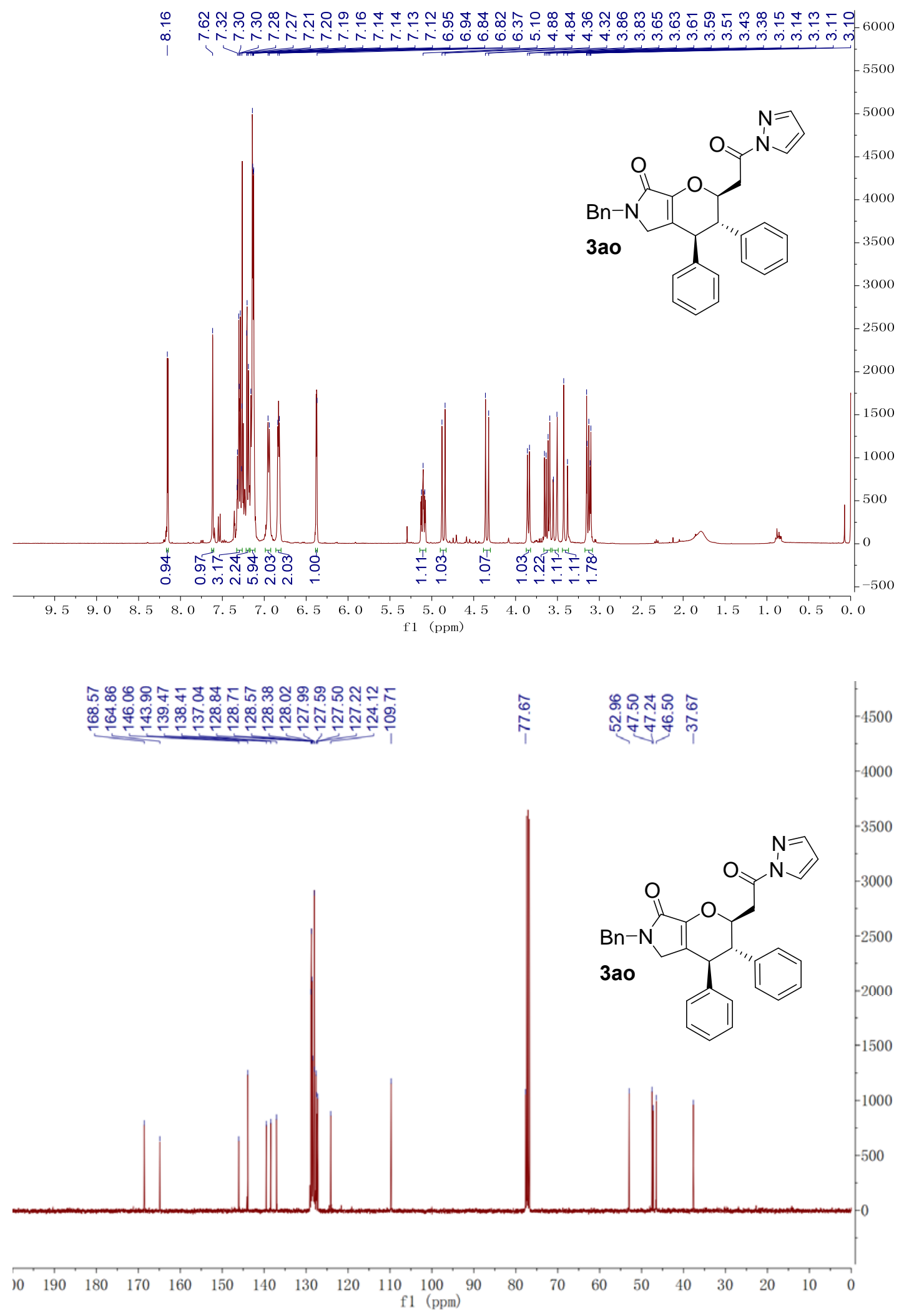

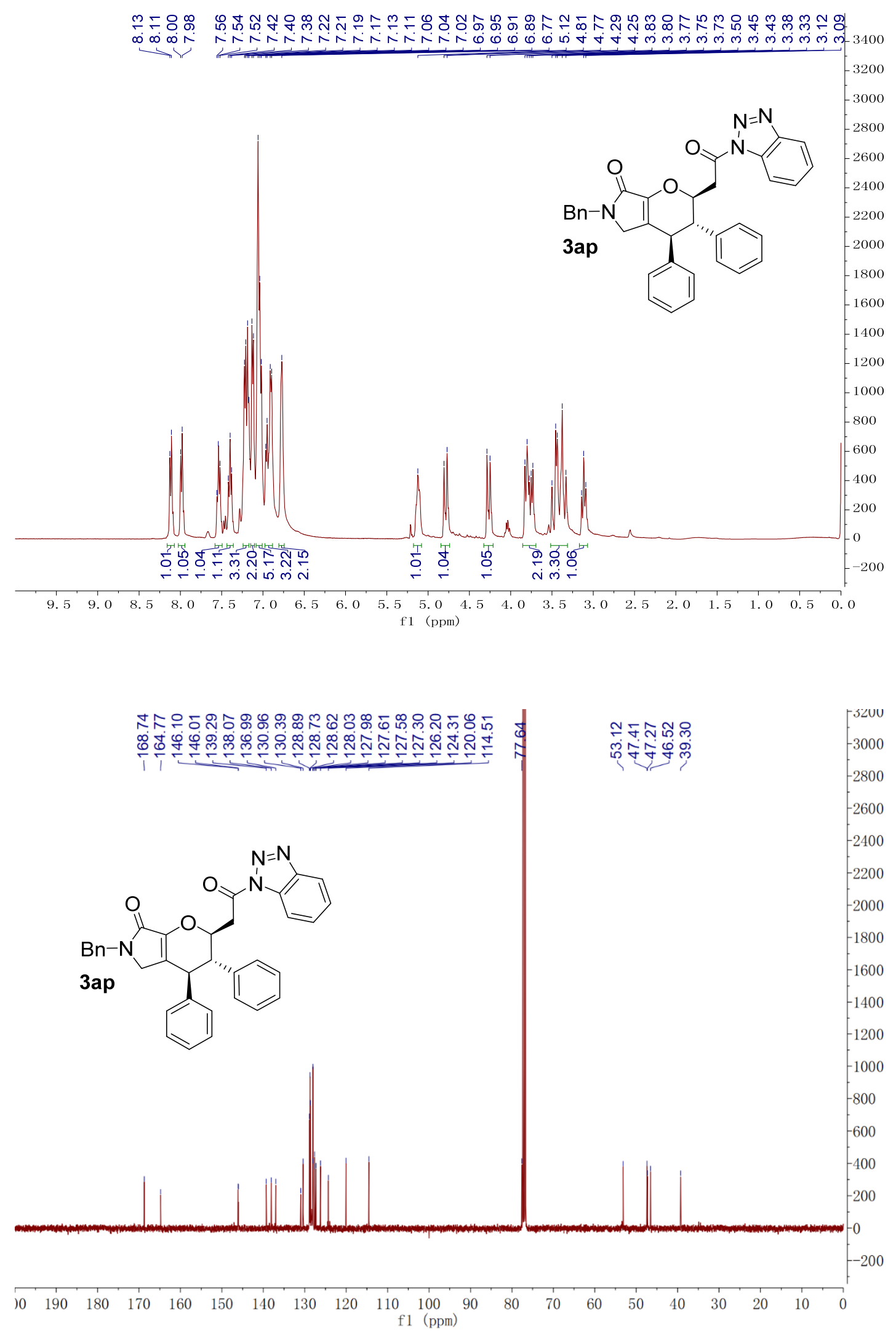

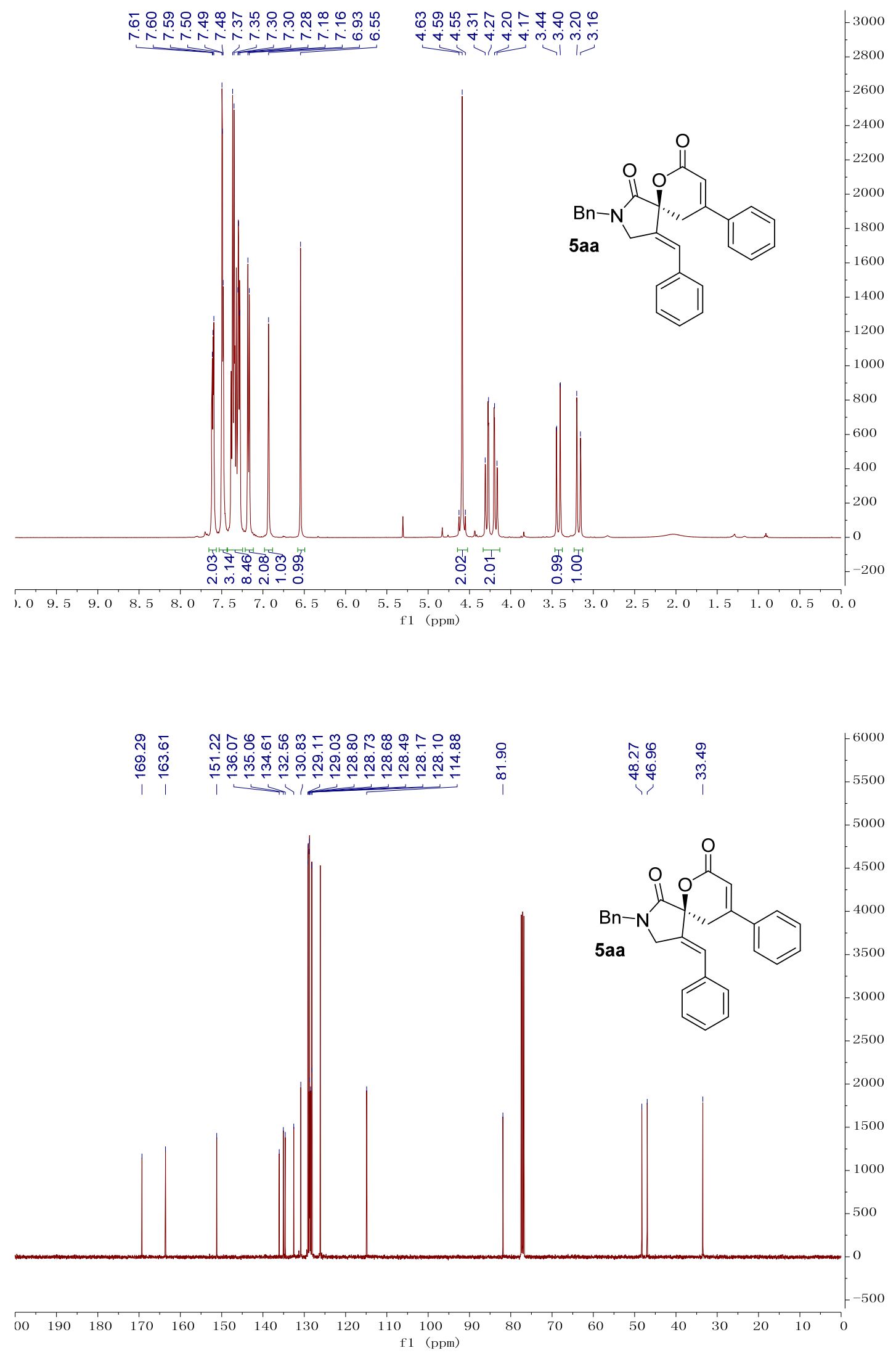

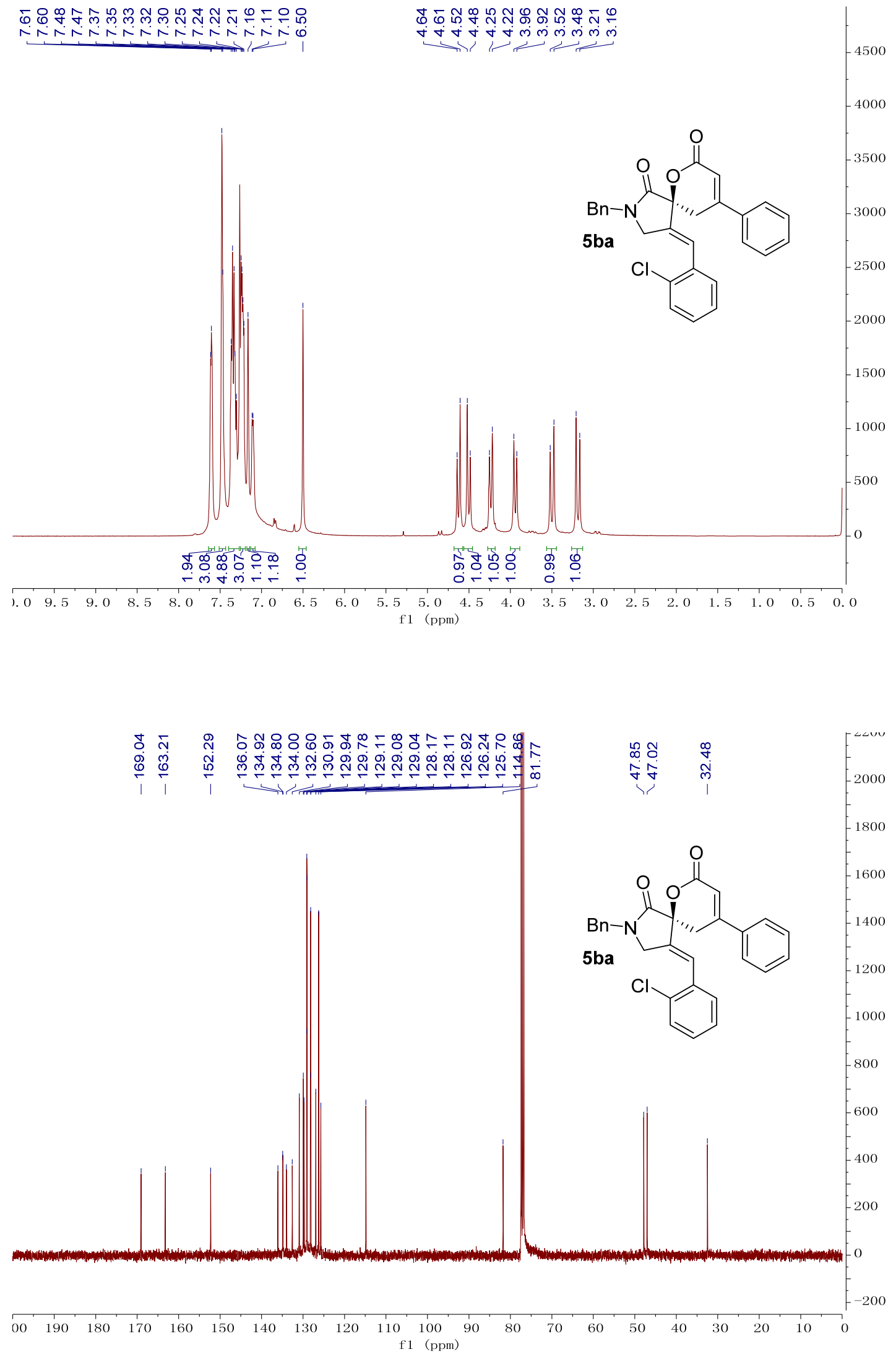


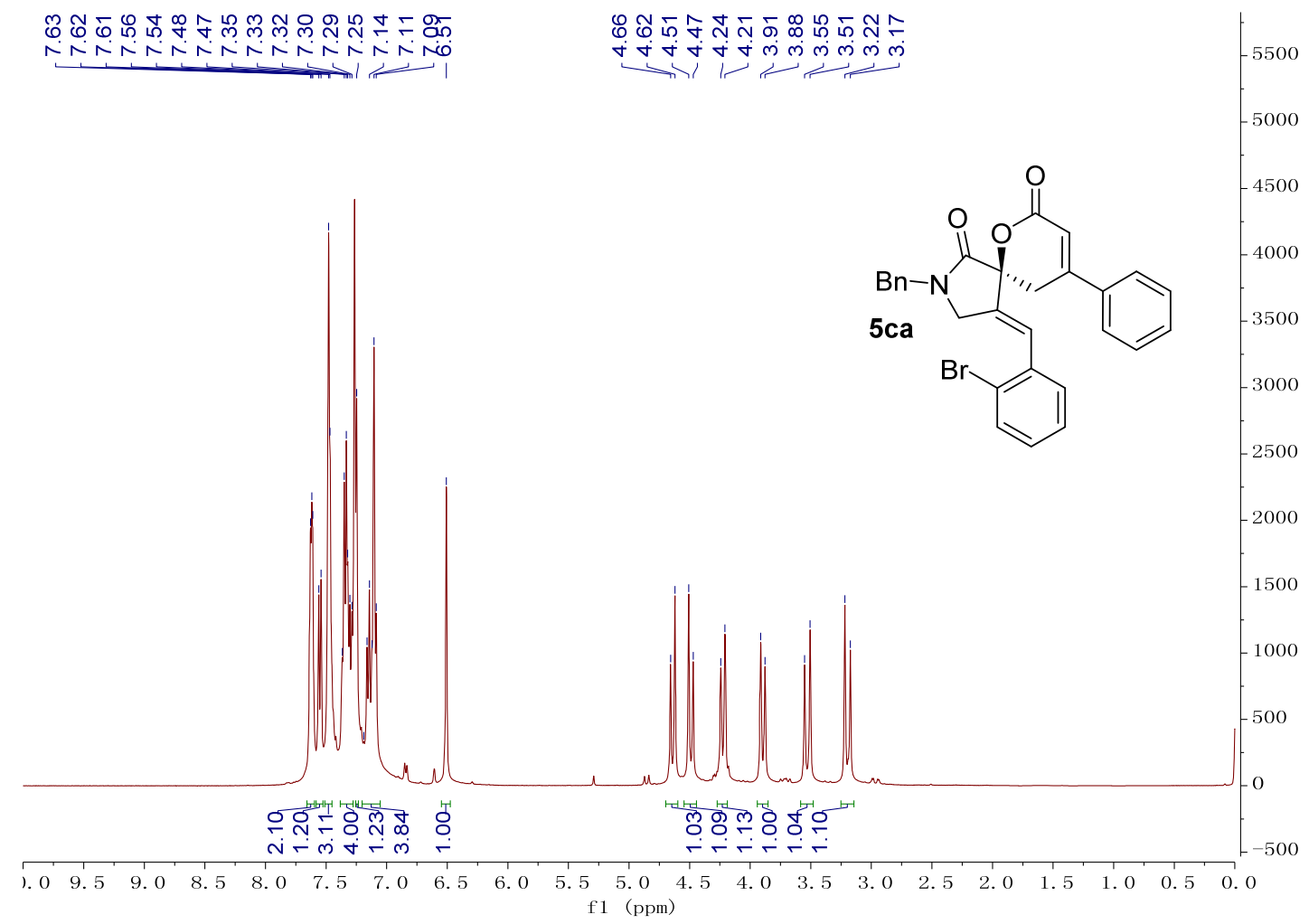

50

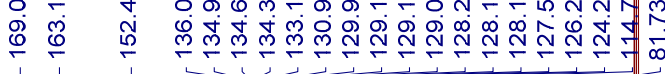

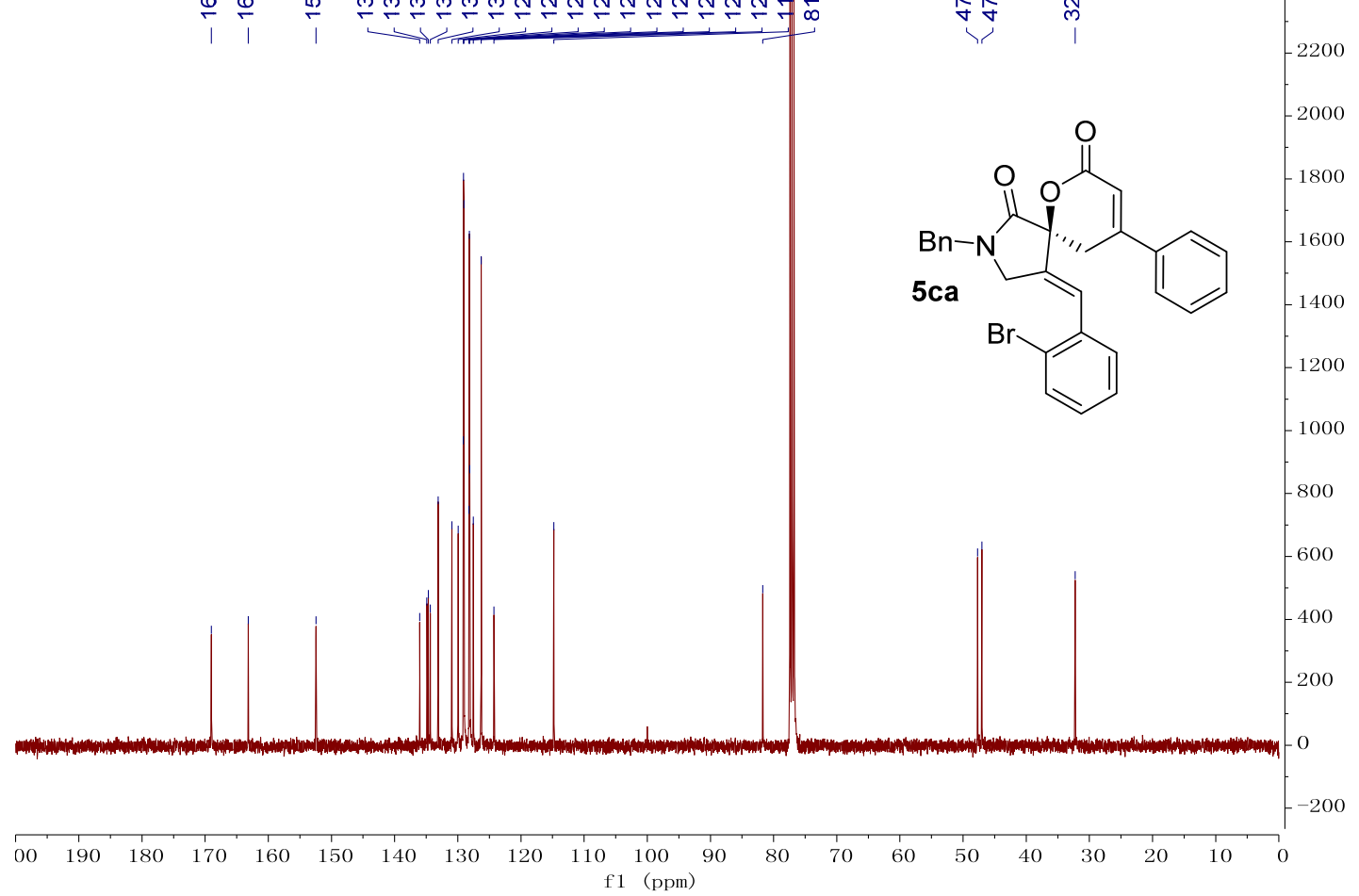



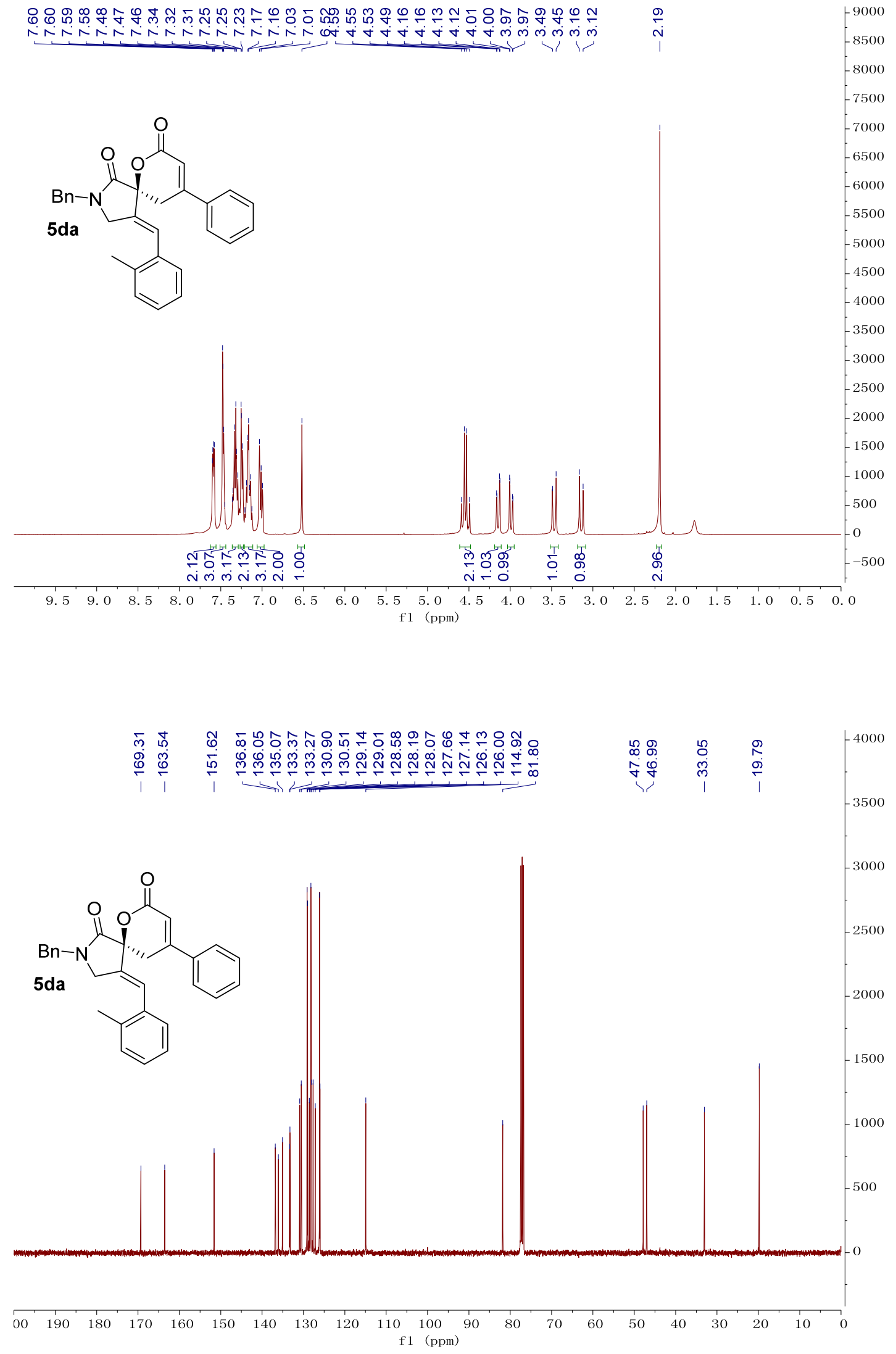

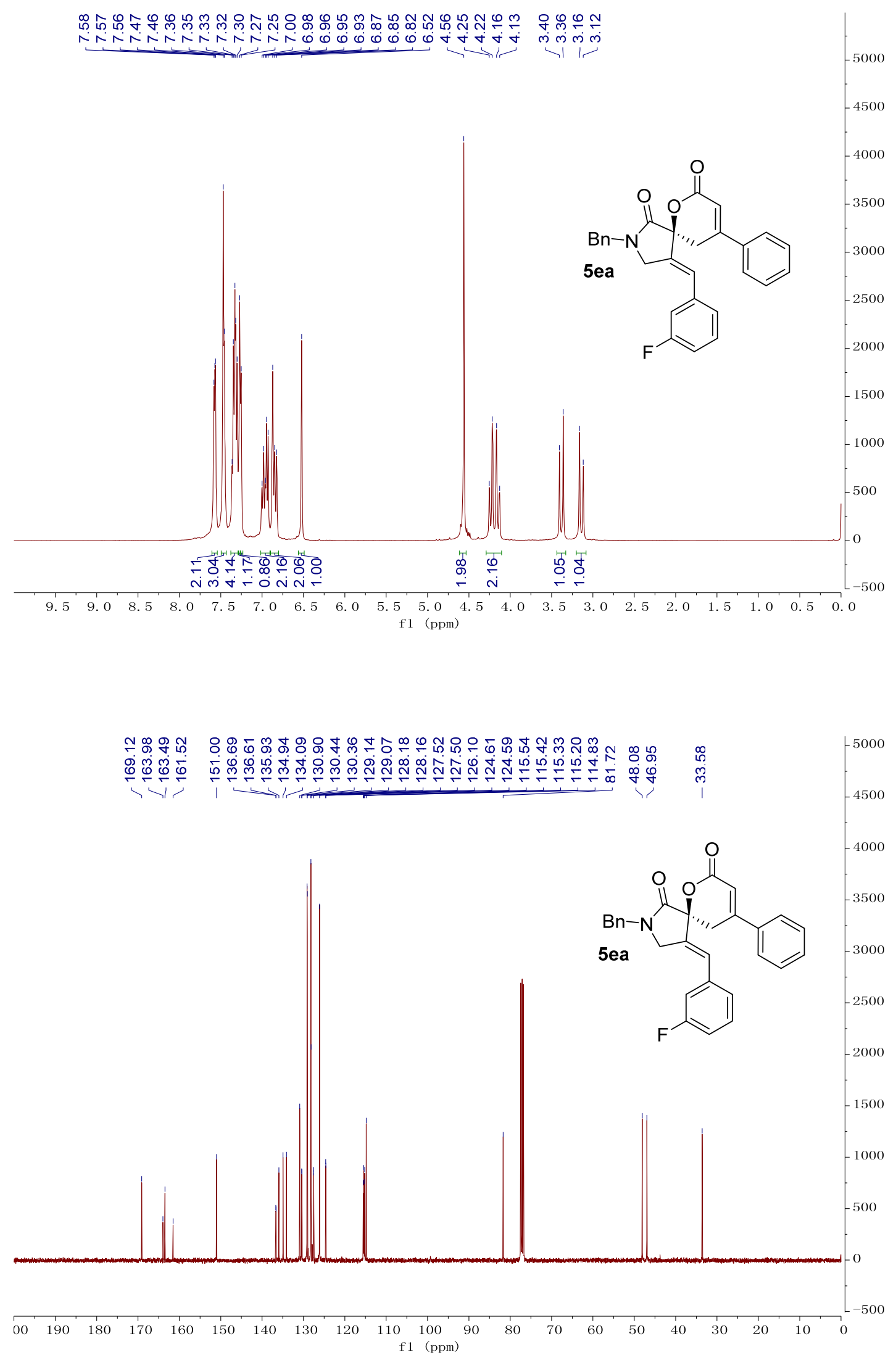

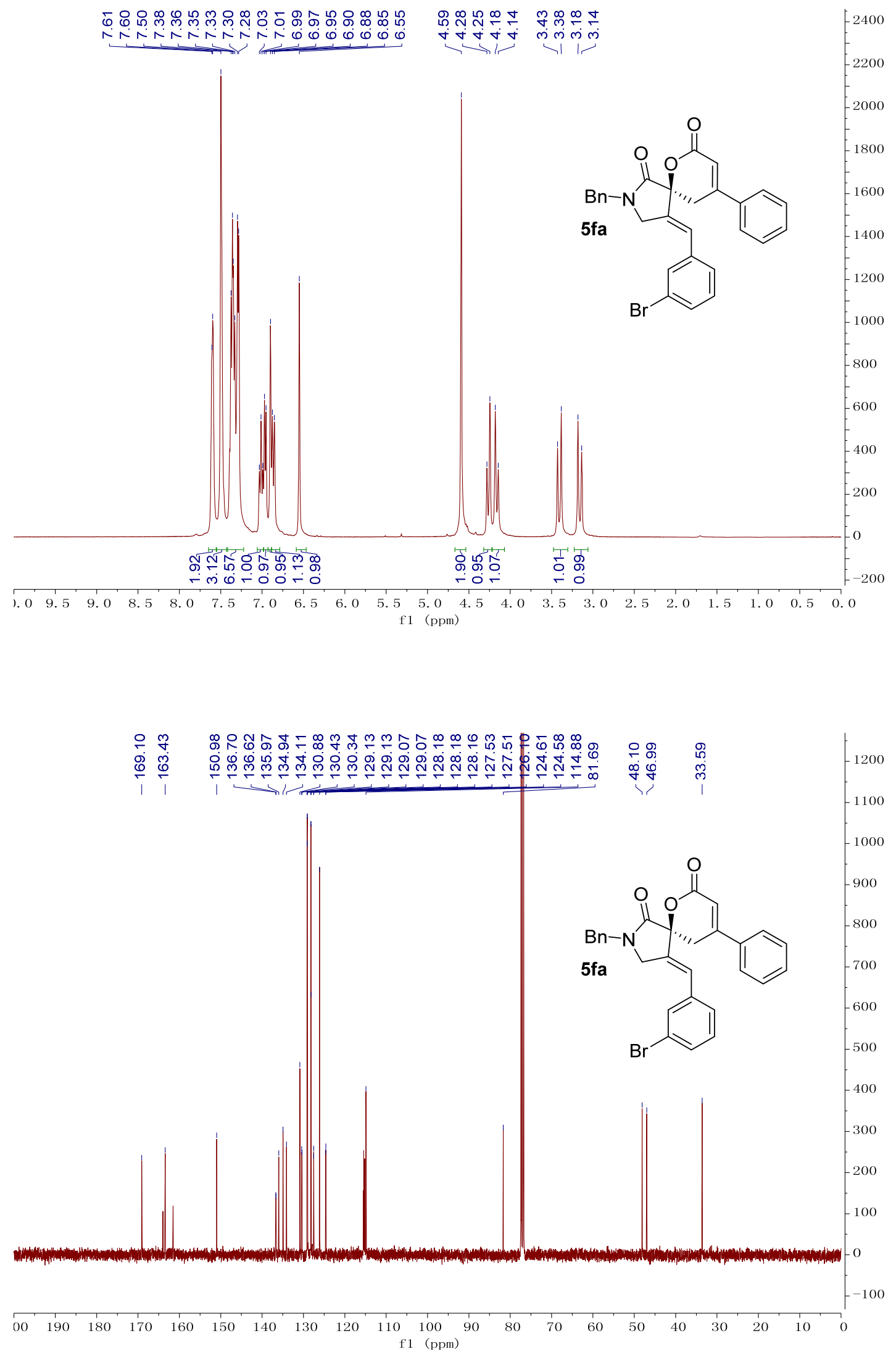


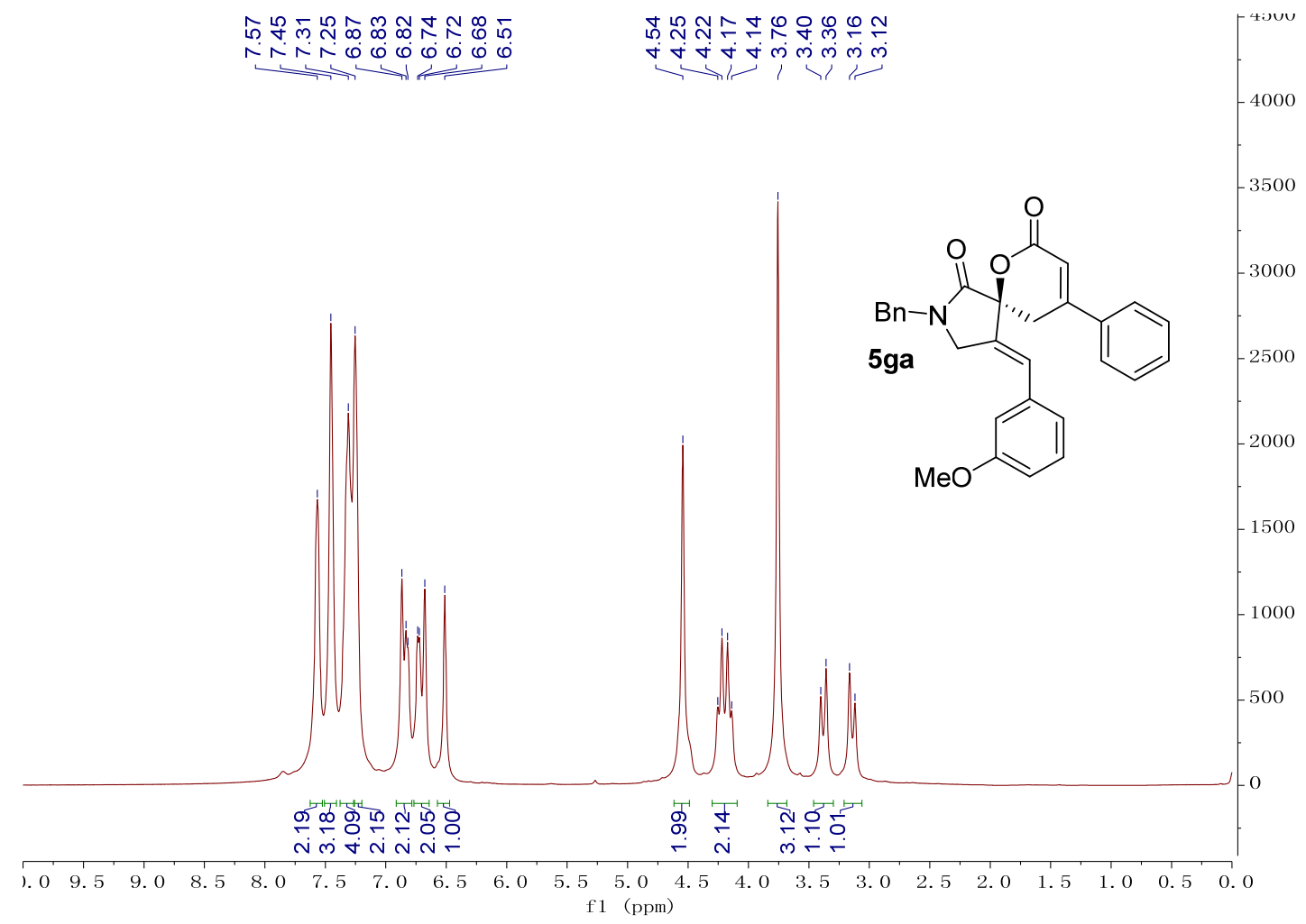

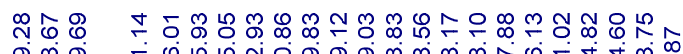

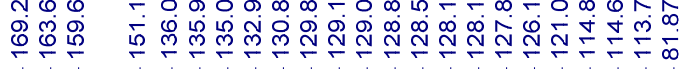

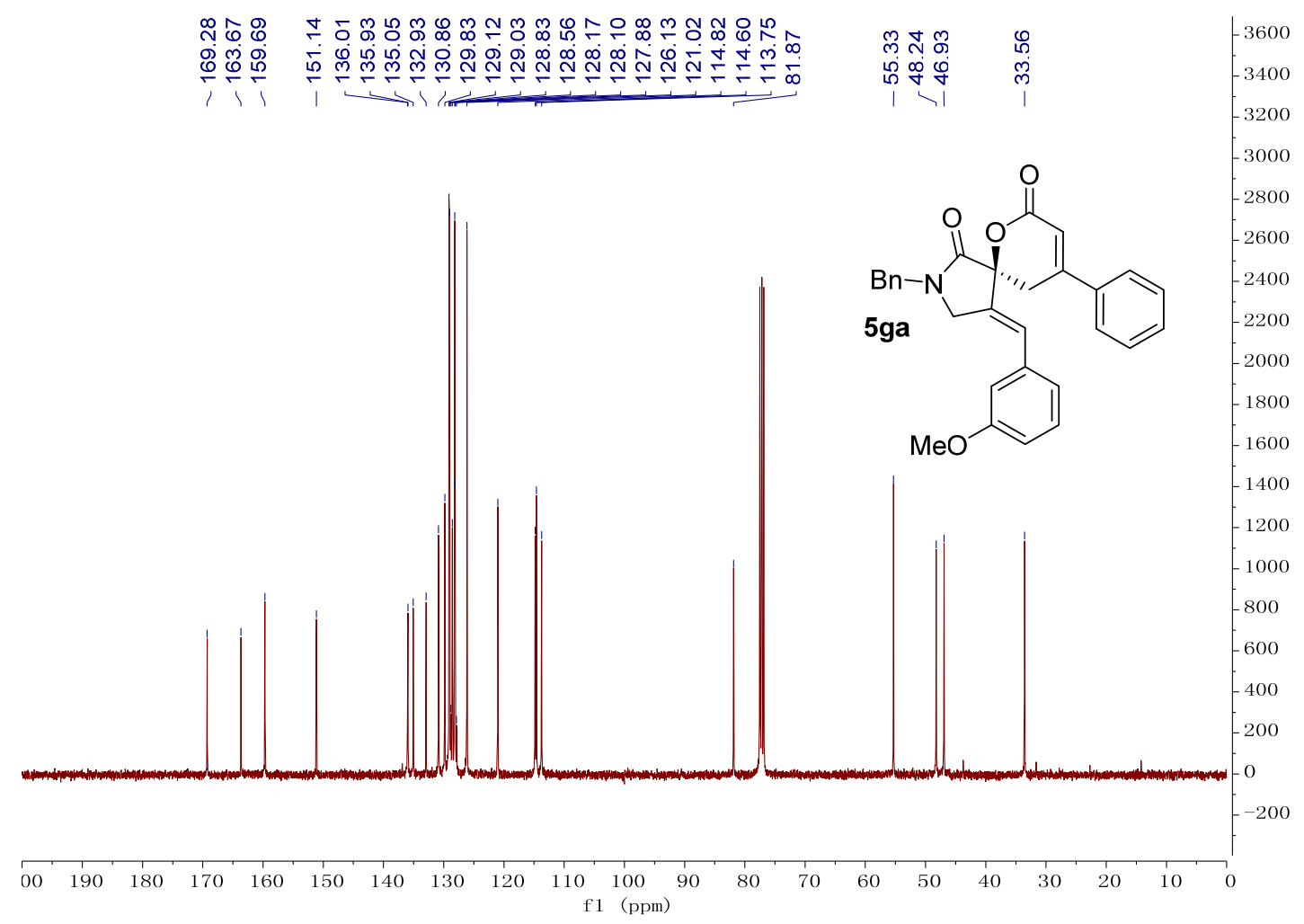



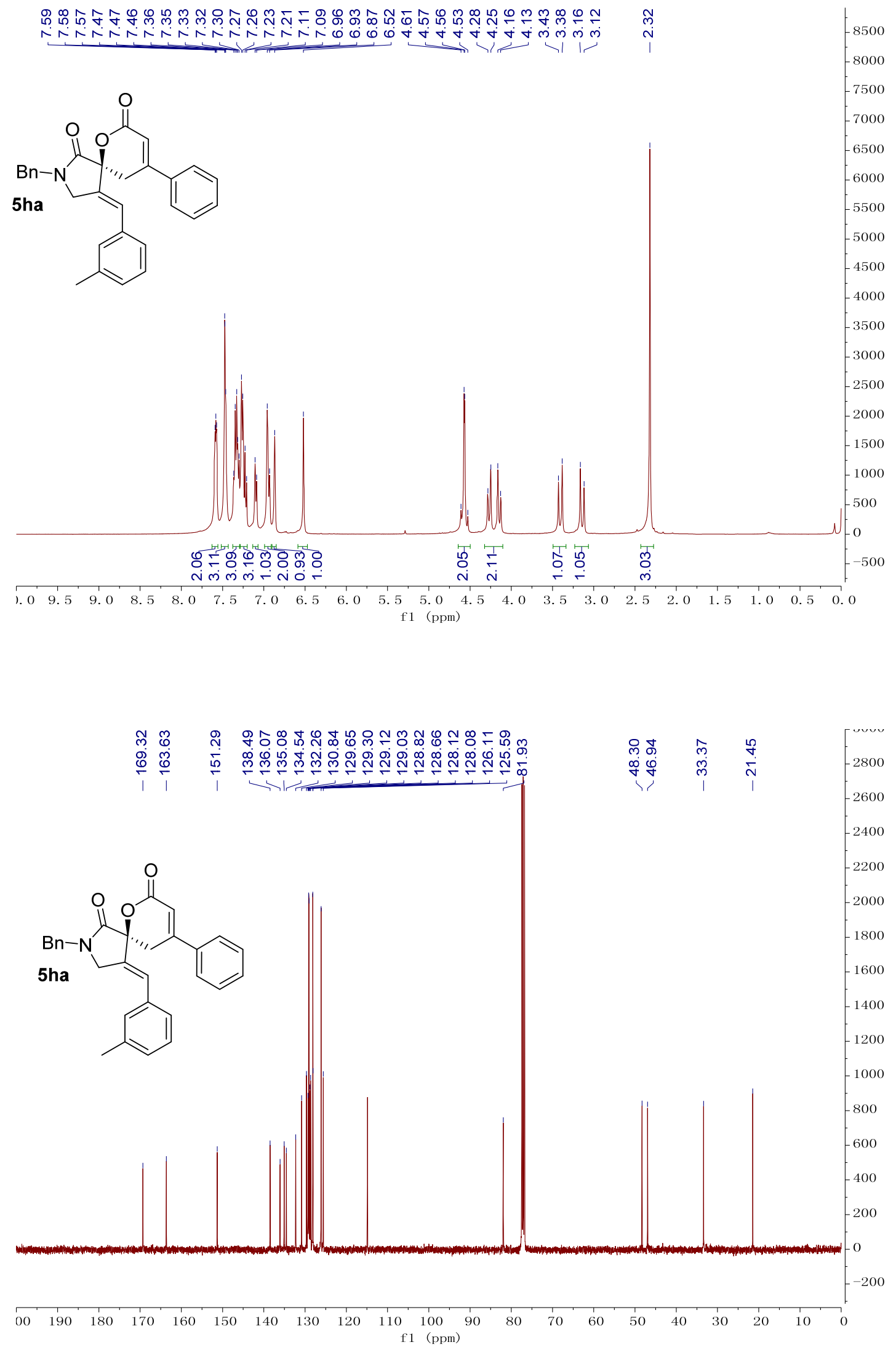


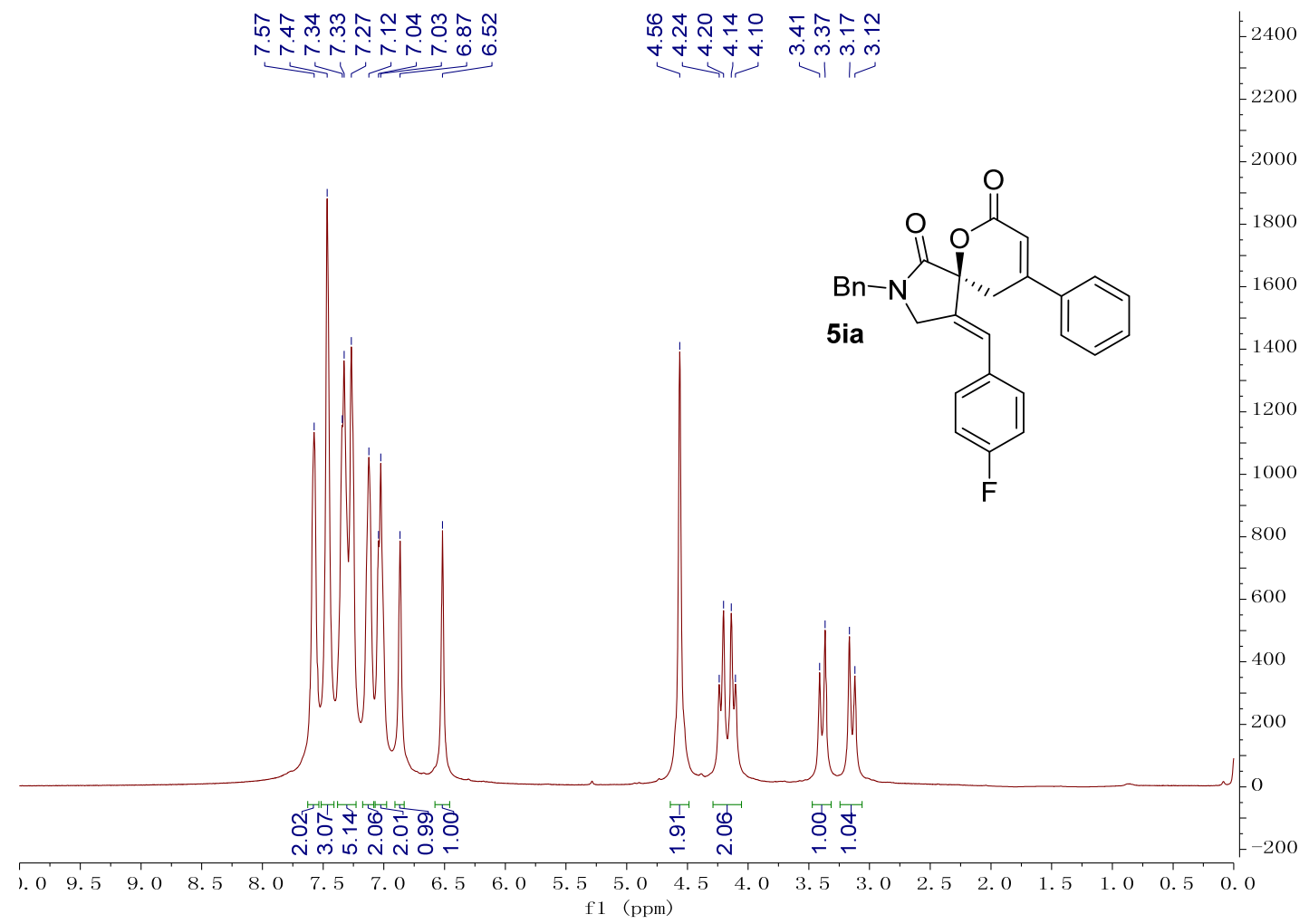

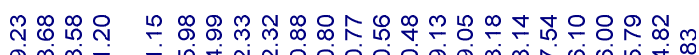

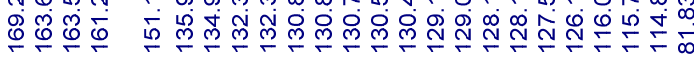

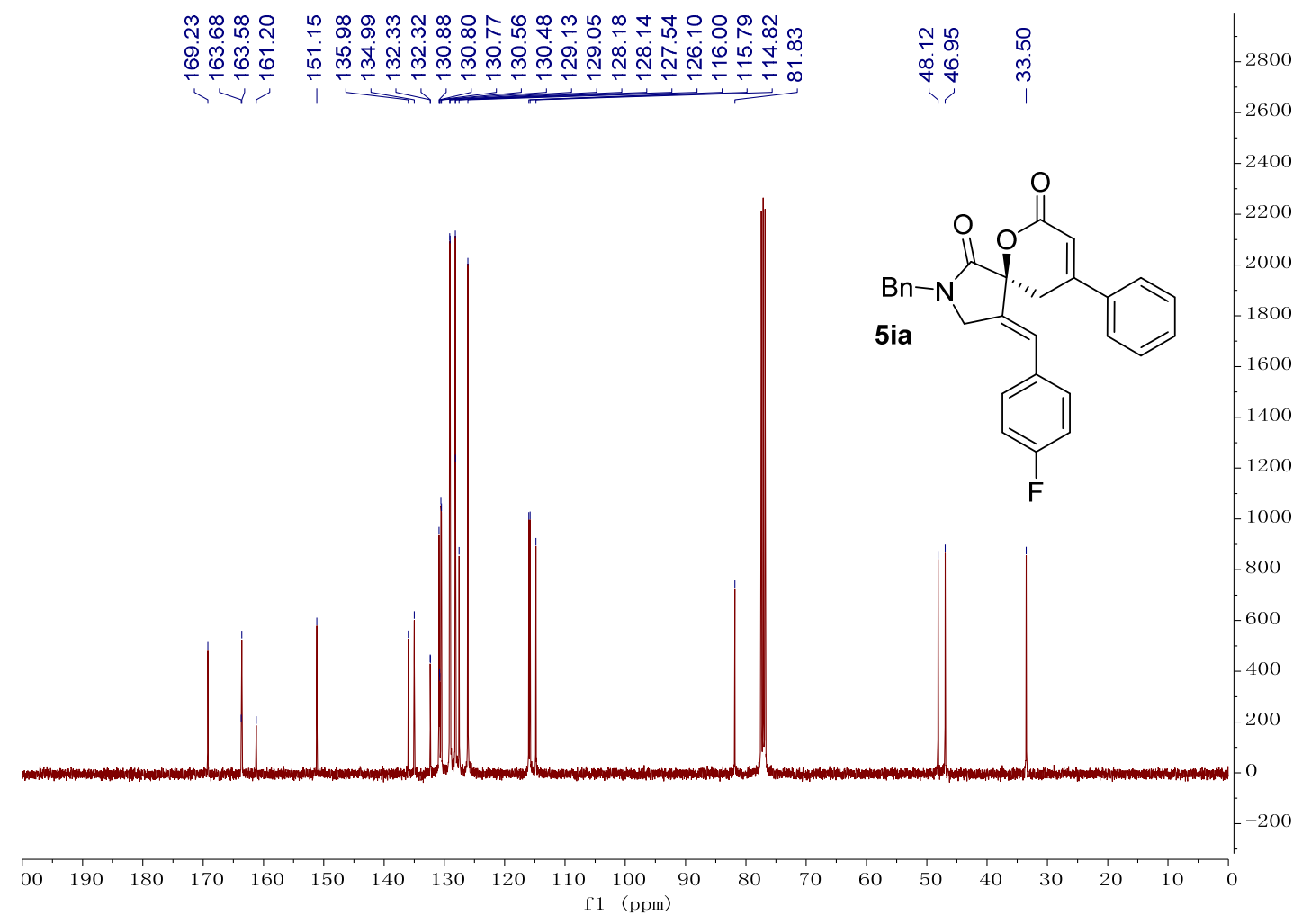



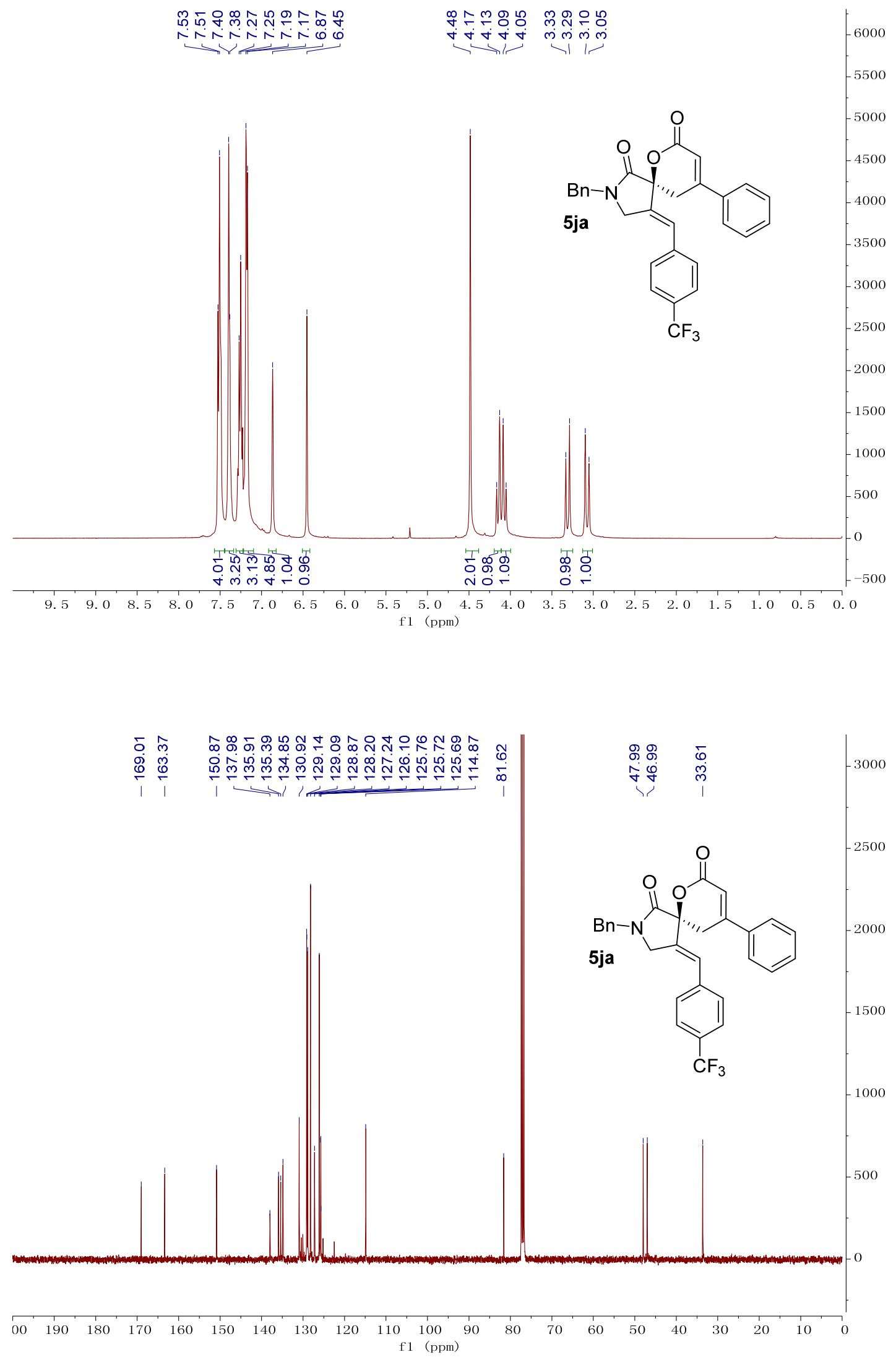

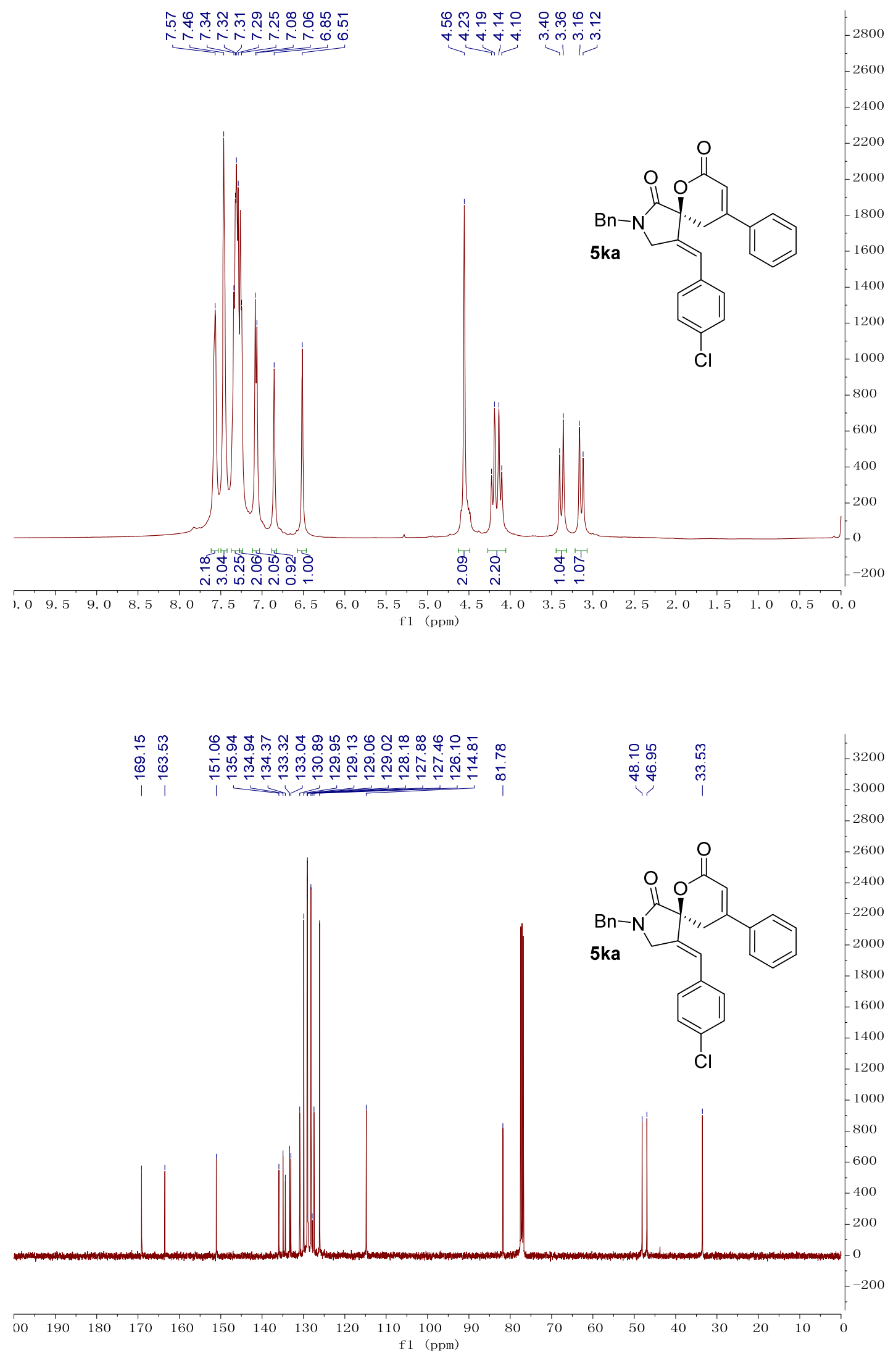

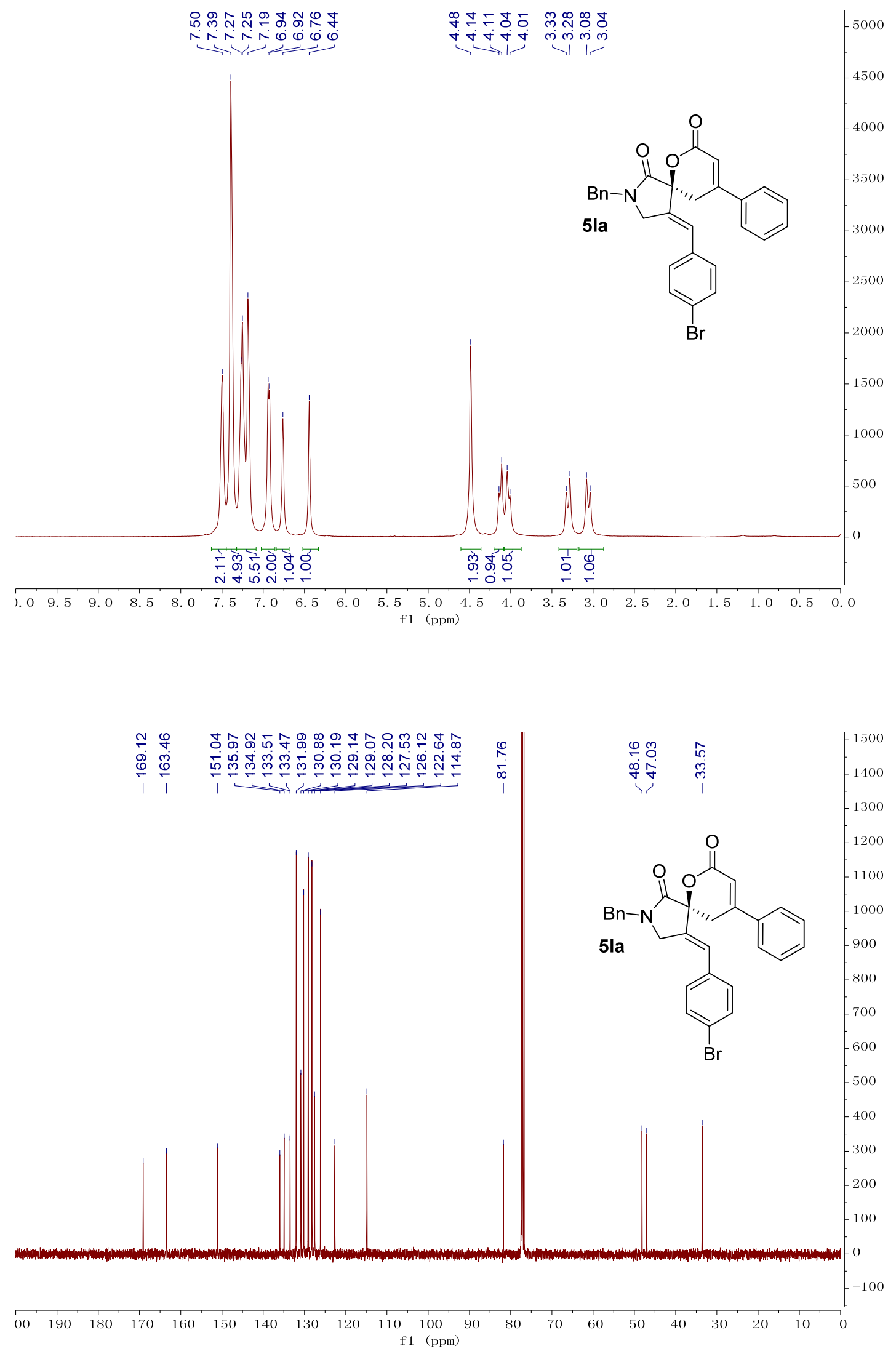

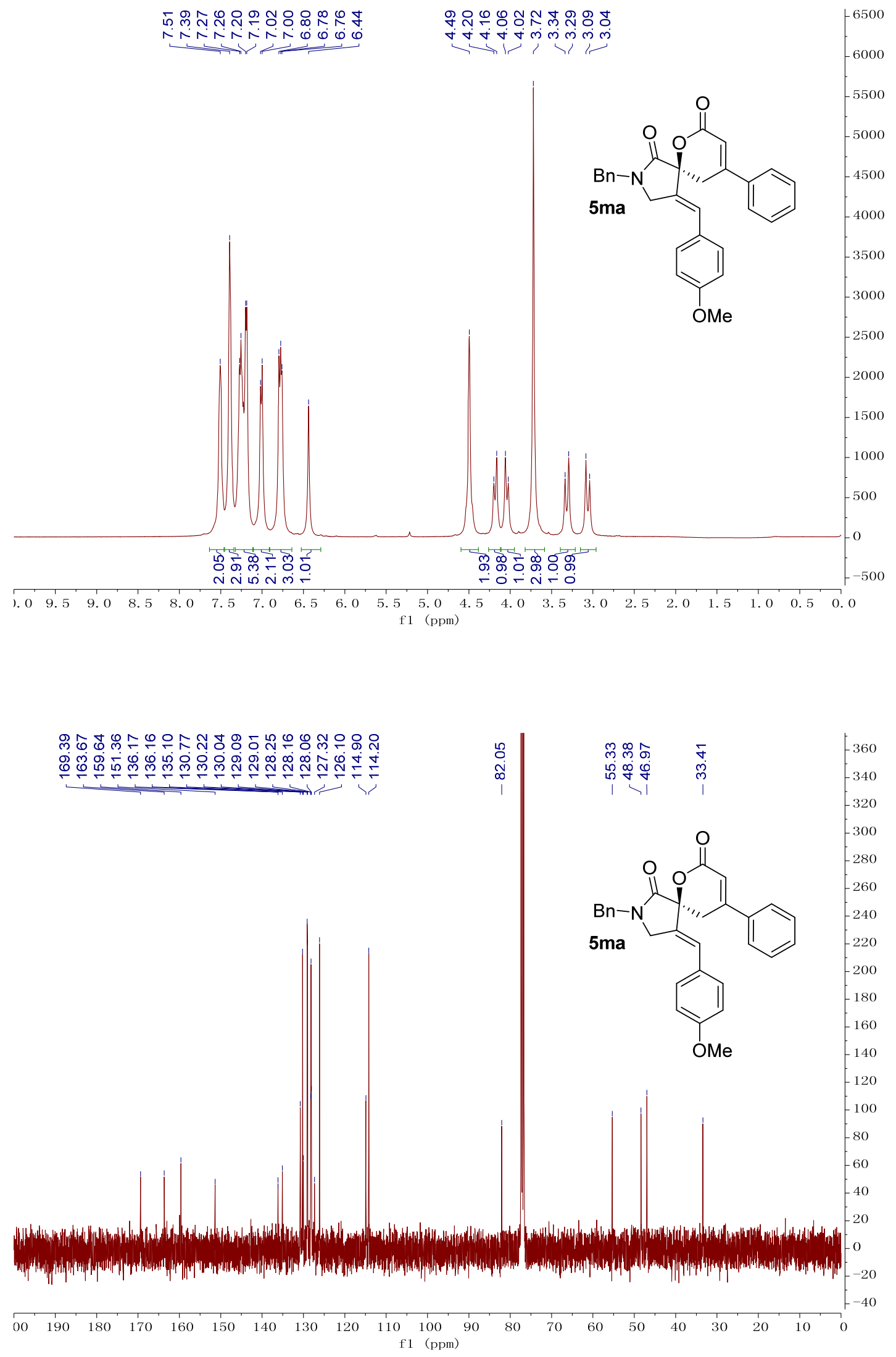

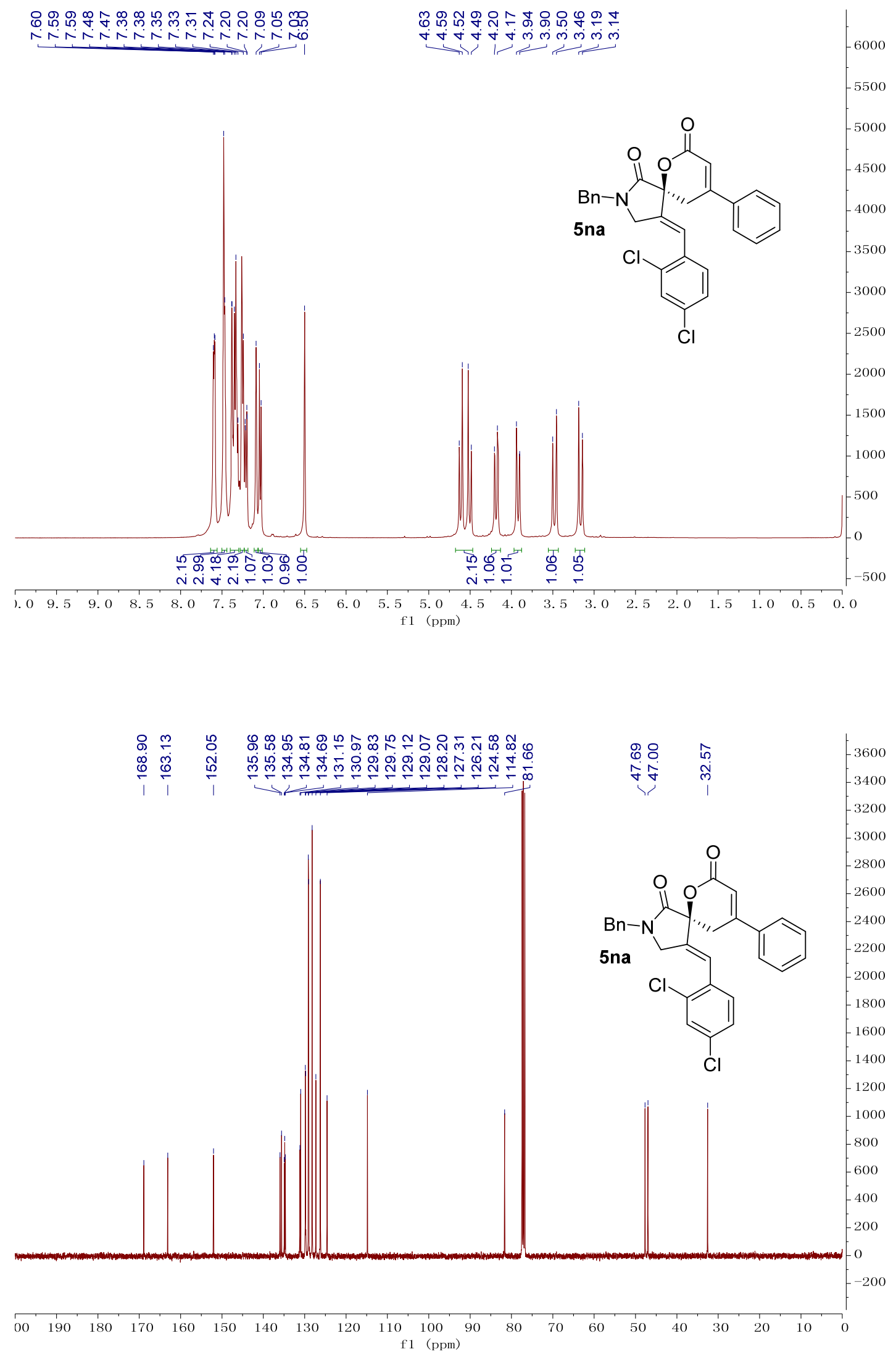

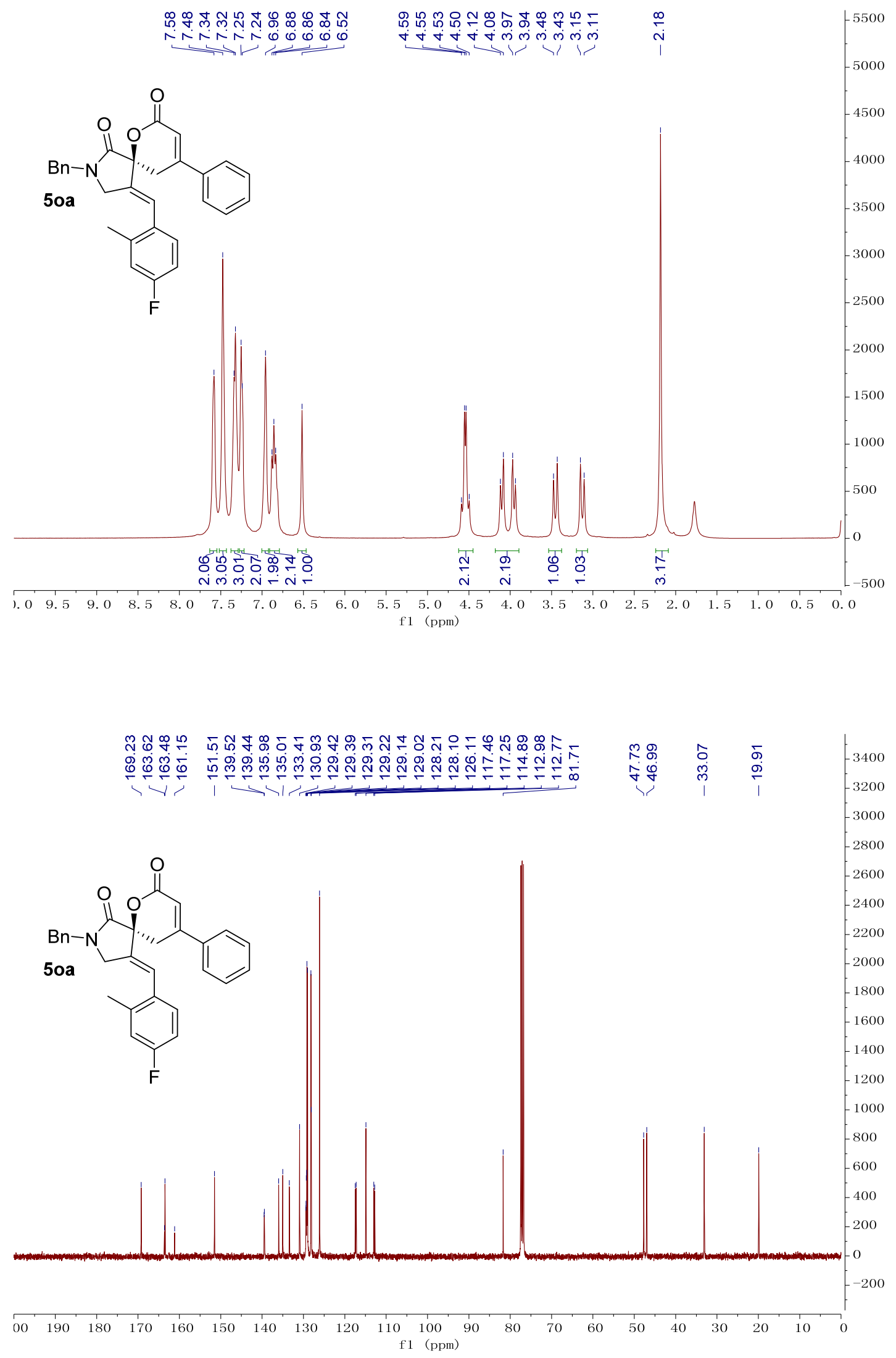

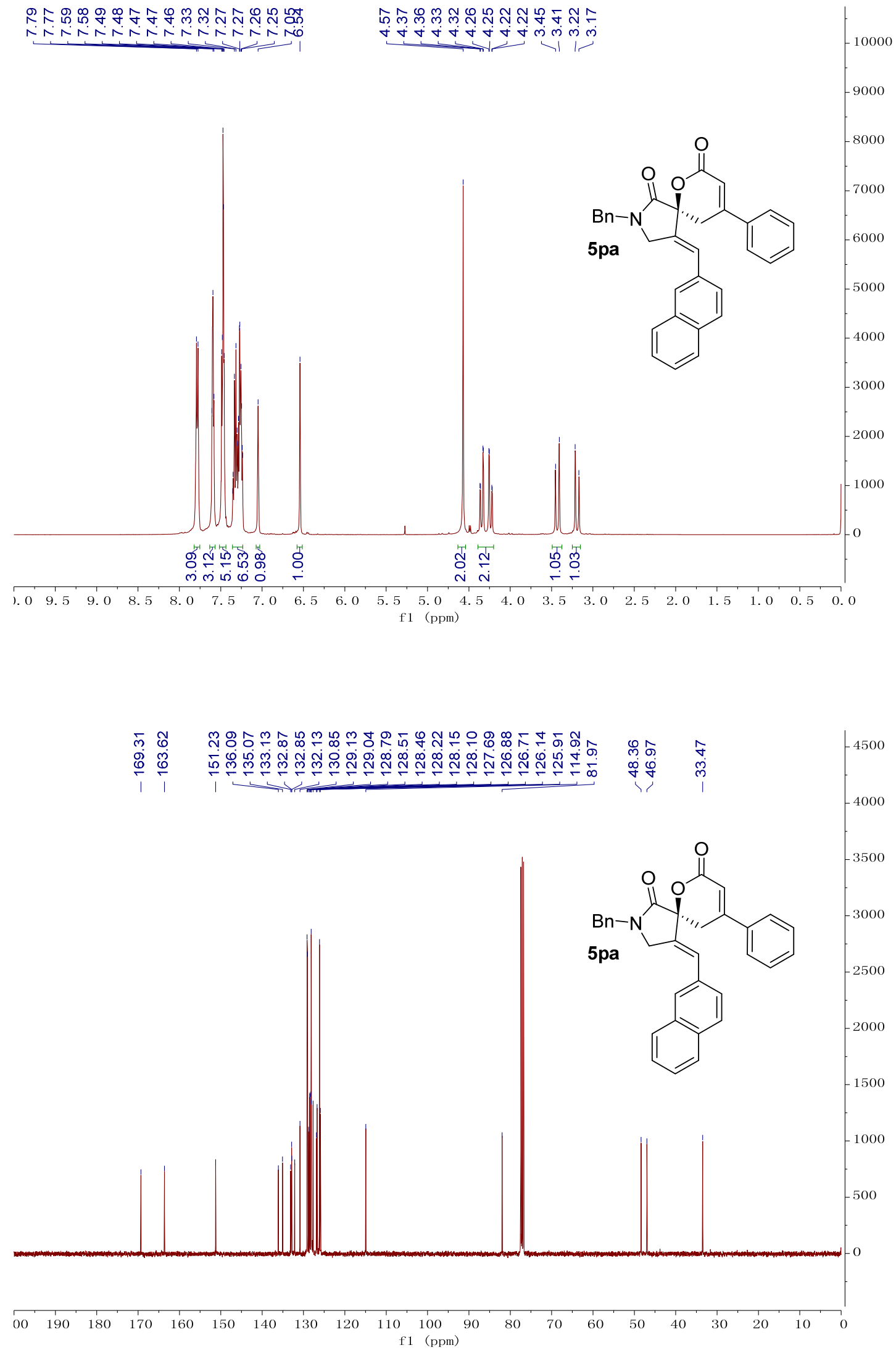


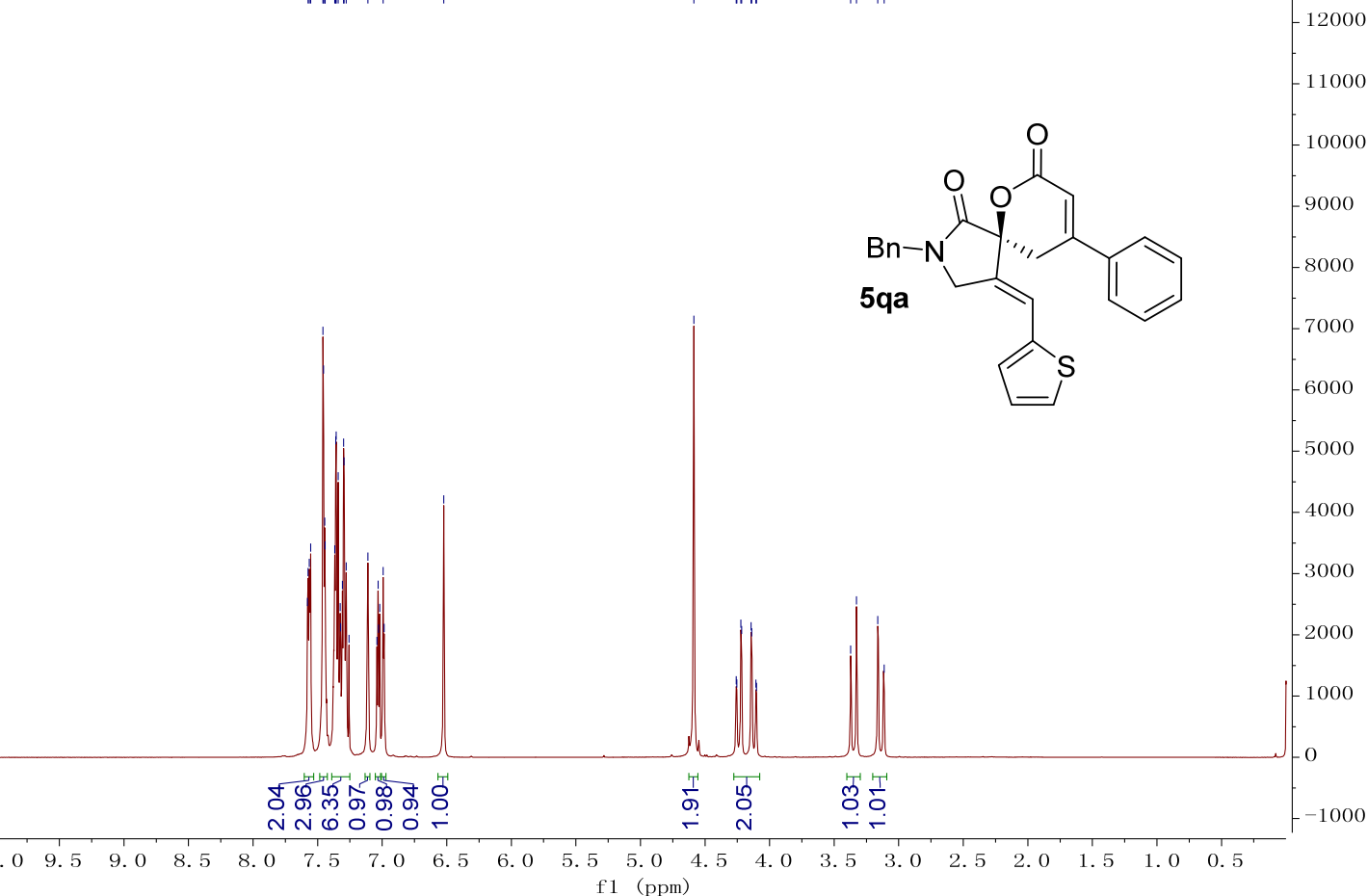

(난

i

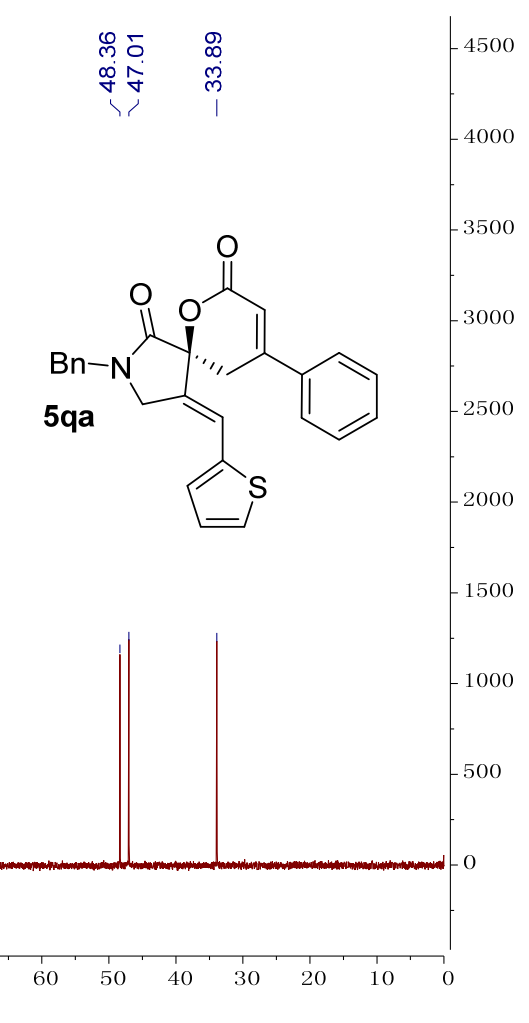

$\begin{array}{lllllllllllllllllllll}00 & 190 & 180 & 170 & 160 & 150 & 140 & 130 & 120 & 110 & 100 & 90 & 80 & 70 & 60 & 50 & 40 & 30 & 20 & 10 & 0\end{array}$ 


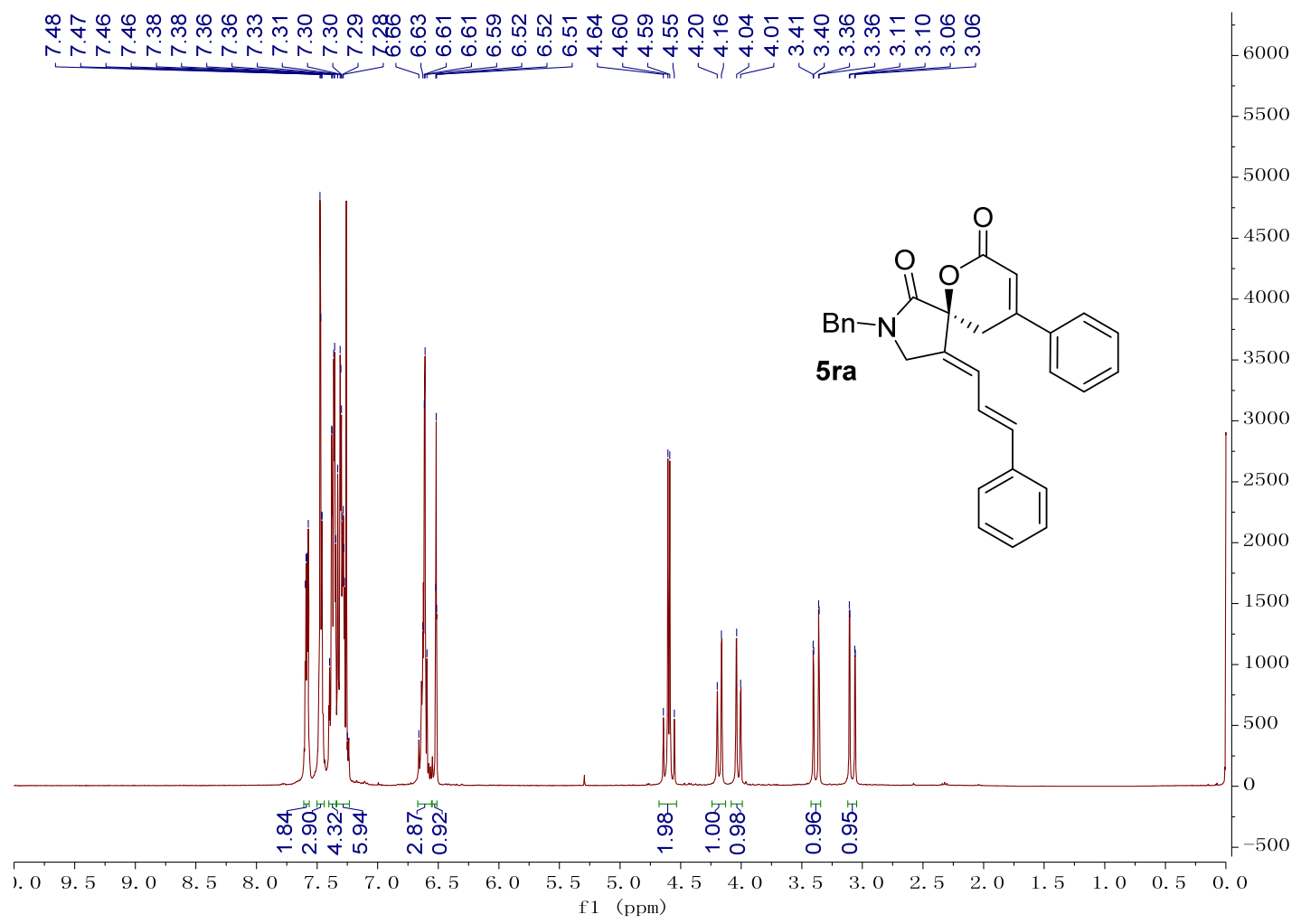

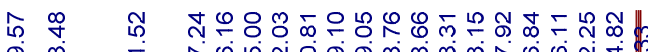

○.

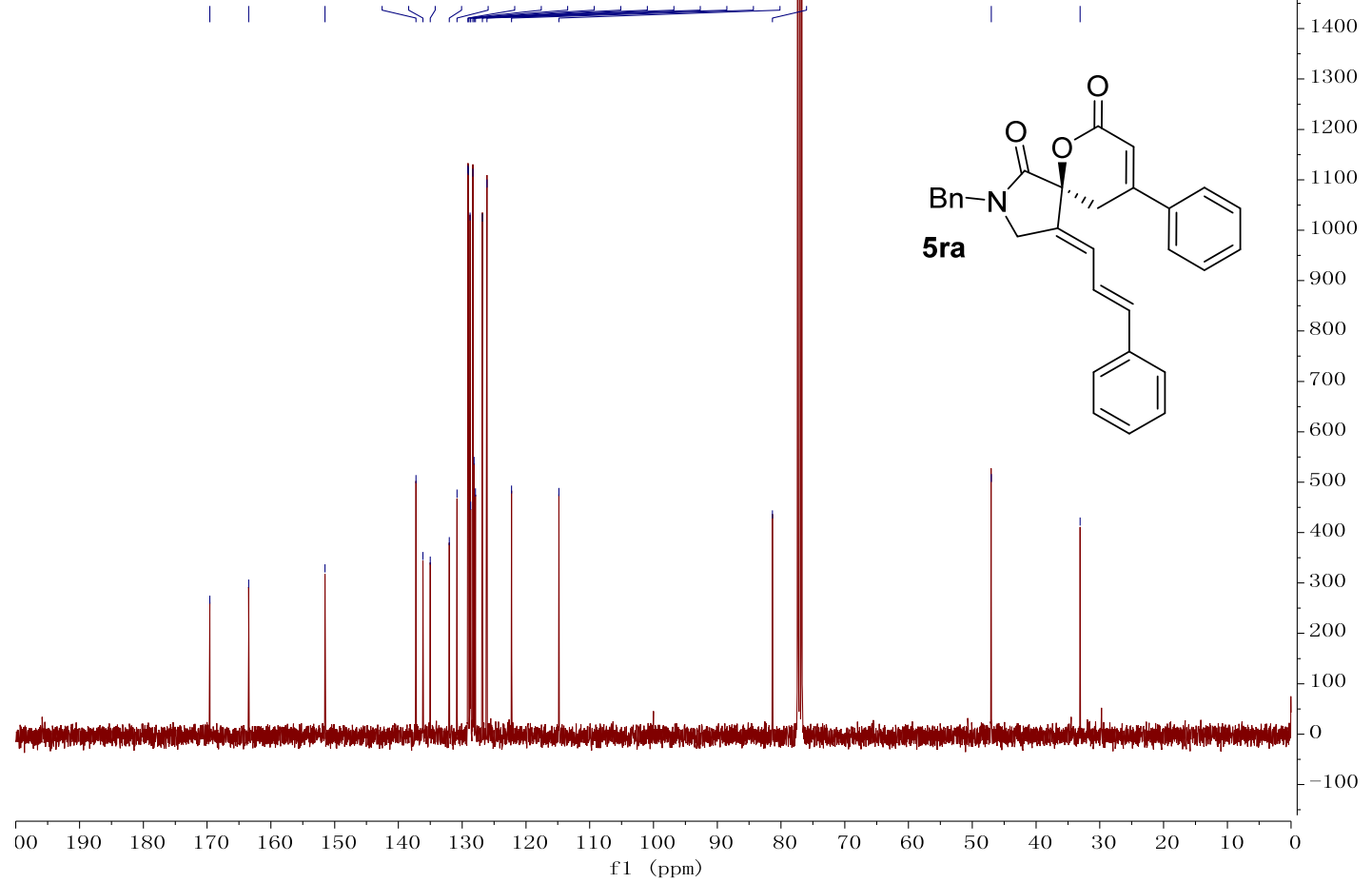



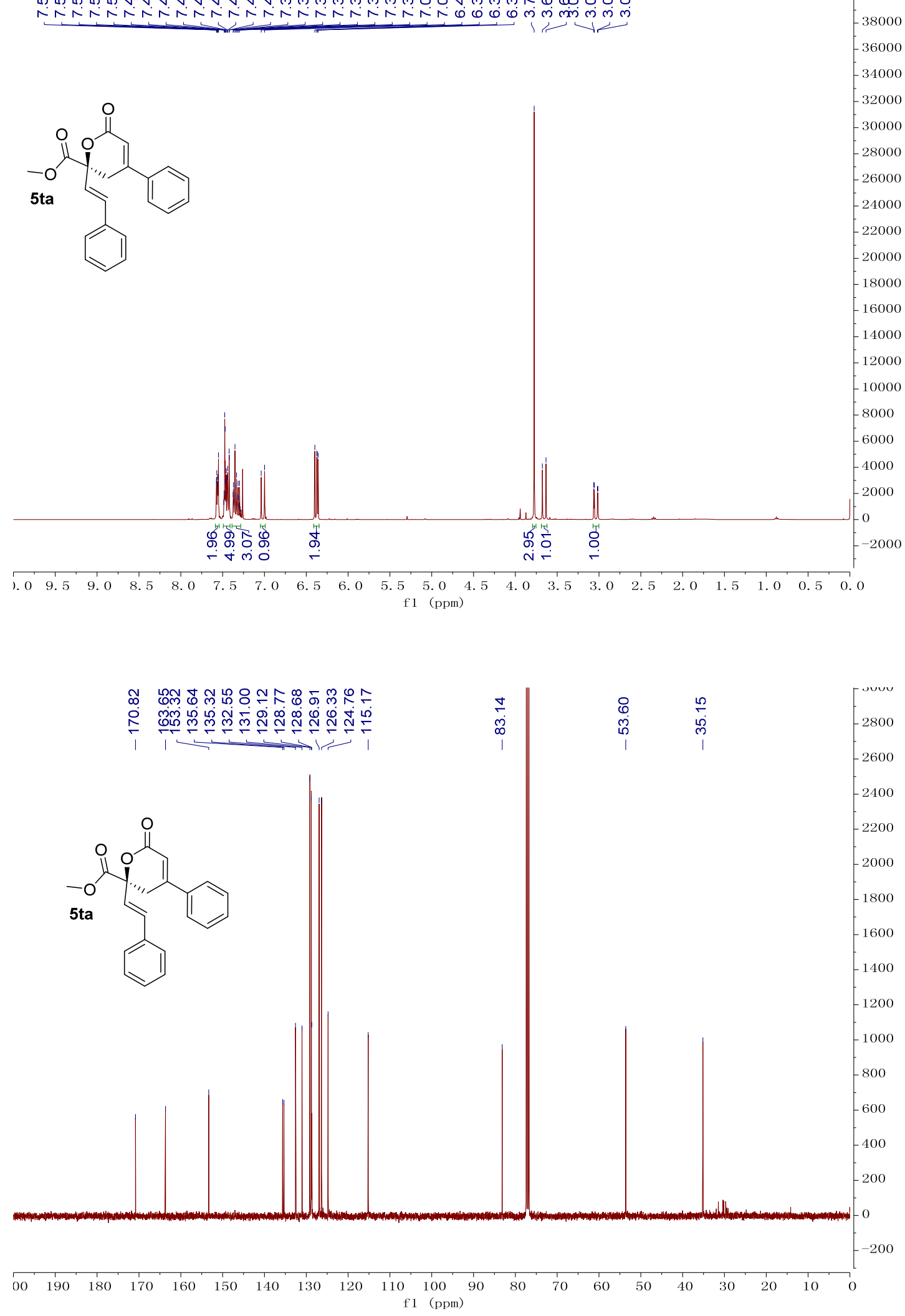

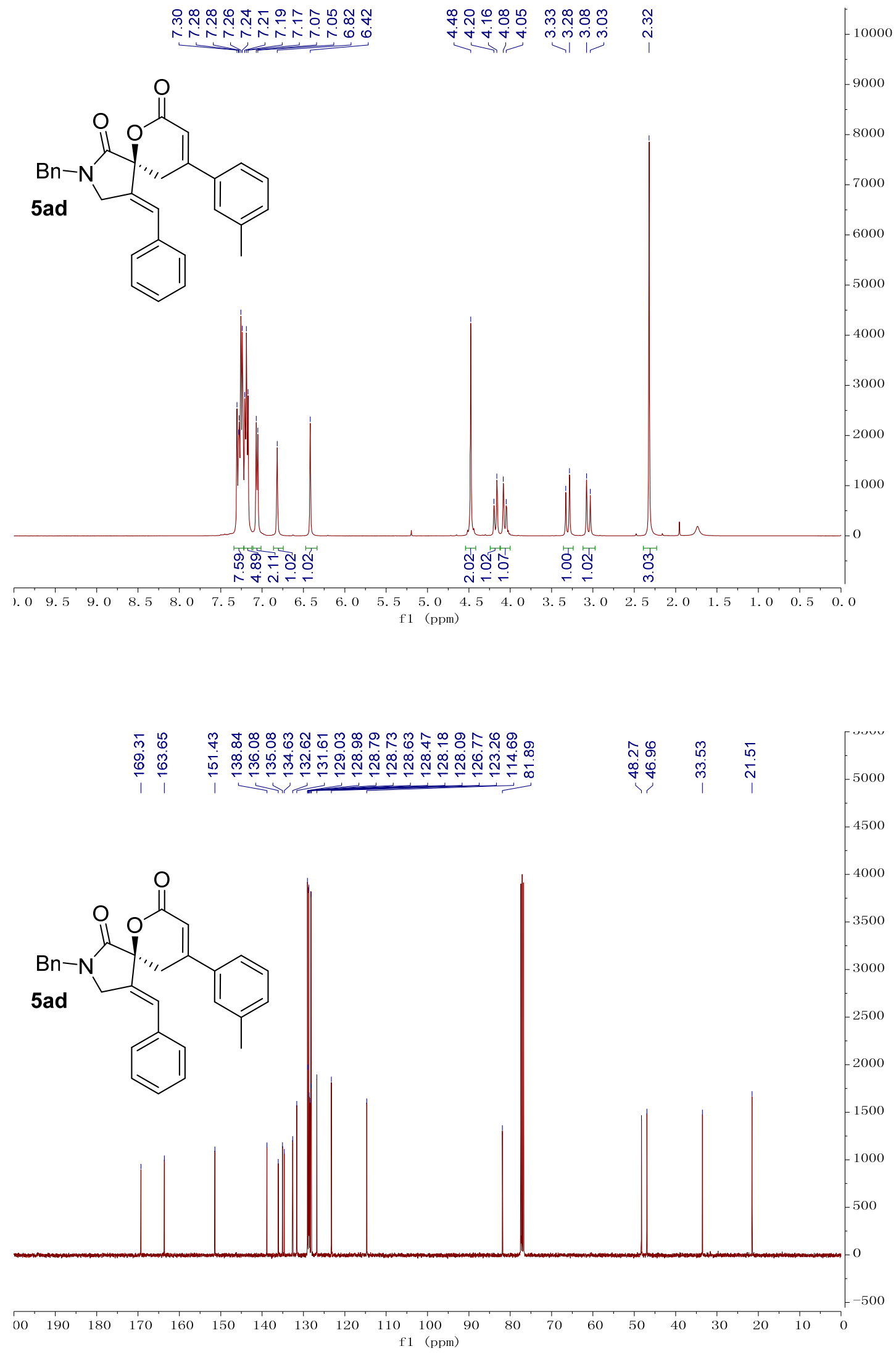

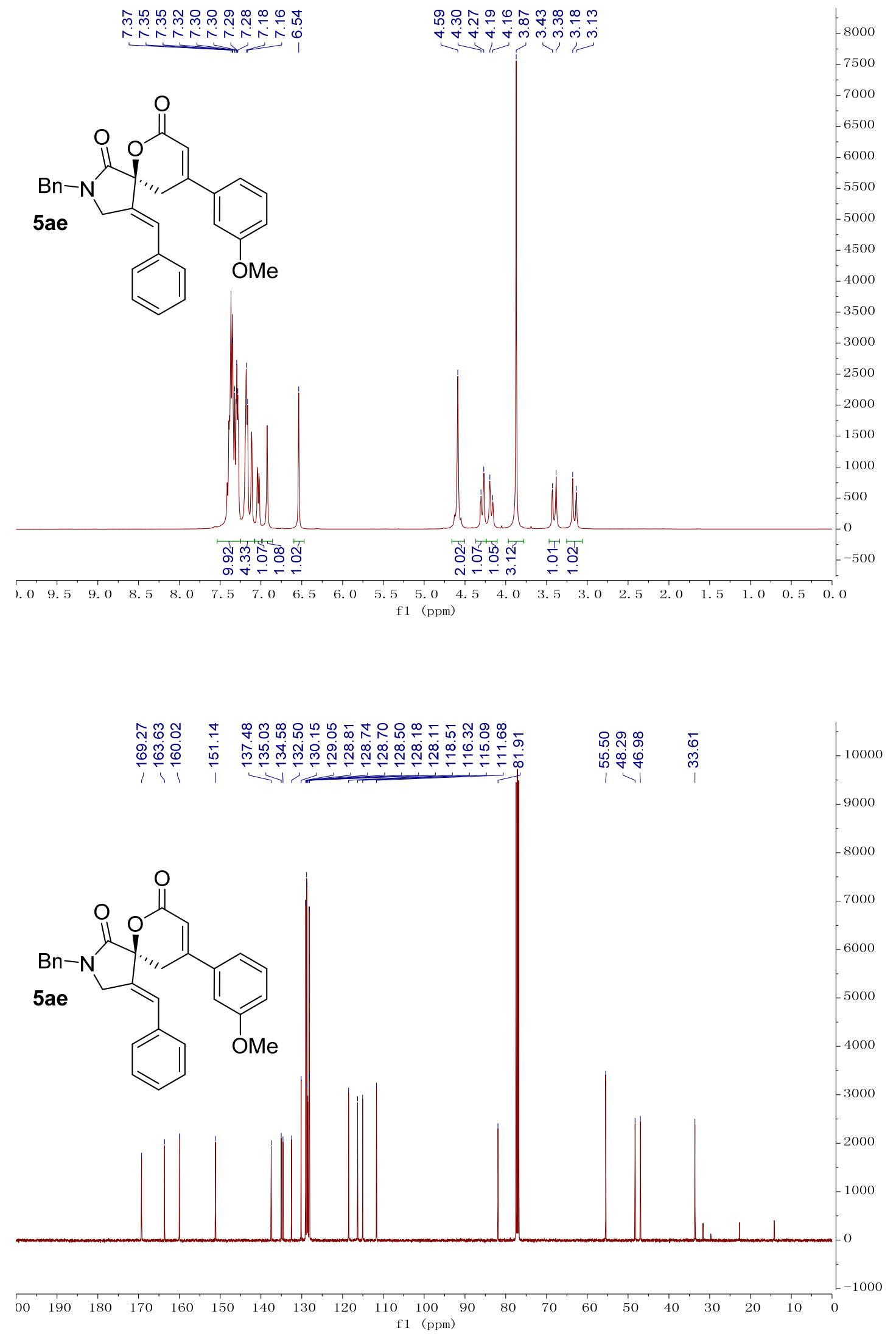

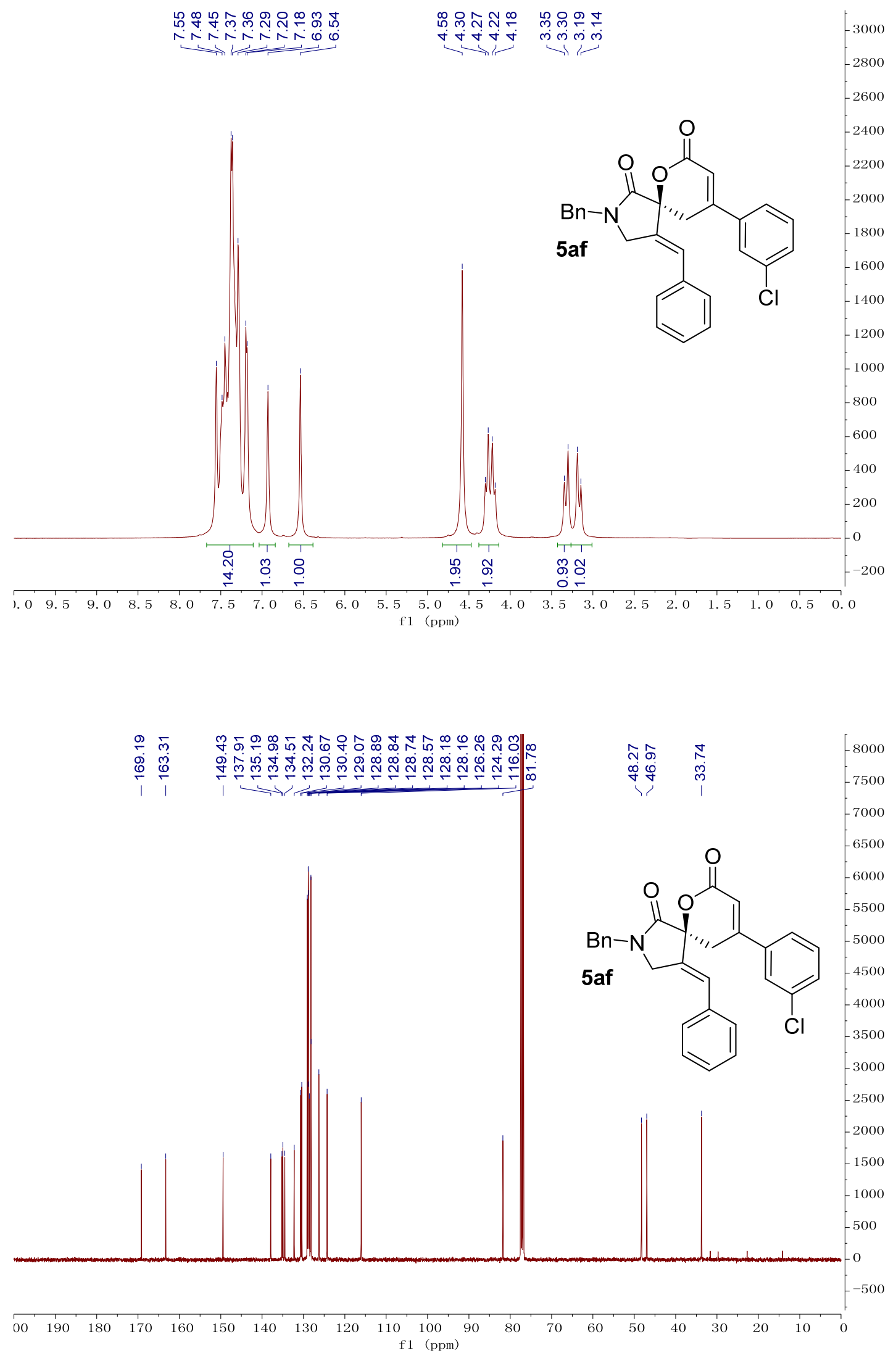

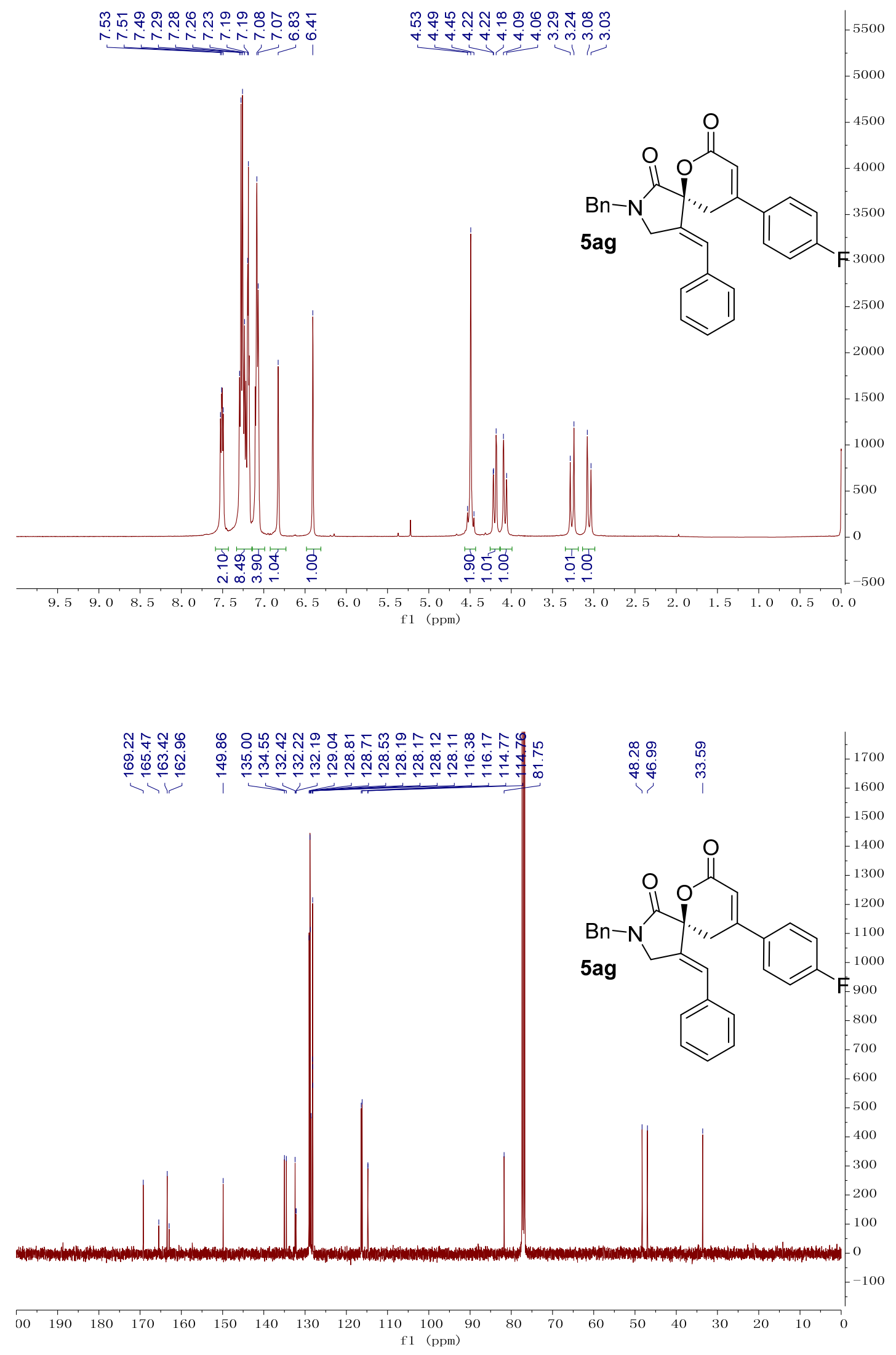

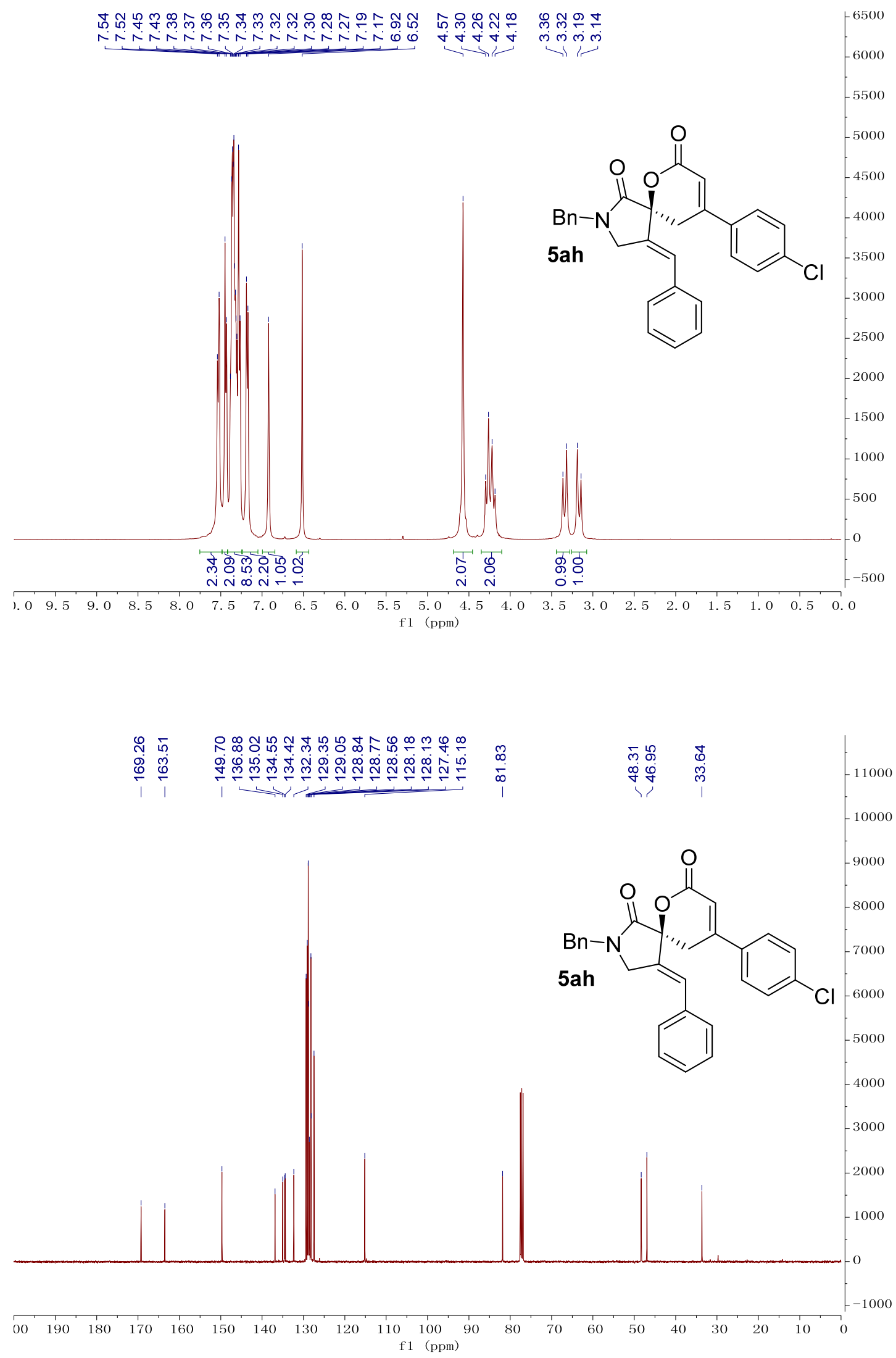

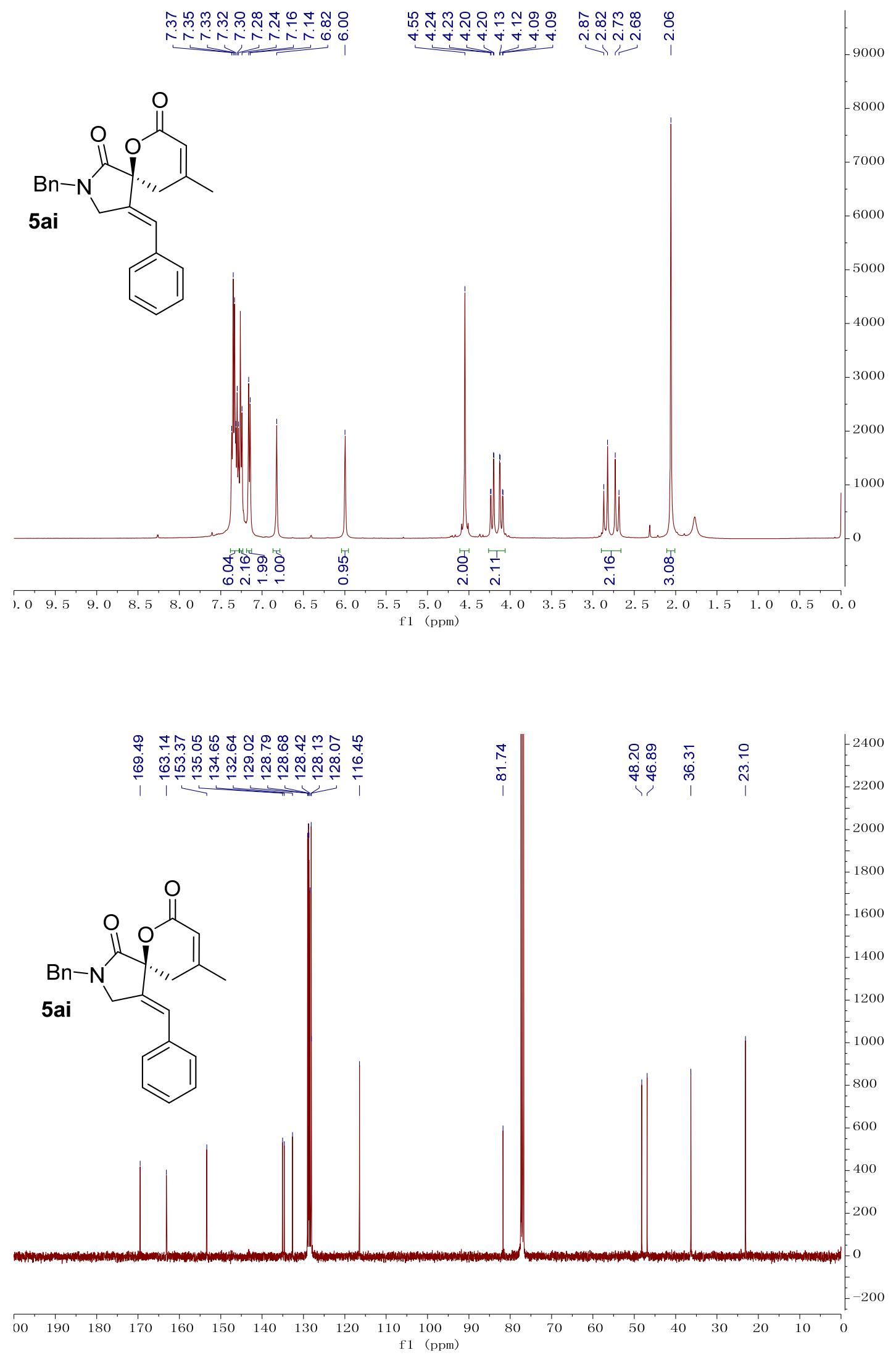

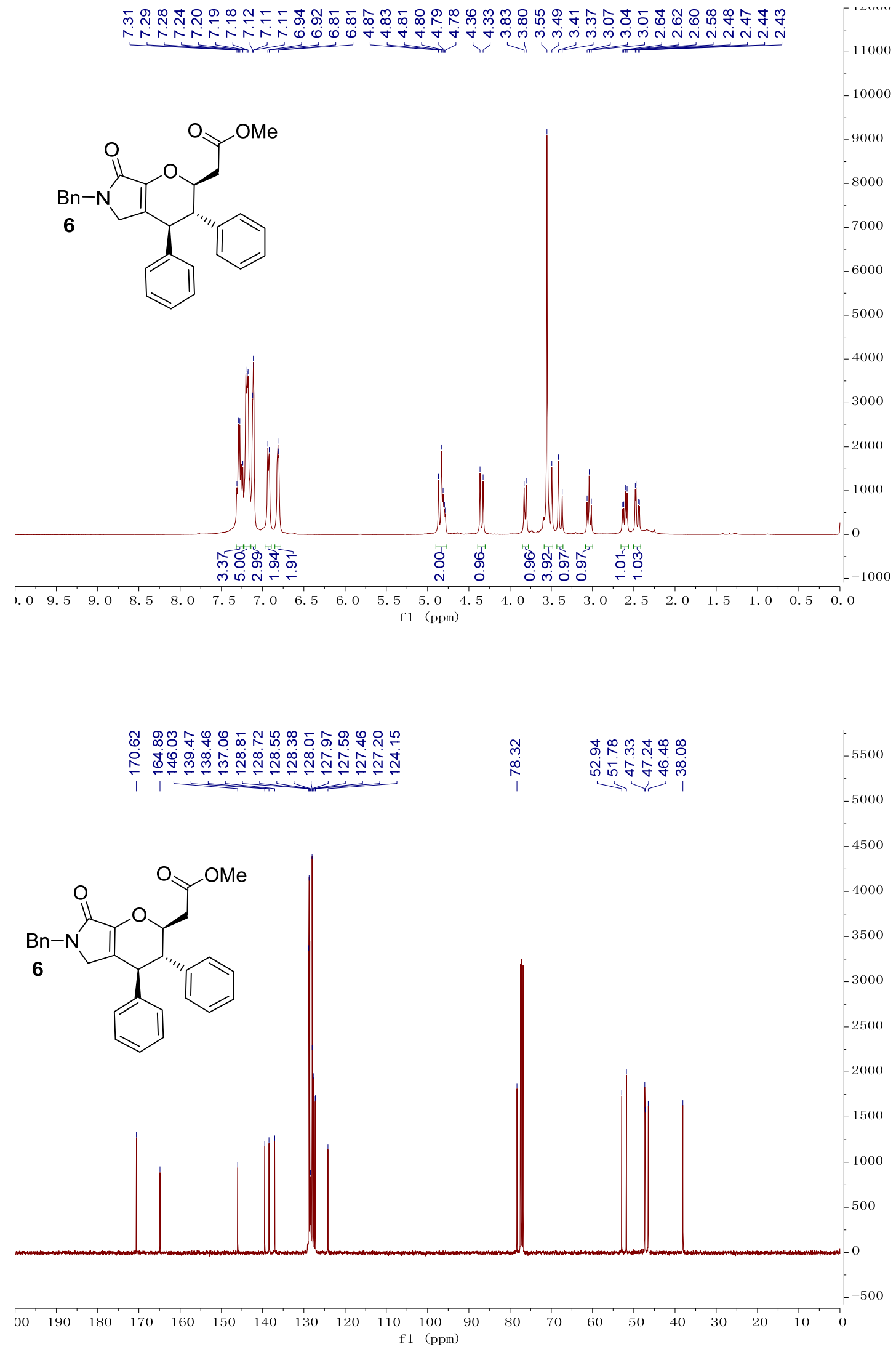

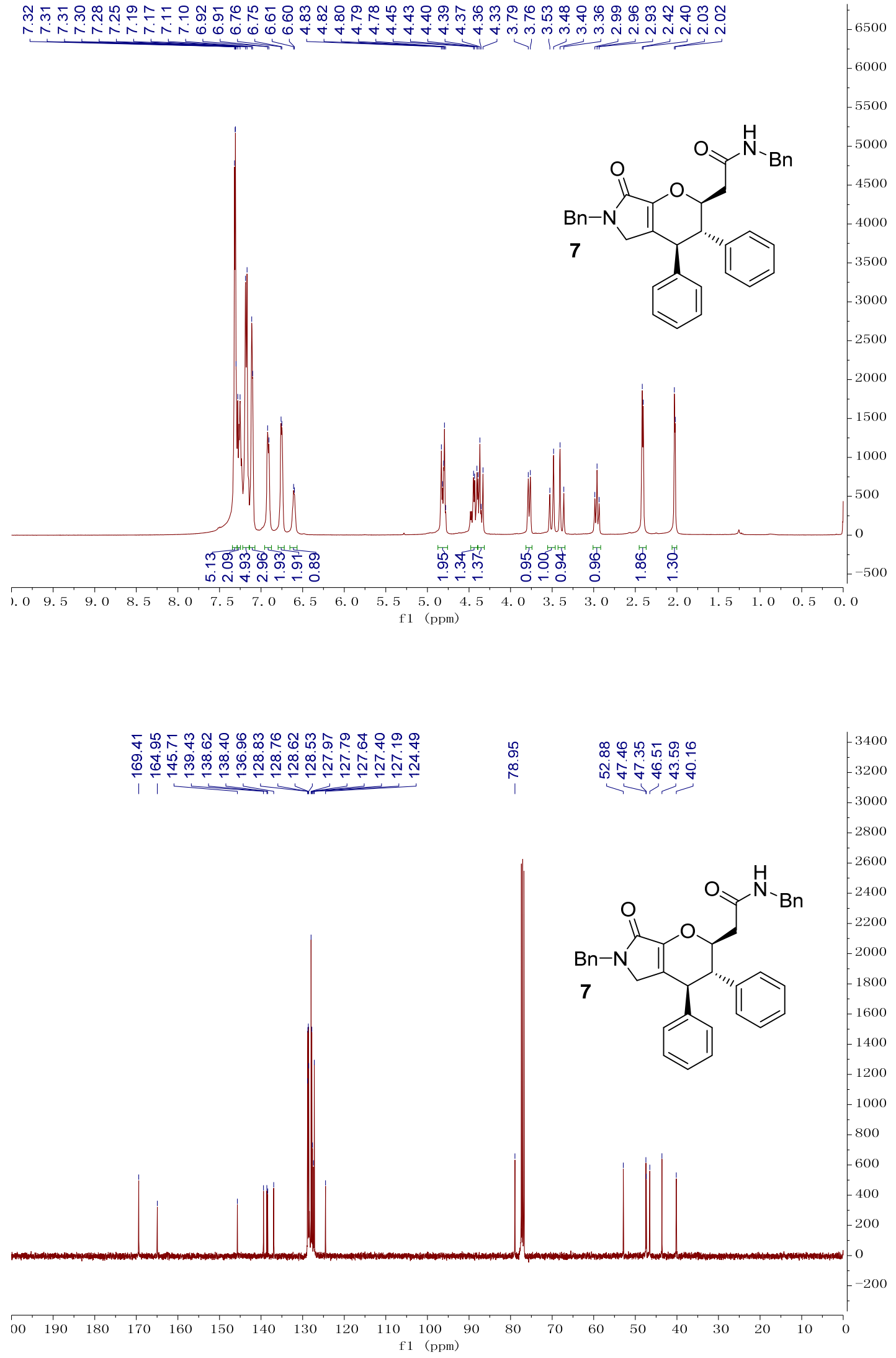

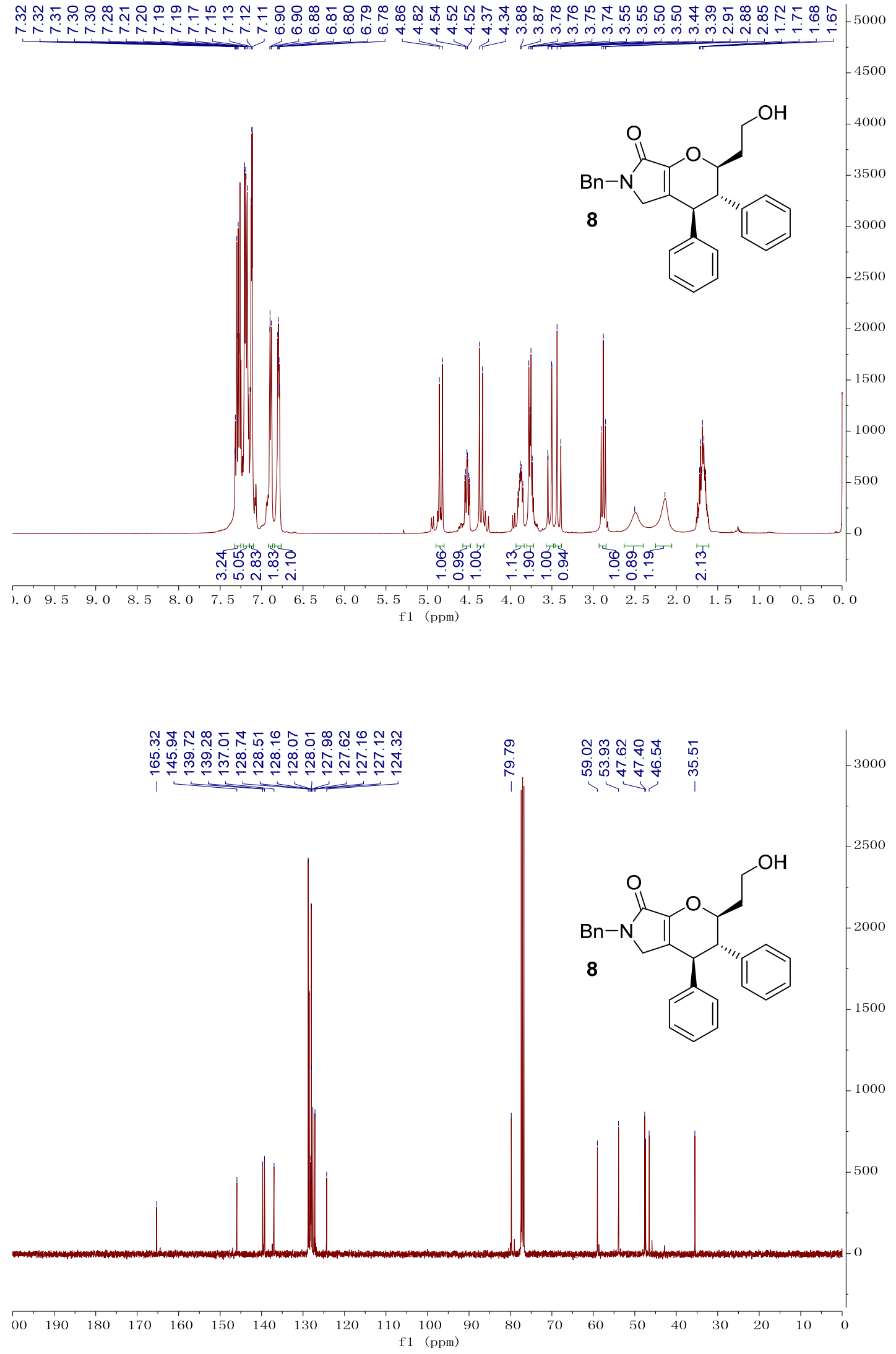

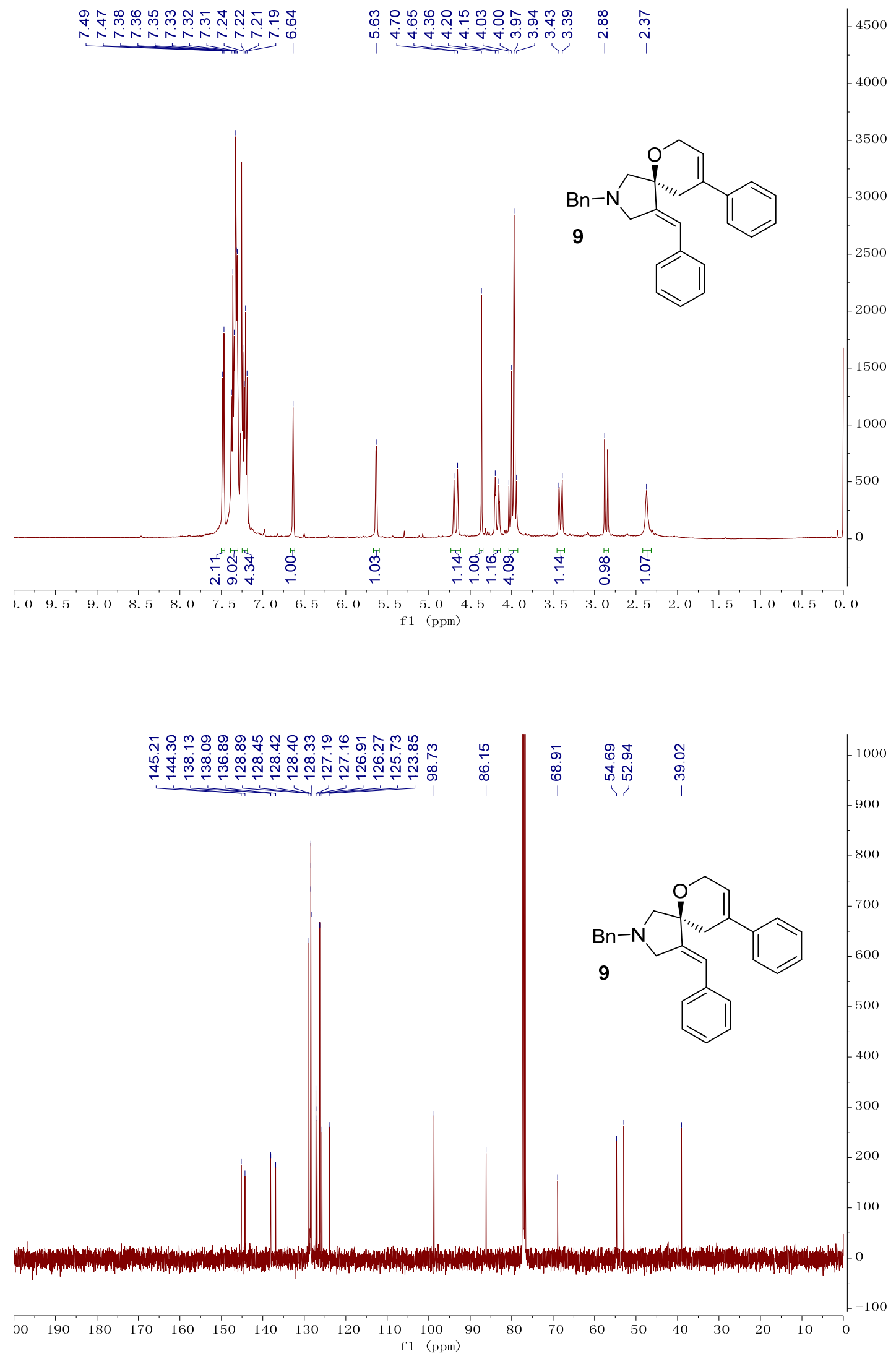


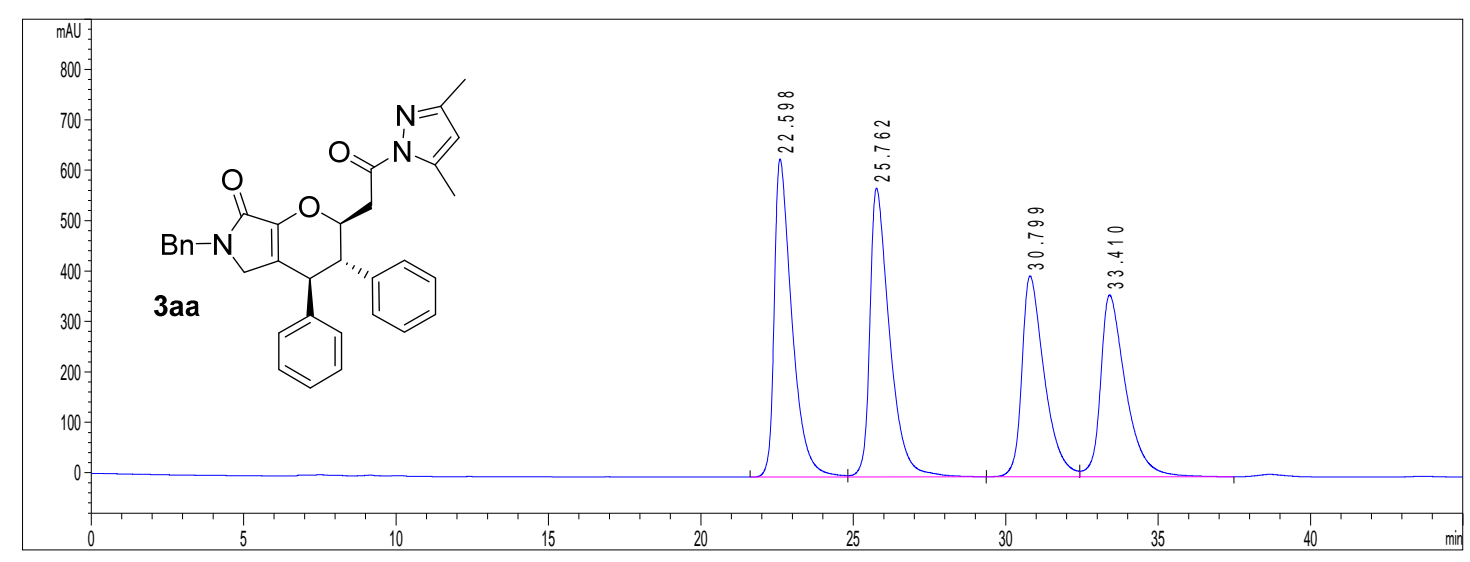

\begin{tabular}{|l|l|l|l|}
\hline & Retention & Area & Area\% \\
\hline 1 & 22.598 & $2.54794 \mathrm{e} 4$ & 27.5707 \\
\hline 2 & 25.762 & $2.57559 \mathrm{e} 4$ & 27.8700 \\
\hline 3 & 30.799 & $2.04310 \mathrm{e} 4$ & 22.1080 \\
\hline 4 & 33.410 & $2.07483 \mathrm{e} 4$ & 22.4513 \\
\hline
\end{tabular}

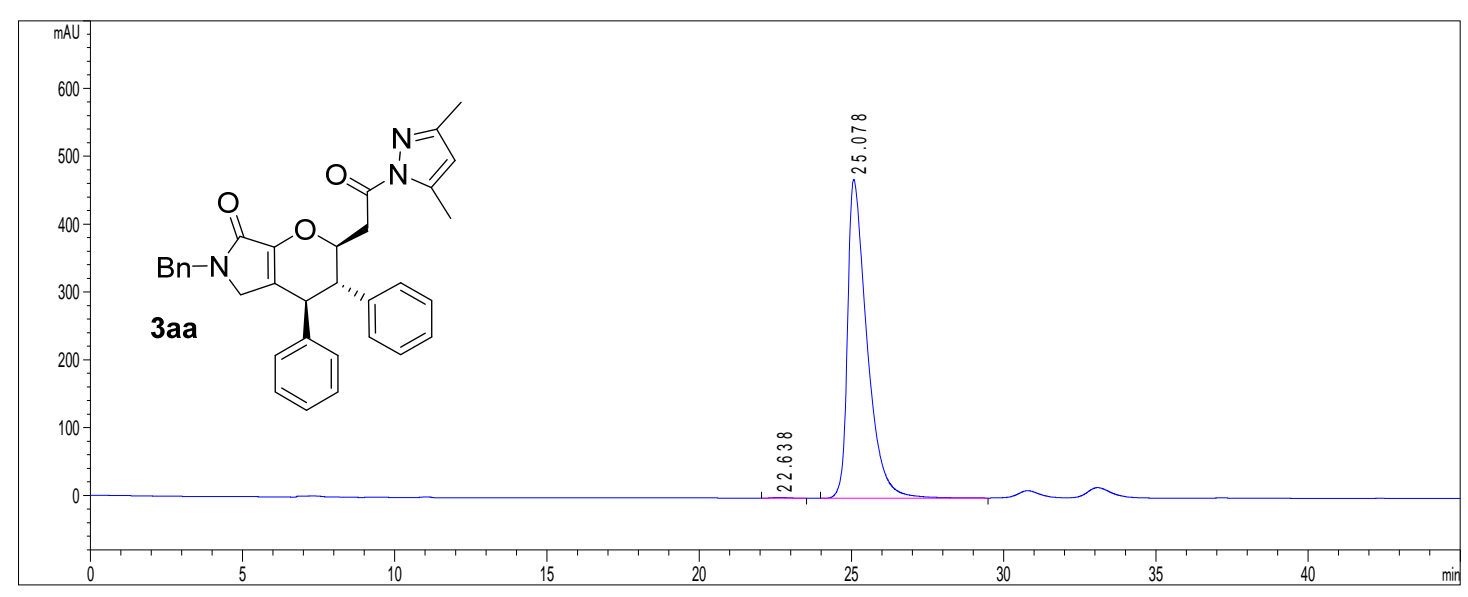

\begin{tabular}{|l|l|l|l|}
\hline & Retention & Area & Area\% \\
\hline 1 & 22.638 & 48.17403 & 0.2302 \\
\hline 2 & 25.078 & $2.08806 \mathrm{e} 4$ & 99.7698 \\
\hline
\end{tabular}




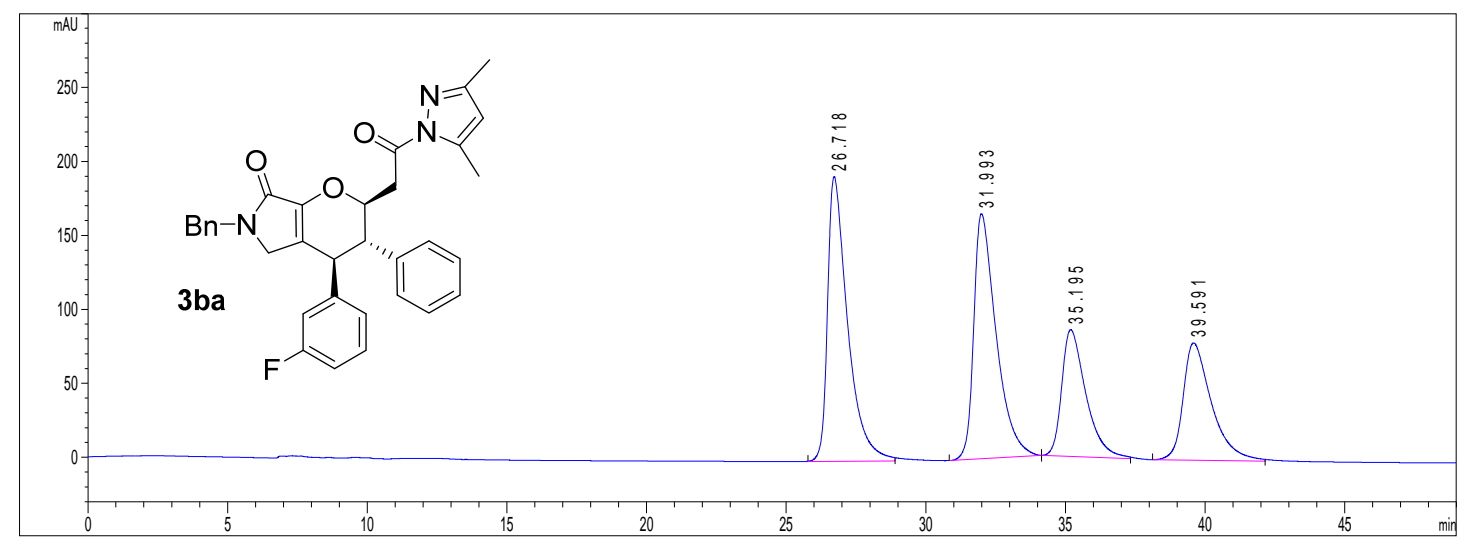

\begin{tabular}{|l|l|l|l|}
\hline & Retention & Area & Area\% \\
\hline 1 & 26.718 & 9621.29297 & 32.5012 \\
\hline 2 & 31.993 & 9225.67969 & 31.1648 \\
\hline 3 & 35.195 & 5156.69629 & 17.4196 \\
\hline 4 & 39.591 & 5599.17676 & 18.9143 \\
\hline
\end{tabular}

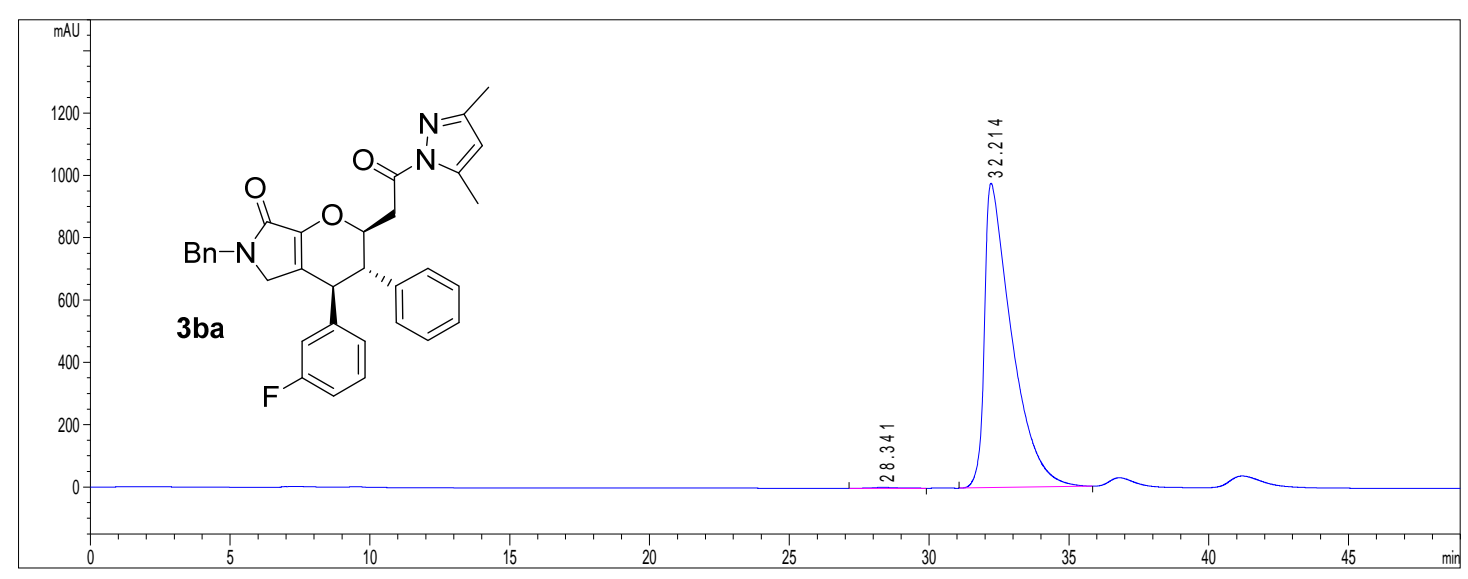

\begin{tabular}{|l|l|l|l|}
\hline & Retention & Area & Area\% \\
\hline 1 & 28.341 & 137.81410 & 0.2074 \\
\hline 2 & 32.214 & $6.63224 \mathrm{e} 4$ & 99.7926 \\
\hline
\end{tabular}




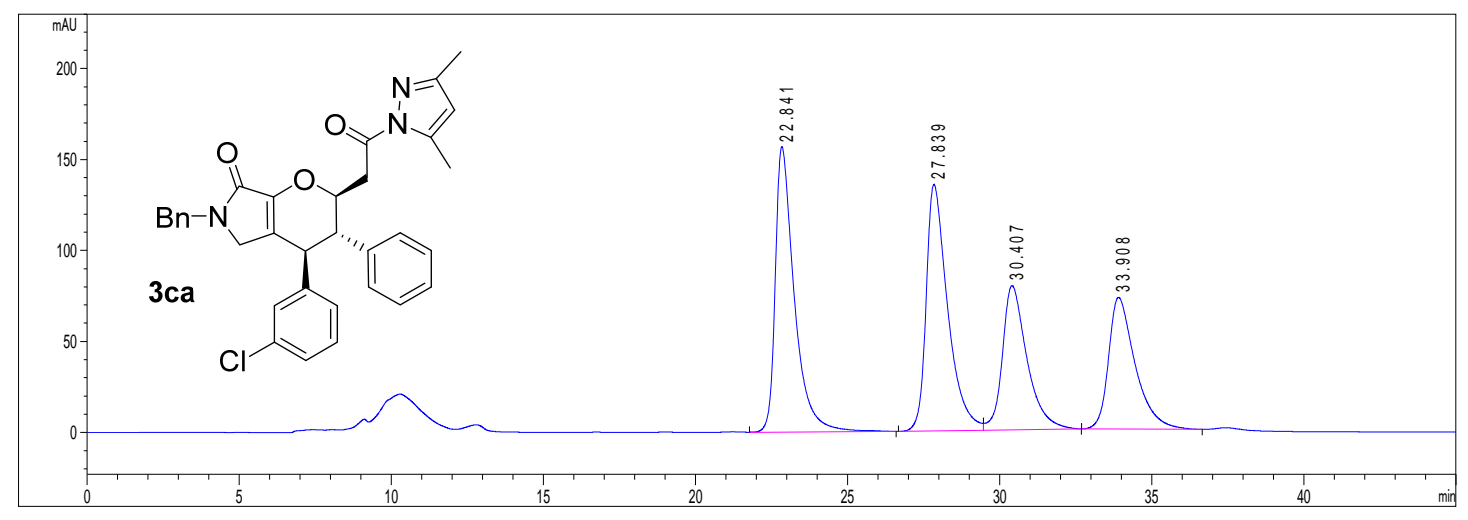

\begin{tabular}{|l|l|l|l|}
\hline & Retention & Area & Area\% \\
\hline 1 & 22.841 & 6825.42627 & 30.5127 \\
\hline 2 & 27.839 & 6682.35400 & 29.8731 \\
\hline 3 & 30.407 & 4459.74561 & 19.9371 \\
\hline 4 & 33.908 & 4401.57422 & 19.6770 \\
\hline
\end{tabular}

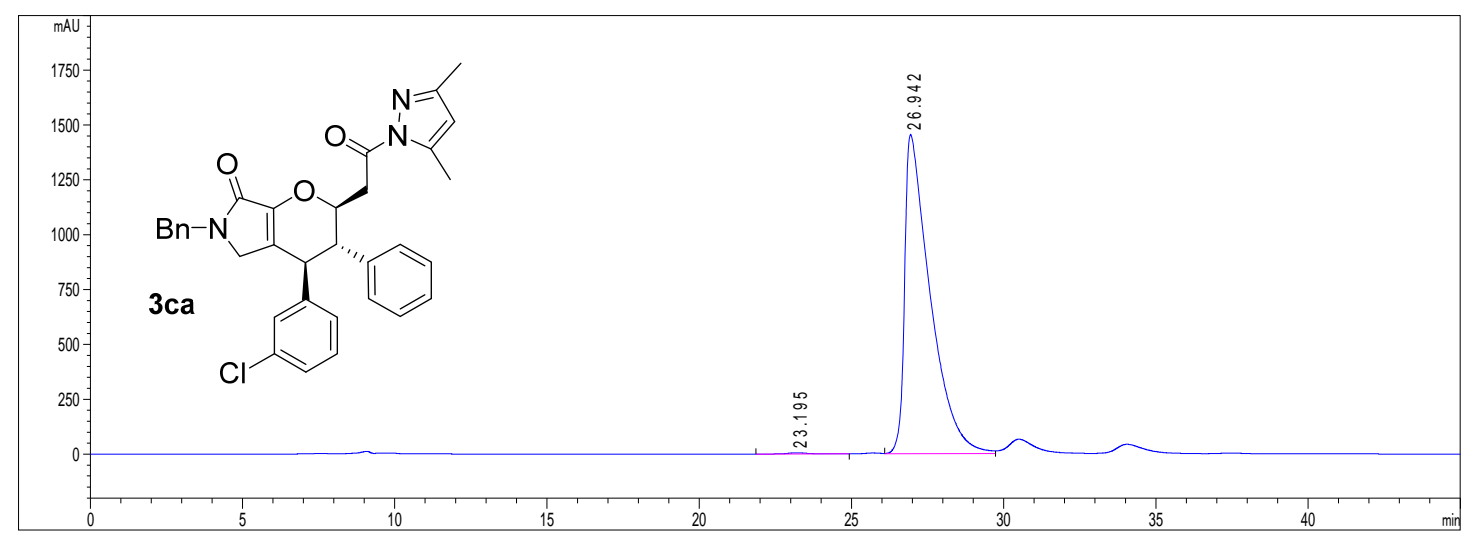

\begin{tabular}{|l|l|l|l|}
\hline & Retention & Area & Area $\%$ \\
\hline 1 & 23.195 & 239.61464 & 0.2812 \\
\hline 2 & 26.942 & $8.49663 \mathrm{e} 4$ & 99.7188 \\
\hline
\end{tabular}




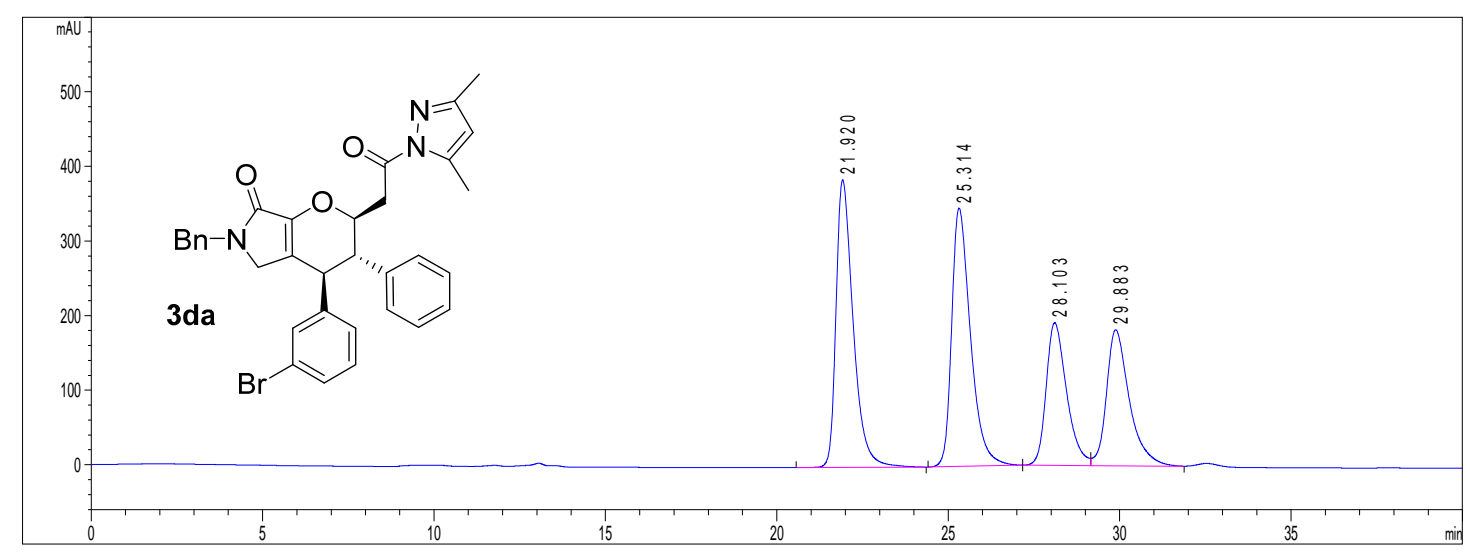

\begin{tabular}{|l|l|l|l|}
\hline & Retention & Area & Area\% \\
\hline 1 & 21.920 & $1.33731 \mathrm{e} 4$ & 31.1204 \\
\hline 2 & 25.314 & $1.31925 \mathrm{e} 4$ & 30.7001 \\
\hline 3 & 28.103 & 7940.77441 & 18.4788 \\
\hline 4 & 29.883 & 8465.82129 & 19.7007 \\
\hline
\end{tabular}

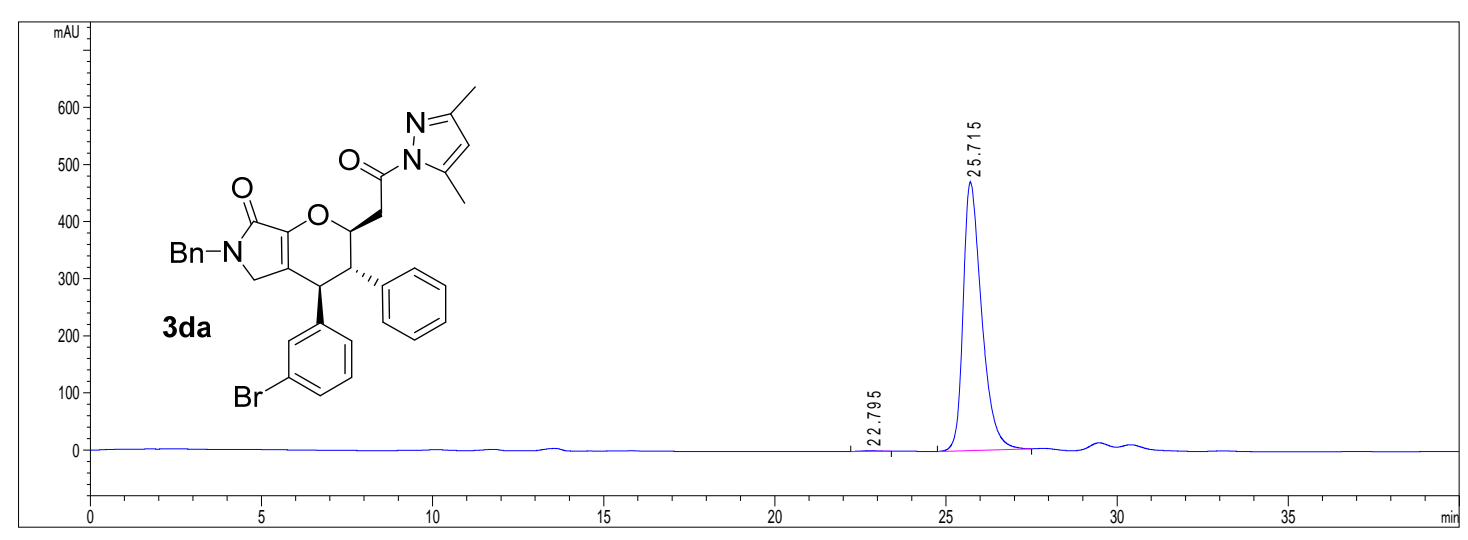

\begin{tabular}{|l|l|l|l|}
\hline & Retention & Area & Area\% \\
\hline 1 & 22.795 & 46.59922 & 0.2626 \\
\hline 2 & 25.715 & $1.76978 \mathrm{e} 4$ & 99.7374 \\
\hline
\end{tabular}




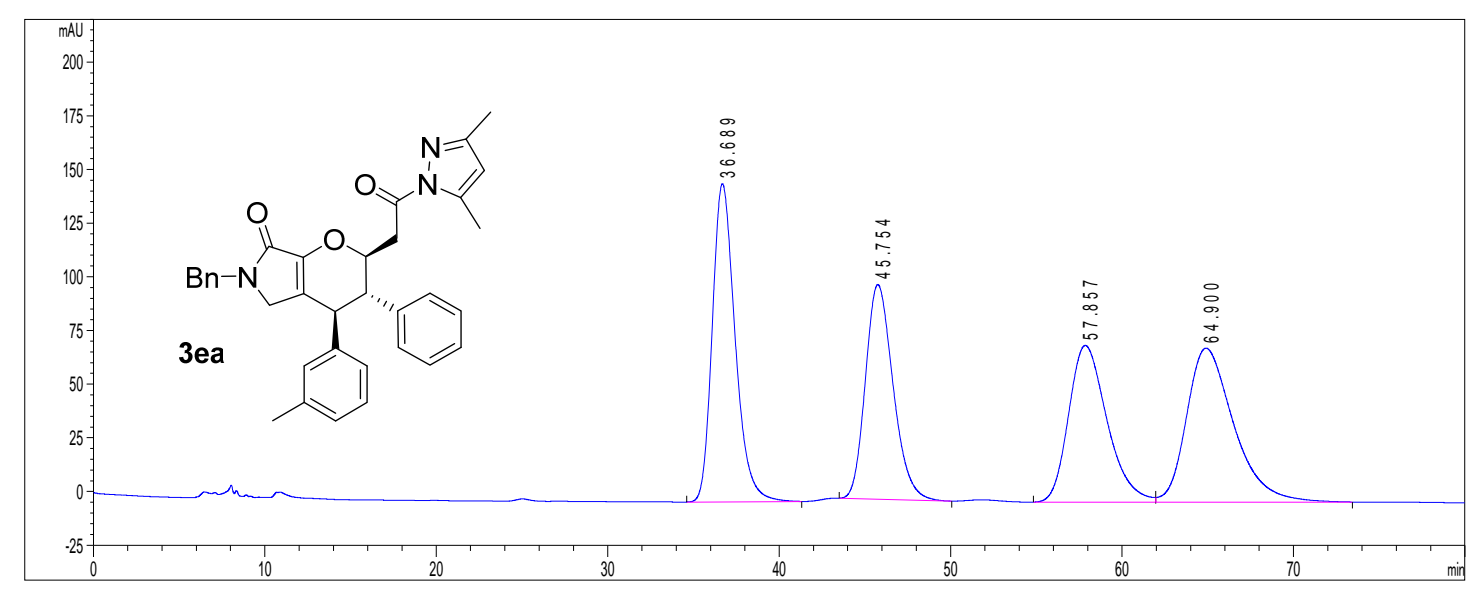

\begin{tabular}{|l|l|l|l|}
\hline & Retention & Area & Area\% \\
\hline 1 & 36.689 & $1.37217 \mathrm{e} 4$ & 27.3927 \\
\hline 2 & 45.754 & $1.12520 \mathrm{e} 4$ & 22.4624 \\
\hline 3 & 57.857 & $1.13927 \mathrm{e} 4$ & 22.7433 \\
\hline 4 & 64.900 & $1.37262 \mathrm{e} 4$ & 27.4017 \\
\hline
\end{tabular}

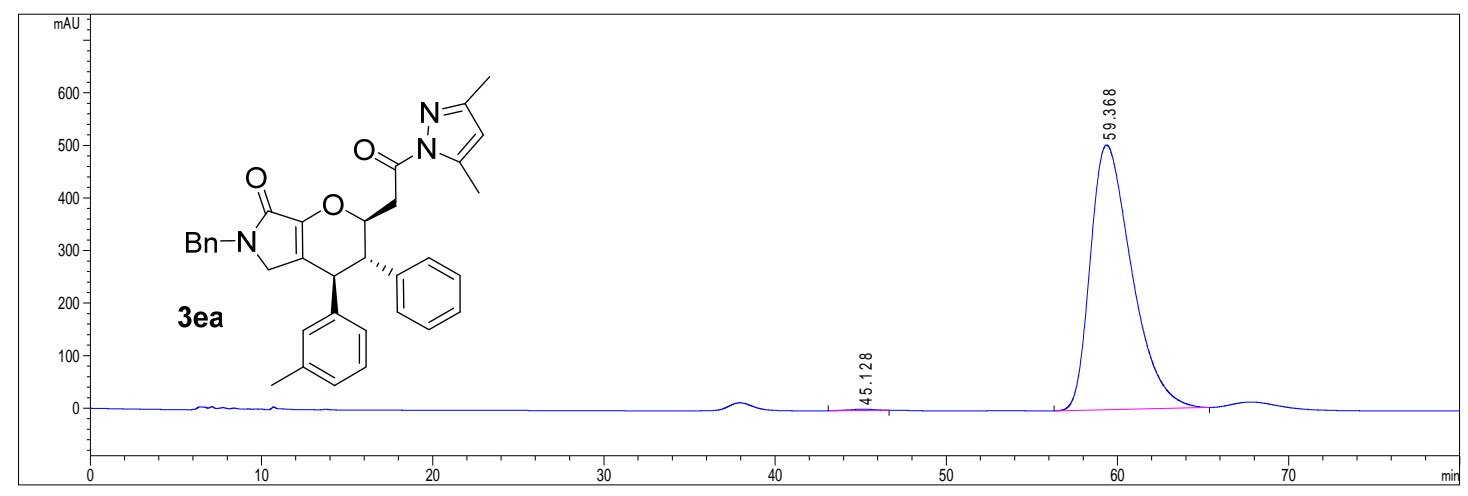

\begin{tabular}{|l|l|l|l|}
\hline & Retention & Area & Area\% \\
\hline 1 & 45.128 & 253.45882 & 0.2987 \\
\hline 2 & 59.368 & $8.46140 \mathrm{e} 4$ & 99.7013 \\
\hline
\end{tabular}




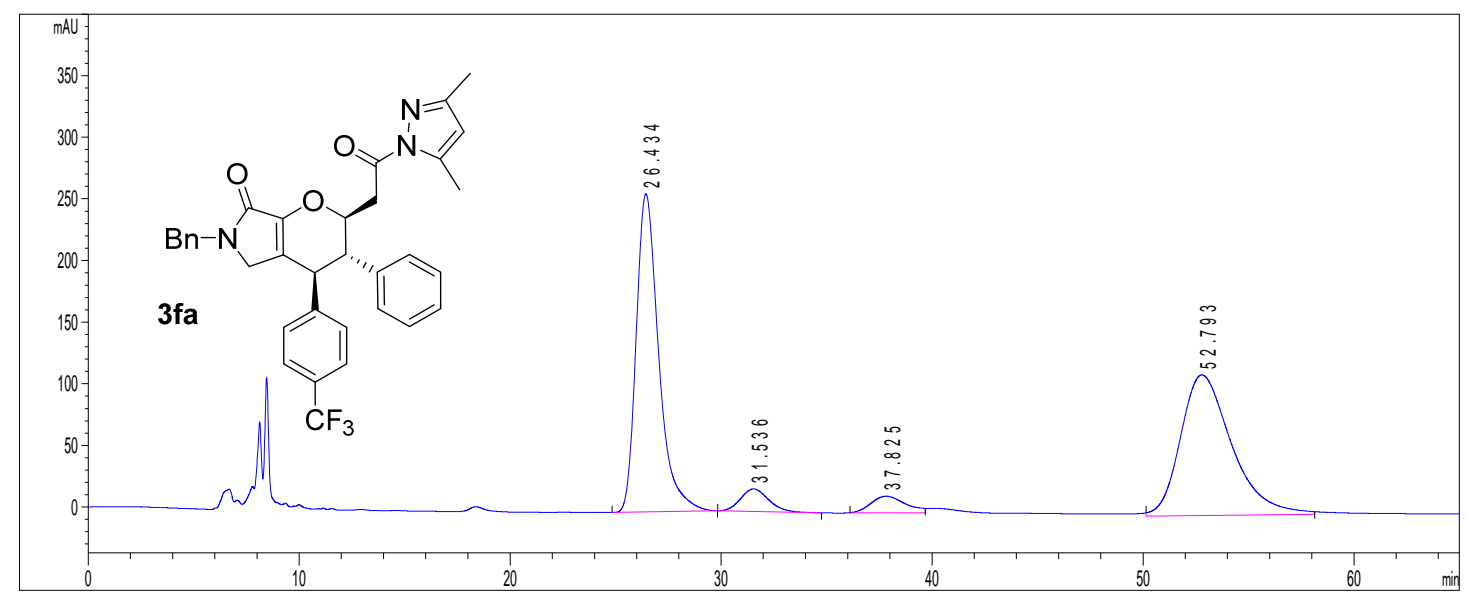

\begin{tabular}{|l|l|l|l|}
\hline & Retention & Area & Area\% \\
\hline 1 & 26.434 & $1.90809 \mathrm{e} 4$ & 45.9223 \\
\hline 2 & 31.536 & 1697.45215 & 4.0853 \\
\hline 3 & 37.825 & 1534.42664 & 3.6929 \\
\hline 4 & 52.793 & $1.92376 \mathrm{e} 4$ & 46.2995 \\
\hline
\end{tabular}

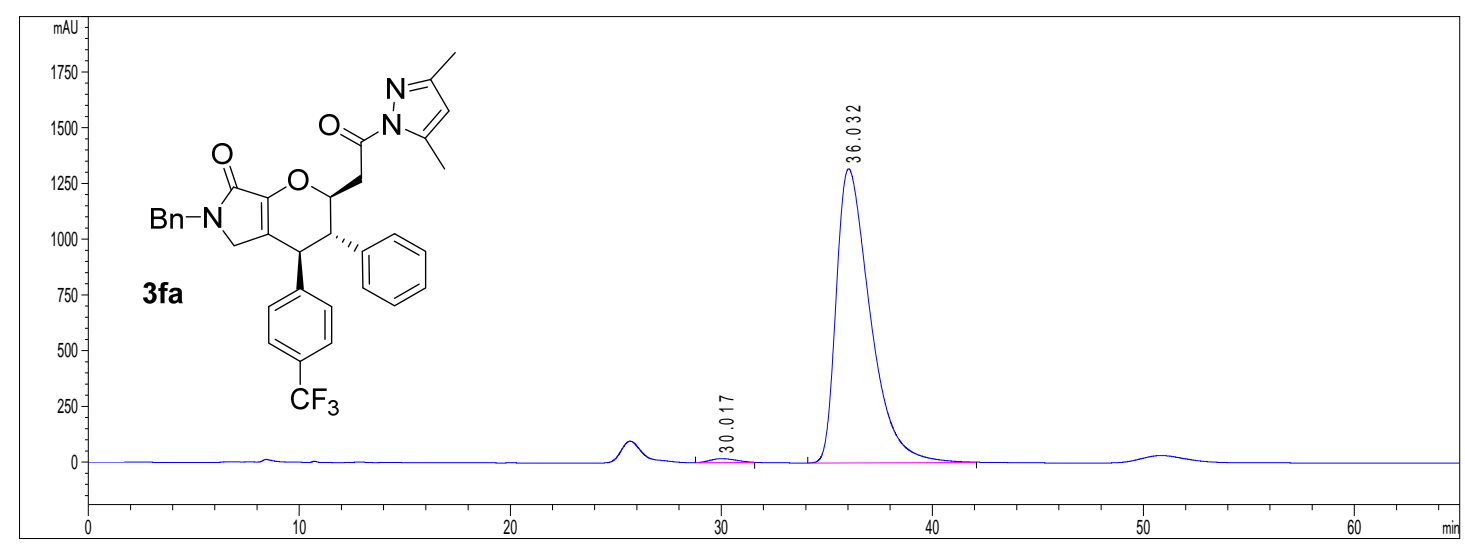

\begin{tabular}{|l|l|l|l|}
\hline & Retention & Area & Area\% \\
\hline 1 & 30.017 & 1587.34631 & 1.0625 \\
\hline 2 & 36.032 & $1.47814 \mathrm{e} 5$ & 98.9375 \\
\hline
\end{tabular}




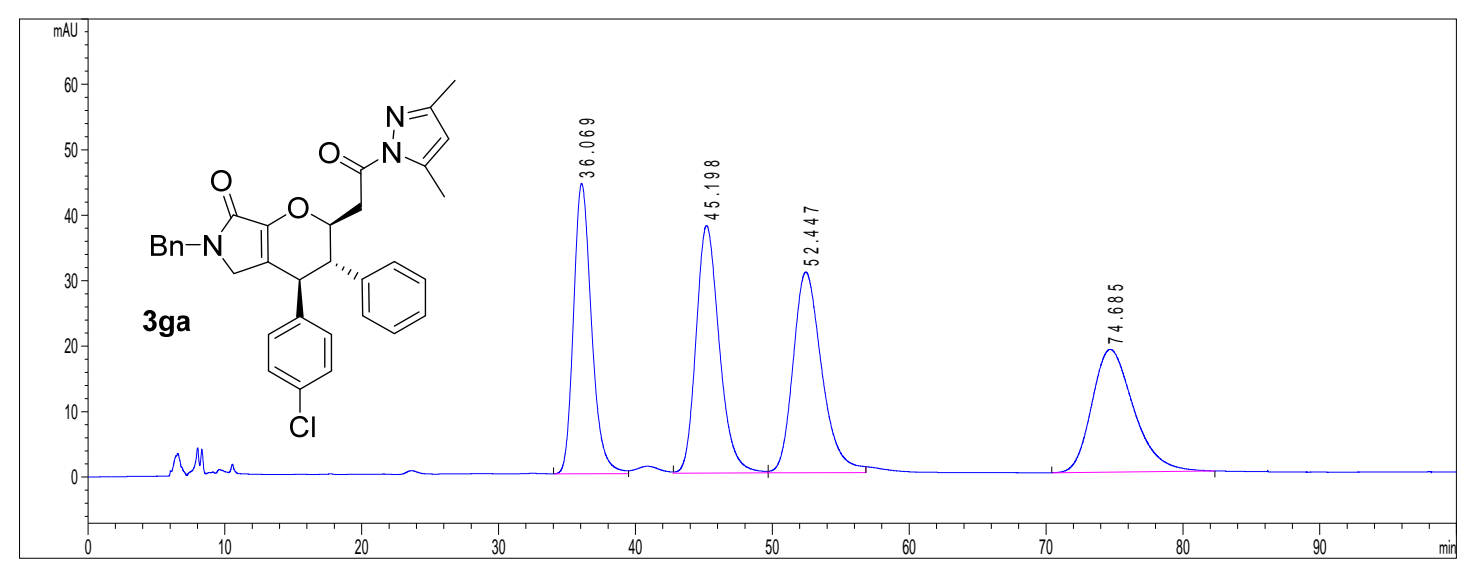

\begin{tabular}{|l|l|l|l|}
\hline & Retention & Area & Area\% \\
\hline 1 & 36.069 & 4059.38403 & 23.8946 \\
\hline 2 & 45.198 & 4476.46631 & 26.3497 \\
\hline 3 & 52.447 & 4480.50928 & 26.3735 \\
\hline 4 & 74.685 & 3972.35156 & 23.3823 \\
\hline
\end{tabular}

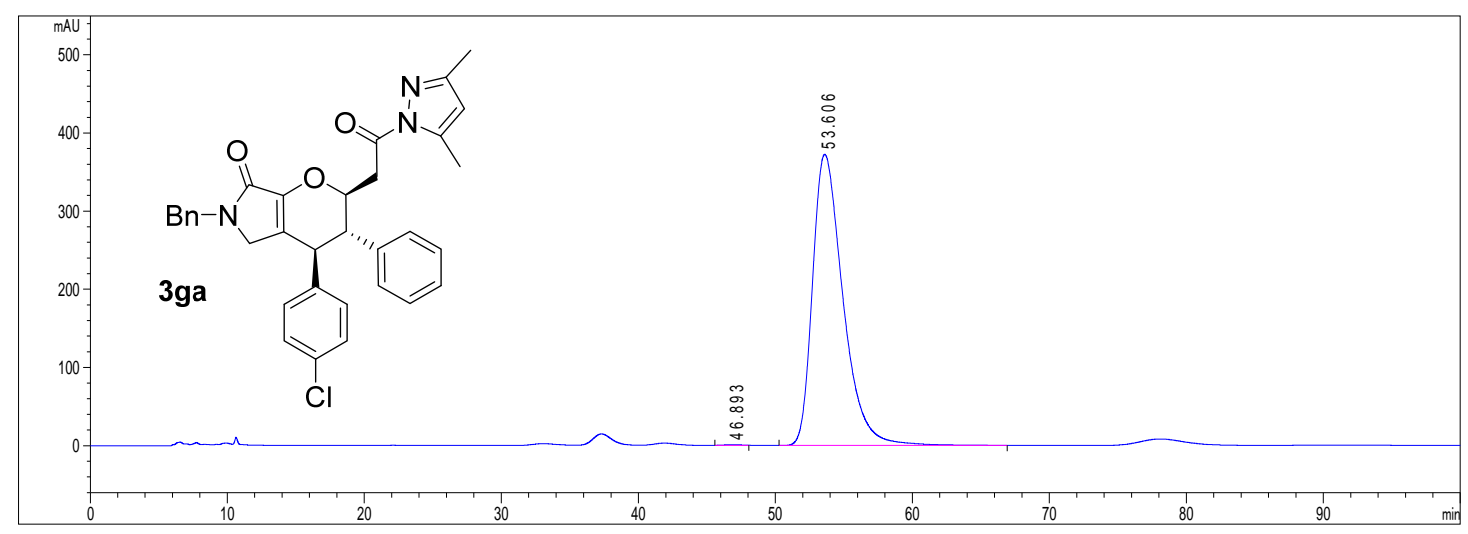

\begin{tabular}{|l|l|l|l|}
\hline & Retention & Area & Area\% \\
\hline 1 & 46.893 & 67.92425 & 0.1180 \\
\hline 2 & 53.606 & $5.74781 \mathrm{e} 4$ & 100.0000 \\
\hline
\end{tabular}




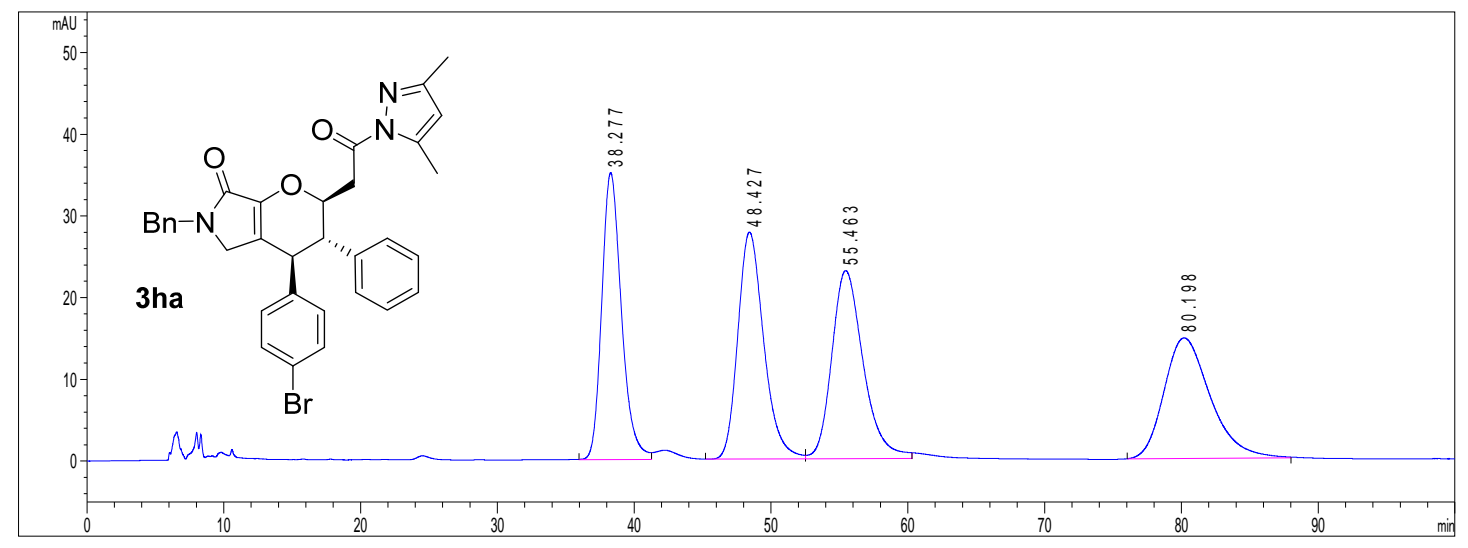

\begin{tabular}{|l|l|l|l|}
\hline & Retention & Area & Area\% \\
\hline 1 & 38.277 & 3510.63599 & 24.4984 \\
\hline 2 & 48.427 & 3658.44629 & 25.5299 \\
\hline 3 & 55.463 & 3690.68213 & 25.7549 \\
\hline 4 & 80.198 & 3470.27344 & 24.2168 \\
\hline
\end{tabular}

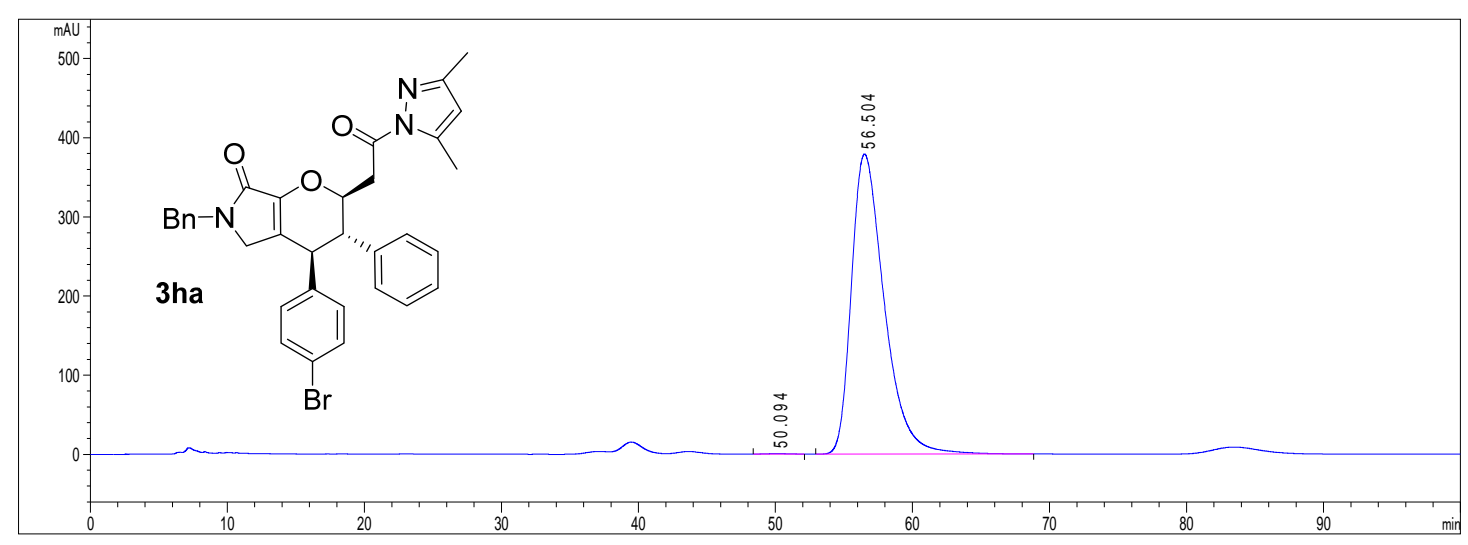

\begin{tabular}{|l|l|l|l|}
\hline & Retention & Area & Area\% \\
\hline 1 & 50.094 & 92.46234 & 0.1460 \\
\hline 2 & 56.504 & $6.32313 \mathrm{e} 4$ & 99.8540 \\
\hline
\end{tabular}




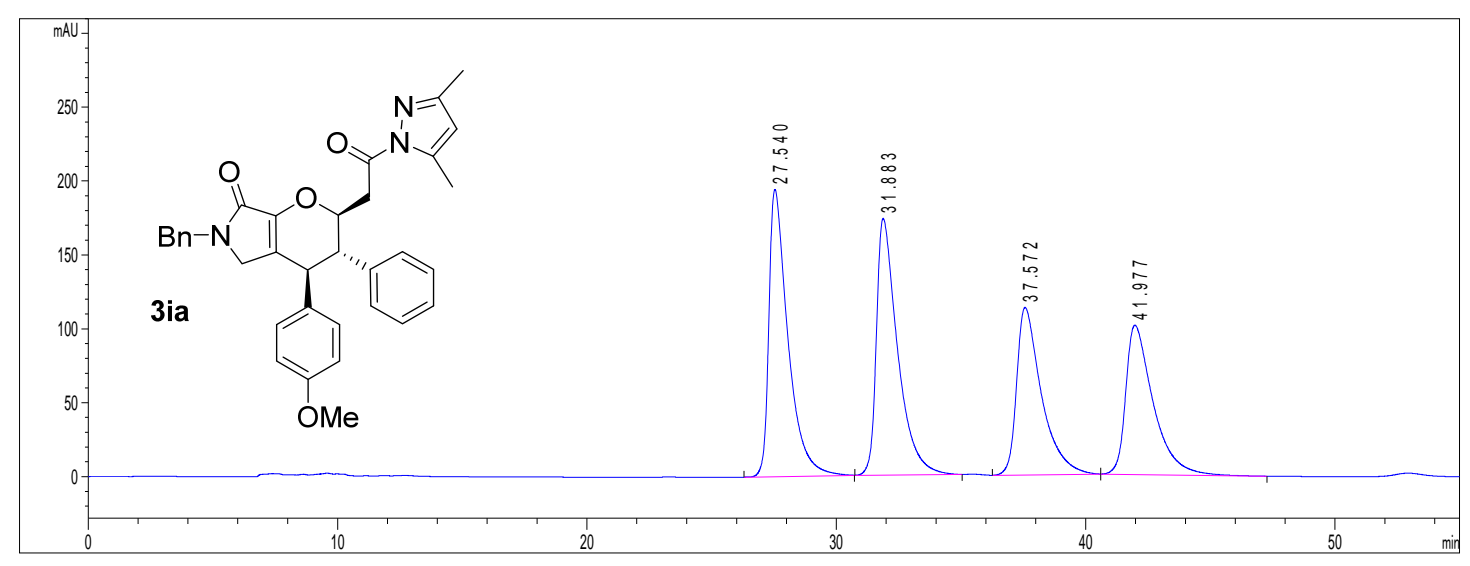

\begin{tabular}{|l|l|l|l|}
\hline & Retention & Area & Area\% \\
\hline 1 & 27.540 & $1.03244 \mathrm{e} 4$ & 28.5916 \\
\hline 2 & 31.883 & $1.02215 \mathrm{e} 4$ & 28.3065 \\
\hline 3 & 37.572 & 7840.47412 & 21.7127 \\
\hline 4 & 41.977 & 7723.68115 & 21.3892 \\
\hline
\end{tabular}

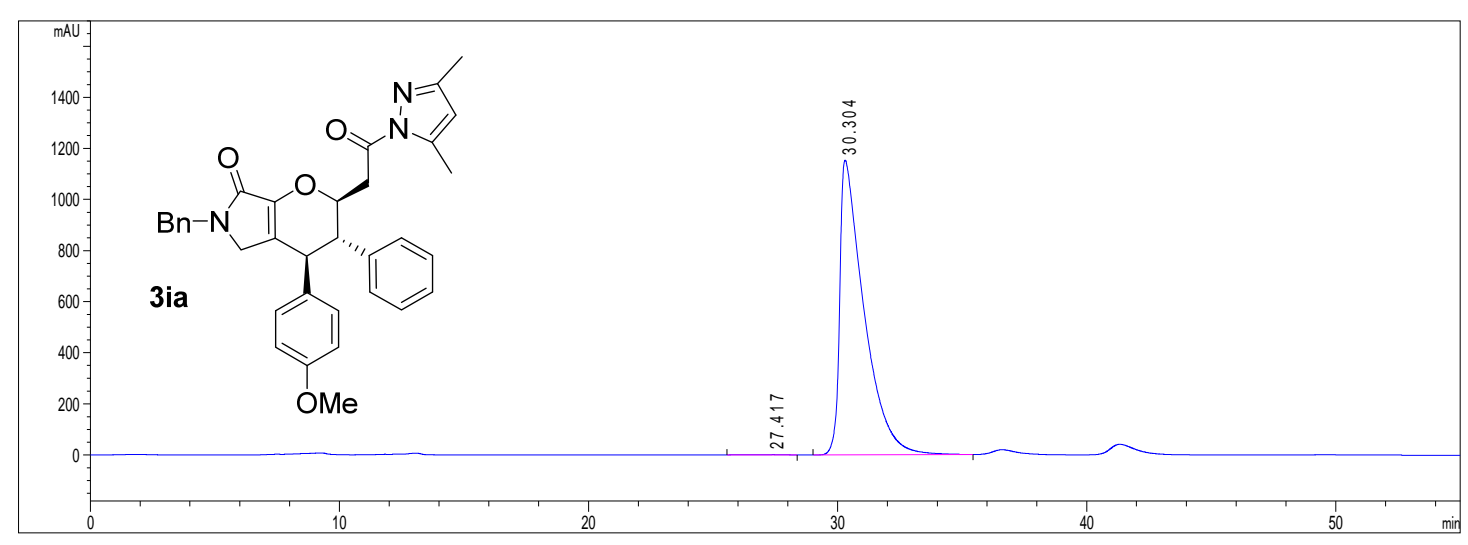

\begin{tabular}{|l|l|l|l|}
\hline & Retention & Area & Area\% \\
\hline 1 & 27.417 & 157.37483 & 0.2012 \\
\hline 2 & 30.304 & $7.80650 \mathrm{e} 4$ & 99.7988 \\
\hline
\end{tabular}




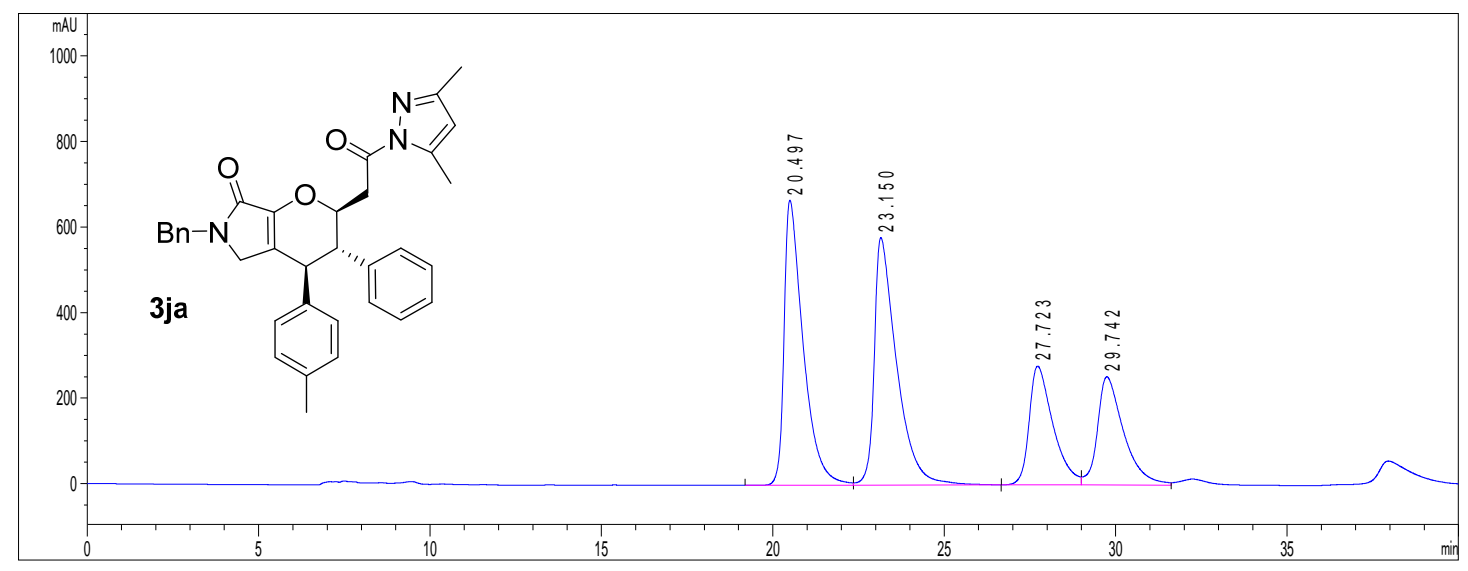

\begin{tabular}{|l|l|l|l|}
\hline & Retention & Area & Area $\%$ \\
\hline 1 & 20.497 & $2.58207 \mathrm{e} 4$ & 32.6263 \\
\hline 2 & 23.150 & $2.63160 \mathrm{e} 4$ & 33.2521 \\
\hline 3 & 27.723 & $1.34735 \mathrm{e} 4$ & 17.0247 \\
\hline 4 & 29.742 & $1.35305 \mathrm{e} 4$ & 17.0968 \\
\hline
\end{tabular}

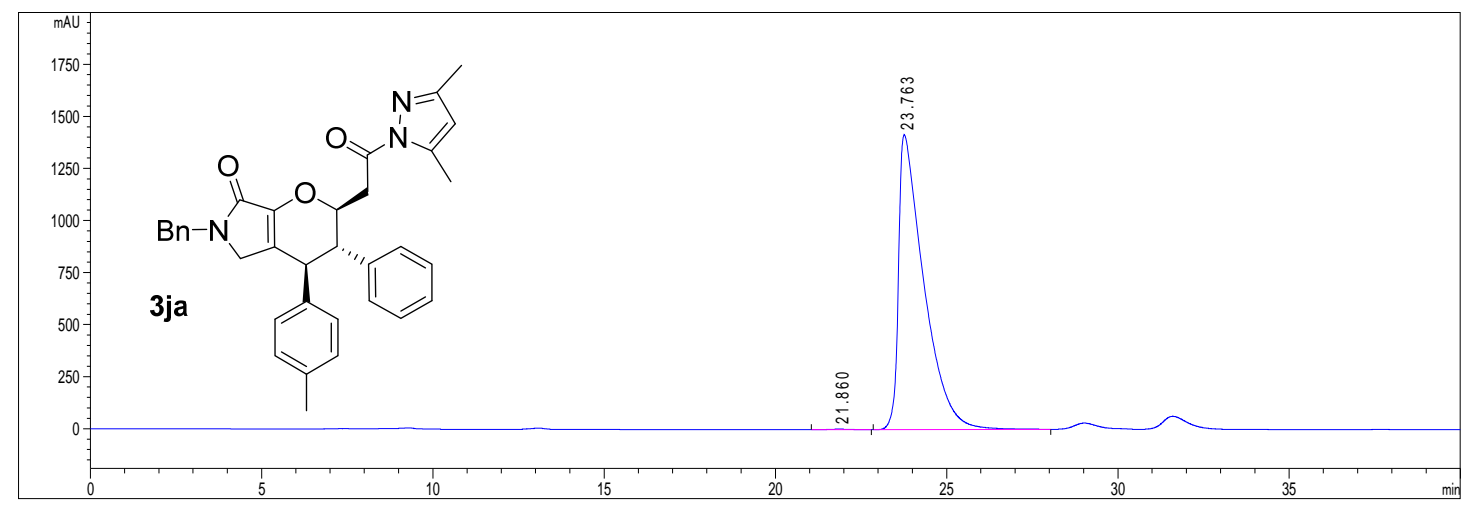

\begin{tabular}{|l|l|l|l|}
\hline & Retention & Area & Area $\%$ \\
\hline 1 & 21.860 & 86.07483 & 0.1183 \\
\hline 2 & 23.763 & $7.26519 \mathrm{e} 4$ & 99.8817 \\
\hline
\end{tabular}




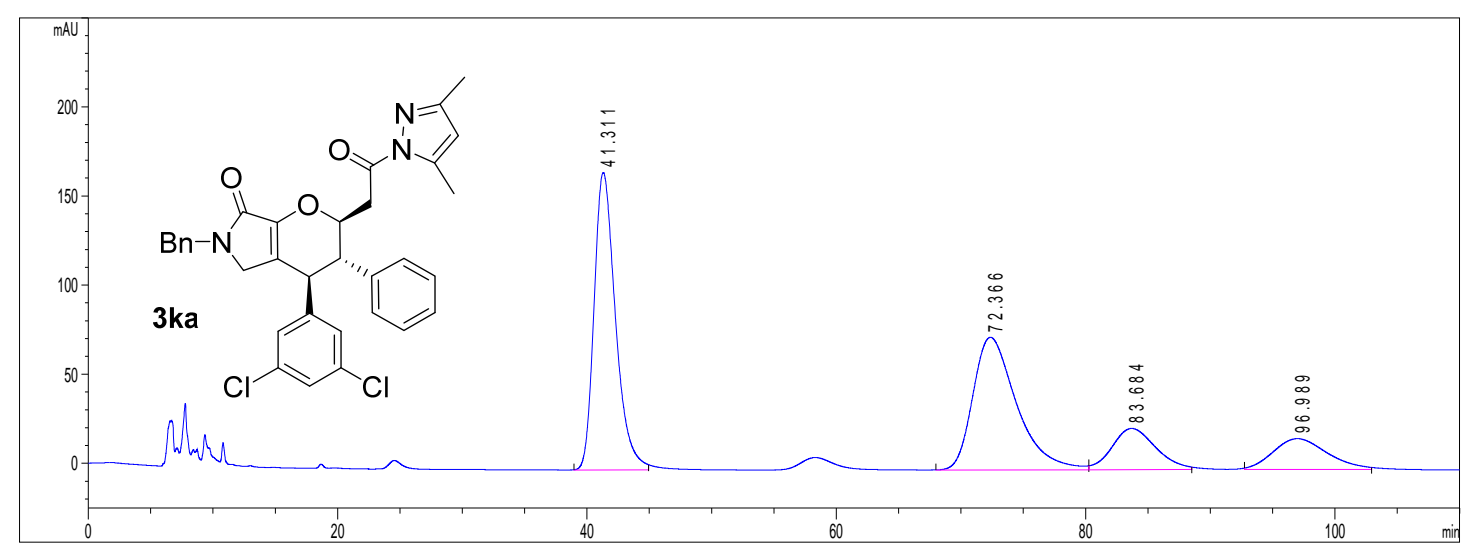

\begin{tabular}{|l|l|l|l|}
\hline & Retention & Area & Area\% \\
\hline 1 & 41.311 & $1.98522 \mathrm{e} 4$ & 40.6642 \\
\hline 2 & 72.366 & $1.81304 \mathrm{e} 4$ & 37.1374 \\
\hline 3 & 83.684 & 5625.40234 & 11.5228 \\
\hline 4 & 96.989 & 5211.79150 & 10.6756 \\
\hline
\end{tabular}

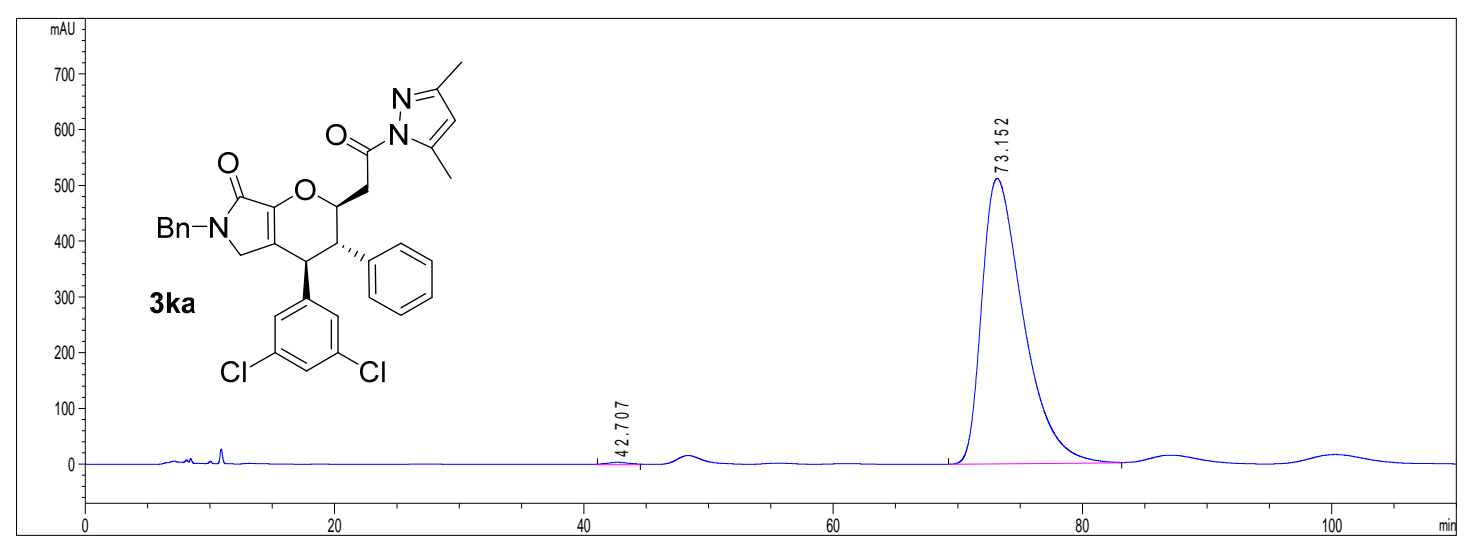

\begin{tabular}{|l|l|l|l|}
\hline & Retention & Area & Area\% \\
\hline 1 & 42.707 & 523.30206 & 0.4390 \\
\hline 2 & 73.152 & $1.18673 \mathrm{e} 5$ & 99.5610 \\
\hline
\end{tabular}




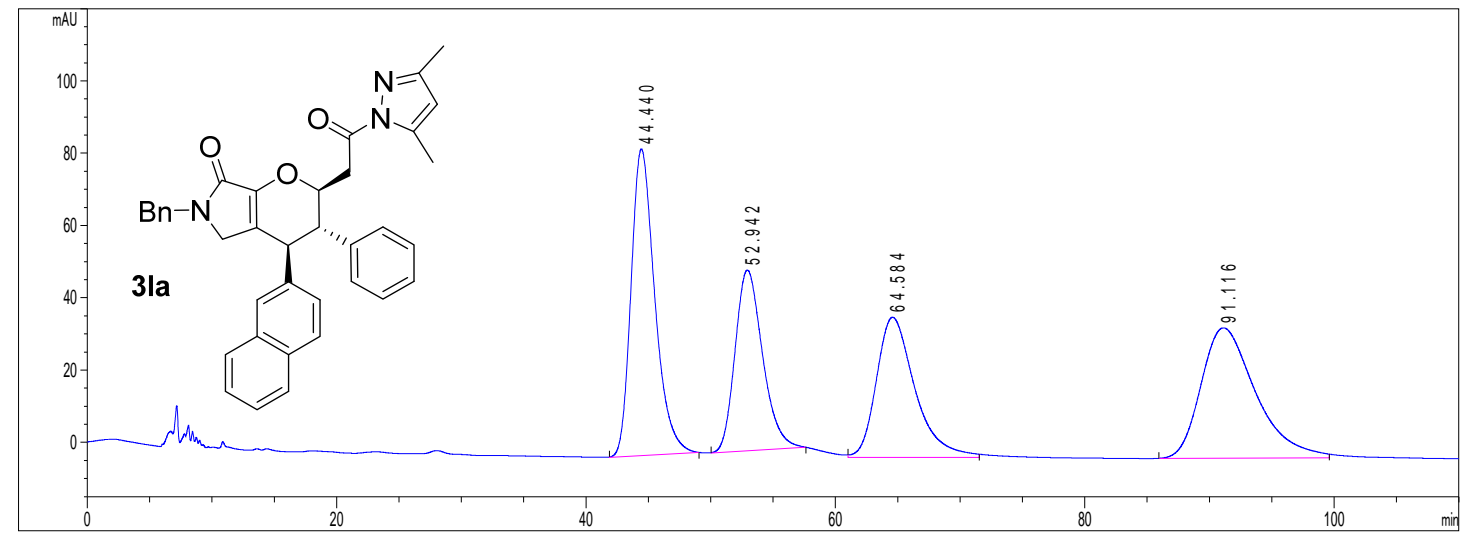

\begin{tabular}{|l|l|l|l|}
\hline & Retention & Area & Area $\%$ \\
\hline 1 & 44.440 & $1.11985 \mathrm{e} 4$ & 29.3532 \\
\hline 2 & 52.942 & 7661.40527 & 20.0818 \\
\hline 3 & 64.584 & 8331.09766 & 21.8372 \\
\hline 4 & 91.116 & $1.09599 \mathrm{e} 4$ & 28.7277 \\
\hline
\end{tabular}

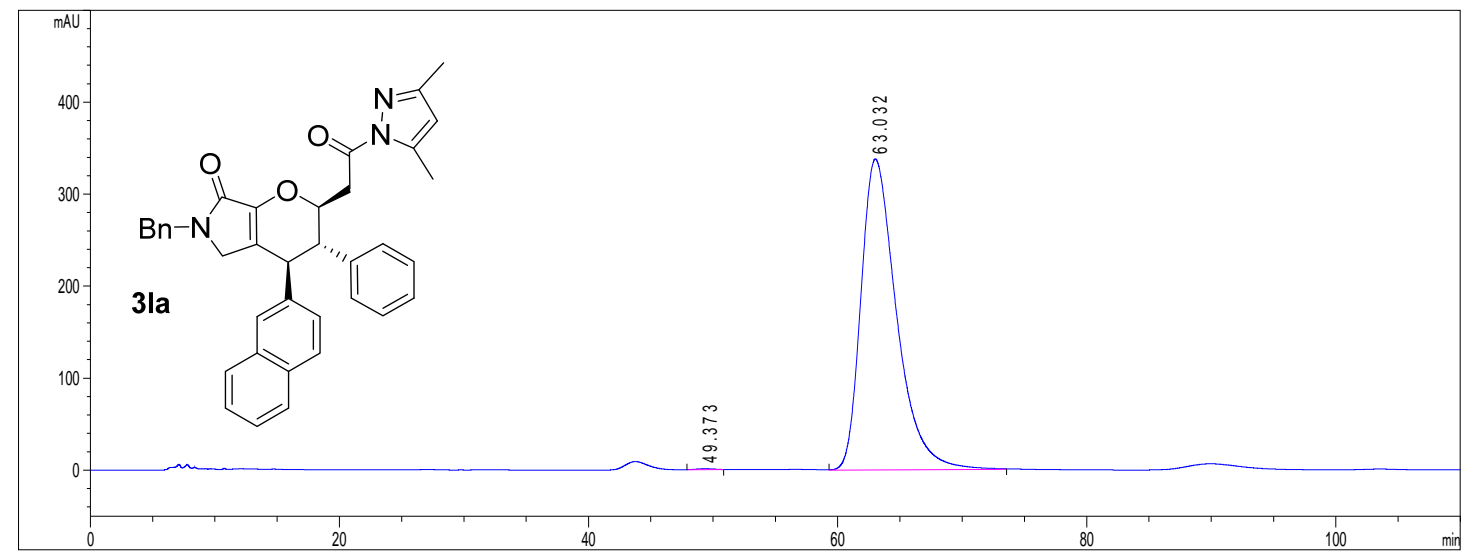

\begin{tabular}{|l|l|l|l|}
\hline & Retention & Area & Area\% \\
\hline 1 & 49.373 & 96.51254 & 0.1436 \\
\hline 2 & 63.032 & $6.70980 \mathrm{e} 4$ & 99.8564 \\
\hline
\end{tabular}




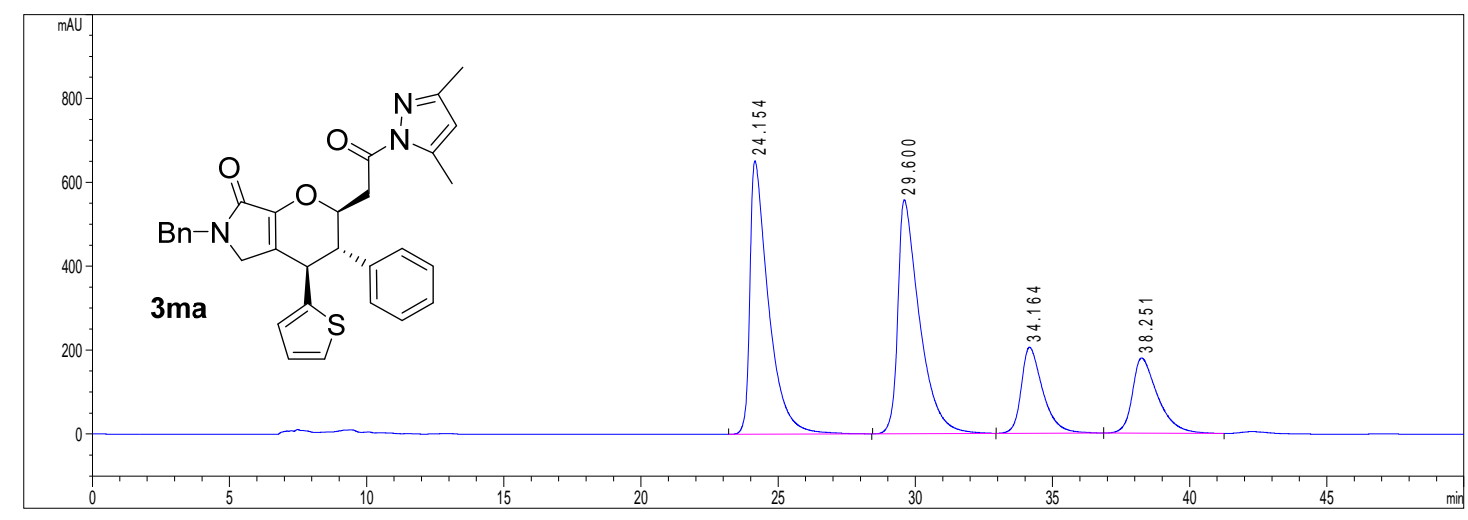

\begin{tabular}{|l|l|l|l|}
\hline & Retention & Area & Area\% \\
\hline 1 & 24.154 & $3.07547 \mathrm{e} 4$ & 36.6127 \\
\hline 2 & 29.600 & $3.06208 \mathrm{e} 4$ & 36.4533 \\
\hline 3 & 34.164 & $1.11520 \mathrm{e} 4$ & 13.2761 \\
\hline 4 & 38.251 & $1.14725 \mathrm{e} 4$ & 13.6578 \\
\hline
\end{tabular}

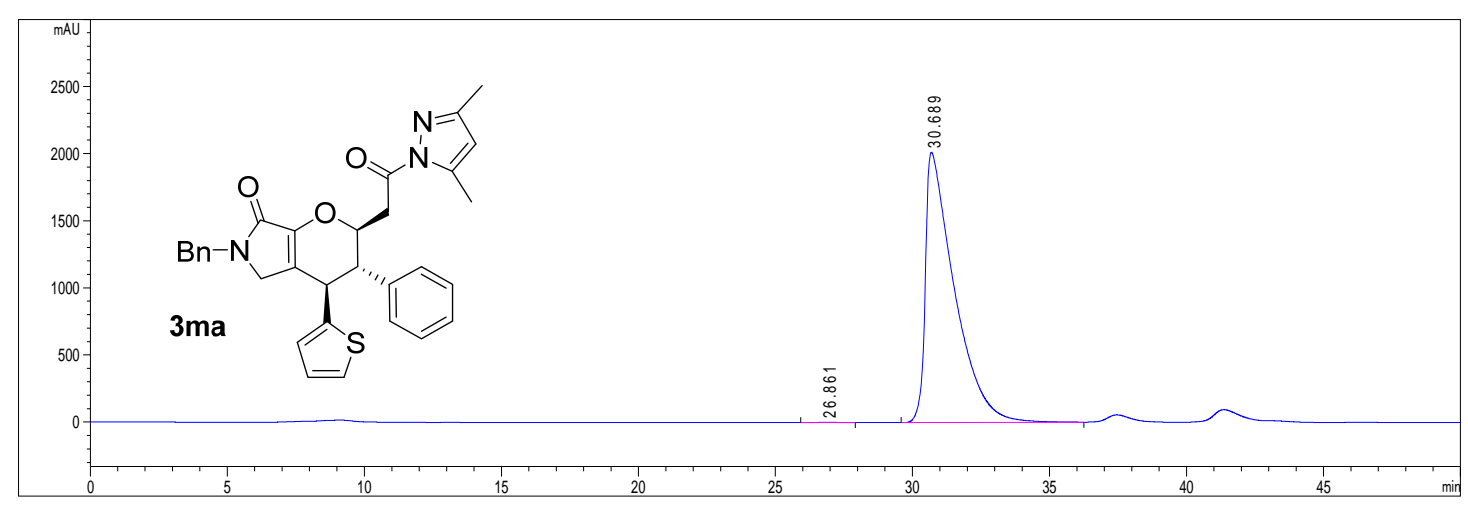

\begin{tabular}{|l|l|l|l|}
\hline & Retention & Area & Area $\%$ \\
\hline 1 & 26.861 & 113.65583 & 0.0767 \\
\hline 2 & 30.689 & $1.48018 \mathrm{e} 5$ & 99.9233 \\
\hline
\end{tabular}




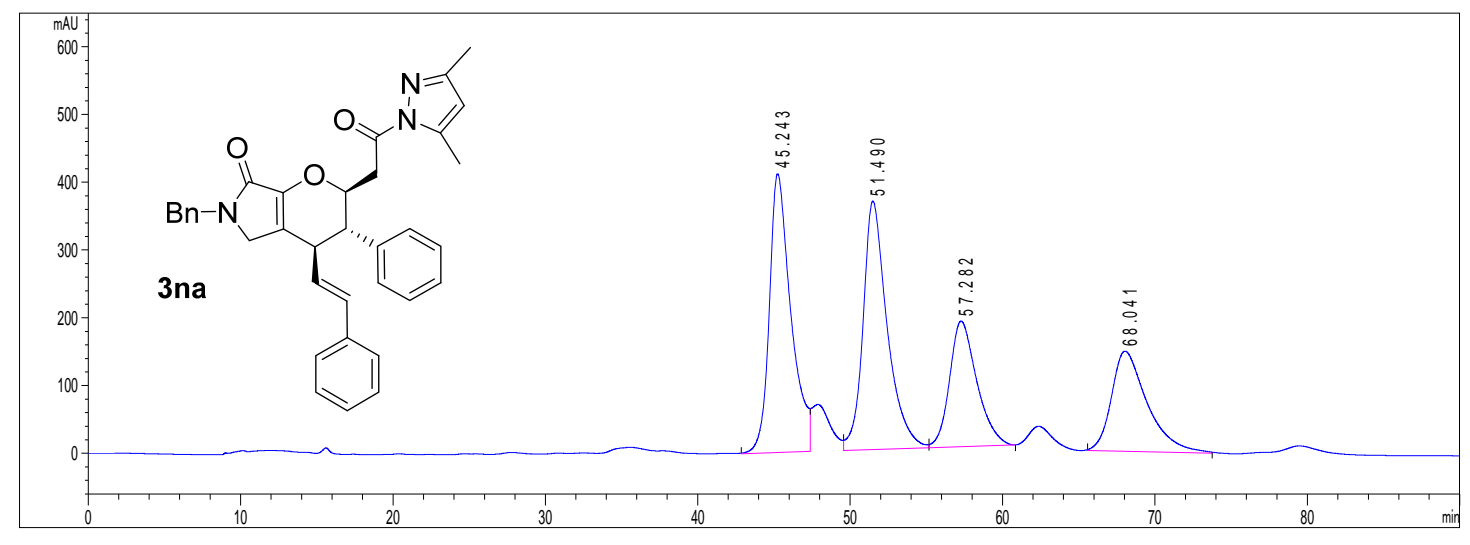

\begin{tabular}{|l|l|l|l|}
\hline & Retention & Area & Area\% \\
\hline 1 & 45.243 & $4.04659 \mathrm{e} 4$ & 31.5942 \\
\hline 2 & 51.490 & $4.04510 \mathrm{e} 4$ & 31.5825 \\
\hline 3 & 57.282 & $2.30765 \mathrm{e} 4$ & 18.0172 \\
\hline 4 & 68.041 & $2.40869 \mathrm{e} 4$ & 18.8061 \\
\hline
\end{tabular}

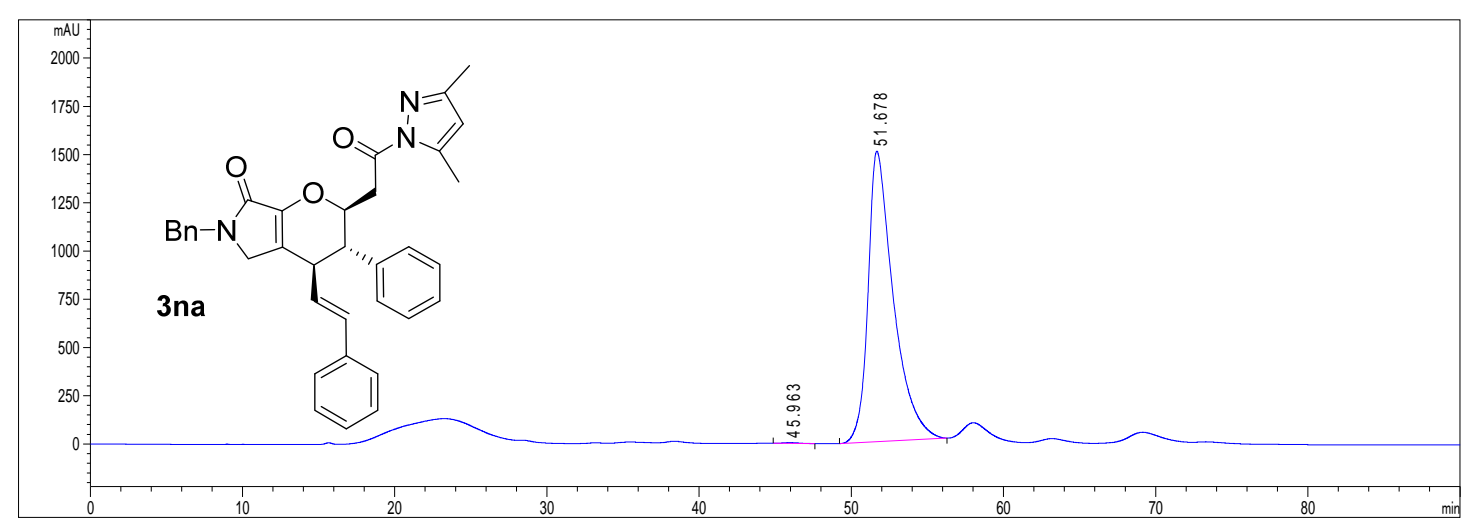

\begin{tabular}{|l|l|l|l|}
\hline & Retention & Area & Area $\%$ \\
\hline 1 & 45.963 & 287.28714 & 0.1644 \\
\hline 2 & 51.678 & $1.74492 \mathrm{e} 5$ & 99.8356 \\
\hline
\end{tabular}




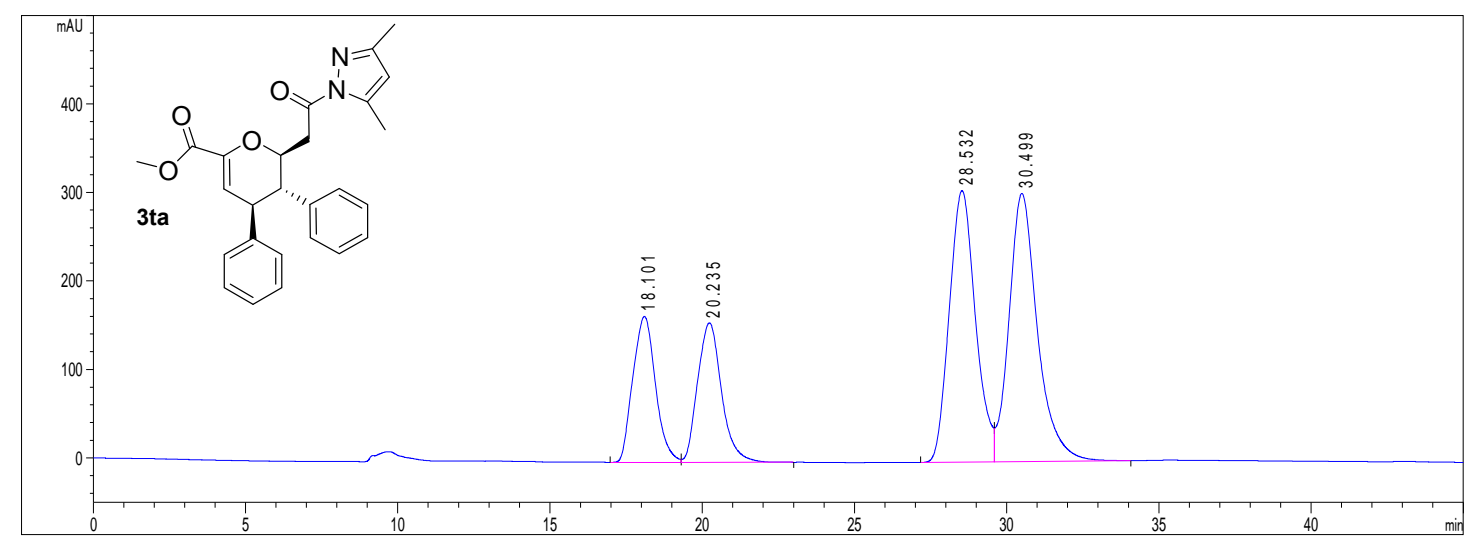

\begin{tabular}{|l|l|l|l|}
\hline & Retention & Area & Area\% \\
\hline 1 & 18.101 & 8521.61426 & 15.4913 \\
\hline 2 & 20.235 & 8604.24219 & 15.6415 \\
\hline 3 & 28.532 & $1.82903 \mathrm{e} 4$ & 33.2496 \\
\hline 4 & 30.499 & $1.95930 \mathrm{e} 4$ & 35.6177 \\
\hline
\end{tabular}

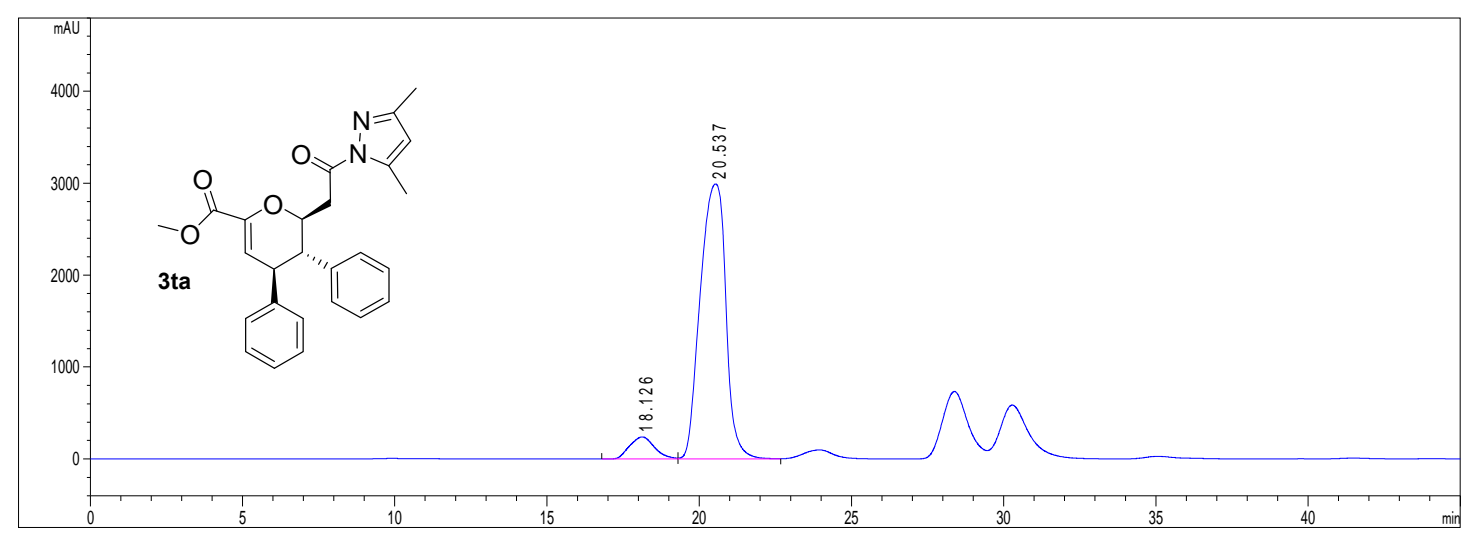

\begin{tabular}{|l|l|l|l|}
\hline & Retention & Area & Area $\%$ \\
\hline 1 & 18.126 & $1.34957 \mathrm{e} 4$ & 7.1815 \\
\hline 2 & 20.537 & $1.74427 \mathrm{e} 5$ & 92.8185 \\
\hline
\end{tabular}




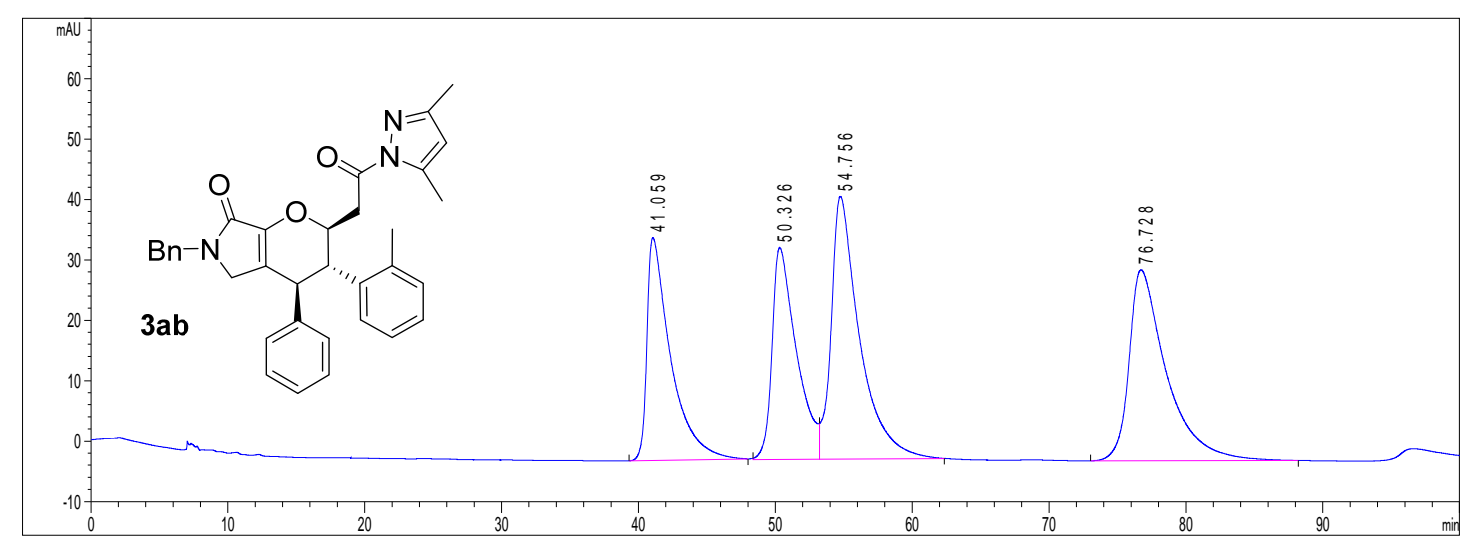

\begin{tabular}{|l|l|l|l|}
\hline & Retention & Area & Area\% \\
\hline 1 & 41.059 & 4433.17188 & 21.0482 \\
\hline 2 & 50.326 & 4359.98145 & 20.7007 \\
\hline 3 & 54.756 & 6283.05859 & 29.8312 \\
\hline 4 & 76.728 & 5985.80811 & 28.4199 \\
\hline
\end{tabular}

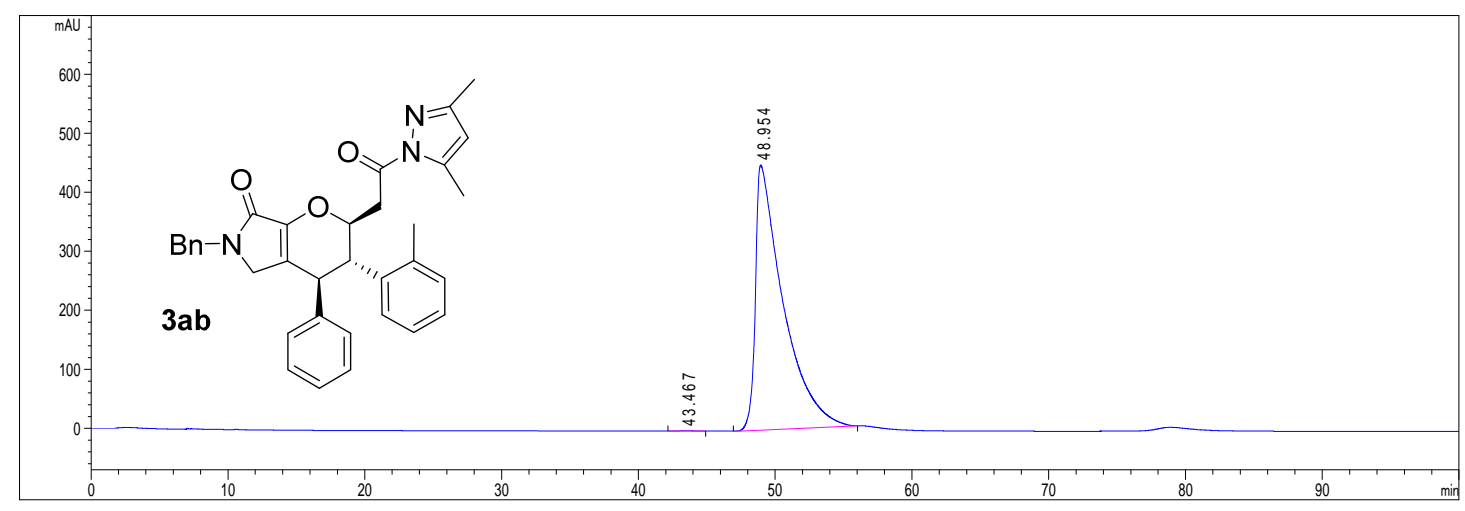

\begin{tabular}{|l|l|l|l|}
\hline & Retention & Area & Area\% \\
\hline 1 & 43.467 & 37.80748 & 0.0597 \\
\hline 2 & 48.954 & $6.32452 \mathrm{e} 4$ & 99.9403 \\
\hline
\end{tabular}




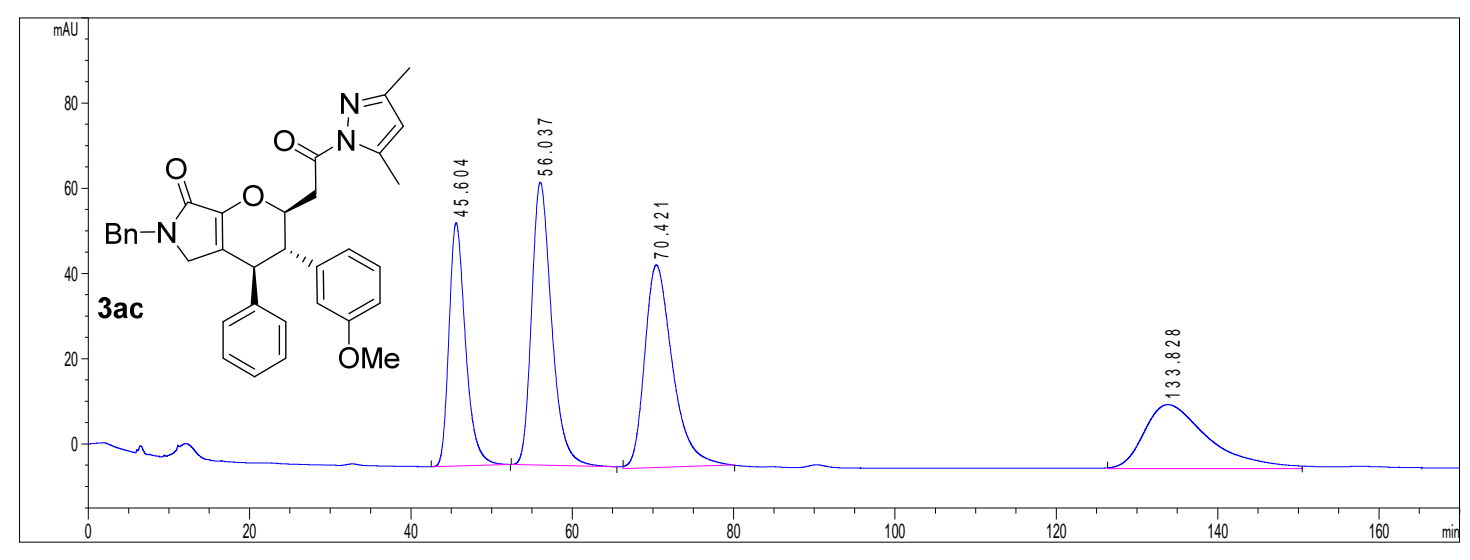

\begin{tabular}{|l|l|l|l|}
\hline & Retention & Area & Area\% \\
\hline 1 & 45.604 & 8107.08887 & 20.6789 \\
\hline 2 & 56.037 & $1.14367 \mathrm{e} 4$ & 29.1718 \\
\hline 3 & 70.421 & $1.14131 \mathrm{e} 4$ & 29.1115 \\
\hline 4 & 133.828 & 8247.84082 & 21.0379 \\
\hline
\end{tabular}

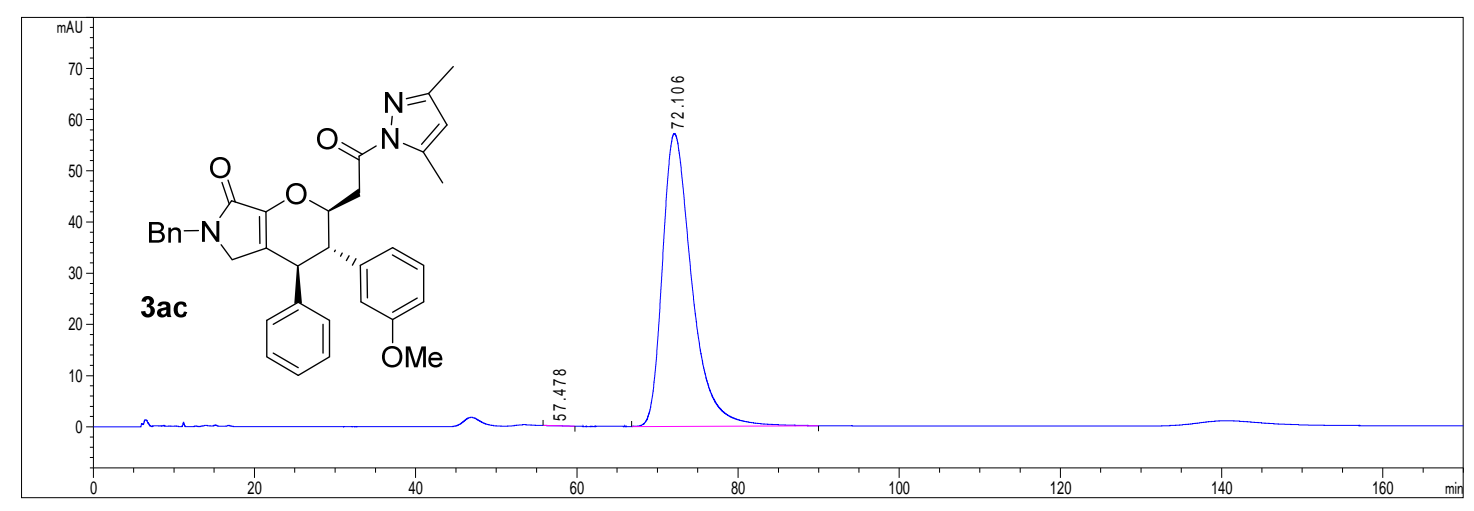

\begin{tabular}{|l|l|l|l|}
\hline & Retention & Area & Area\% \\
\hline 1 & 57.478 & 5.75580 & 0.0396 \\
\hline 2 & 72.106 & $1.45323 \mathrm{e} 4$ & 99.9604 \\
\hline
\end{tabular}




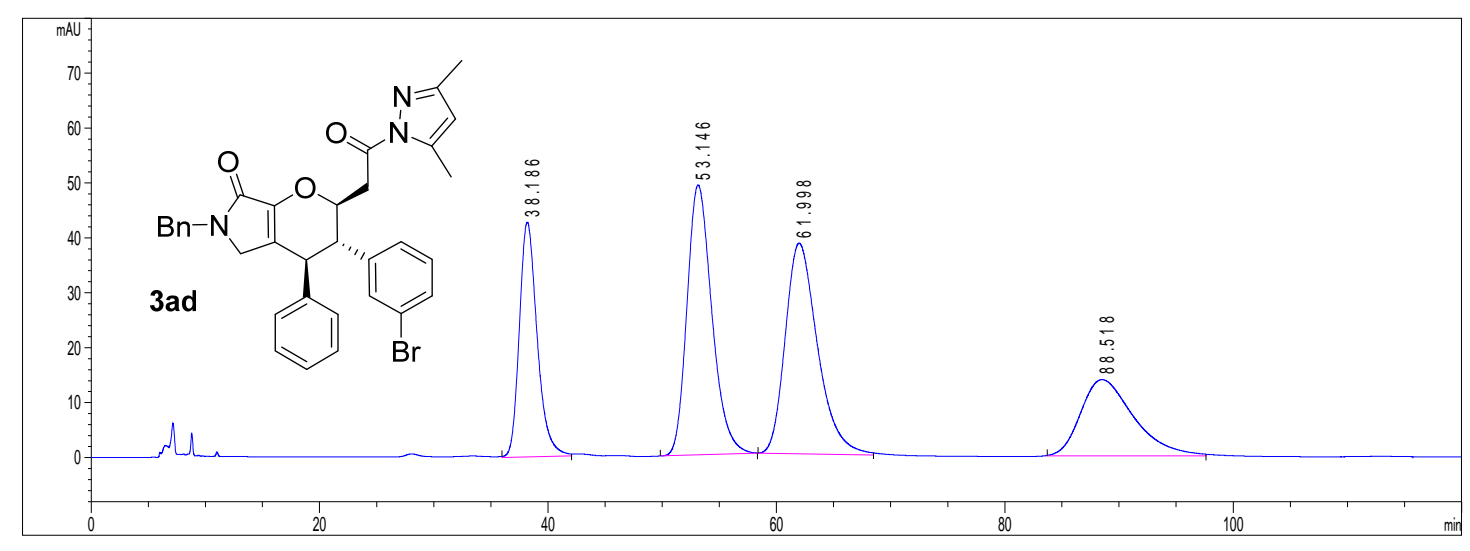

\begin{tabular}{|l|l|l|l|}
\hline & Retention & Area & Area\% \\
\hline 1 & 38.186 & 4641.11816 & 19.2733 \\
\hline 2 & 53.146 & 7442.02344 & 30.9048 \\
\hline 3 & 61.998 & 7526.66211 & 31.2562 \\
\hline 4 & 88.518 & 4470.70898 & 18.5657 \\
\hline
\end{tabular}

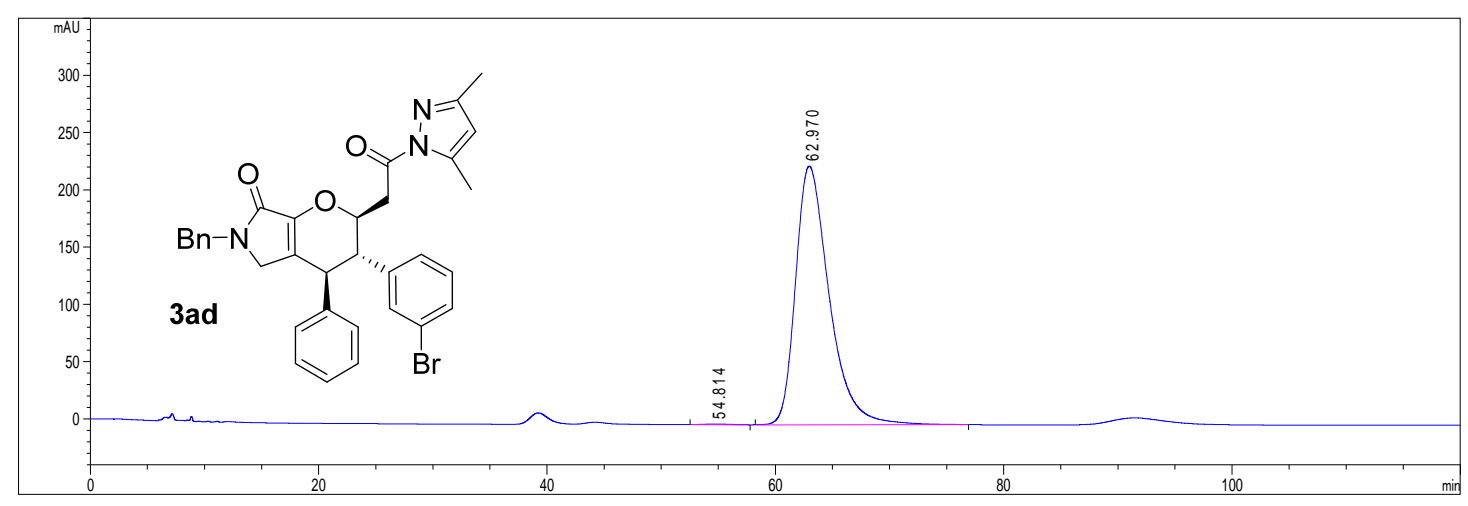

\begin{tabular}{|l|l|l|l|}
\hline & Retention & Area & Area\% \\
\hline 1 & 54.814 & 94.14662 & 0.2031 \\
\hline 2 & 62.970 & $4.62518 \mathrm{e} 4$ & 99.7969 \\
\hline
\end{tabular}




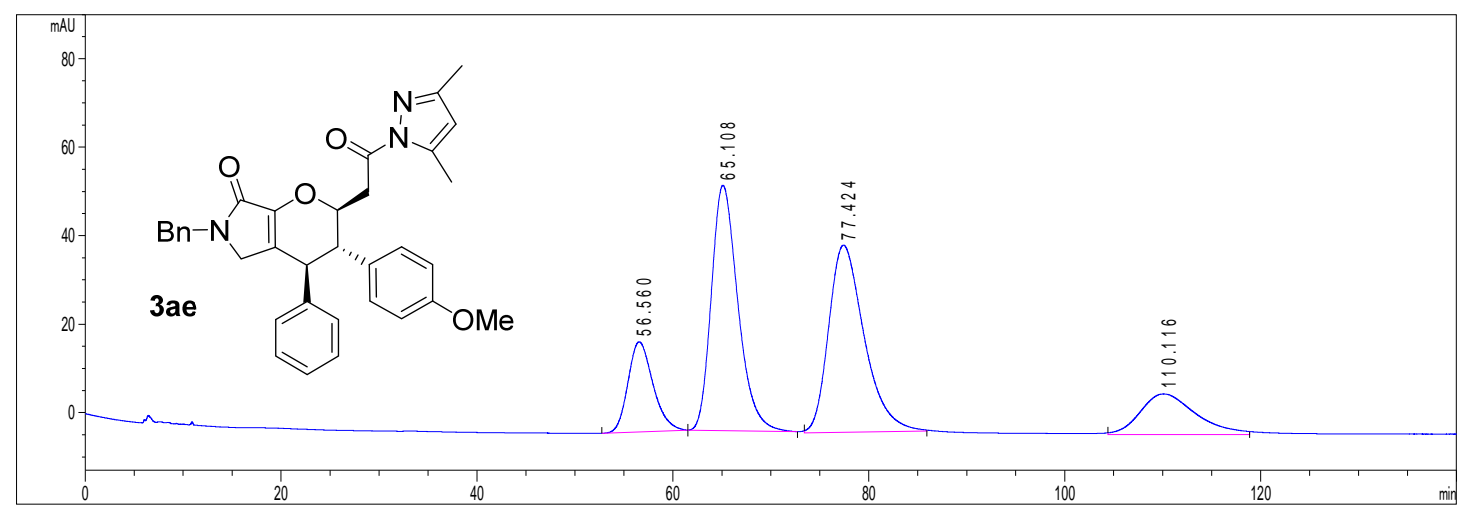

\begin{tabular}{|l|l|l|l|}
\hline & Retention & Area & Area $\%$ \\
\hline 1 & 56.560 & 3599.75879 & 12.6896 \\
\hline 2 & 65.108 & $1.04571 \mathrm{e} 4$ & 36.8627 \\
\hline 3 & 77.424 & $1.06805 \mathrm{e} 4$ & 37.6501 \\
\hline 4 & 110.116 & 3630.37109 & 12.7976 \\
\hline
\end{tabular}

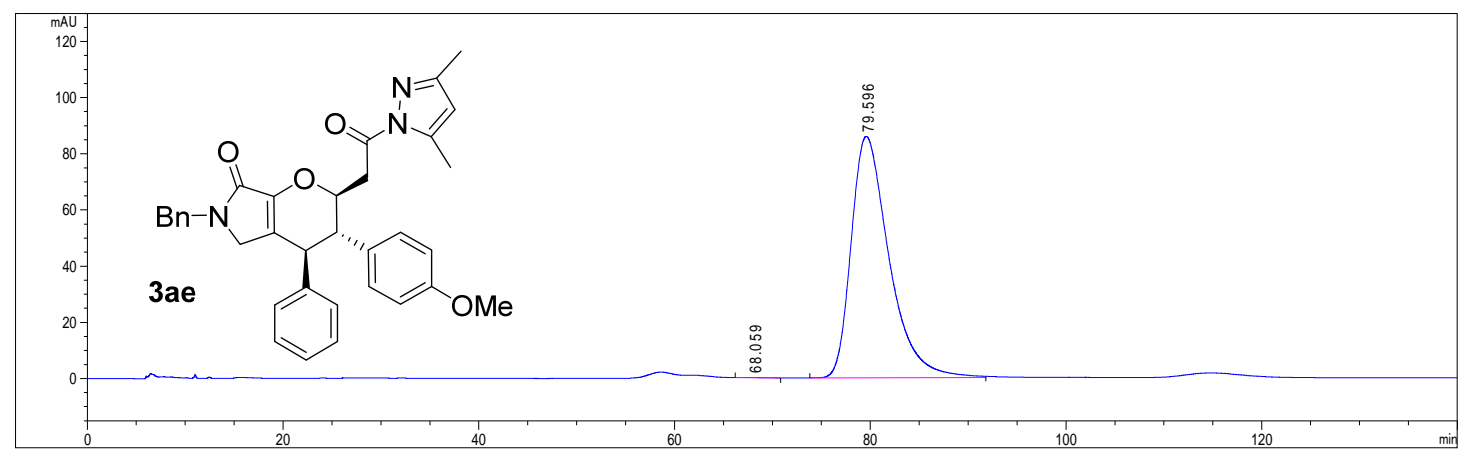

\begin{tabular}{|l|l|l|l|}
\hline & Retention & Area & Area\% \\
\hline 1 & 68.059 & 9.02058 & 0.0390 \\
\hline 2 & 79.596 & $2.31284 \mathrm{e} 4$ & 99.9610 \\
\hline
\end{tabular}




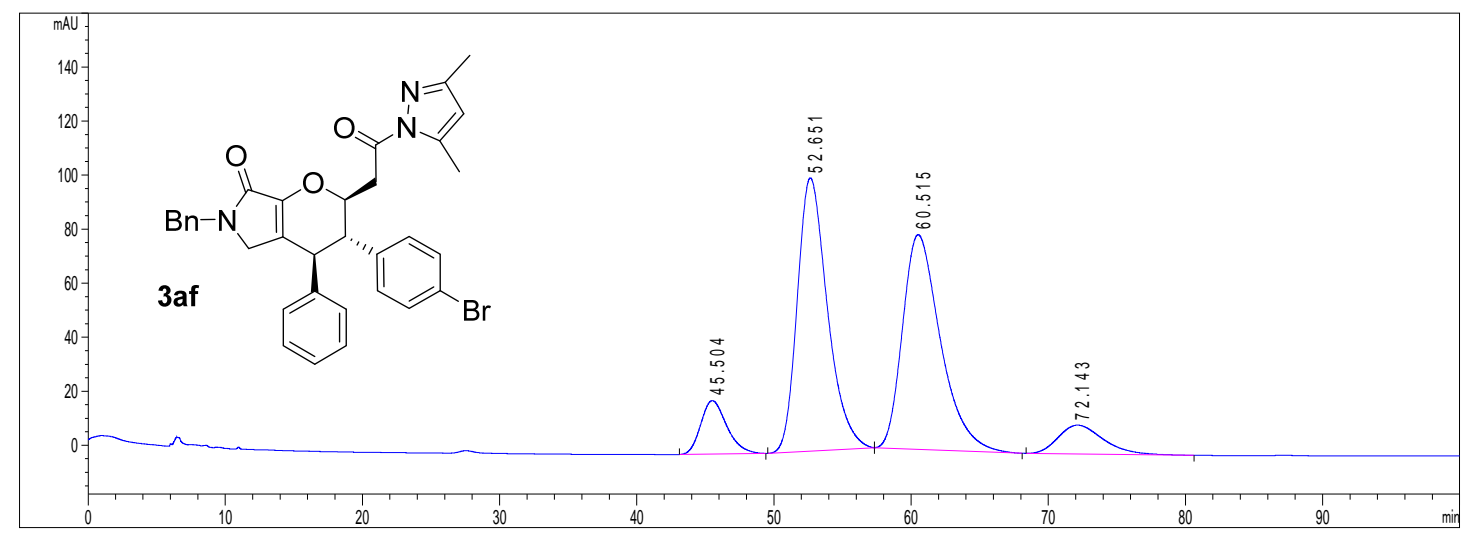

\begin{tabular}{|l|l|l|l|}
\hline & Retention & Area & Area\% \\
\hline 1 & 45.504 & 2612.39233 & 7.3133 \\
\hline 2 & 52.651 & $1.54132 \mathrm{e} 4$ & 43.1488 \\
\hline 3 & 60.515 & $1.52602 \mathrm{e} 4$ & 42.7205 \\
\hline 4 & 72.143 & 2435.24780 & 6.8174 \\
\hline
\end{tabular}

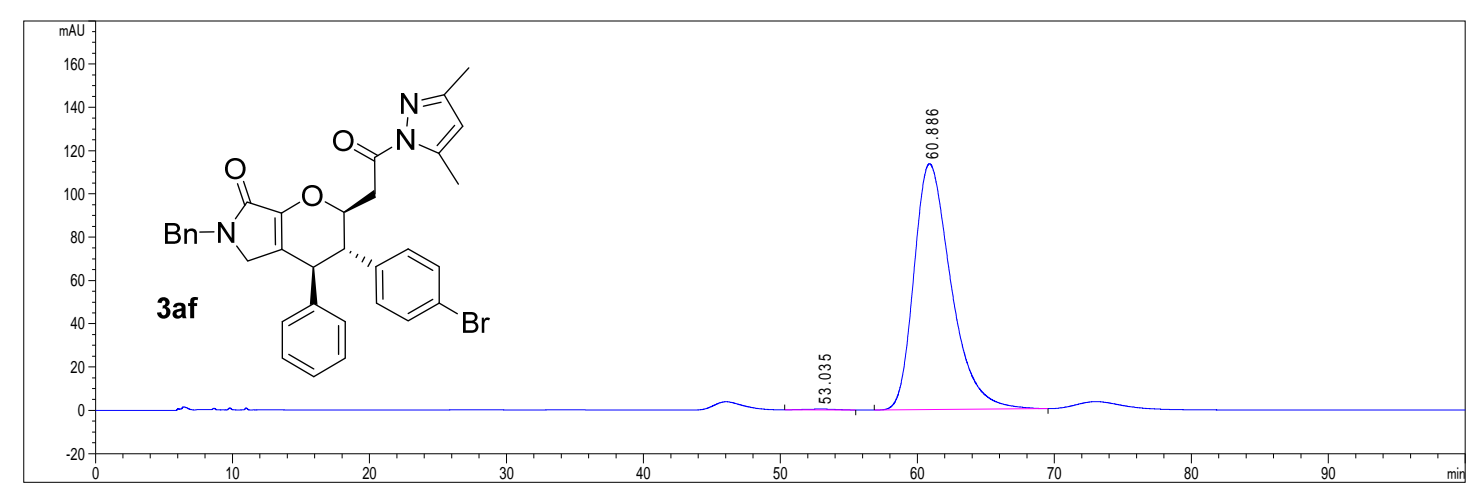

\begin{tabular}{|l|l|l|l|}
\hline & Retention & Area & Area\% \\
\hline 1 & 53.035 & 71.76995 & 0.3339 \\
\hline 2 & 60.886 & $2.14208 \mathrm{e} 4$ & 99.6661 \\
\hline
\end{tabular}




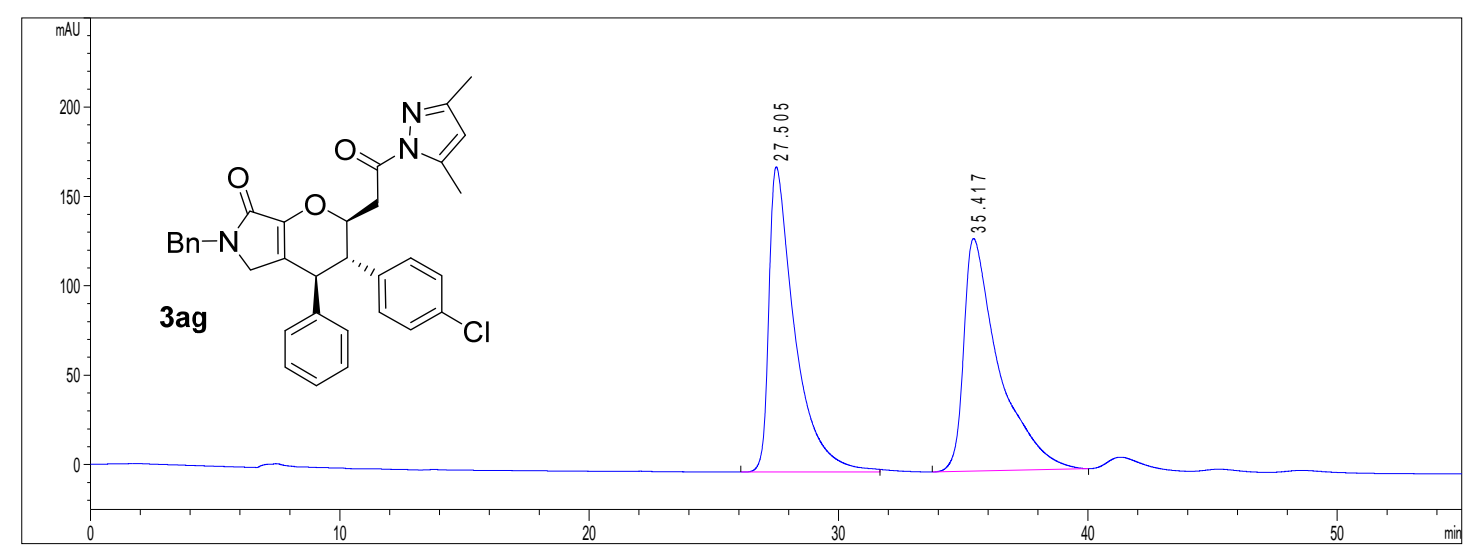

\begin{tabular}{|l|l|l|l|}
\hline & Retention & Area & Area\% \\
\hline 1 & 27.505 & $1.23802 \mathrm{e} 4$ & 49.2273 \\
\hline 2 & 35.417 & $1.27689 \mathrm{e} 4$ & 50.7727 \\
\hline
\end{tabular}

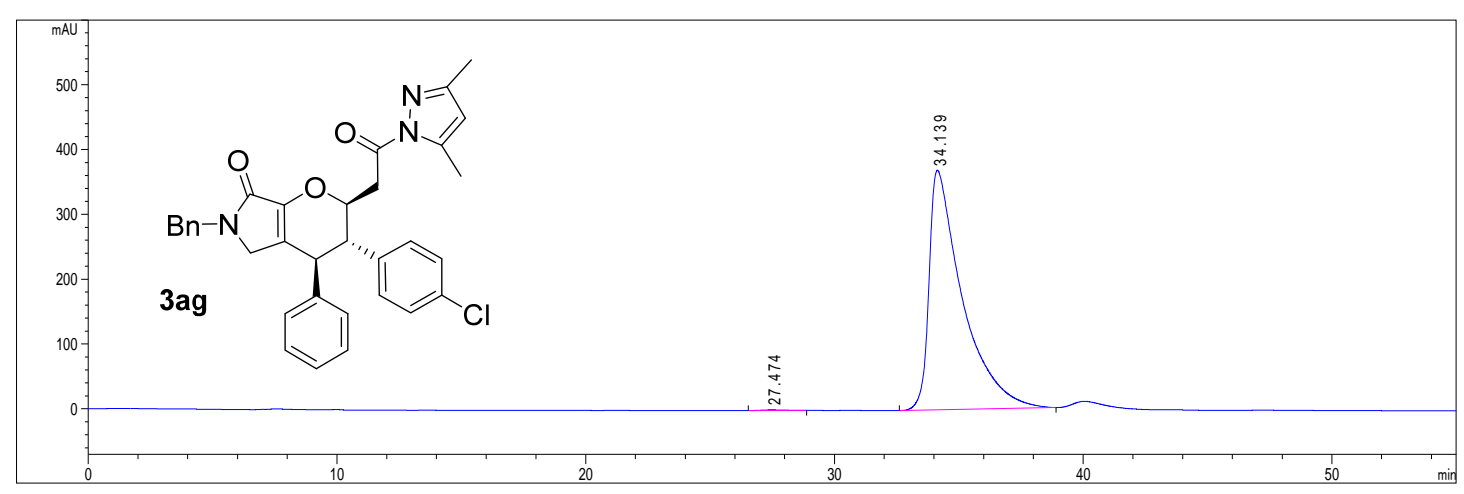

\begin{tabular}{|l|l|l|l|}
\hline & Retention & Area & Area\% \\
\hline 1 & 27.474 & 64.50605 & 0.1875 \\
\hline 2 & 34.139 & $3.43423 \mathrm{e} 4$ & 99.8125 \\
\hline
\end{tabular}




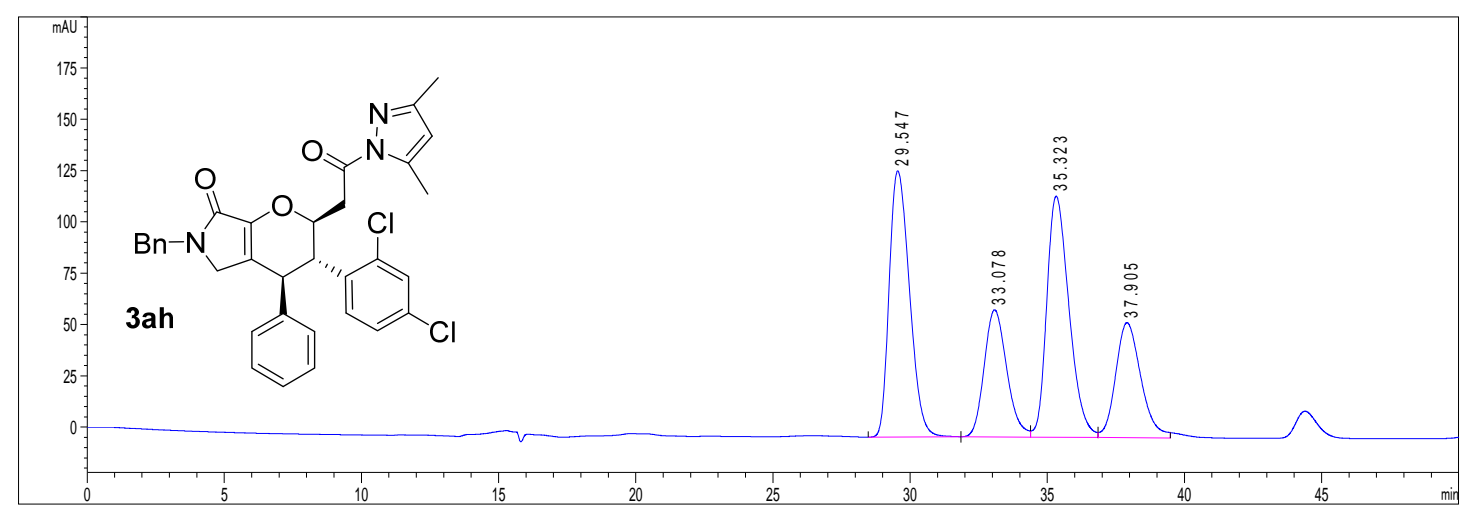

\begin{tabular}{|l|l|l|l|}
\hline & Retention & Area & Area $\%$ \\
\hline 1 & 29.547 & 6654.19092 & 32.5588 \\
\hline 2 & 33.078 & 3549.45337 & 17.3674 \\
\hline 3 & 35.323 & 6650.88965 & 32.5427 \\
\hline 4 & 37.905 & 3582.90918 & 17.5311 \\
\hline
\end{tabular}

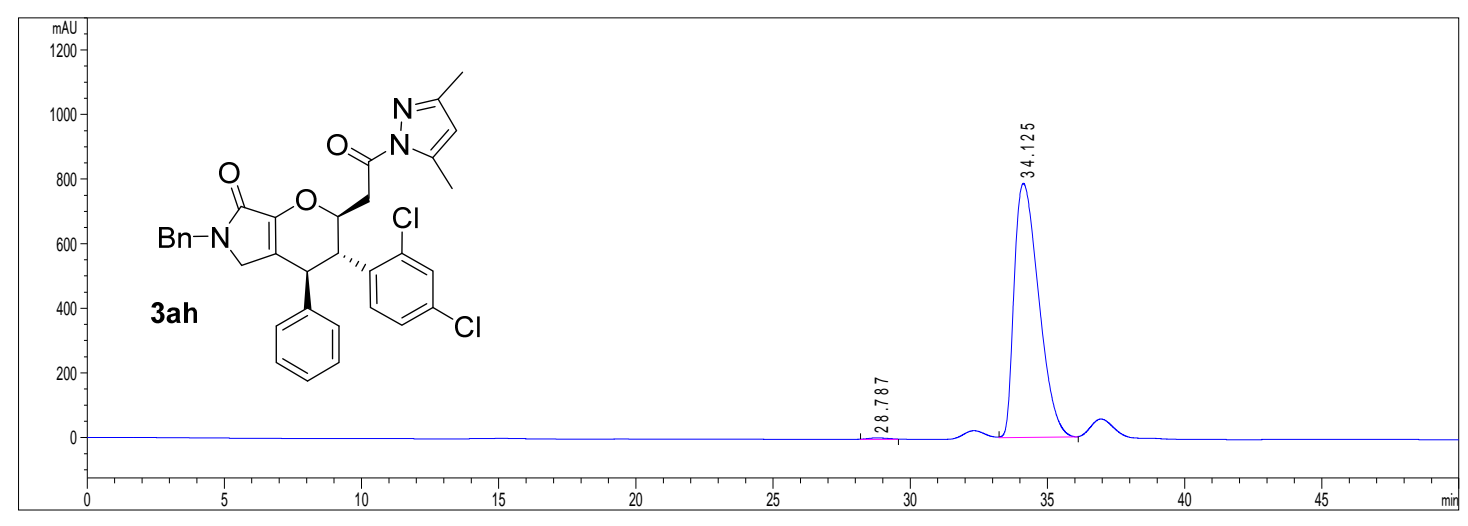

\begin{tabular}{|l|l|l|l|}
\hline & Retention & Area & Area\% \\
\hline 1 & 28.787 & 281.08179 & 0.5718 \\
\hline 2 & 34.125 & $4.88751 \mathrm{e} 4$ & 99.4282 \\
\hline
\end{tabular}




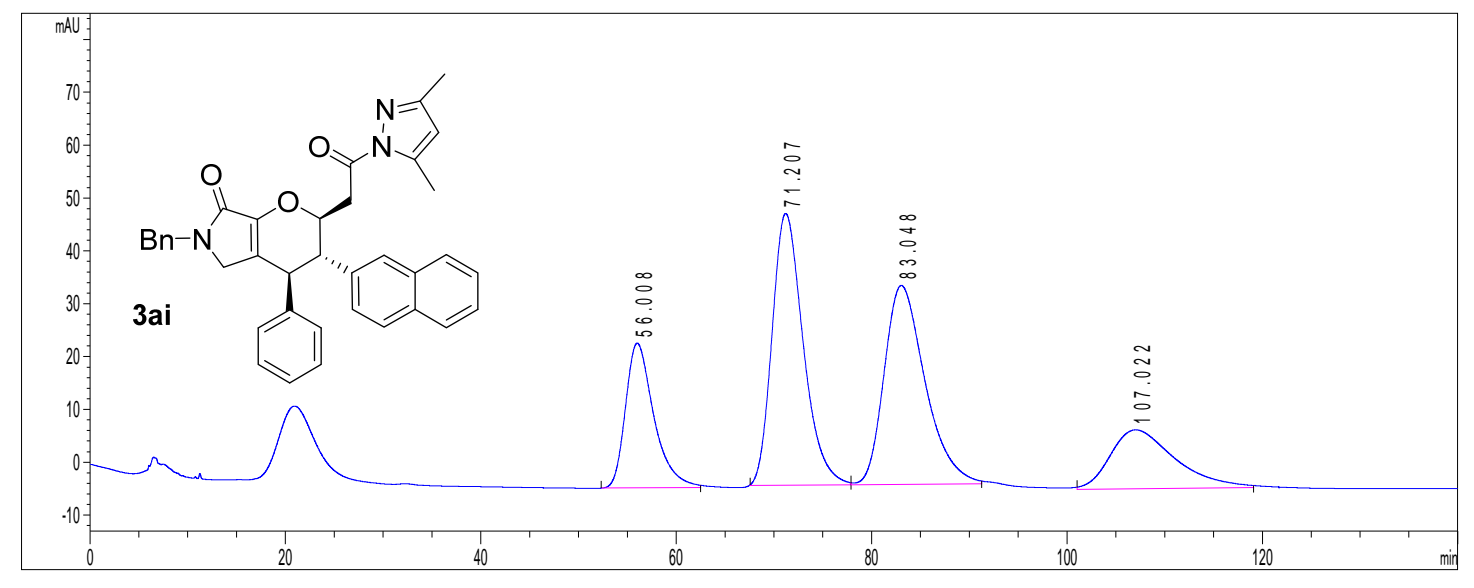

\begin{tabular}{|l|l|l|l|}
\hline & Retention & Area & Area\% \\
\hline 1 & 56.008 & 5540.55664 & 16.7804 \\
\hline 2 & 71.207 & $1.13874 \mathrm{e} 4$ & 34.4885 \\
\hline 3 & 83.048 & $1.09560 \mathrm{e} 4$ & 33.1820 \\
\hline 4 & 107.022 & 5134.00830 & 15.5491 \\
\hline
\end{tabular}

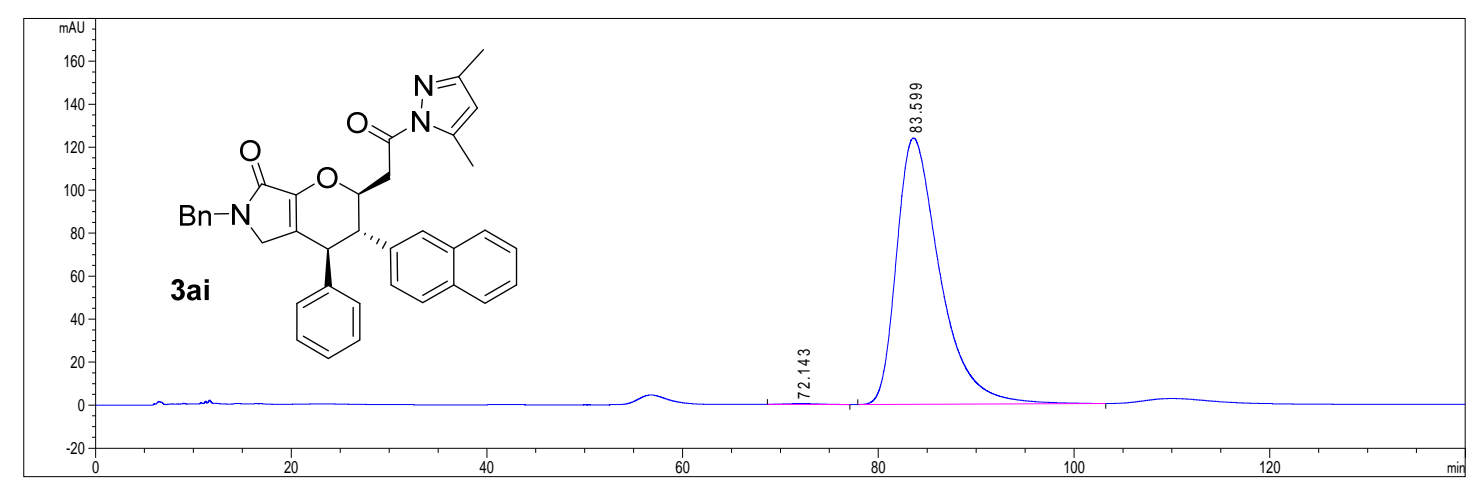

\begin{tabular}{|l|l|l|l|}
\hline & Retention & Area & Area $\%$ \\
\hline 1 & 72.143 & 104.57434 & 0.2731 \\
\hline 2 & 83.599 & $3.81859 \mathrm{e} 4$ & 99.7269 \\
\hline
\end{tabular}




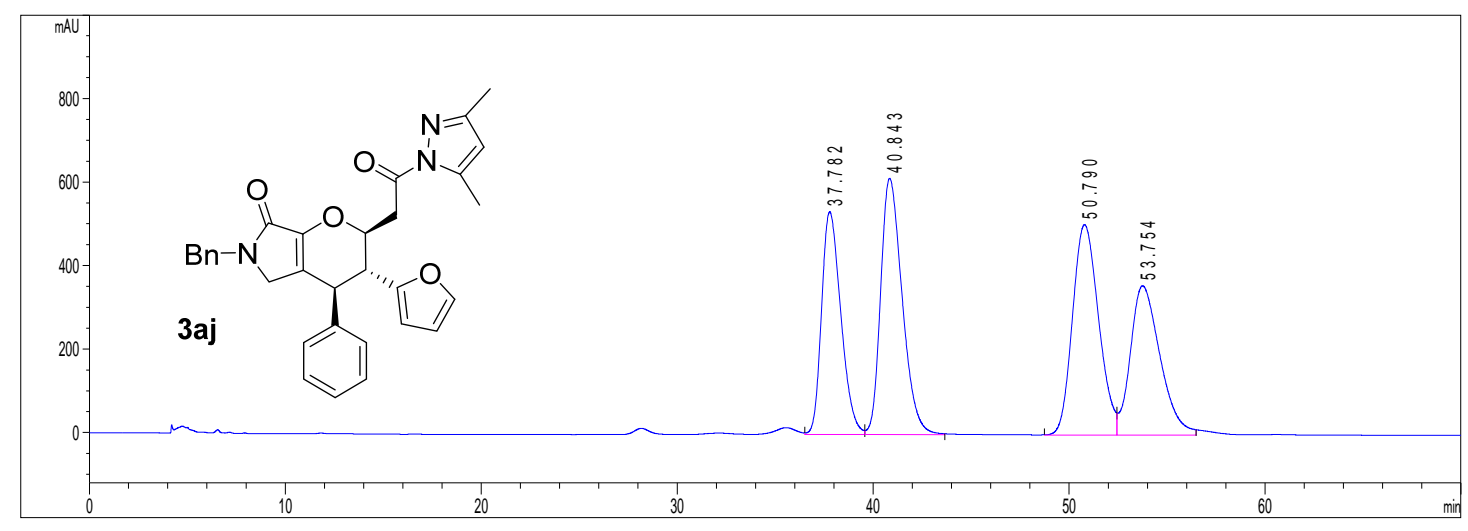

\begin{tabular}{|l|l|l|l|}
\hline & Retention & Area & Area\% \\
\hline 1 & 37.782 & $3.65101 \mathrm{e} 4$ & 21.7884 \\
\hline 2 & 40.843 & $4.69007 \mathrm{e} 4$ & 27.9894 \\
\hline 3 & 50.790 & $4.65931 \mathrm{e} 4$ & 27.8058 \\
\hline 4 & 53.754 & $3.75624 \mathrm{e} 4$ & 22.4164 \\
\hline
\end{tabular}

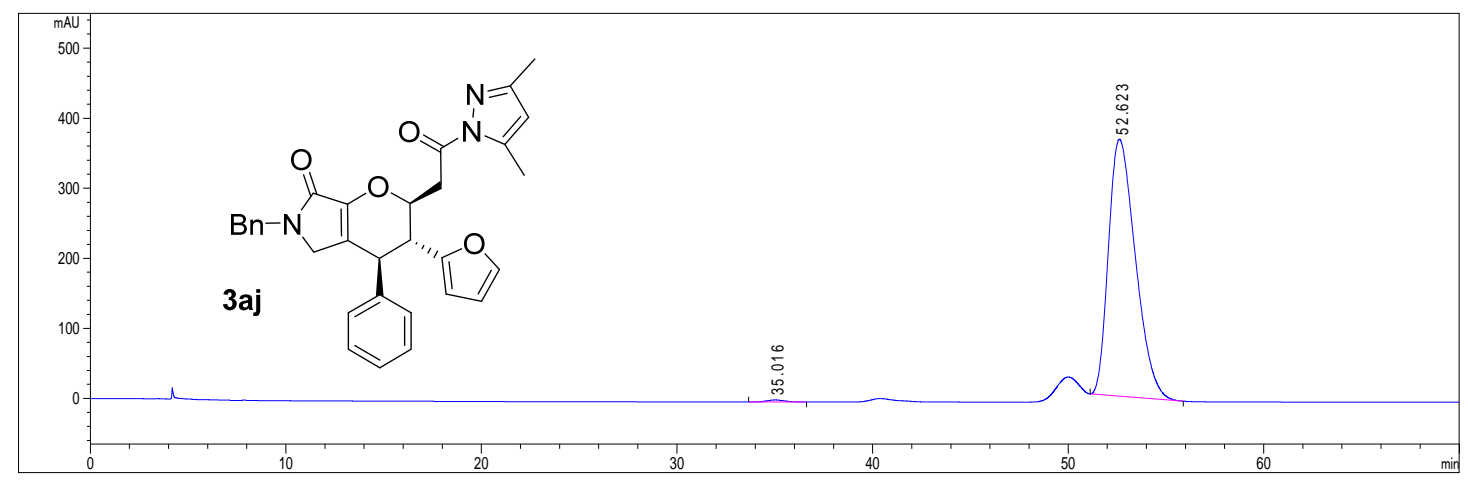

\begin{tabular}{|l|l|l|l|}
\hline & Retention & Area & Area\% \\
\hline 1 & 35.016 & 176.49234 & 0.4994 \\
\hline 2 & 52.623 & $3.51669 \mathrm{e} 4$ & 99.5006 \\
\hline
\end{tabular}




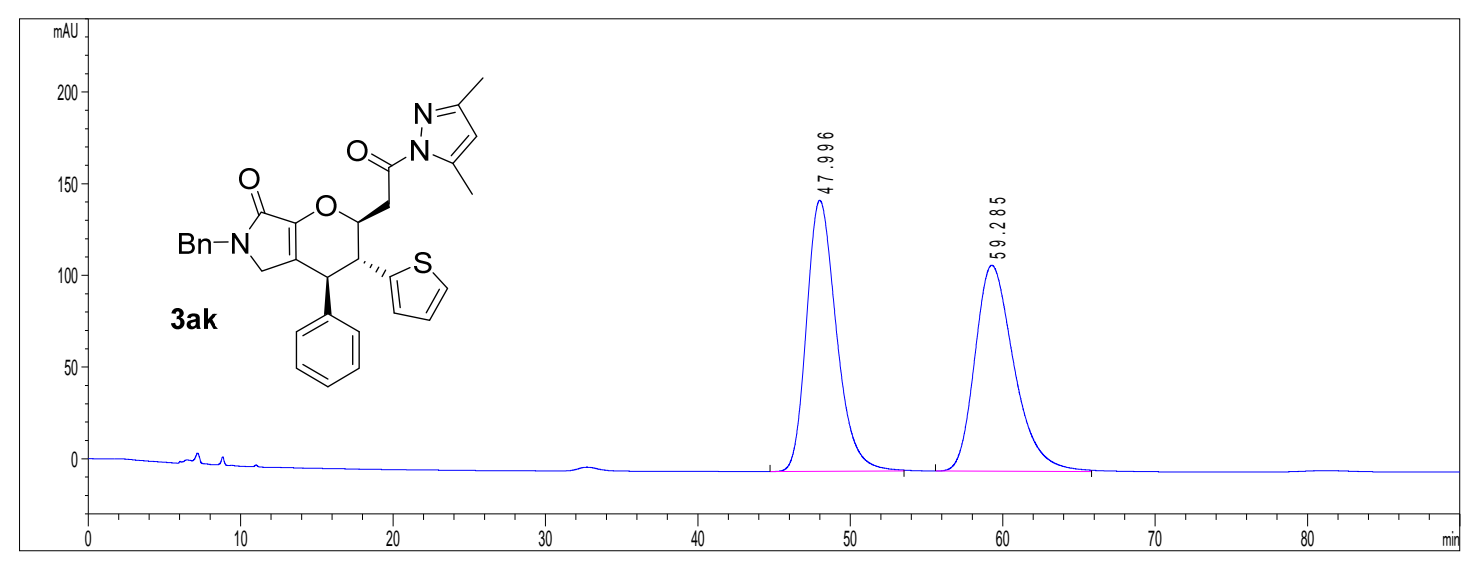

\begin{tabular}{|l|l|l|l|}
\hline & Retention & Area & Area\% \\
\hline 1 & 47.996 & $2.01609 \mathrm{e} 4$ & 50.1370 \\
\hline 2 & 59.285 & $2.00508 \mathrm{e} 4$ & 49.8630 \\
\hline
\end{tabular}

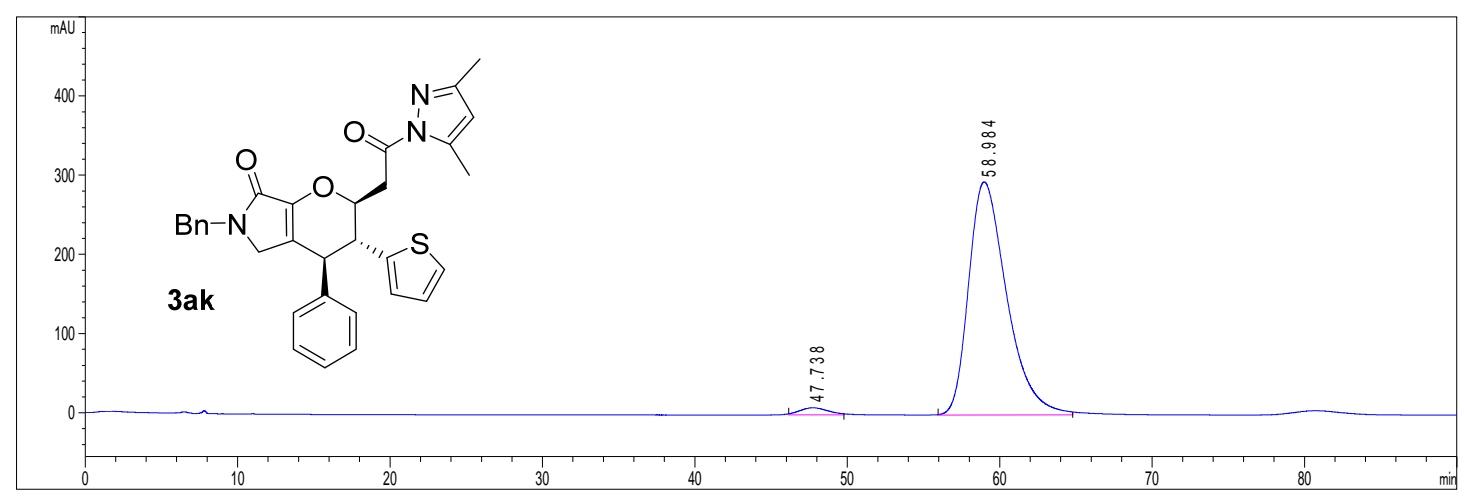

\begin{tabular}{|l|l|l|l|}
\hline & Retention & Area & Area $\%$ \\
\hline 1 & 47.738 & 1181.82312 & 2.2555 \\
\hline 2 & 58.984 & $5.12163 \mathrm{e} 4$ & 97.7445 \\
\hline
\end{tabular}




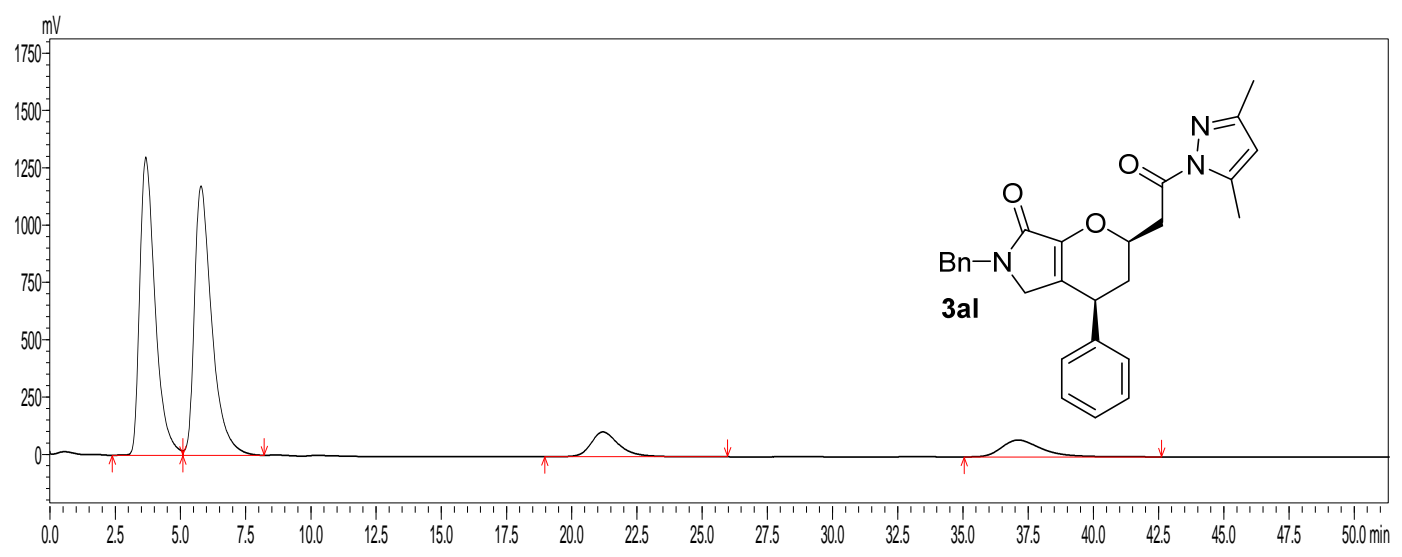

\begin{tabular}{|l|l|l|l|}
\hline & Retention & Area & Area\% \\
\hline 1 & 3.673 & 53315347 & 43.131 \\
\hline 2 & 5.787 & 54453027 & 44.051 \\
\hline 3 & 21.195 & 8117473 & 6.567 \\
\hline 4 & 37.112 & 7727013 & 6.251 \\
\hline
\end{tabular}

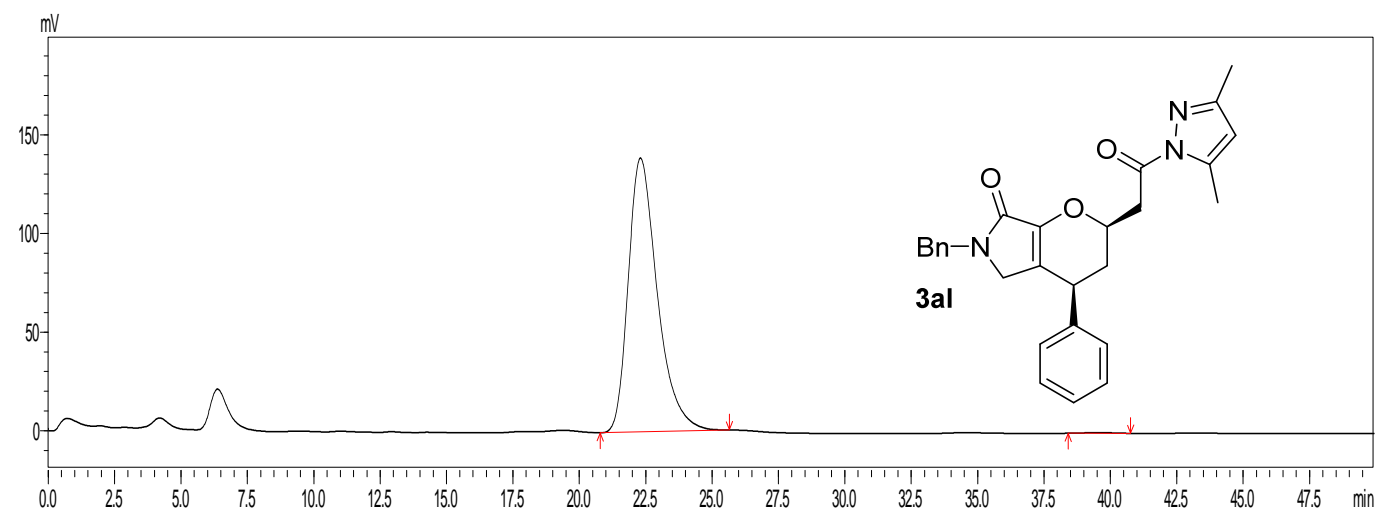

\begin{tabular}{|l|l|l|l|}
\hline & Retention & Area & Area $\%$ \\
\hline 1 & 22.303 & 10581922 & 99.760 \\
\hline 2 & 39.566 & 25429 & 0.240 \\
\hline
\end{tabular}




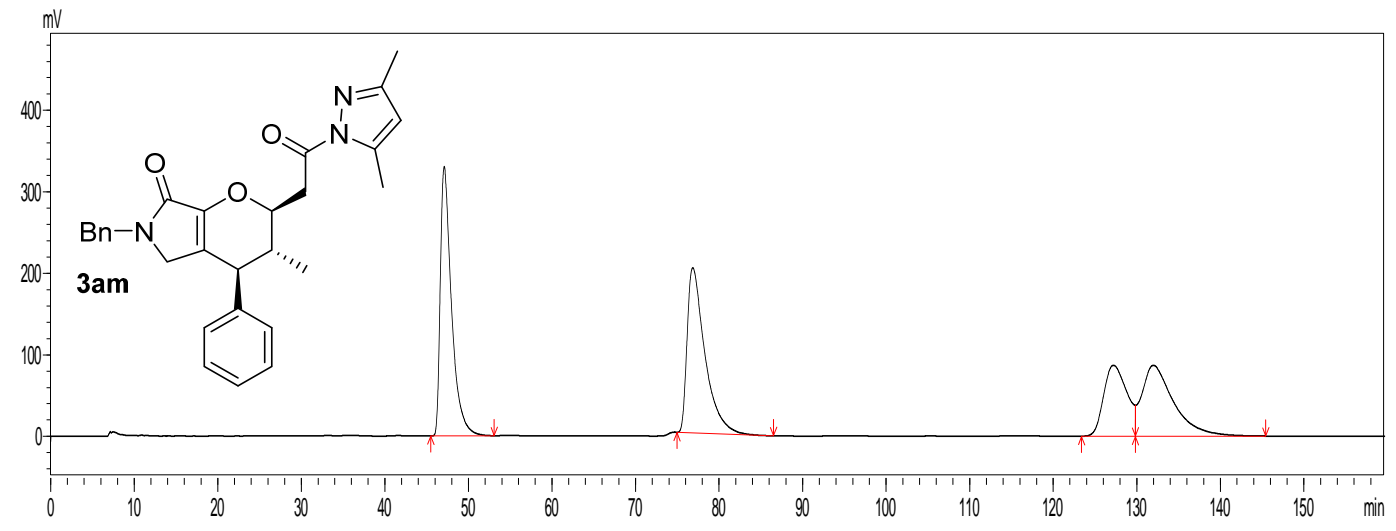

\begin{tabular}{|l|l|l|l|}
\hline & Retention & Area & Area\% \\
\hline 1 & 47.099 & 30763196 & 30.176 \\
\hline 2 & 76.856 & 31545776 & 30.943 \\
\hline 3 & 127.206 & 16811248 & 16.490 \\
\hline 4 & 131.987 & 22826711 & 22.391 \\
\hline
\end{tabular}

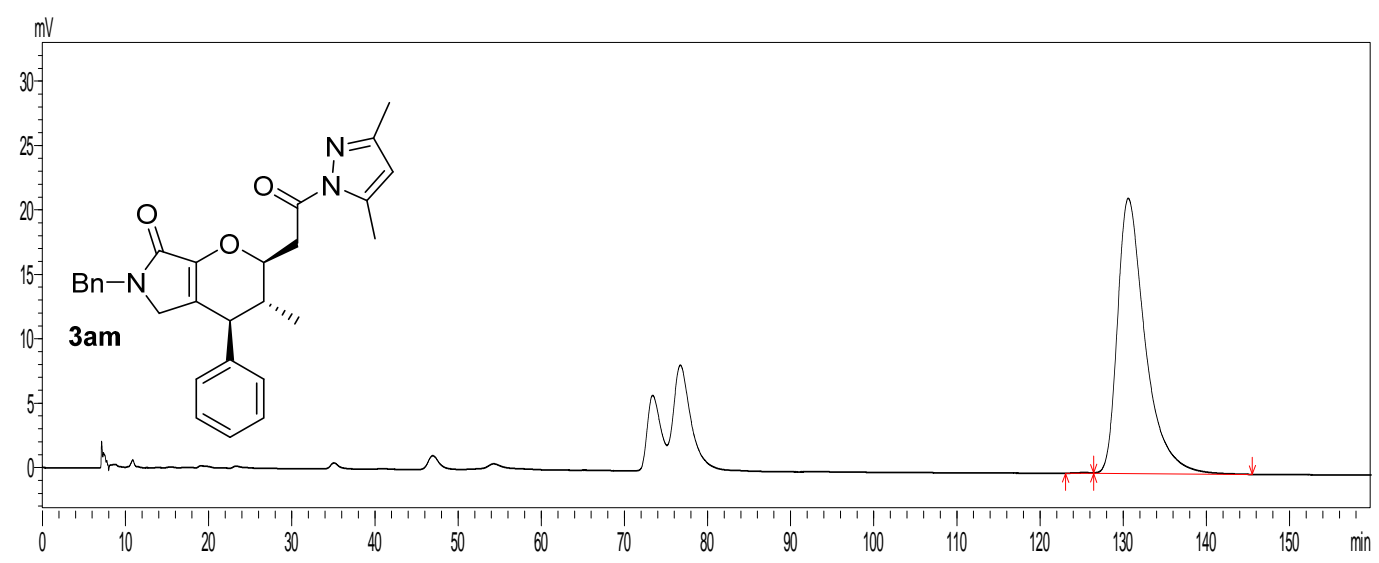

\begin{tabular}{|l|l|l|l|}
\hline & Retention & Area & Area $\%$ \\
\hline 1 & 125.437 & 8257 & 0.166 \\
\hline 2 & 130.603 & 4958269 & 99.834 \\
\hline
\end{tabular}




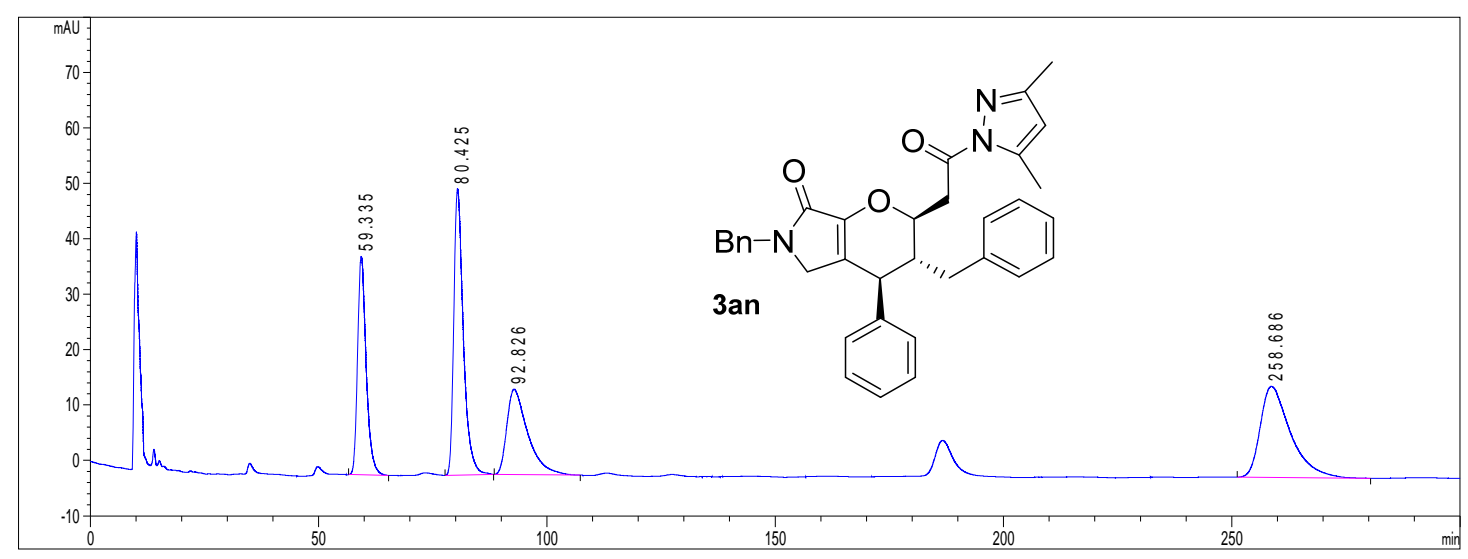

\begin{tabular}{|l|l|l|l|}
\hline & Retention & Area & Area\% \\
\hline 1 & 59.335 & 5145.68408 & 20.4205 \\
\hline 2 & 80.425 & 7516.56396 & 29.8293 \\
\hline 3 & 92.826 & 4919.26270 & 19.5220 \\
\hline 4 & 258.686 & 7617.10791 & 30.2283 \\
\hline
\end{tabular}

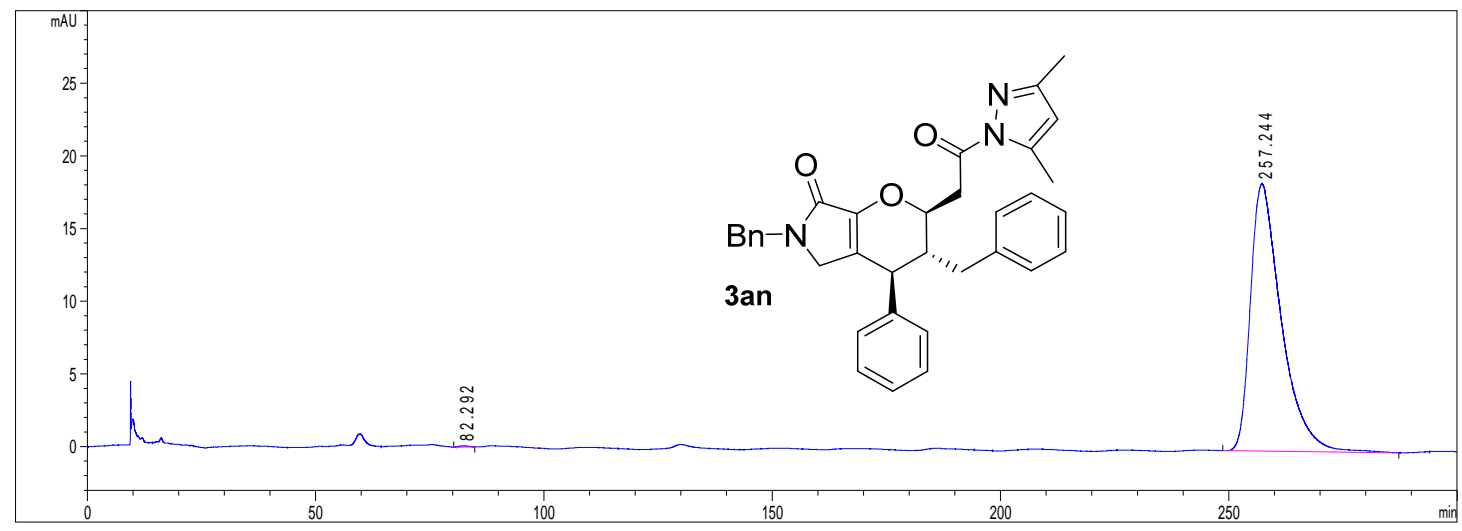

\begin{tabular}{|l|l|l|l|}
\hline & Retention & Area & Area $\%$ \\
\hline 1 & 82.292 & 12.80312 & 0.1476 \\
\hline 2 & 257.244 & 8659.46094 & 99.8524 \\
\hline
\end{tabular}




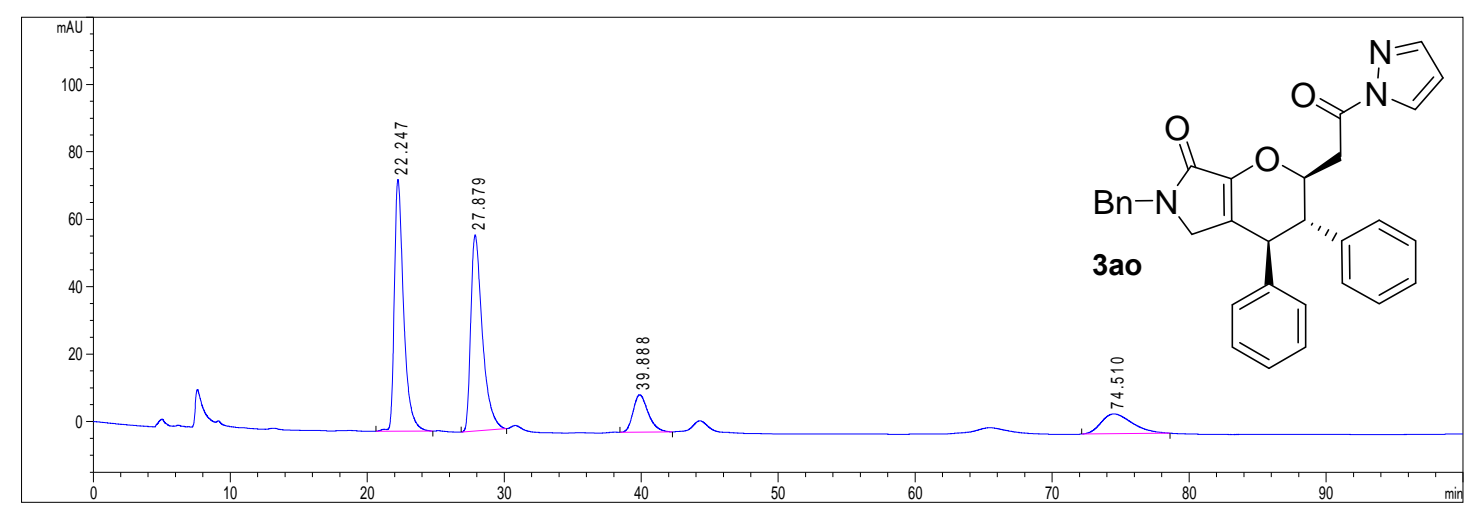

\begin{tabular}{|l|l|l|l|}
\hline & Retention & Area & Area\% \\
\hline 1 & 22.247 & 3514.24414 & 40.3996 \\
\hline 2 & 27.879 & 3418.52856 & 39.2993 \\
\hline 3 & 39.888 & 842.17352 & 9.6816 \\
\hline 4 & 74.510 & 923.76141 & 10.6195 \\
\hline
\end{tabular}

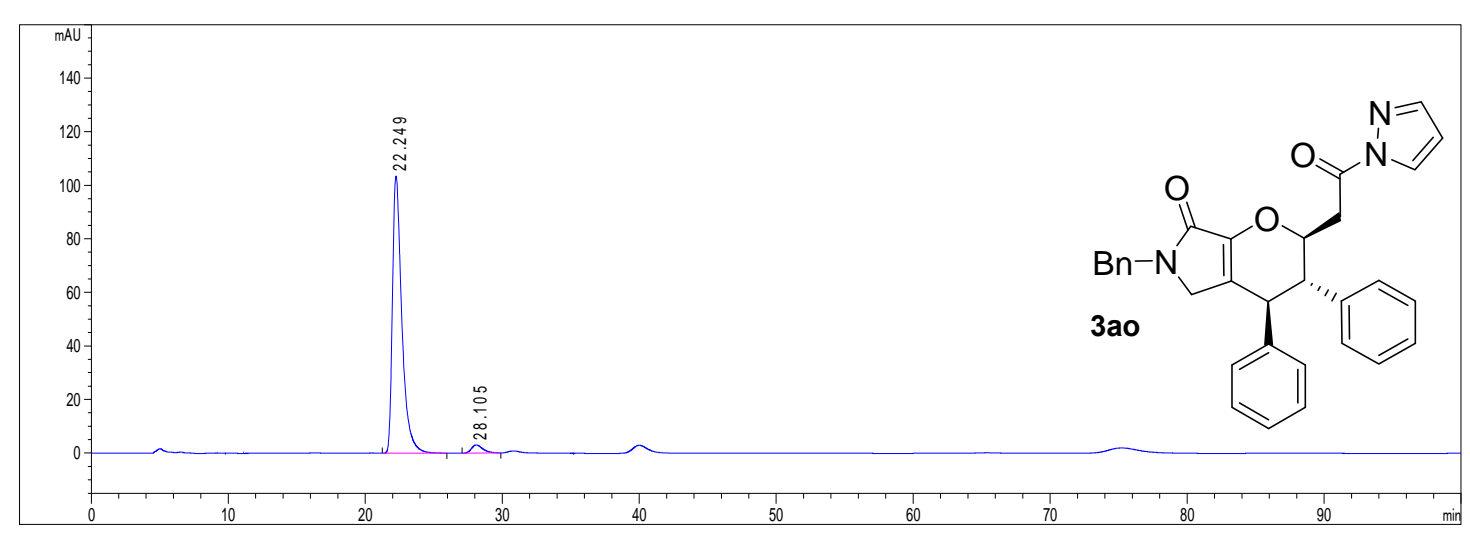

\begin{tabular}{|l|l|l|l|}
\hline & Retention & Area & Area\% \\
\hline 1 & 22.249 & 4932.13721 & 96.5477 \\
\hline 2 & 28.105 & 176.36075 & 3.4523 \\
\hline
\end{tabular}




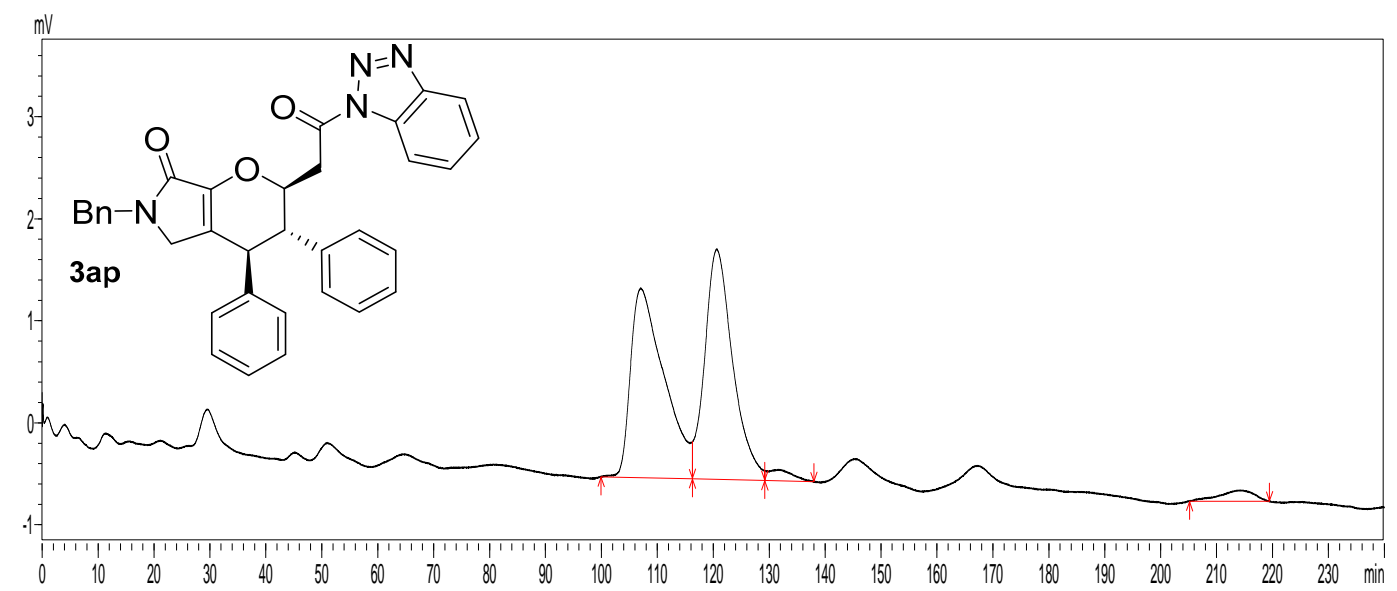

\begin{tabular}{|l|l|l|l|}
\hline & Retention & Area & Area\% \\
\hline 1 & 107.038 & 775694 & 47.306 \\
\hline 2 & 120.620 & 783813 & 47.801 \\
\hline 3 & 131.656 & 33687 & 2.054 \\
\hline 4 & 214.183 & 46550 & 2.839 \\
\hline
\end{tabular}

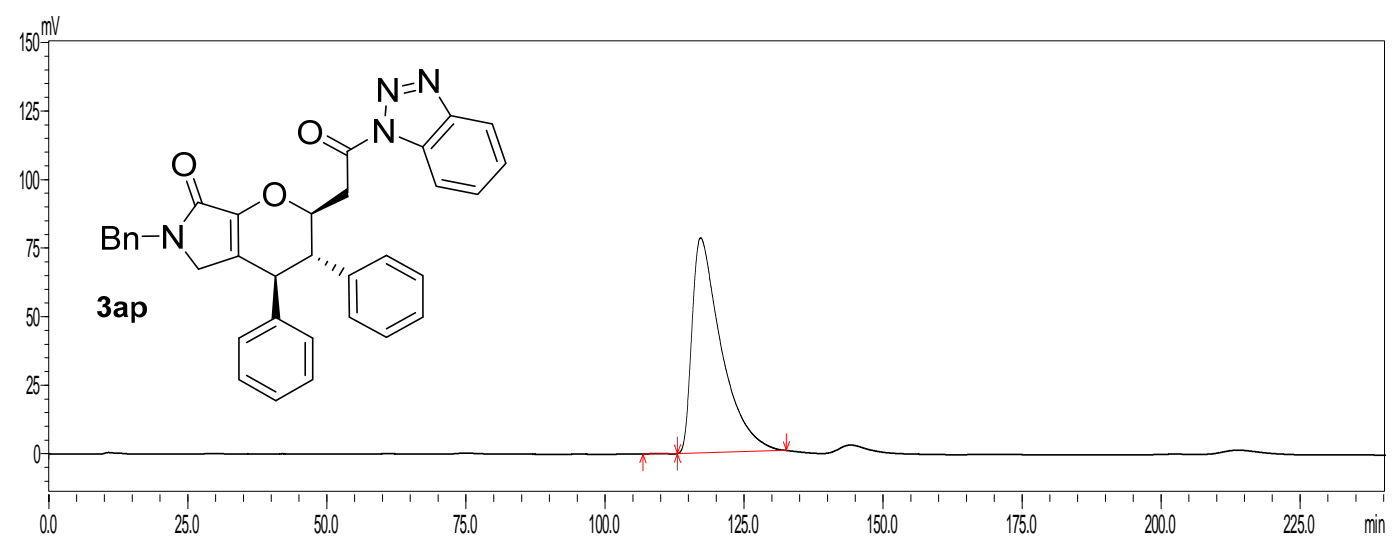

\begin{tabular}{|l|l|l|l|}
\hline & Retention & Area & Area\% \\
\hline 1 & 109.668 & 14225 & 0.050 \\
\hline 2 & 117.194 & 28711987 & 99.950 \\
\hline
\end{tabular}



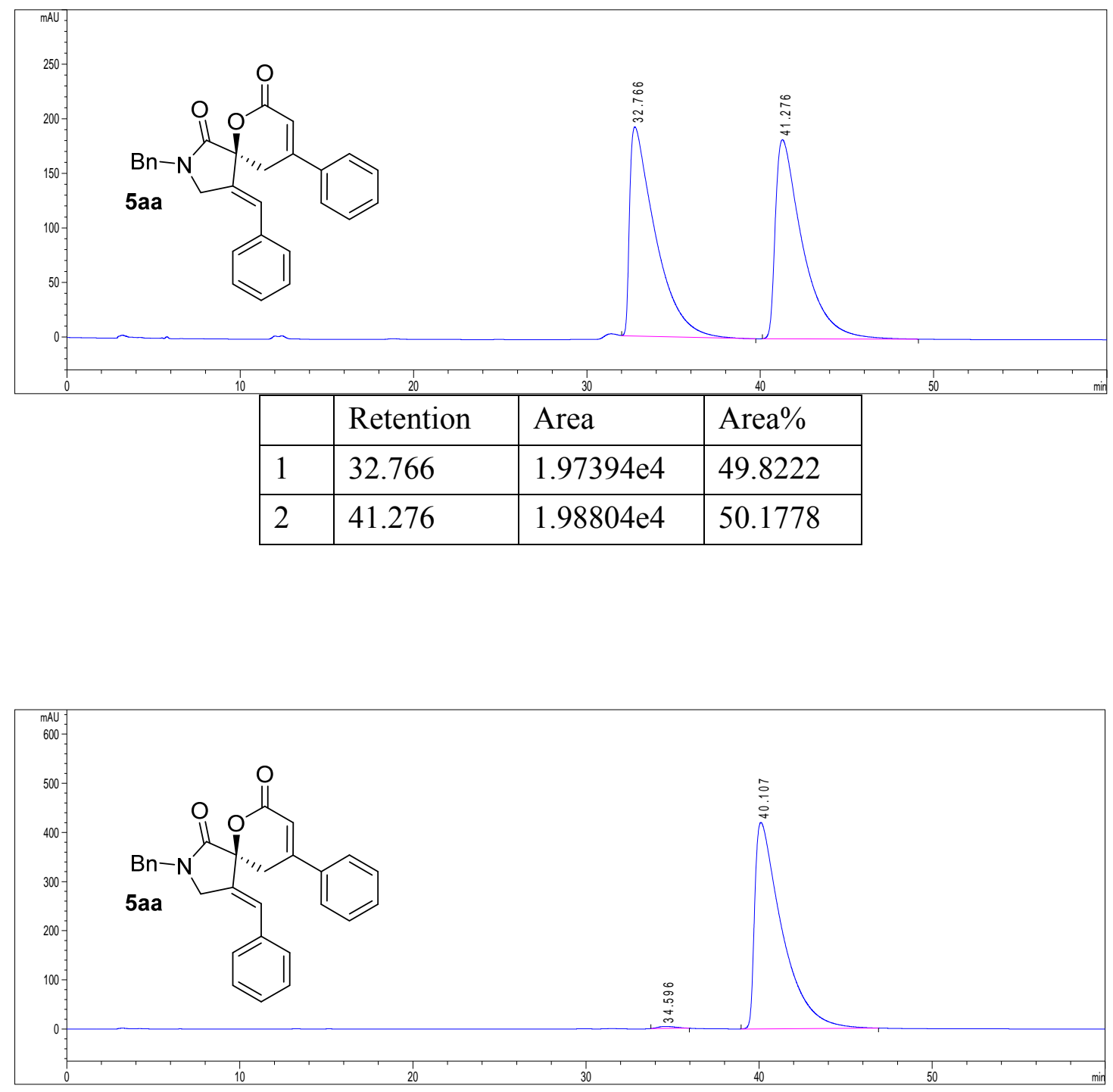

\begin{tabular}{|l|l|l|l|}
\hline & Retention & Area & Area\% \\
\hline 1 & 34.596 & 321.01535 & 0.6929 \\
\hline 2 & 40.107 & $4.60106 \mathrm{e} 4$ & 99.3071 \\
\hline
\end{tabular}




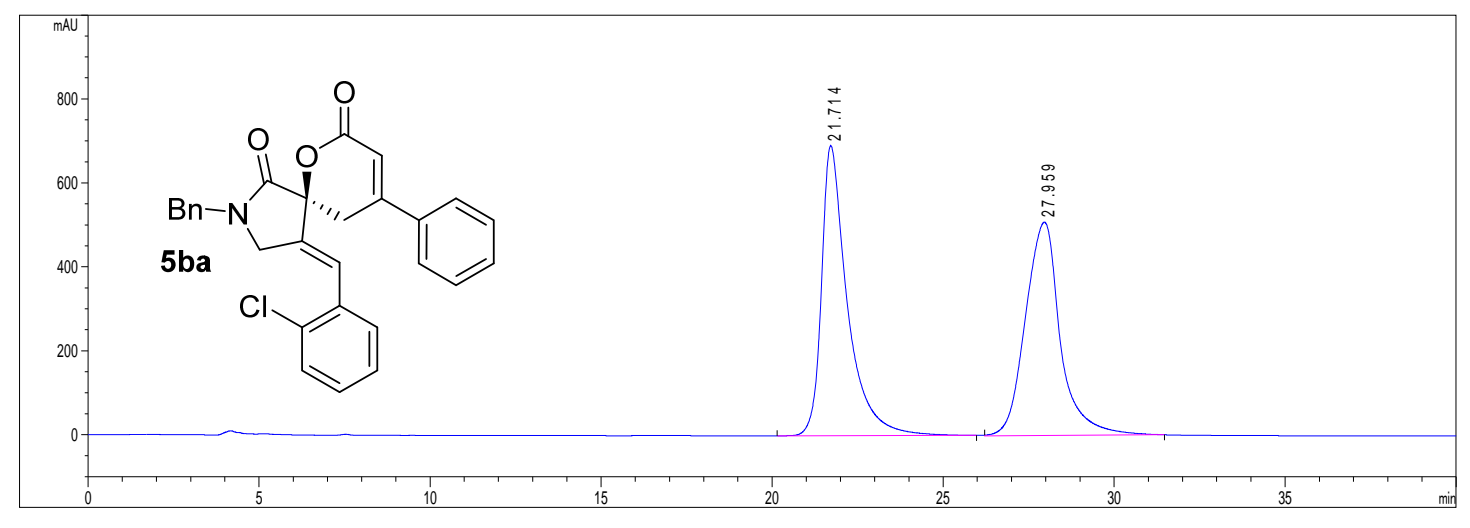

\begin{tabular}{|l|l|l|l|}
\hline & Retention & Area & Area $\%$ \\
\hline 1 & 21.714 & $3.64073 \mathrm{e} 4$ & 50.2970 \\
\hline 2 & 27.959 & $3.59773 \mathrm{e} 4$ & 49.7030 \\
\hline
\end{tabular}

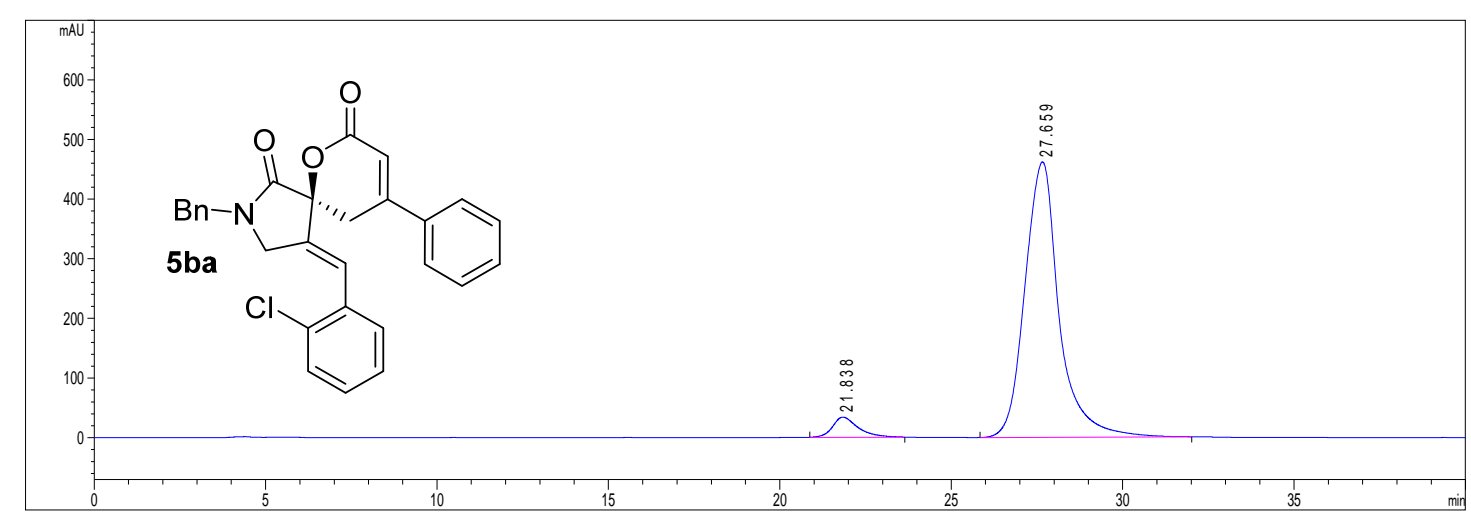

\begin{tabular}{|l|l|l|l|}
\hline & Retention & Area & Area\% \\
\hline 1 & 21.838 & 1755.63696 & 5.1981 \\
\hline 2 & 27.659 & $3.20191 \mathrm{e} 4$ & 94.8019 \\
\hline
\end{tabular}




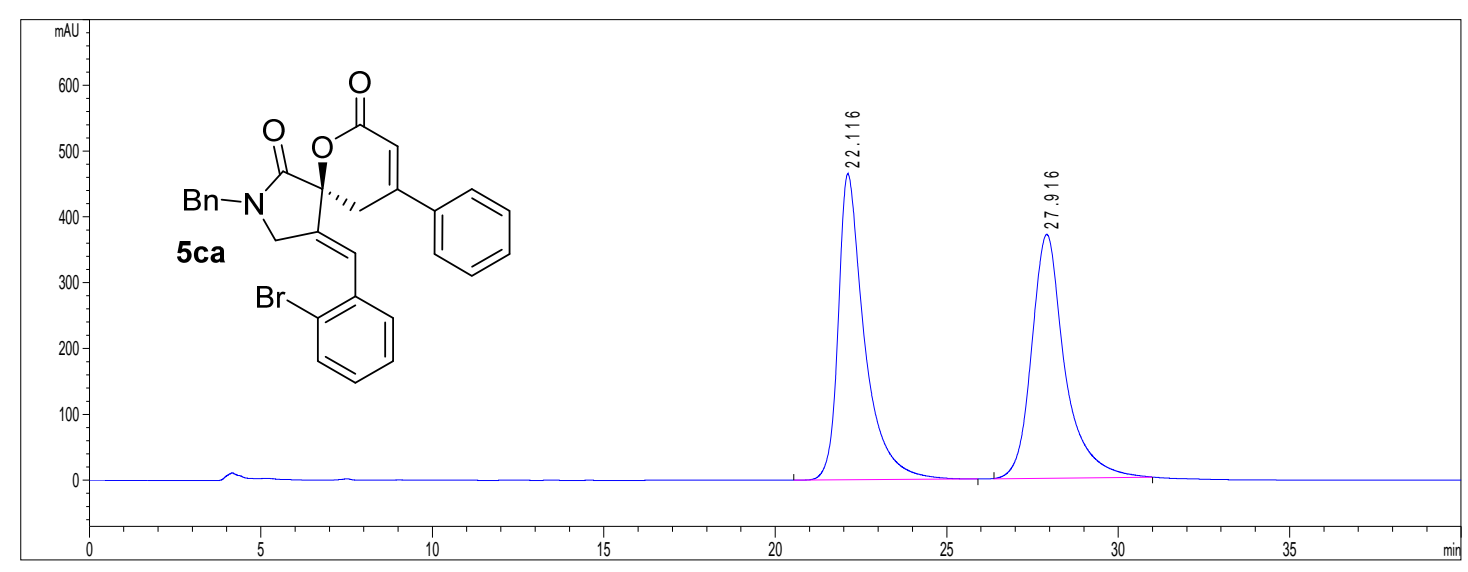

\begin{tabular}{|l|l|l|l|}
\hline & Retention & Area & Area\% \\
\hline 1 & 22.116 & $2.48100 \mathrm{e} 4$ & 49.7963 \\
\hline 2 & 27.916 & $2.50129 \mathrm{e} 4$ & 50.2037 \\
\hline
\end{tabular}

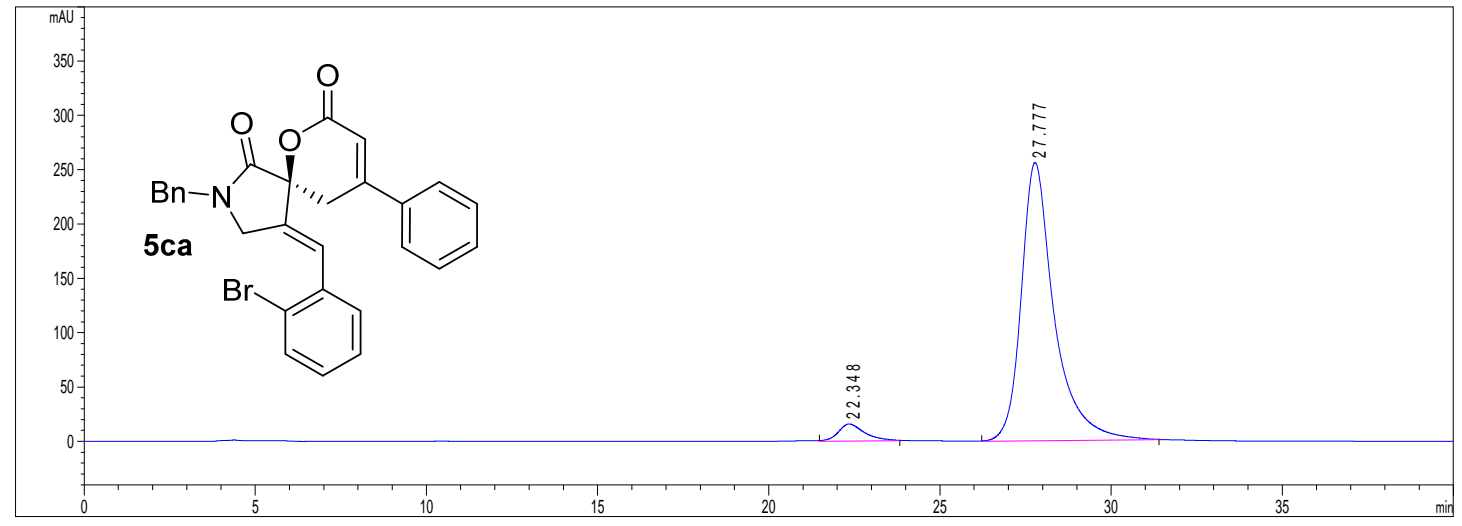

\begin{tabular}{|l|l|l|l|}
\hline & Retention & Area & Area\% \\
\hline 1 & 22.348 & 840.28406 & 4.6757 \\
\hline 2 & 27.777 & $1.71311 \mathrm{e} 4$ & 95.3243 \\
\hline
\end{tabular}




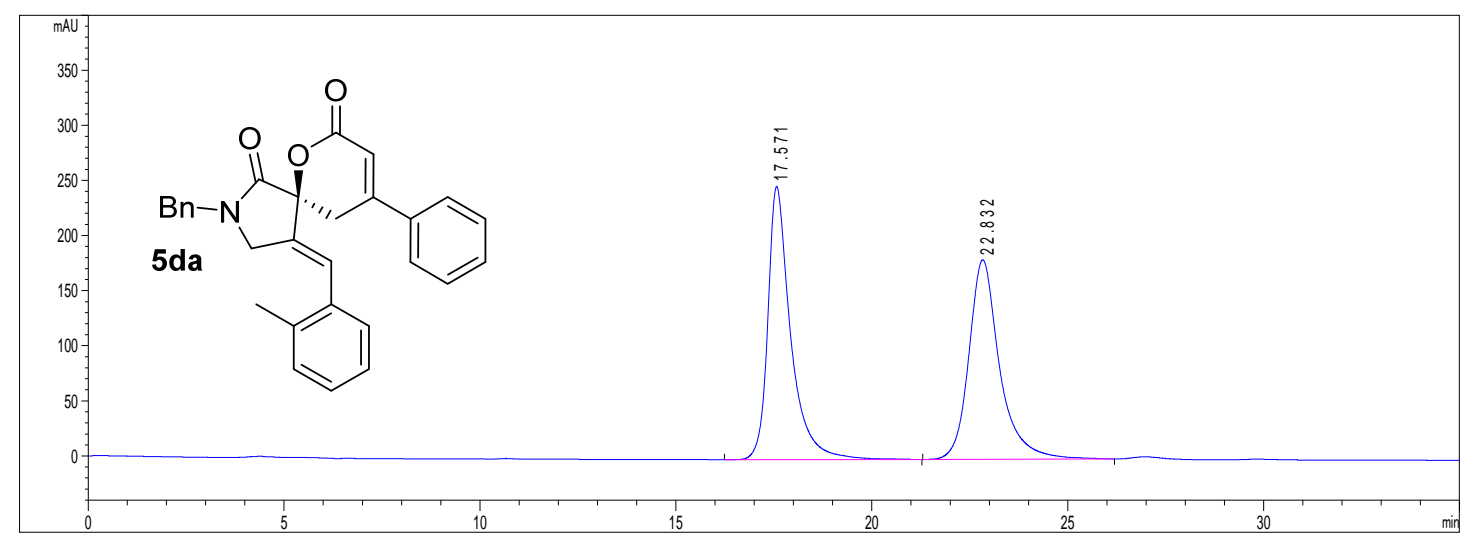

\begin{tabular}{|l|l|l|l|}
\hline & Retention & Area & Area\% \\
\hline 1 & 17.571 & $1.00453 \mathrm{e} 4$ & 50.4493 \\
\hline 2 & 22.832 & 9866.39355 & 49.5507 \\
\hline
\end{tabular}

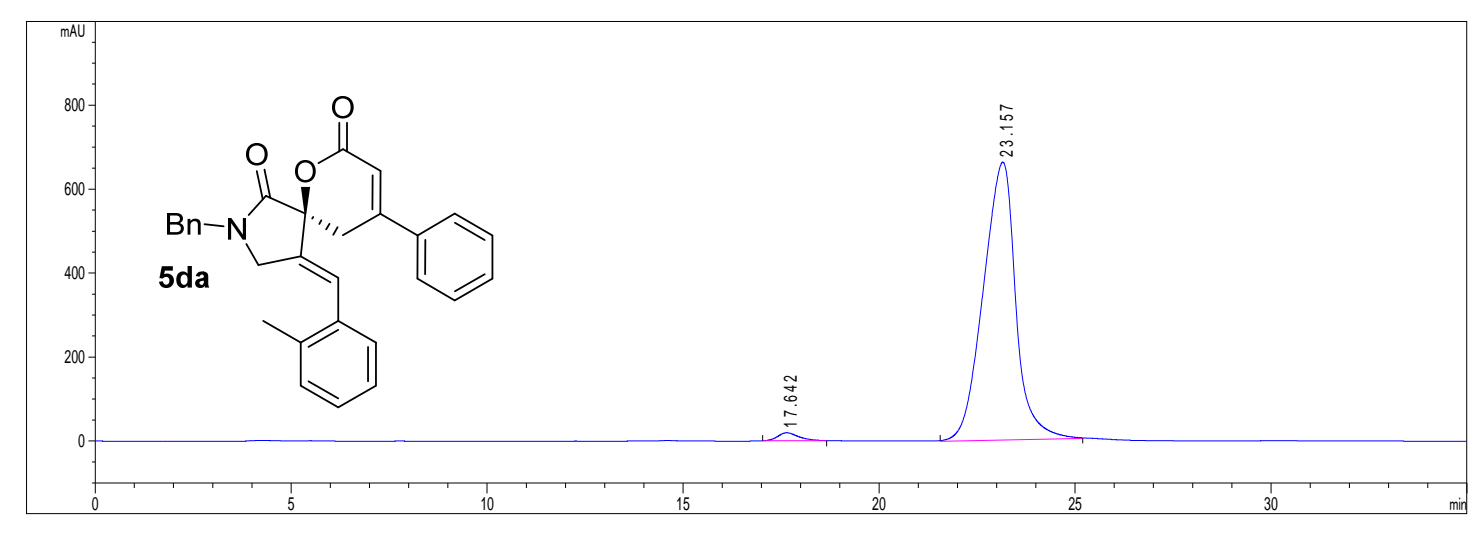

\begin{tabular}{|l|l|l|l|}
\hline & Retention & Area & Area\% \\
\hline 1 & 17.642 & 719.60040 & 1.8529 \\
\hline 2 & 23.157 & $3.81160 \mathrm{e} 4$ & 98.1471 \\
\hline
\end{tabular}




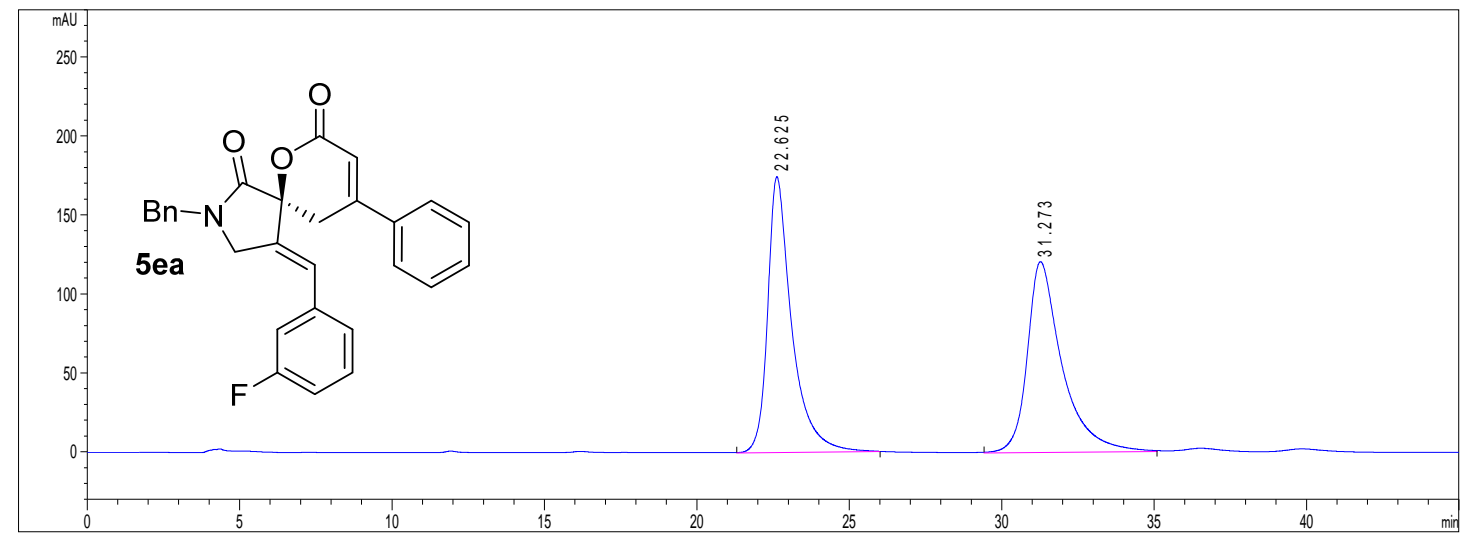

\begin{tabular}{|l|l|l|l|}
\hline & Retention & Area & Area\% \\
\hline 1 & 22.625 & 9601.70703 & 50.0473 \\
\hline 2 & 31.273 & 9583.57227 & 49.9527 \\
\hline
\end{tabular}

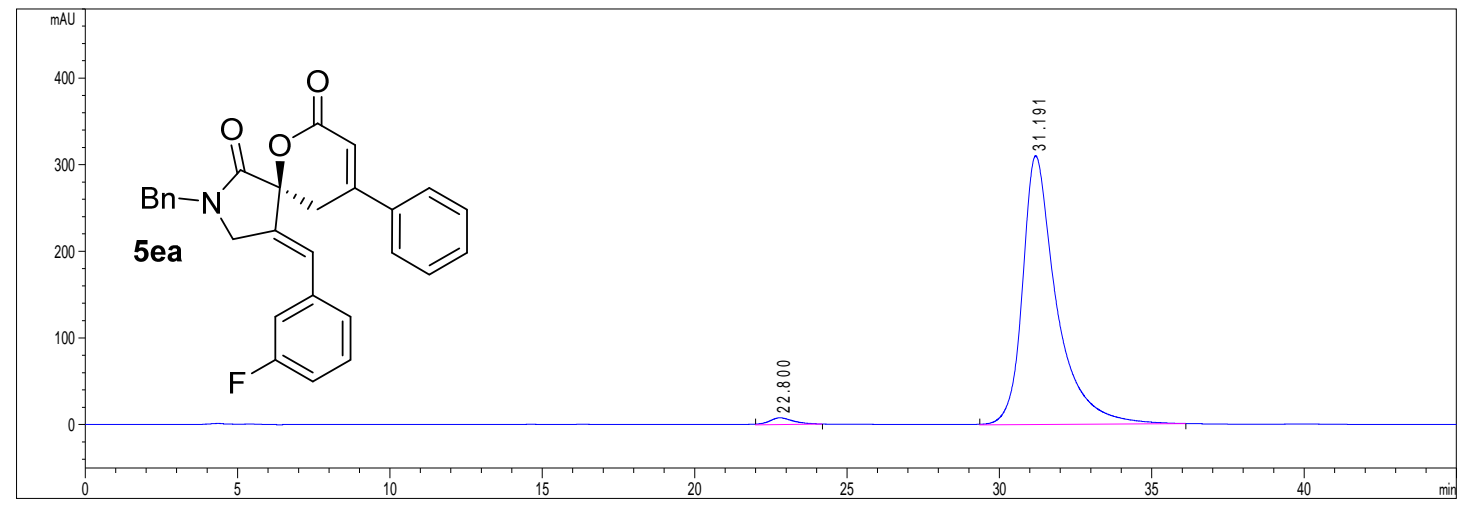

\begin{tabular}{|l|l|l|l|}
\hline & Retention & Area & Area\% \\
\hline 1 & 22.800 & 407.83154 & 1.6648 \\
\hline 2 & 31.191 & $2.40901 \mathrm{e} 4$ & 98.3352 \\
\hline
\end{tabular}




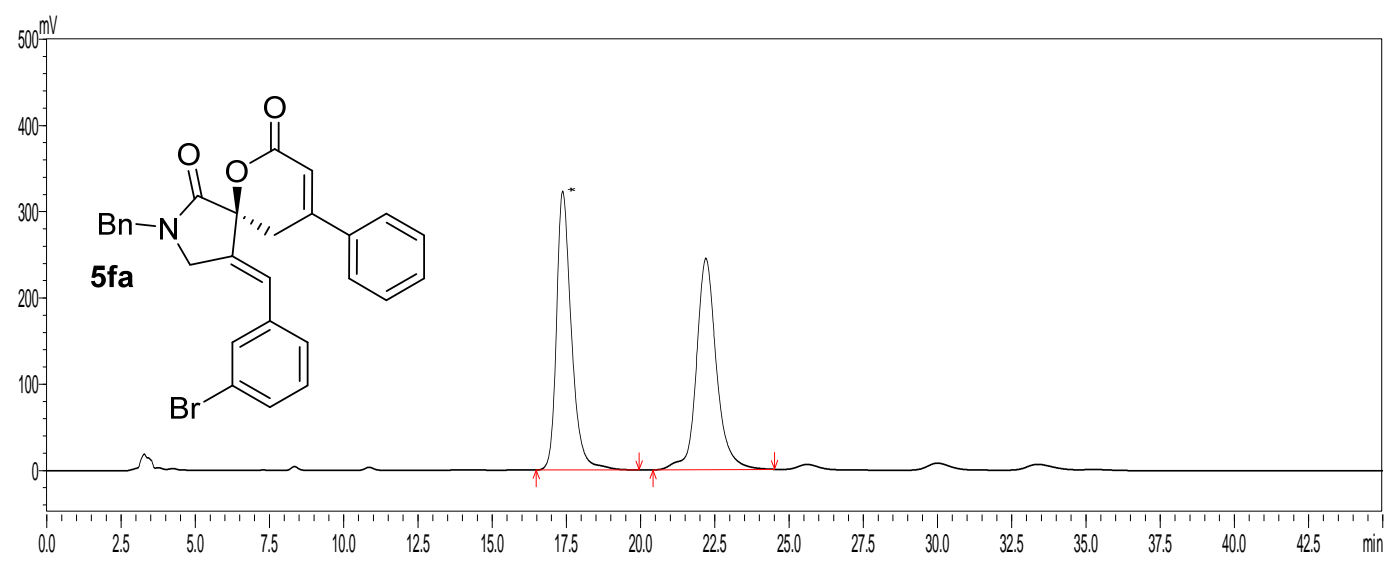

\begin{tabular}{|l|l|l|l|}
\hline & Retention & Area & Area\% \\
\hline 1 & 17.374 & 11277707 & 49.549 \\
\hline 2 & 22.199 & 11483188 & 50.451 \\
\hline
\end{tabular}

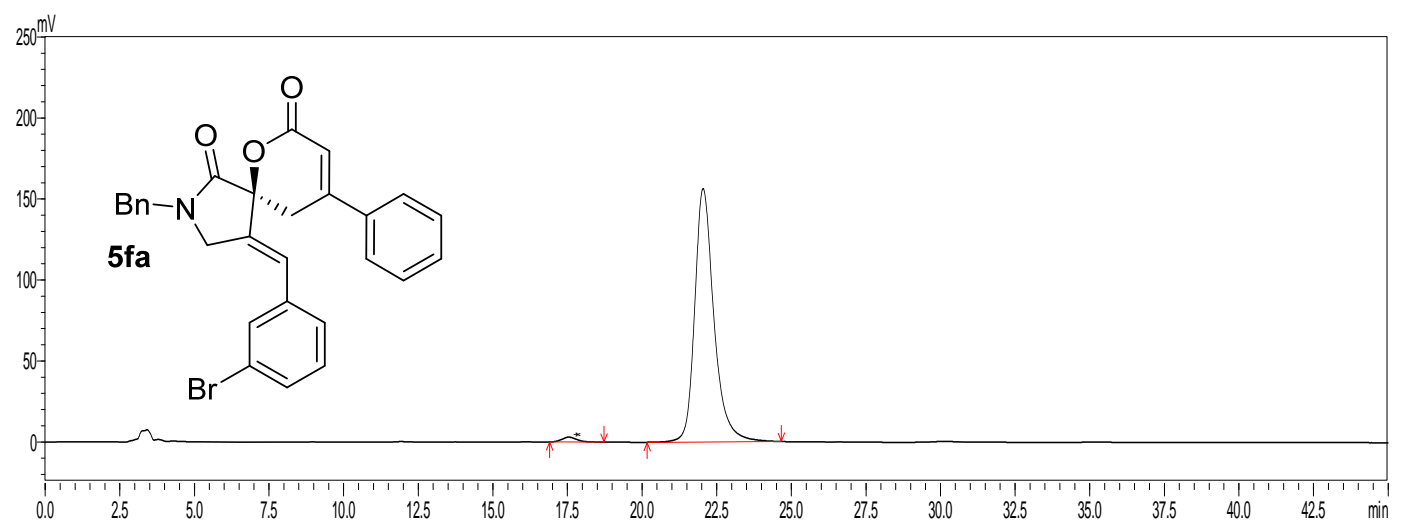

\begin{tabular}{|l|l|l|l|}
\hline & Retention & Area & Area\% \\
\hline 1 & 17.545 & 106656 & 1.497 \\
\hline 2 & 22.046 & 7020154 & 98.503 \\
\hline
\end{tabular}




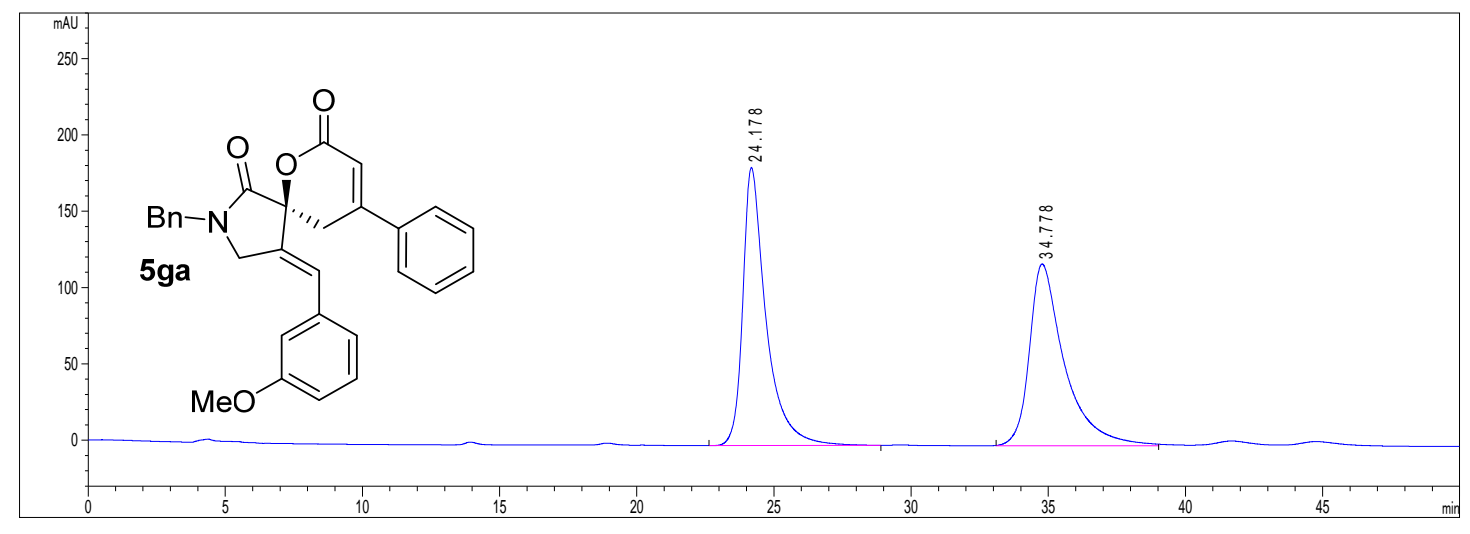

\begin{tabular}{|l|l|l|l|}
\hline & Retention & Area & Area\% \\
\hline 1 & 24.178 & $1.07577 \mathrm{e} 4$ & 50.3175 \\
\hline 2 & 34.778 & $1.06220 \mathrm{e} 4$ & 49.6825 \\
\hline
\end{tabular}

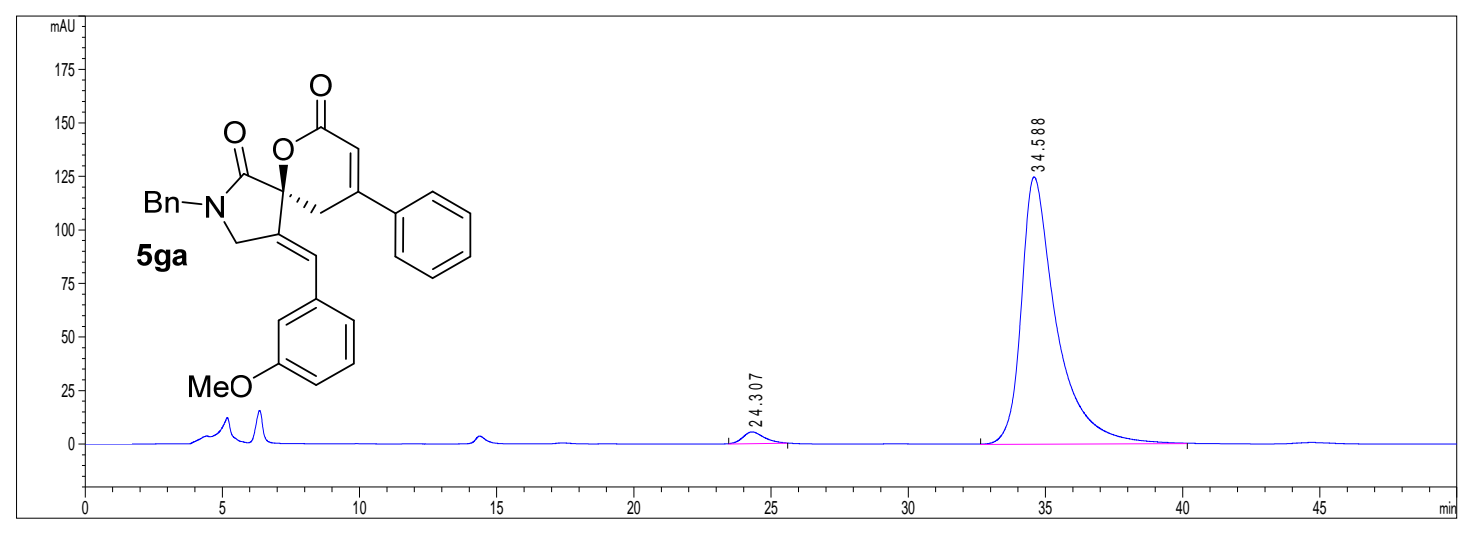

\begin{tabular}{|l|l|l|l|}
\hline & Retention & Area & Area\% \\
\hline 1 & 24.307 & 303.24237 & 2.6582 \\
\hline 2 & 34.588 & $1.11047 \mathrm{e} 4$ & 97.3418 \\
\hline
\end{tabular}




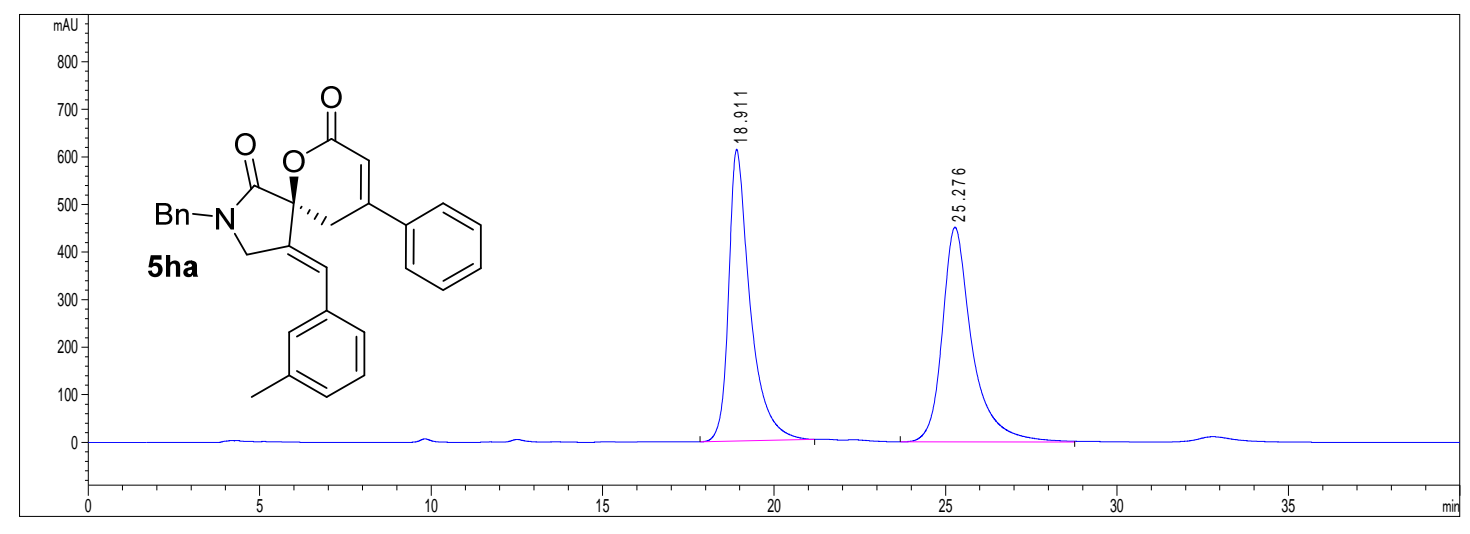

\begin{tabular}{|l|l|l|l|}
\hline & Retention & Area & Area\% \\
\hline 1 & 18.911 & $2.67185 \mathrm{e} 4$ & 49.4466 \\
\hline 2 & 25.276 & $2.73166 \mathrm{e} 4$ & 50.5534 \\
\hline
\end{tabular}

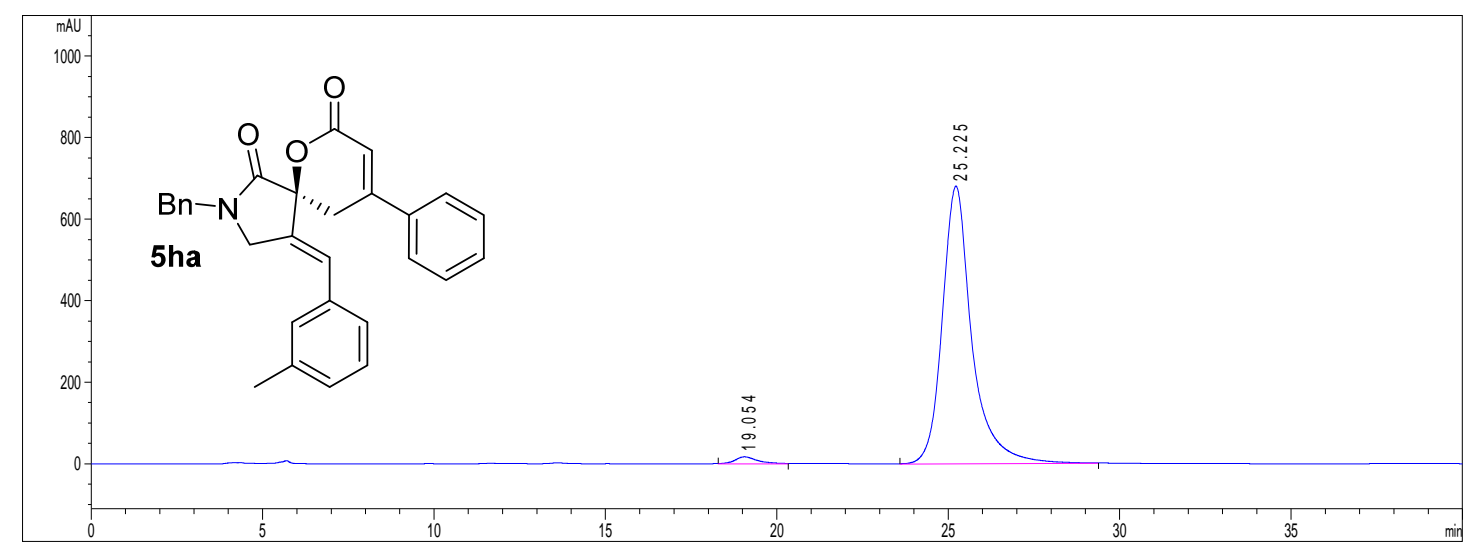

\begin{tabular}{|l|l|l|l|}
\hline & Retention & Area & Area\% \\
\hline 1 & 19.054 & 795.80243 & 1.9079 \\
\hline 2 & 25.225 & $4.09142 \mathrm{e} 4$ & 98.0921 \\
\hline
\end{tabular}




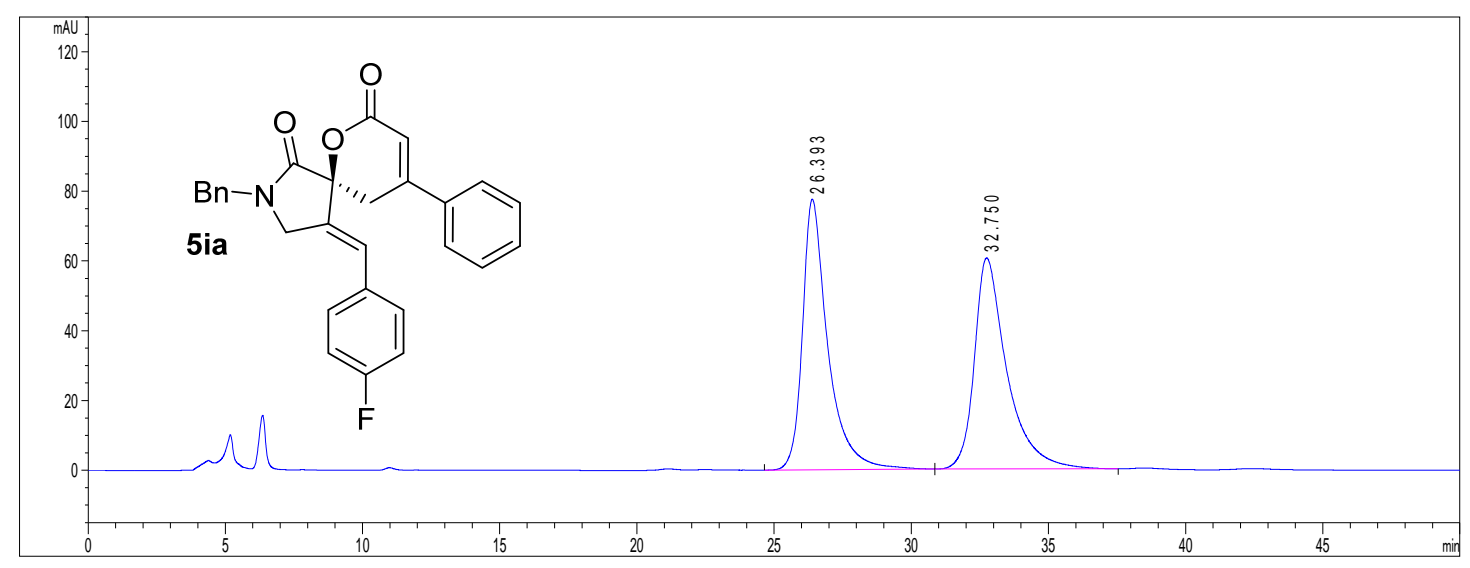

\begin{tabular}{|l|l|l|l|}
\hline & Retention & Area & Area\% \\
\hline 1 & 26.393 & 4988.89648 & 49.9956 \\
\hline 2 & 32.750 & 4989.78418 & 50.0044 \\
\hline
\end{tabular}

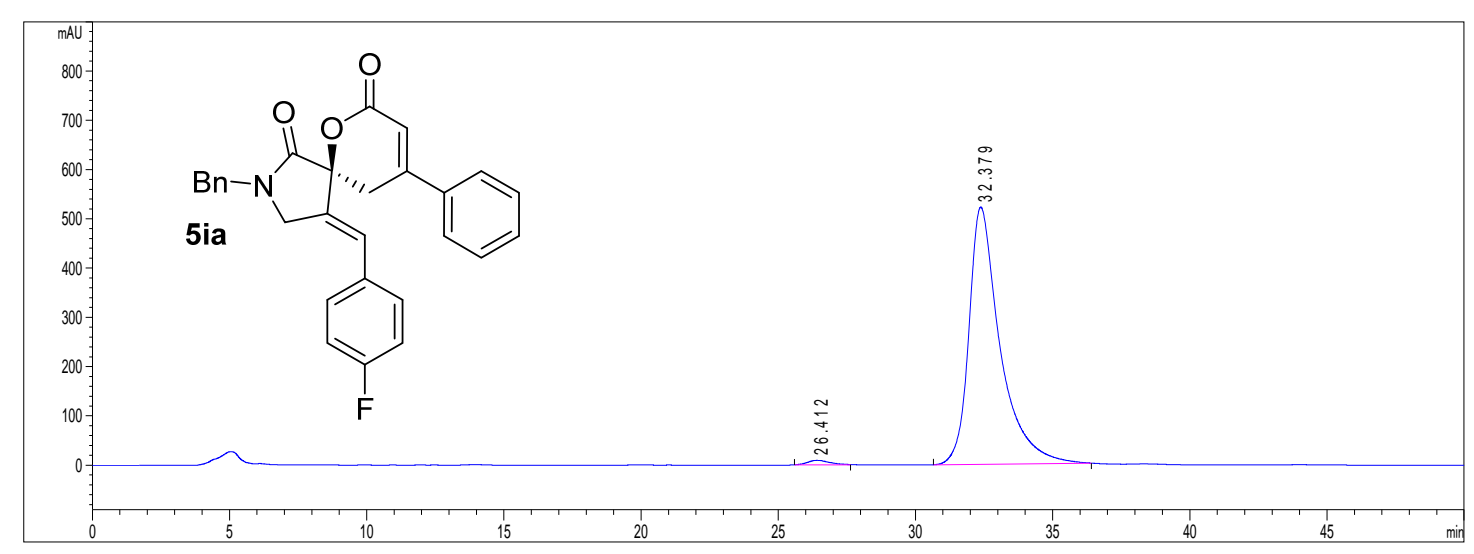

\begin{tabular}{|l|l|l|l|}
\hline & Retention & Area & Area\% \\
\hline 1 & 26.412 & 517.28937 & 1.2387 \\
\hline 2 & 32.379 & $4.12425 \mathrm{e} 4$ & 98.7613 \\
\hline
\end{tabular}




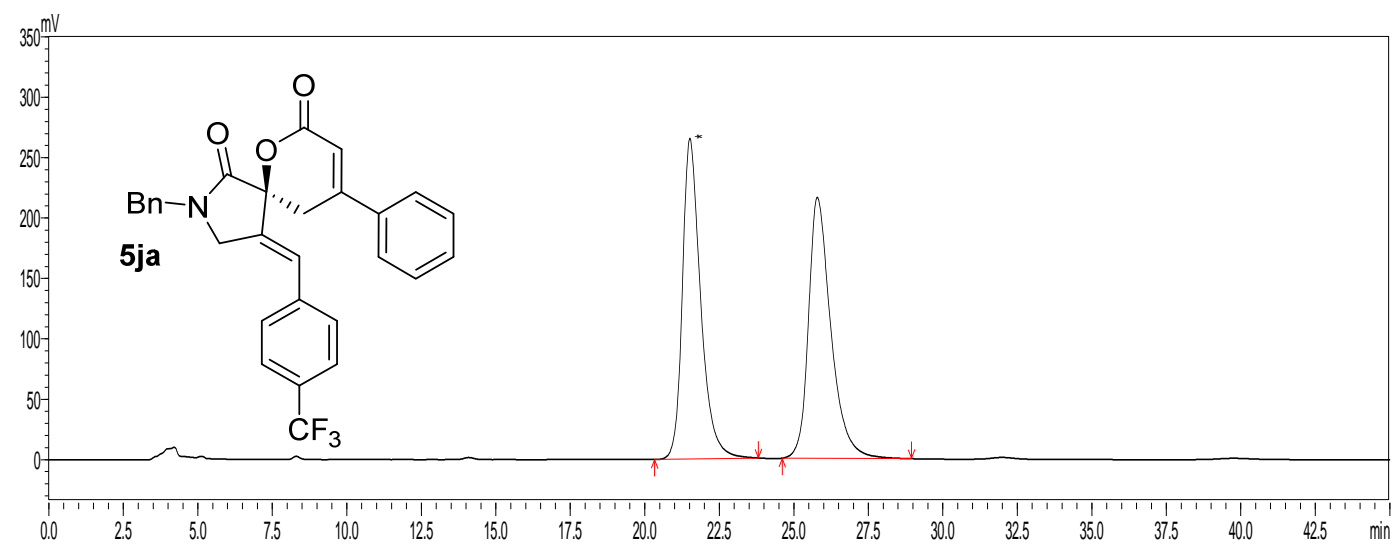

\begin{tabular}{|l|l|l|l|}
\hline & Retention & Area & Area\% \\
\hline 1 & 21.511 & 11306793 & 50.037 \\
\hline 2 & 25.792 & 11289960 & 49.963 \\
\hline
\end{tabular}

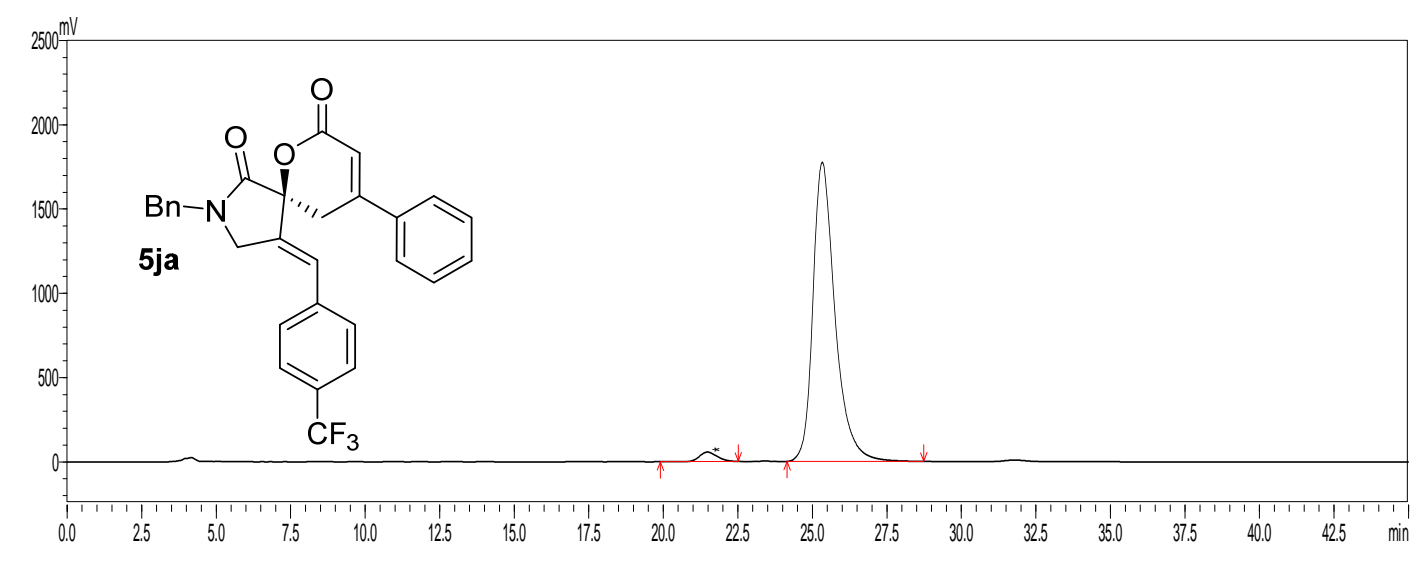

\begin{tabular}{|l|l|l|l|}
\hline & Retention & Area & Area\% \\
\hline 1 & 21.477 & 2335587 & 2.485 \\
\hline 2 & 25.333 & 91647795 & 97.515 \\
\hline
\end{tabular}




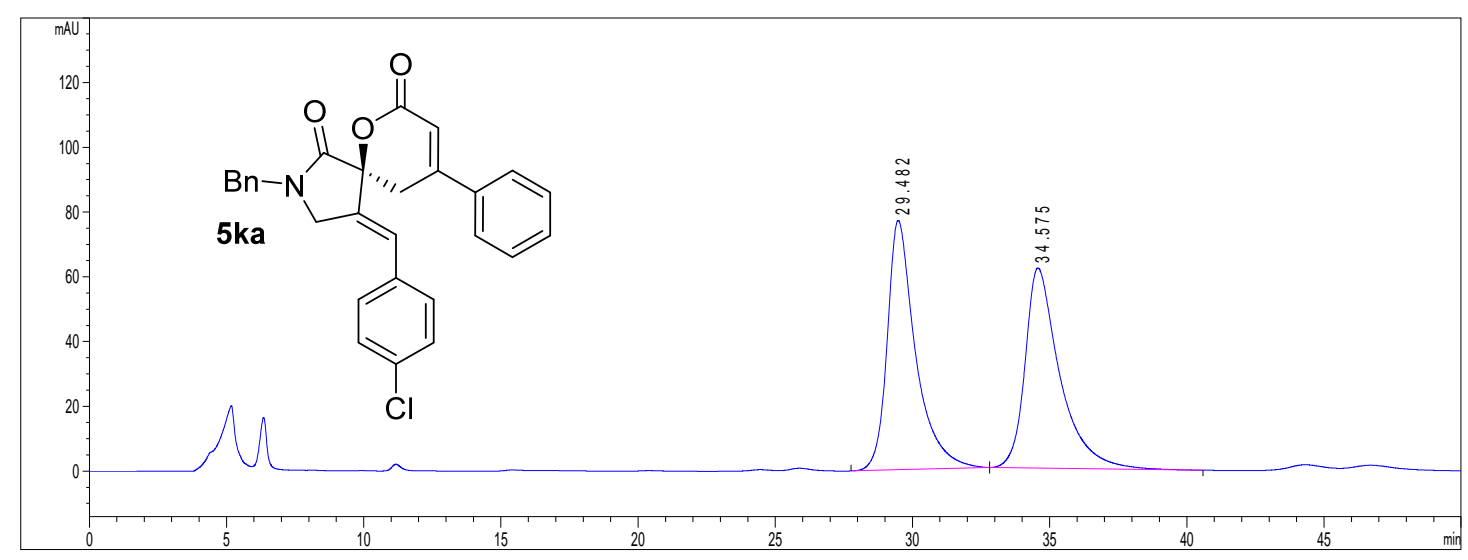

\begin{tabular}{|l|l|l|l|}
\hline & Retention & Area & Area\% \\
\hline 1 & 29.482 & 5527.54150 & 50.3552 \\
\hline 2 & 29.482 & 5449.54932 & 49.6448 \\
\hline
\end{tabular}

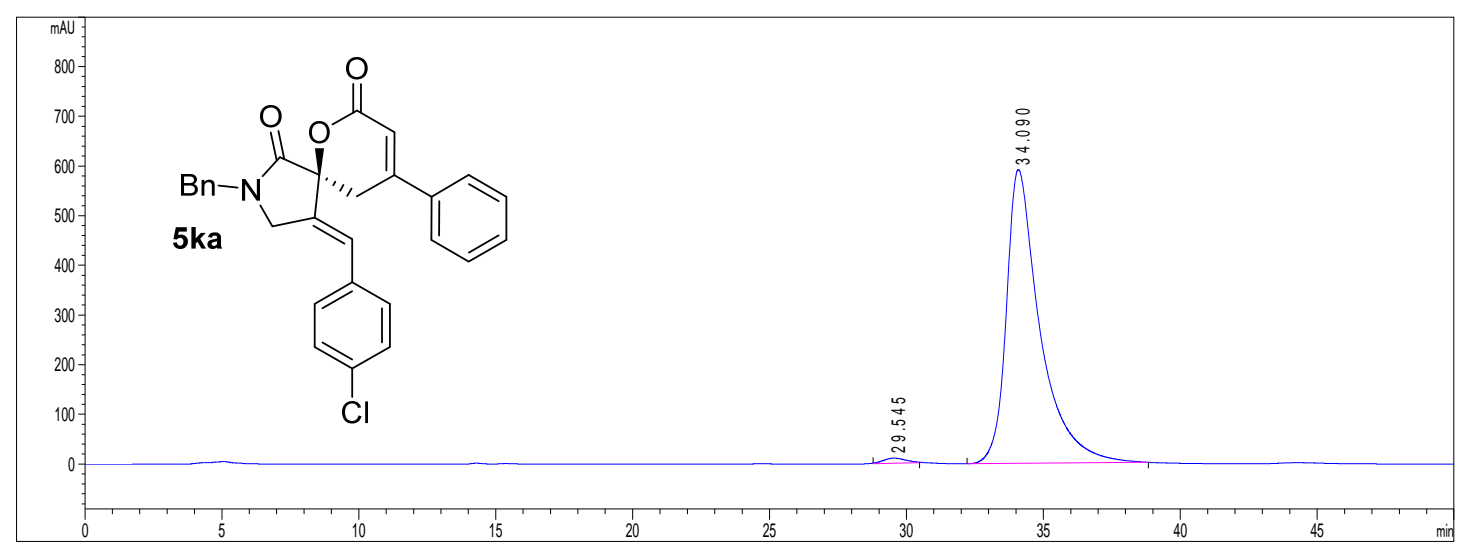

\begin{tabular}{|l|l|l|l|}
\hline & Retention & Area & Area\% \\
\hline 1 & 29.545 & 572.65070 & 1.1179 \\
\hline 2 & 34.090 & $5.06544 \mathrm{e} 4$ & 98.8821 \\
\hline
\end{tabular}




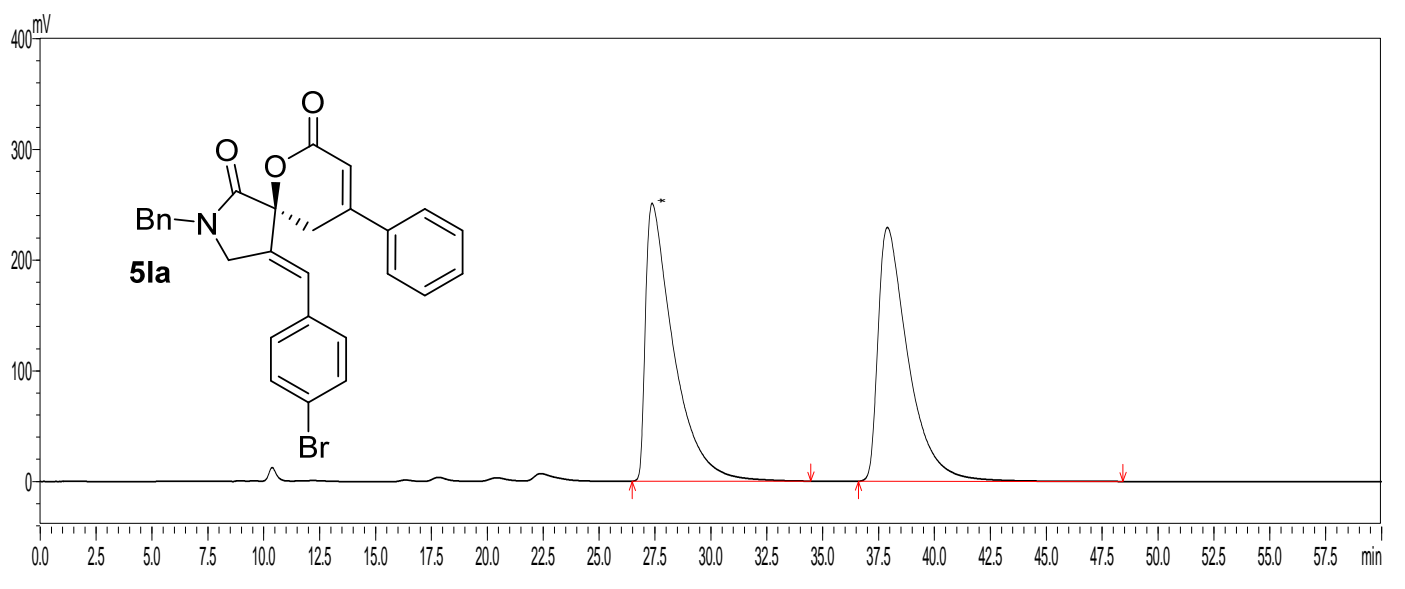

\begin{tabular}{|l|l|l|l|}
\hline & Retention & Area & Area\% \\
\hline 1 & 27.374 & 21955044 & 50.479 \\
\hline 2 & 37.895 & 21538627 & 49.521 \\
\hline
\end{tabular}

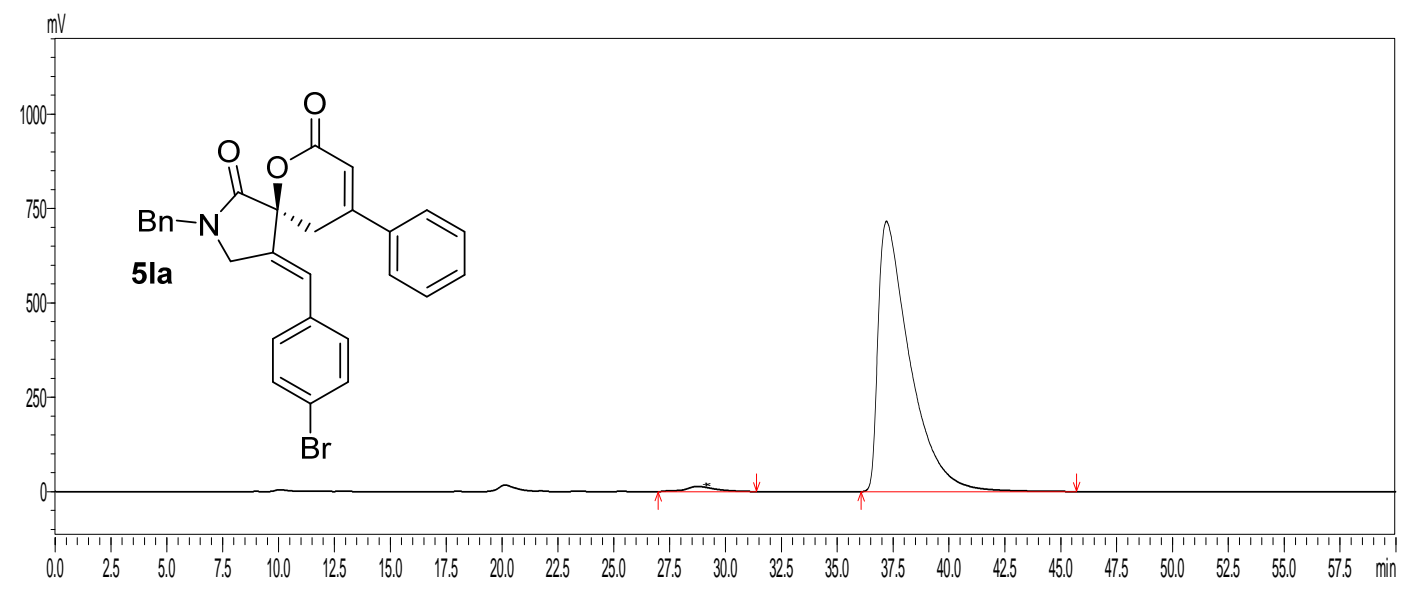

\begin{tabular}{|l|l|l|l|}
\hline & Retention & Area & Area\% \\
\hline 1 & 28.755 & 1266062 & 1.755 \\
\hline 2 & 37.202 & 70877682 & 98.245 \\
\hline
\end{tabular}

S148 


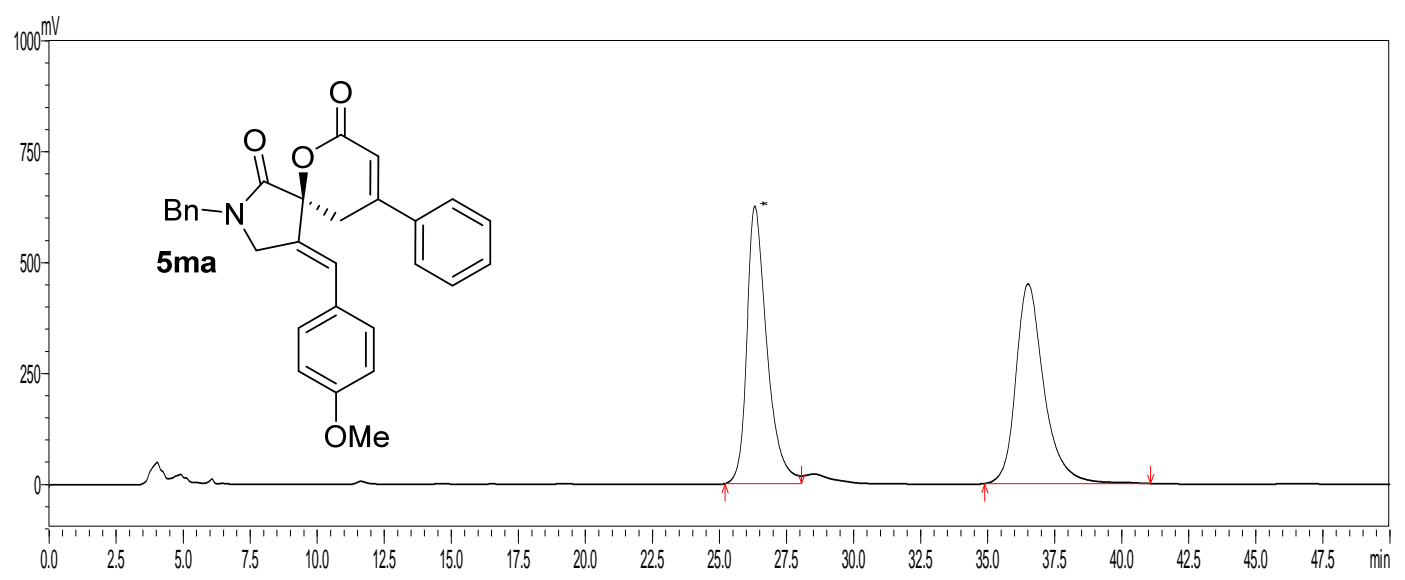

\begin{tabular}{|l|l|l|l|}
\hline & Retention & Area & Area\% \\
\hline 1 & 26.316 & 32546734 & 49.761 \\
\hline 2 & 36.508 & 32859594 & 50.239 \\
\hline
\end{tabular}

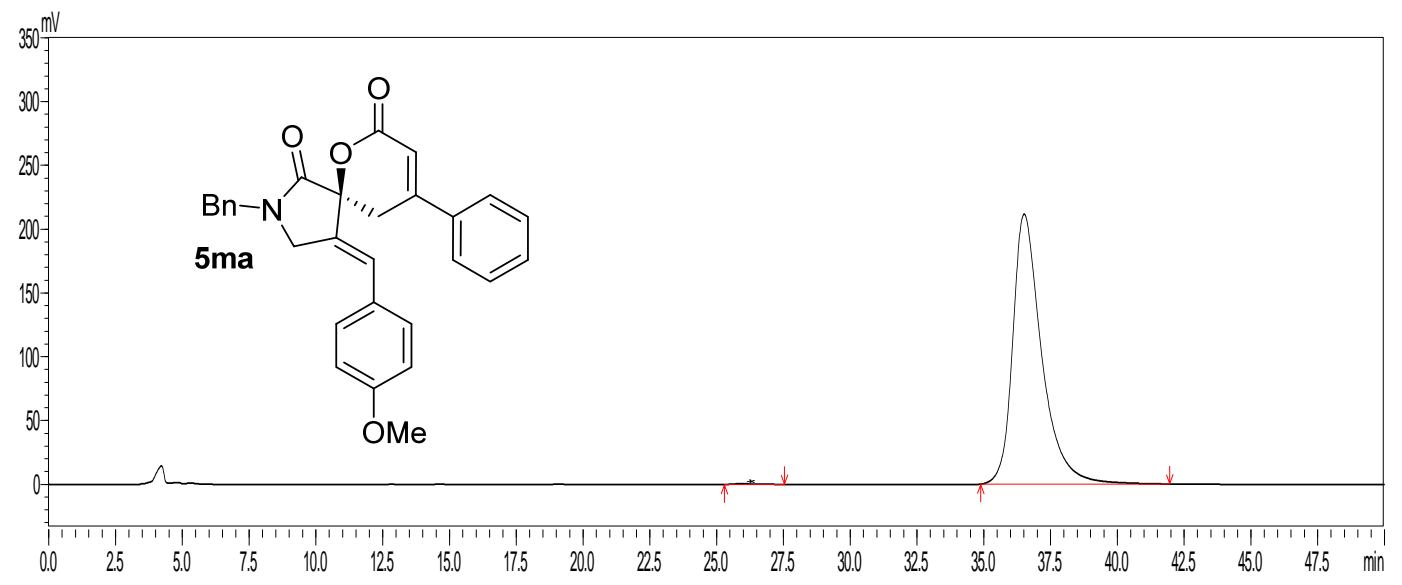

\begin{tabular}{|l|l|l|l|}
\hline & Retention & Area & Area\% \\
\hline 1 & 25.918 & 38071 & 0.238 \\
\hline 2 & 36.512 & 15959965 & 99.762 \\
\hline
\end{tabular}




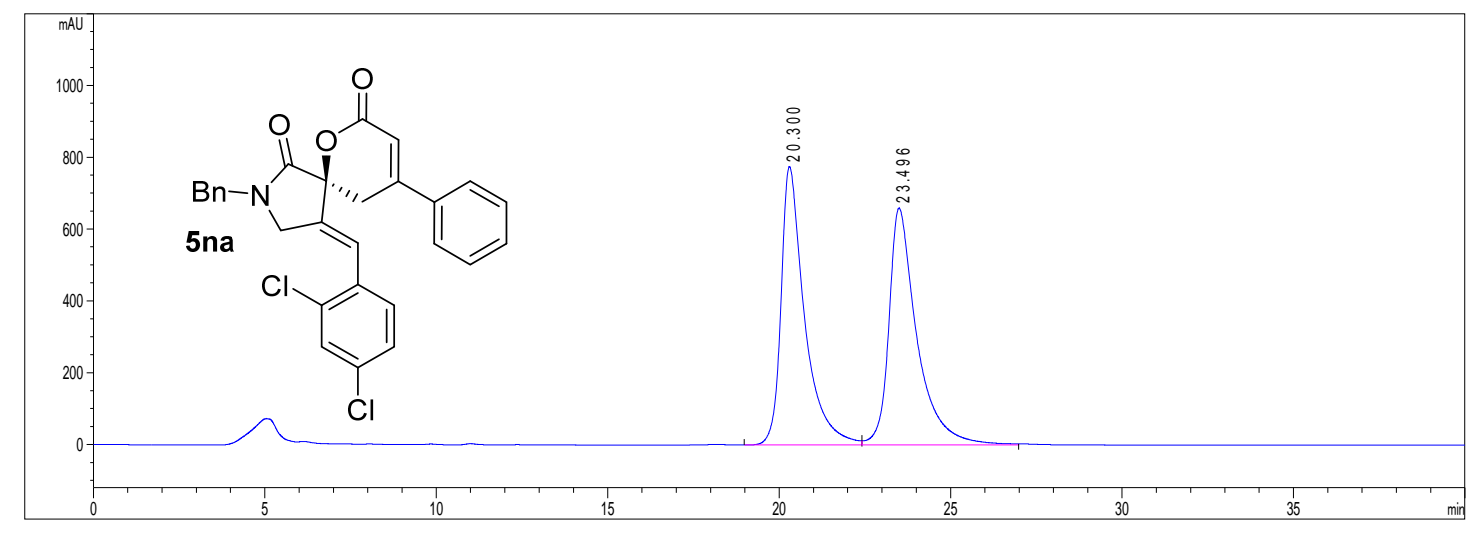

\begin{tabular}{|l|l|l|l|}
\hline & Retention & Area & Area\% \\
\hline 1 & 20.300 & $3.69347 \mathrm{e} 4$ & 49.6583 \\
\hline 2 & 23.496 & $3.74430 \mathrm{e} 4$ & 50.3417 \\
\hline
\end{tabular}

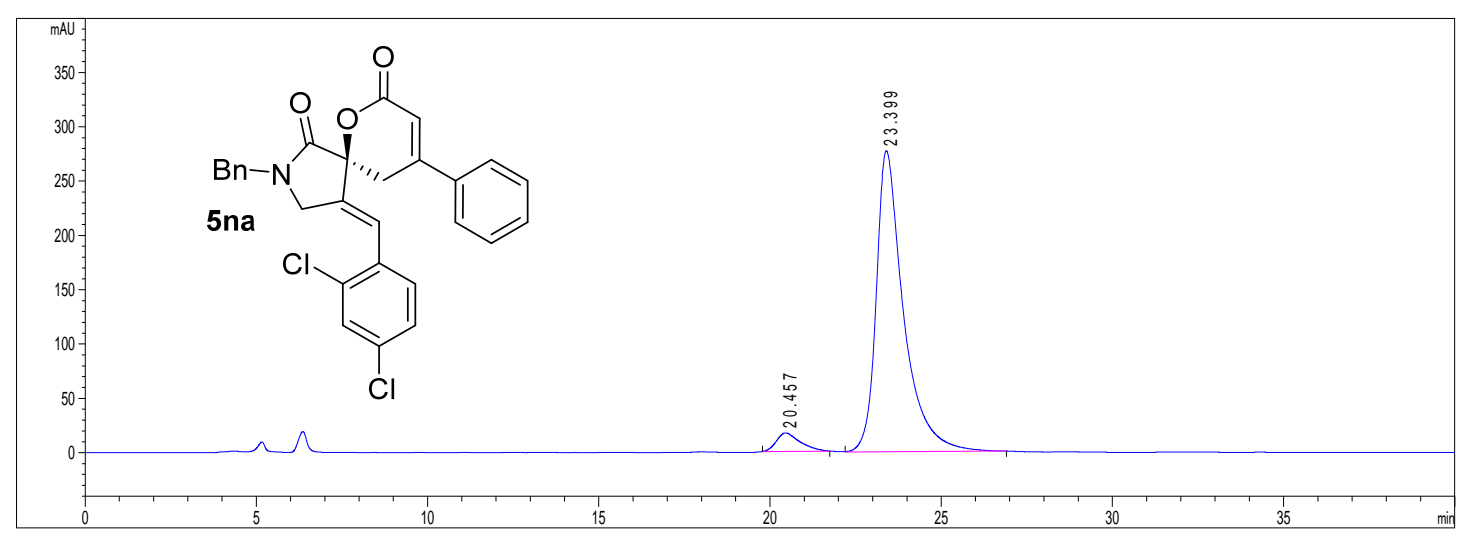

\begin{tabular}{|l|l|l|l|}
\hline & Retention & Area & Area\% \\
\hline 1 & 20.457 & 825.64404 & 5.1025 \\
\hline 2 & 23.399 & $1.53556 \mathrm{e} 4$ & 94.8975 \\
\hline
\end{tabular}




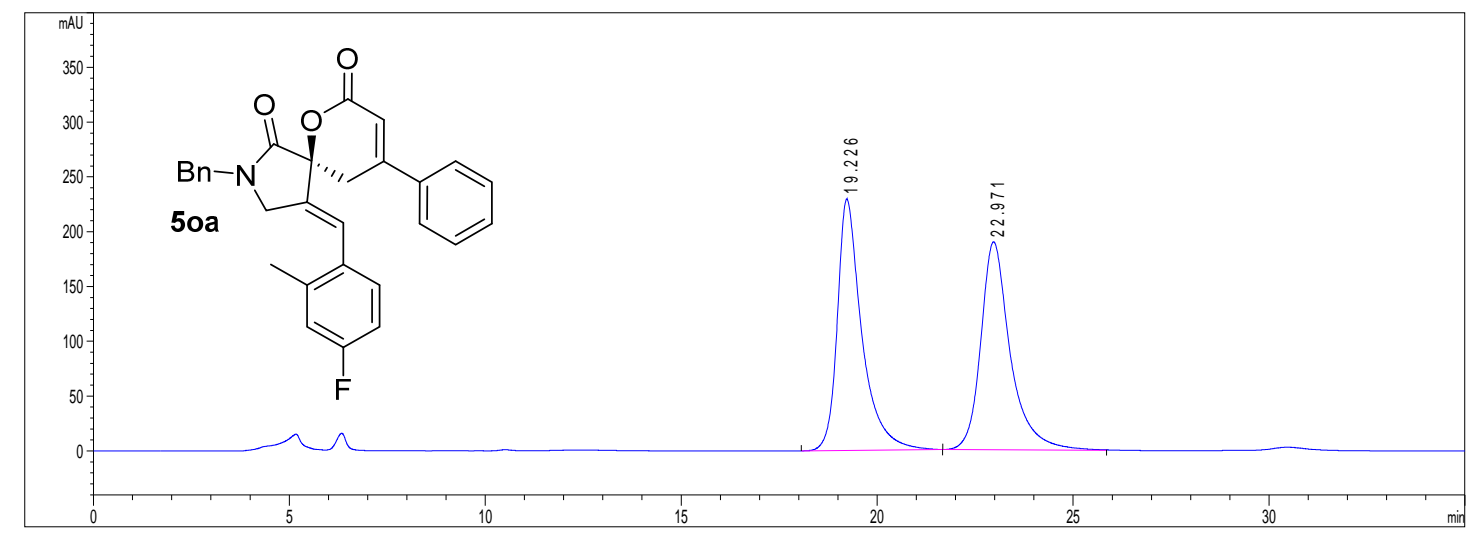

\begin{tabular}{|l|l|l|l|}
\hline & Retention & Area & Area\% \\
\hline 1 & 19.226 & 9973.15137 & 50.0773 \\
\hline 2 & 22.971 & 9942.37207 & 49.9227 \\
\hline
\end{tabular}

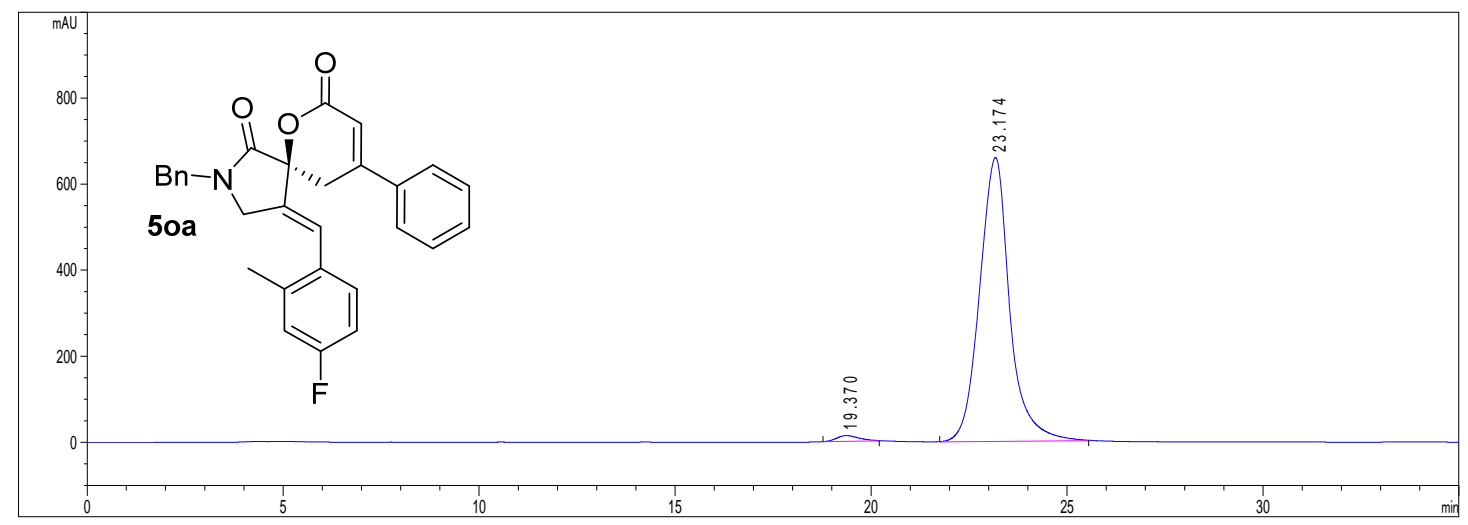

\begin{tabular}{|l|l|l|l|}
\hline & Retention & Area & Area $\%$ \\
\hline 1 & 19.370 & 544.69800 & 1.5279 \\
\hline 2 & 23.174 & $3.51065 \mathrm{e} 4$ & 98.4721 \\
\hline
\end{tabular}




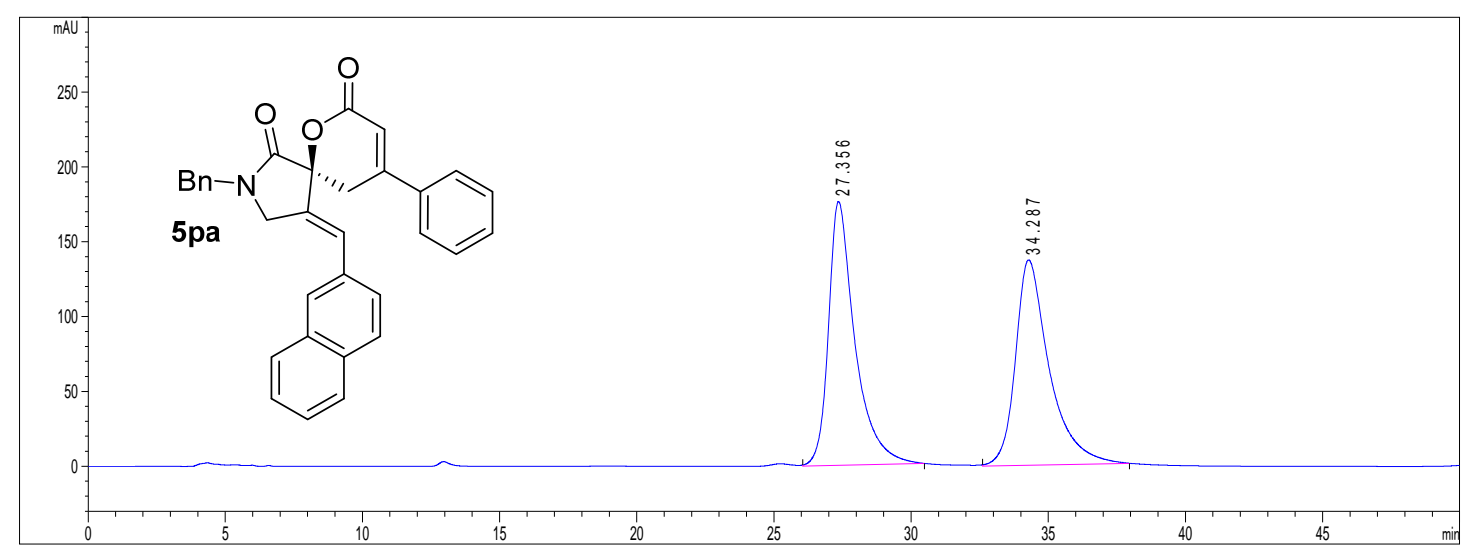

\begin{tabular}{|l|l|l|l|}
\hline & Retention & Area & Area $\%$ \\
\hline 1 & 27.356 & $1.18016 \mathrm{e} 4$ & 50.1536 \\
\hline 2 & 34.287 & $1.17293 \mathrm{e} 4$ & 49.8464 \\
\hline
\end{tabular}

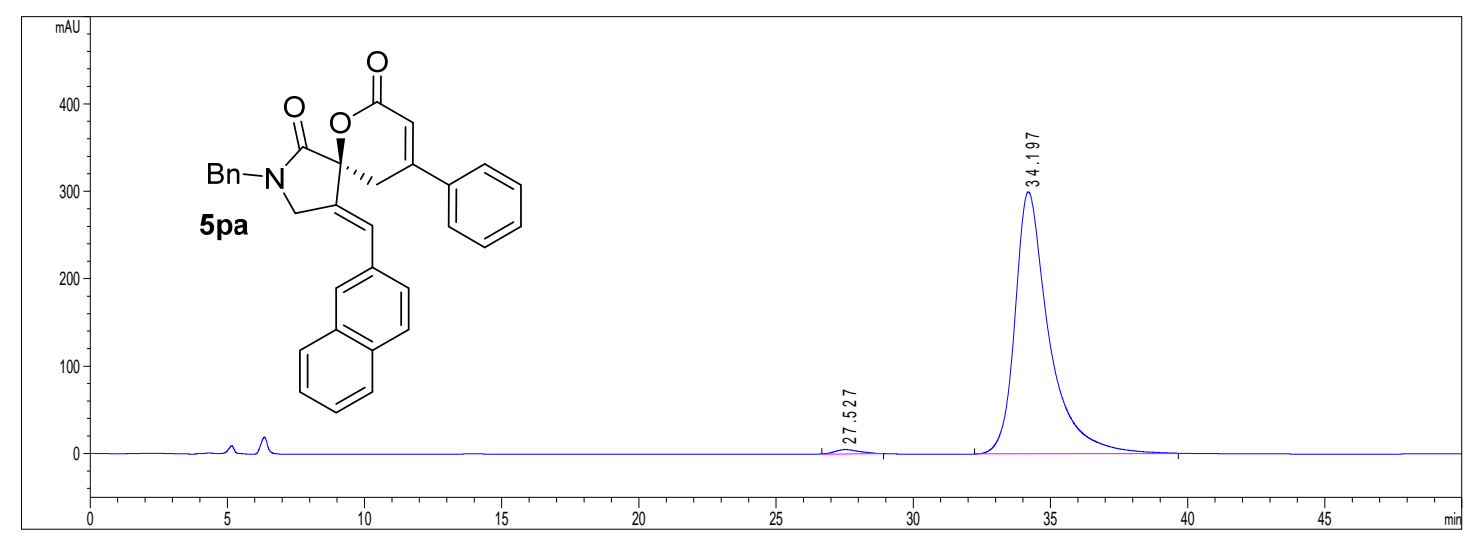

\begin{tabular}{|l|l|l|l|}
\hline & Retention & Area & Area\% \\
\hline 1 & 27.527 & 300.68250 & 1.1479 \\
\hline 2 & 34.197 & $2.58931 \mathrm{e} 4$ & 98.8521 \\
\hline
\end{tabular}




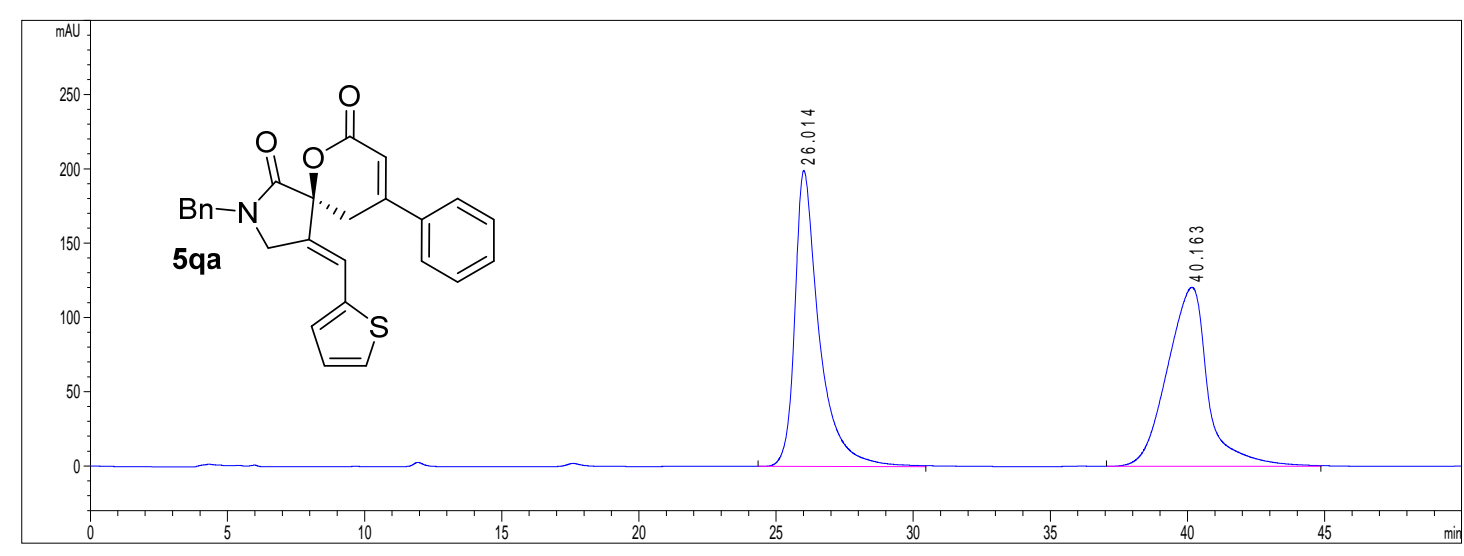

\begin{tabular}{|l|l|l|l|}
\hline & Retention & Area & Area $\%$ \\
\hline 1 & 26.014 & $1.23542 \mathrm{e} 4$ & 49.7656 \\
\hline 2 & 40.163 & $1.24706 \mathrm{e} 4$ & 50.2344 \\
\hline
\end{tabular}

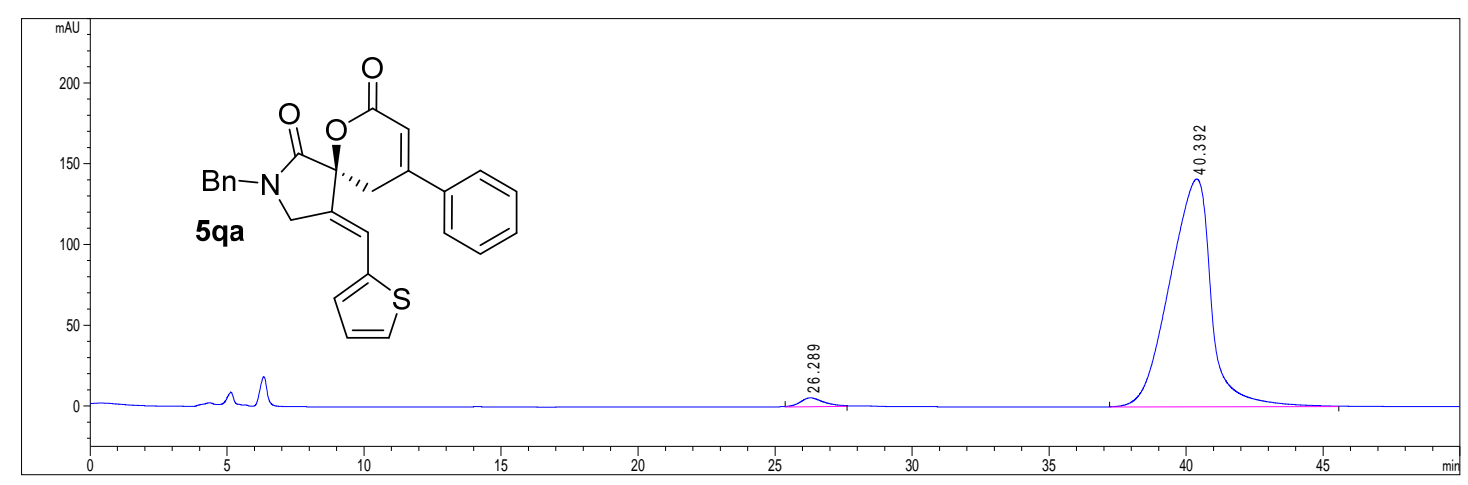

\begin{tabular}{|l|l|l|l|}
\hline & Retention & Area & Area\% \\
\hline 1 & 26.289 & 331.18304 & 2.1699 \\
\hline 2 & 40.392 & $1.49313 \mathrm{e} 4$ & 97.8301 \\
\hline
\end{tabular}




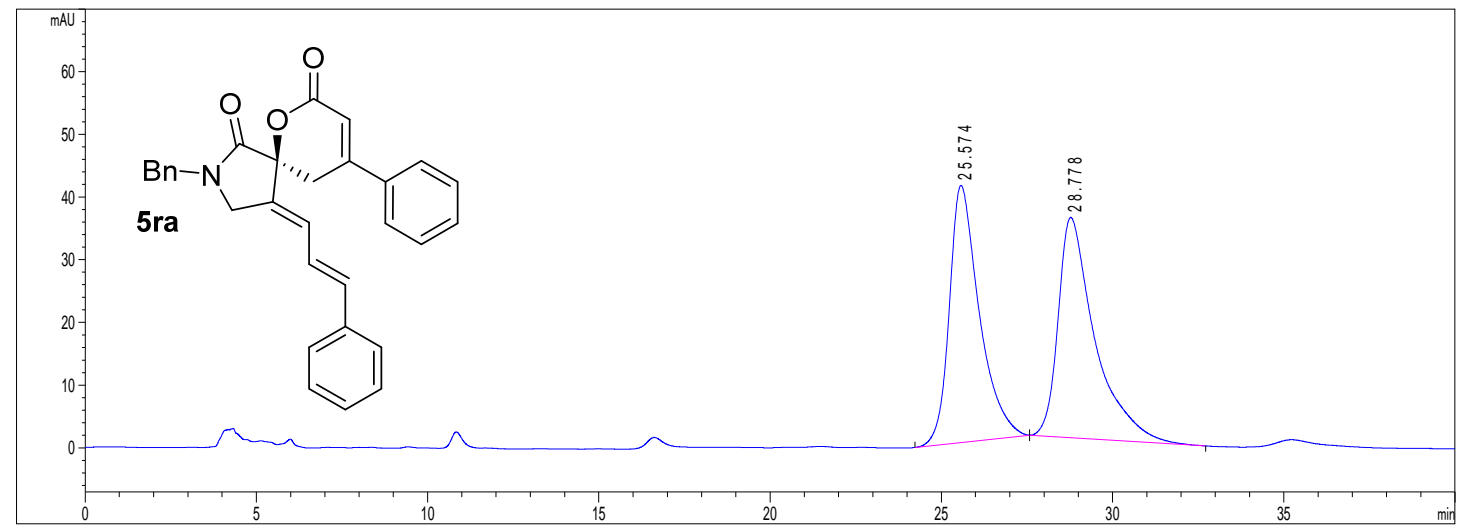

\begin{tabular}{|l|l|l|l|}
\hline & Retention & Area & Area\% \\
\hline 1 & 25.574 & 2494.07471 & 48.7723 \\
\hline 2 & 28.778 & 2619.63184 & 51.2277 \\
\hline
\end{tabular}

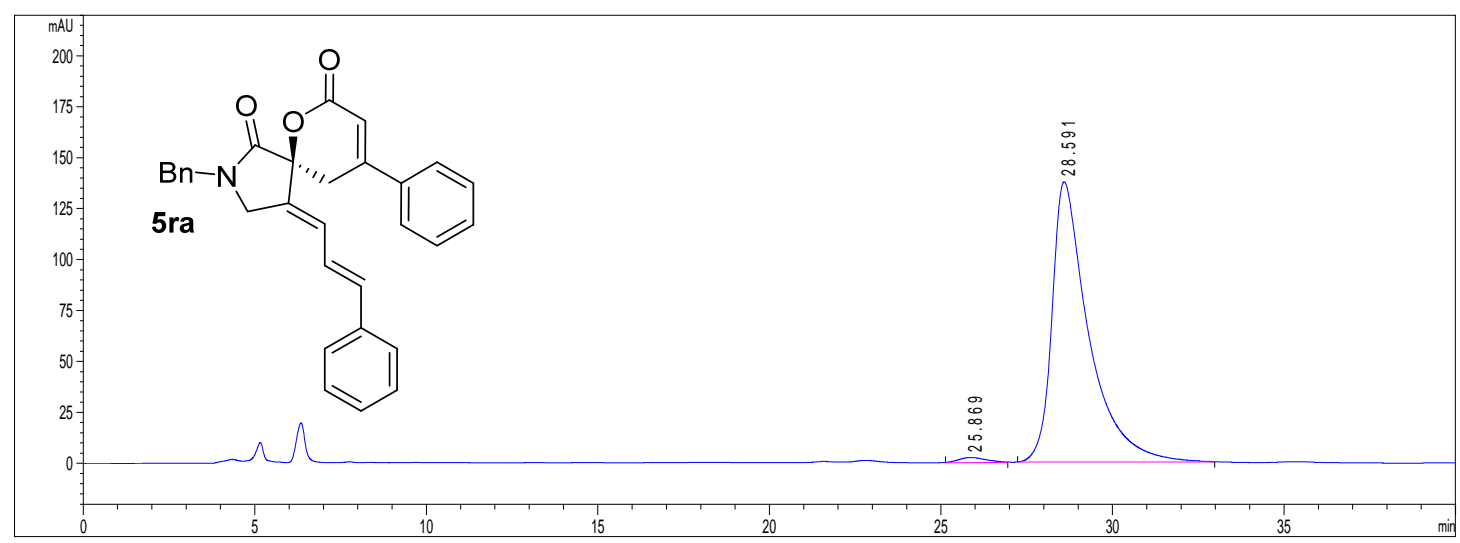

\begin{tabular}{|l|l|l|l|}
\hline & Retention & Area & Area\% \\
\hline 1 & 25.869 & 143.29050 & 1.3806 \\
\hline 2 & 28.591 & $1.02352 \mathrm{e} 4$ & 98.6194 \\
\hline
\end{tabular}




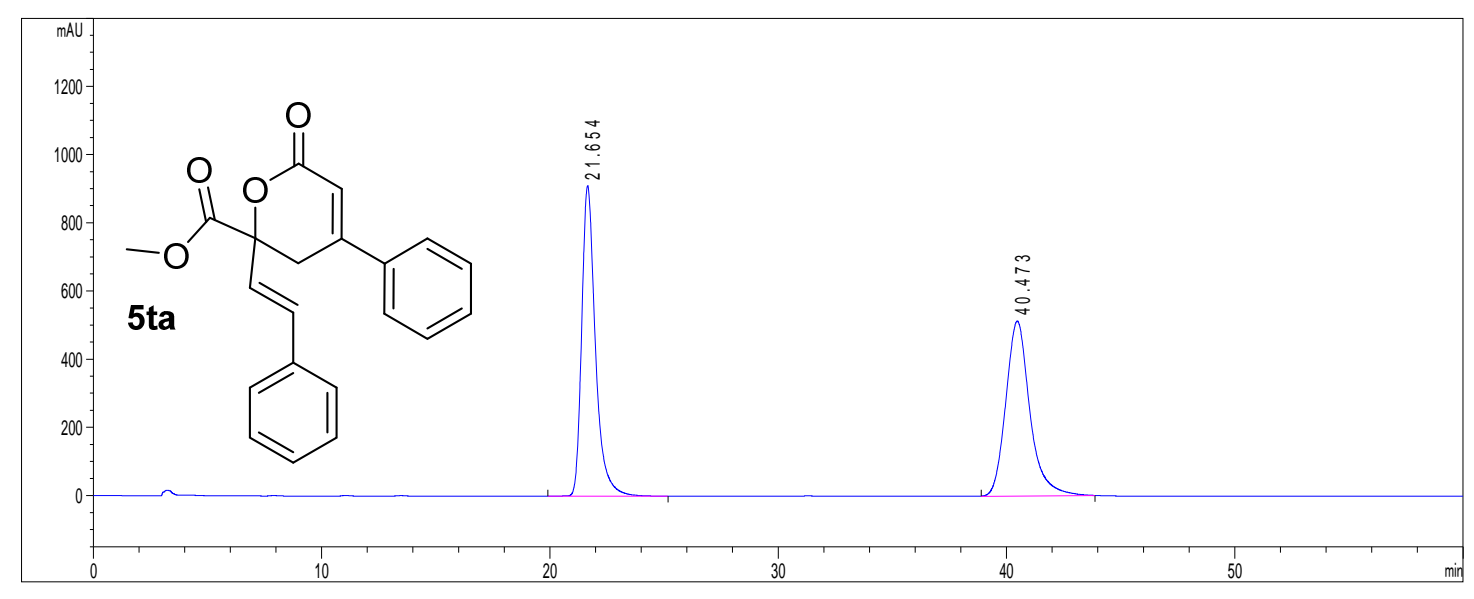

\begin{tabular}{|l|l|l|l|}
\hline & Retention & Area & Area $\%$ \\
\hline 1 & 21.654 & $3.80355 \mathrm{e} 4$ & 50.2341 \\
\hline 2 & 40.473 & $3.76810 \mathrm{e} 4$ & 49.7659 \\
\hline
\end{tabular}

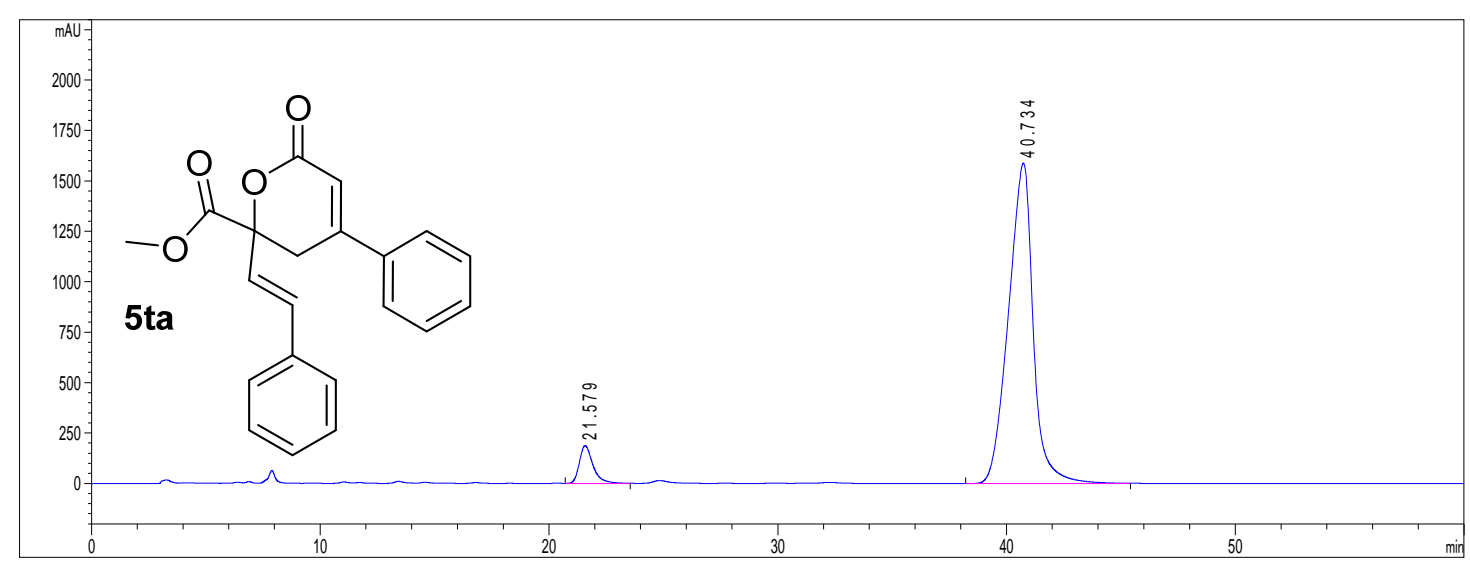

\begin{tabular}{|l|l|l|l|}
\hline & Retention & Area & Area\% \\
\hline 1 & 21.579 & 7722.76416 & 6.0867 \\
\hline 2 & 40.734 & $1.19156 \mathrm{e} 5$ & 93.9133 \\
\hline
\end{tabular}




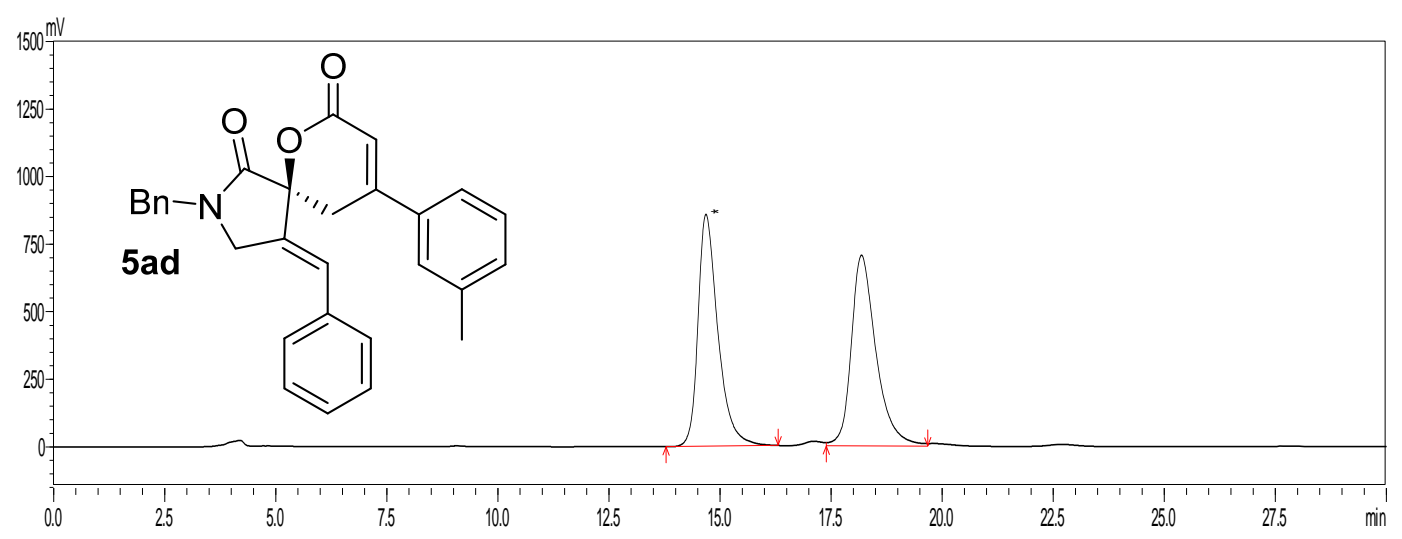

\begin{tabular}{|l|l|l|l|}
\hline & Retention & Area & Area\% \\
\hline 1 & 14.683 & 26772806 & 49.422 \\
\hline 2 & 18.184 & 27399439 & 50.578 \\
\hline
\end{tabular}

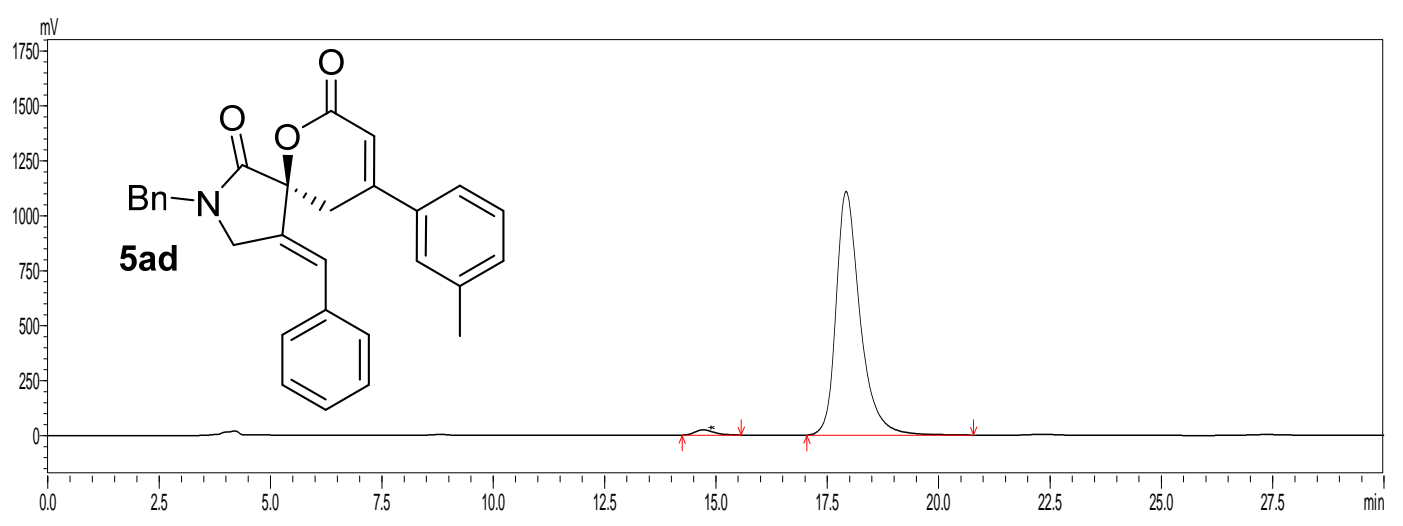

\begin{tabular}{|l|l|l|l|}
\hline & Retention & Area & Area\% \\
\hline 1 & 14.716 & 728289 & 1.723 \\
\hline 2 & 17.923 & 41552011 & 98.277 \\
\hline
\end{tabular}




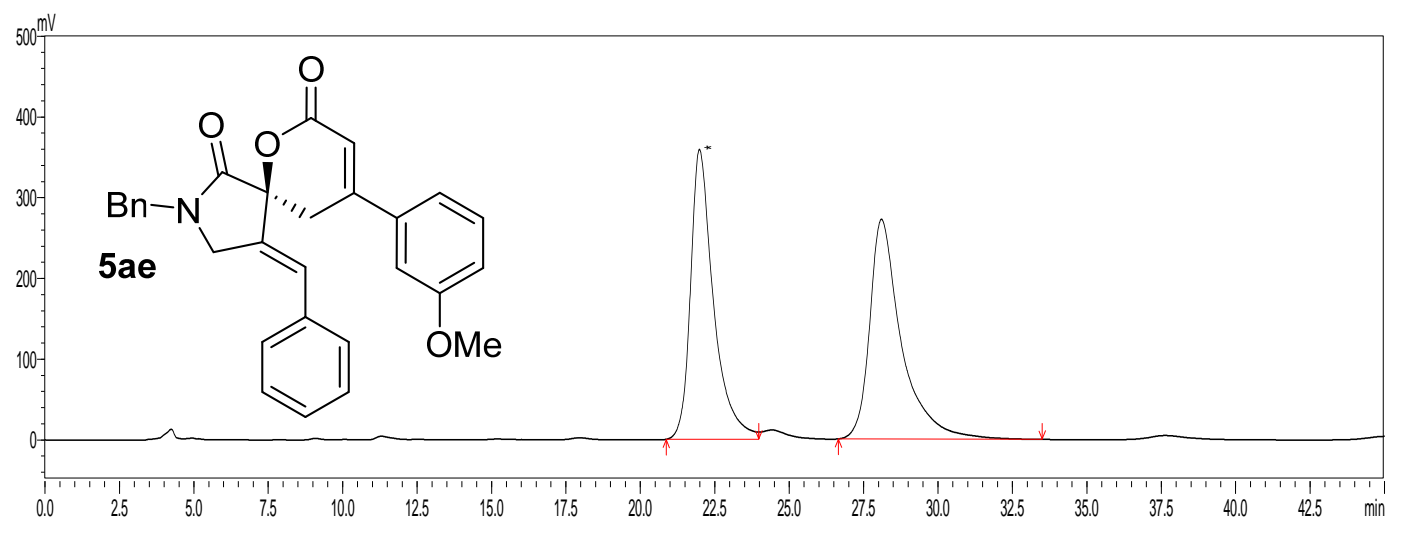

\begin{tabular}{|l|l|l|l|}
\hline & Retention & Area & Area\% \\
\hline 1 & 21.987 & 19315239 & 48.909 \\
\hline 2 & 28.101 & 20176903 & 51.091 \\
\hline
\end{tabular}

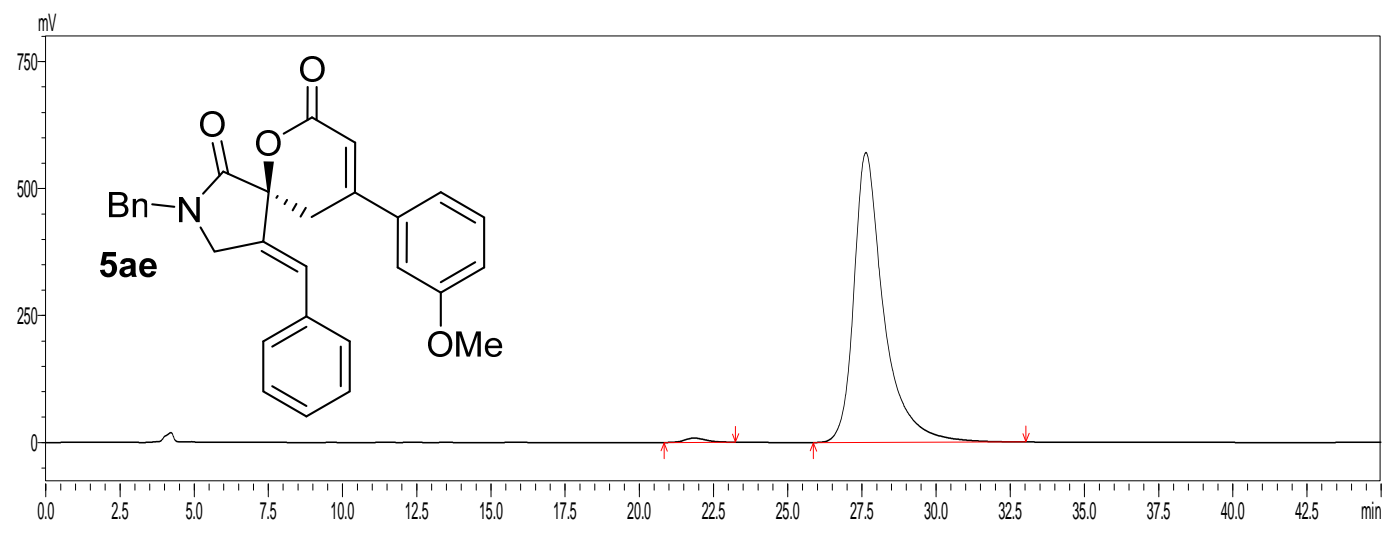

\begin{tabular}{|l|l|l|l|}
\hline & Retention & Area & Area $\%$ \\
\hline 1 & 21.848 & 455389 & 1.127 \\
\hline 2 & 27.635 & 39938102 & 98.873 \\
\hline
\end{tabular}




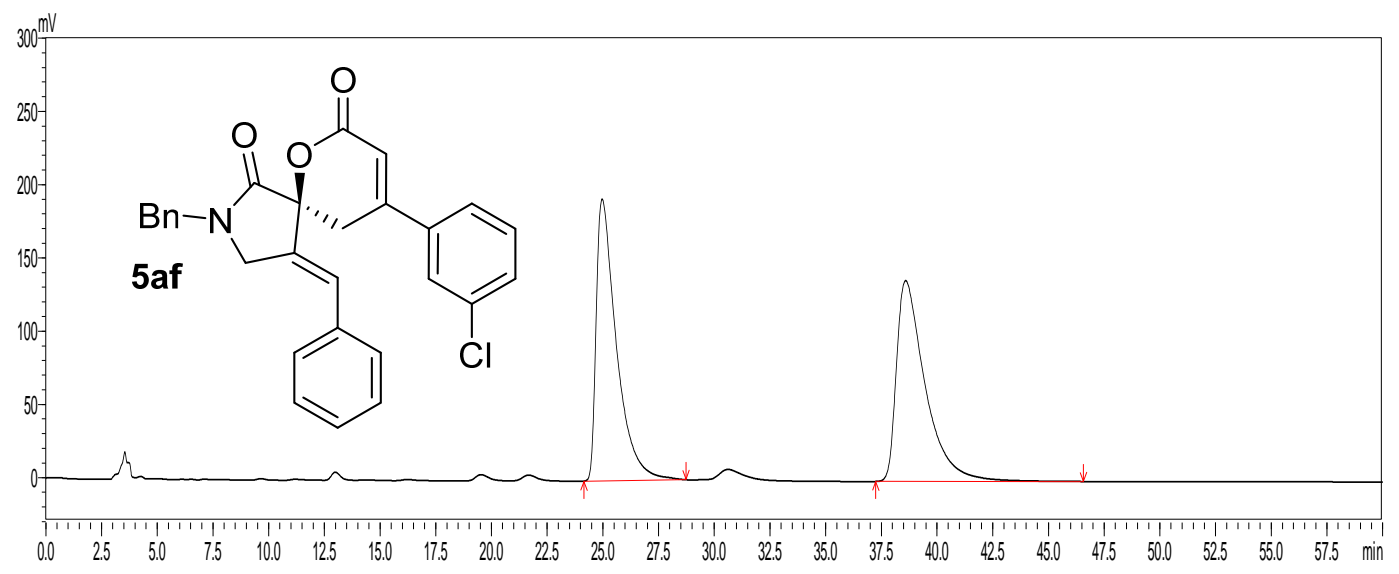

\begin{tabular}{|l|l|l|l|}
\hline & Retention & Area & Area\% \\
\hline 1 & 24.969 & 12127259 & 49.653 \\
\hline 2 & 38.590 & 12296619 & 50.347 \\
\hline
\end{tabular}

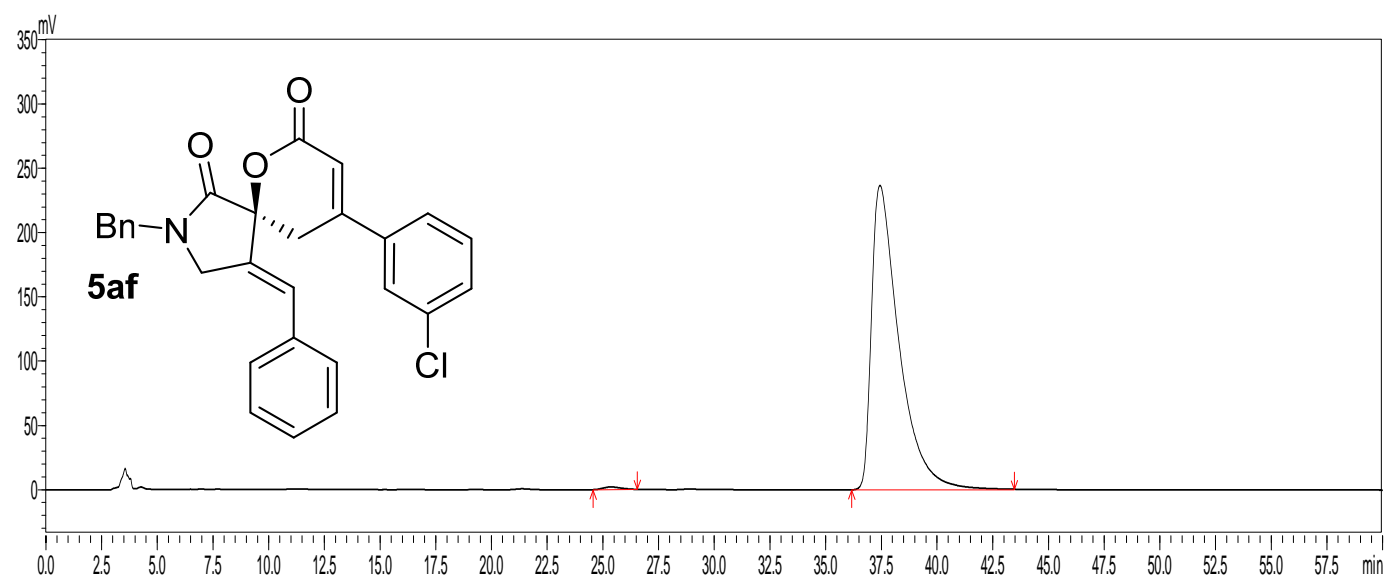

\begin{tabular}{|l|l|l|l|}
\hline & Retention & Area & Area\% \\
\hline 1 & 25.368 & 117141 & 0.571 \\
\hline 2 & 37.436 & 20380745 & 99.429 \\
\hline
\end{tabular}




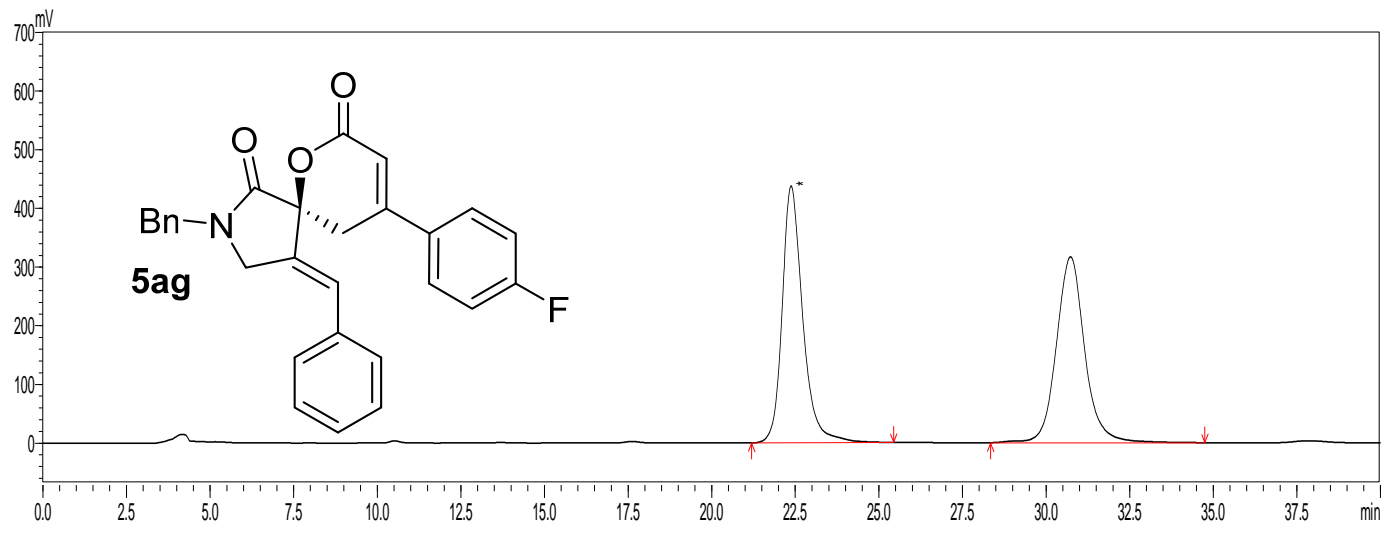

\begin{tabular}{|l|l|l|l|}
\hline & Retention & Area & Area\% \\
\hline 1 & 22.374 & 18723768 & 49.999 \\
\hline 2 & 30.732 & 18724430 & 50.001 \\
\hline
\end{tabular}

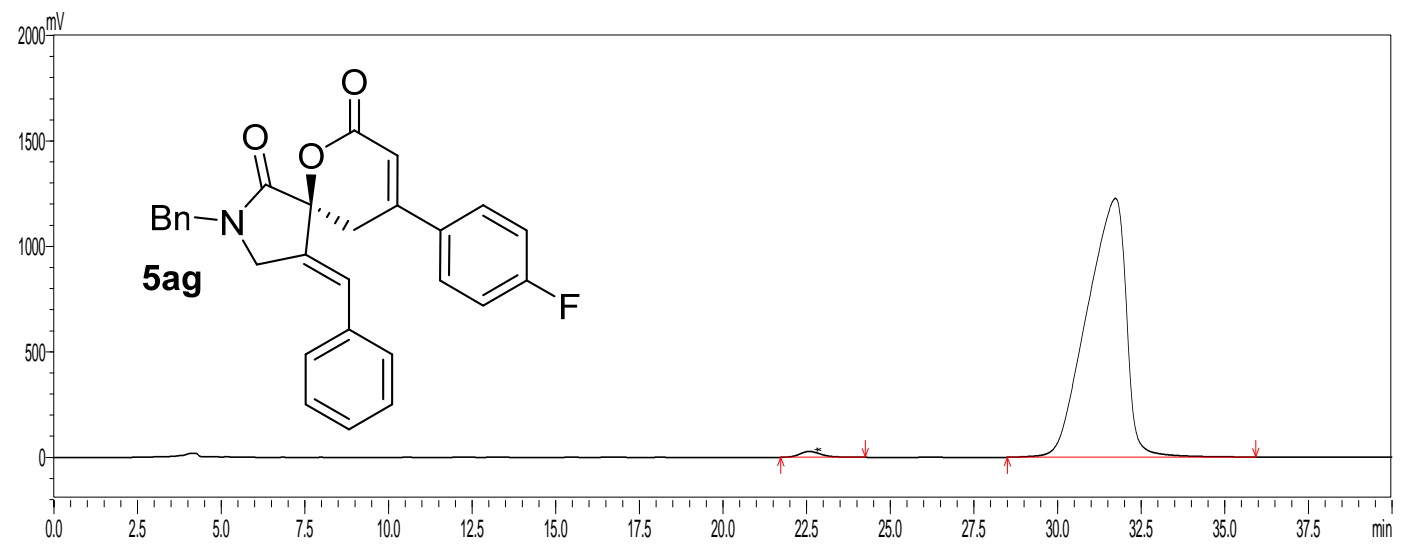

\begin{tabular}{|l|l|l|l|}
\hline & Retention & Area & Area\% \\
\hline 1 & 22.579 & 1253041 & 1.238 \\
\hline 2 & 31.731 & 99970095 & 98.762 \\
\hline
\end{tabular}

S159 


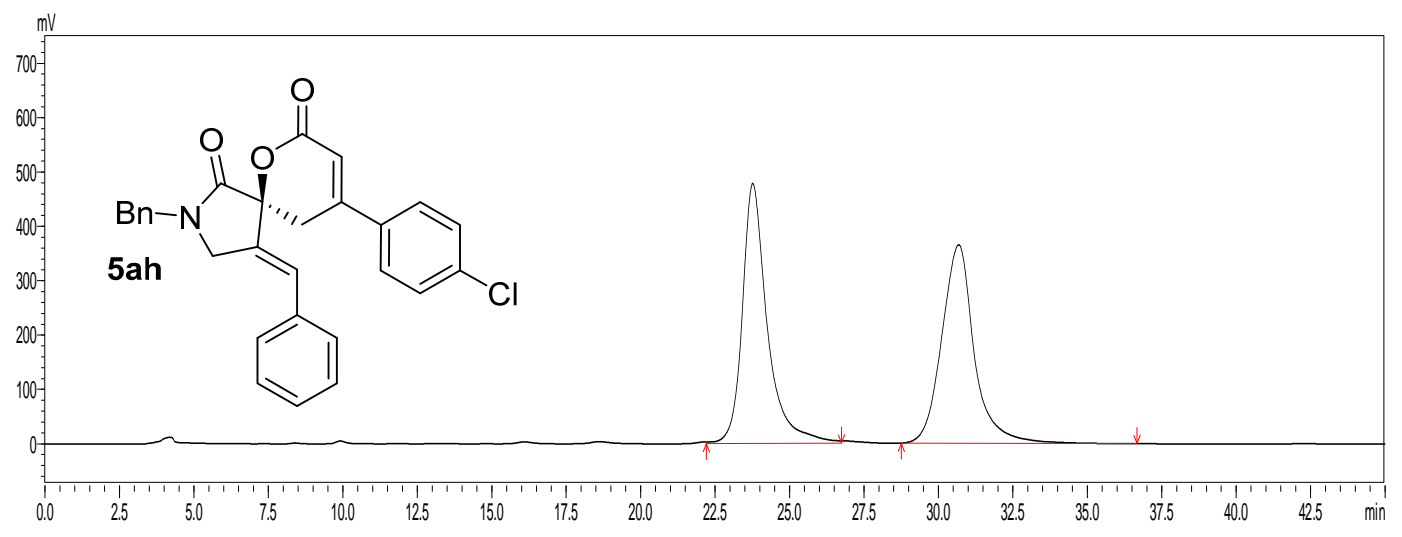

\begin{tabular}{|l|l|l|l|}
\hline & Retention & Area & Area\% \\
\hline 1 & 23.765 & 28000184 & 50.259 \\
\hline 2 & 30.681 & 27711228 & 49.741 \\
\hline
\end{tabular}

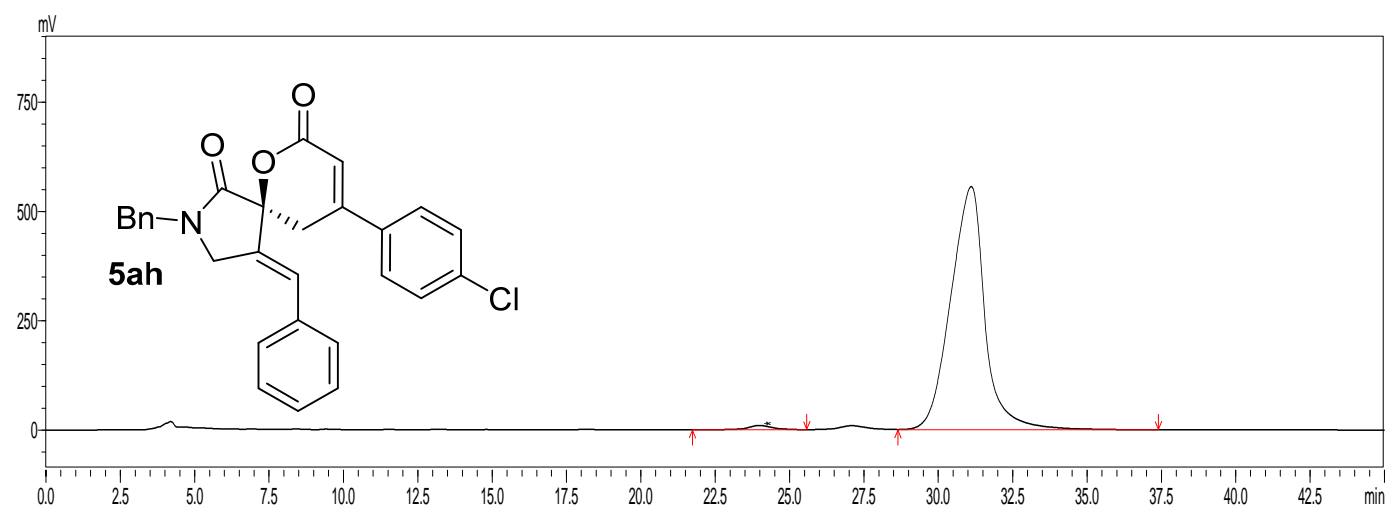

\begin{tabular}{|l|l|l|l|}
\hline & Retention & Area & Area\% \\
\hline 1 & 23.985 & 645480 & 1.424 \\
\hline 2 & 31.107 & 44674995 & 98.576 \\
\hline
\end{tabular}




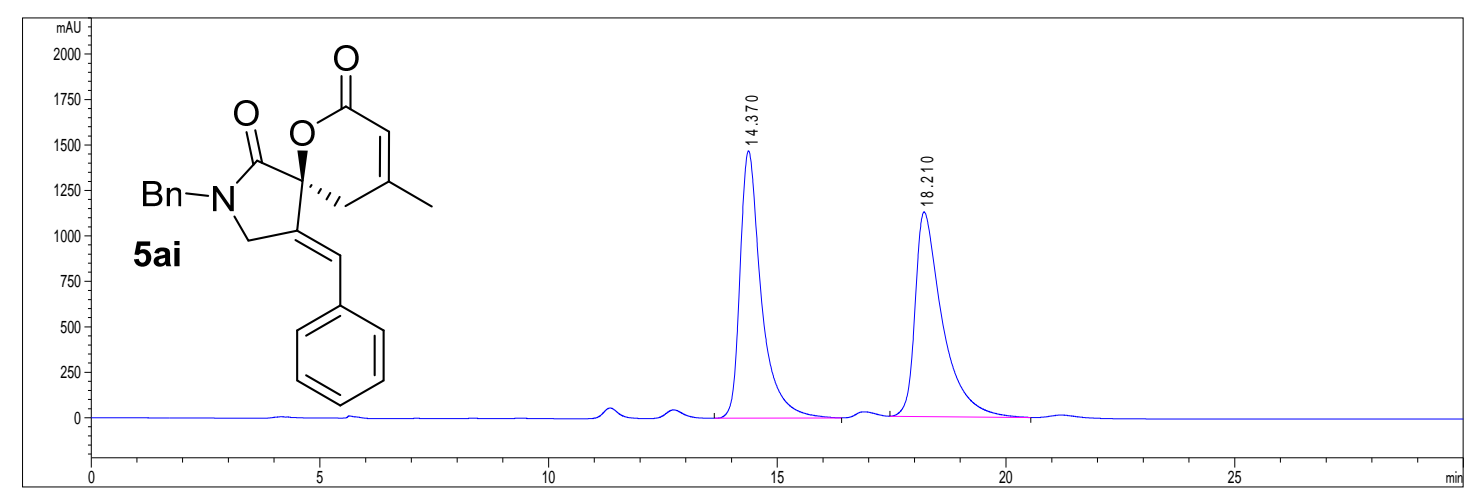

\begin{tabular}{|l|l|l|l|}
\hline & Retention & Area & Area\% \\
\hline 1 & 14.370 & $4.77213 \mathrm{e} 4$ & 50.4785 \\
\hline 2 & 18.210 & $4.68166 \mathrm{e} 4$ & 49.5215 \\
\hline
\end{tabular}

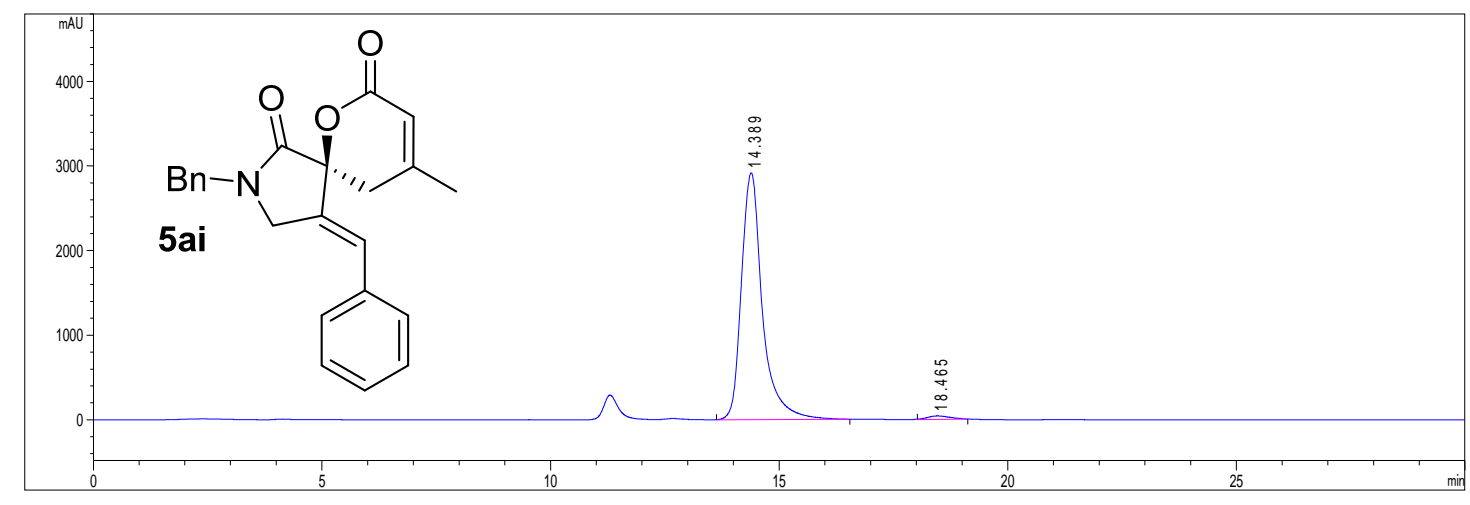

\begin{tabular}{|l|l|l|l|}
\hline & Retention & Area & Area\% \\
\hline 1 & 14.389 & $9.32452 \mathrm{e} 4$ & 98.5641 \\
\hline 2 & 18.465 & 1358.39502 & 1.4359 \\
\hline
\end{tabular}




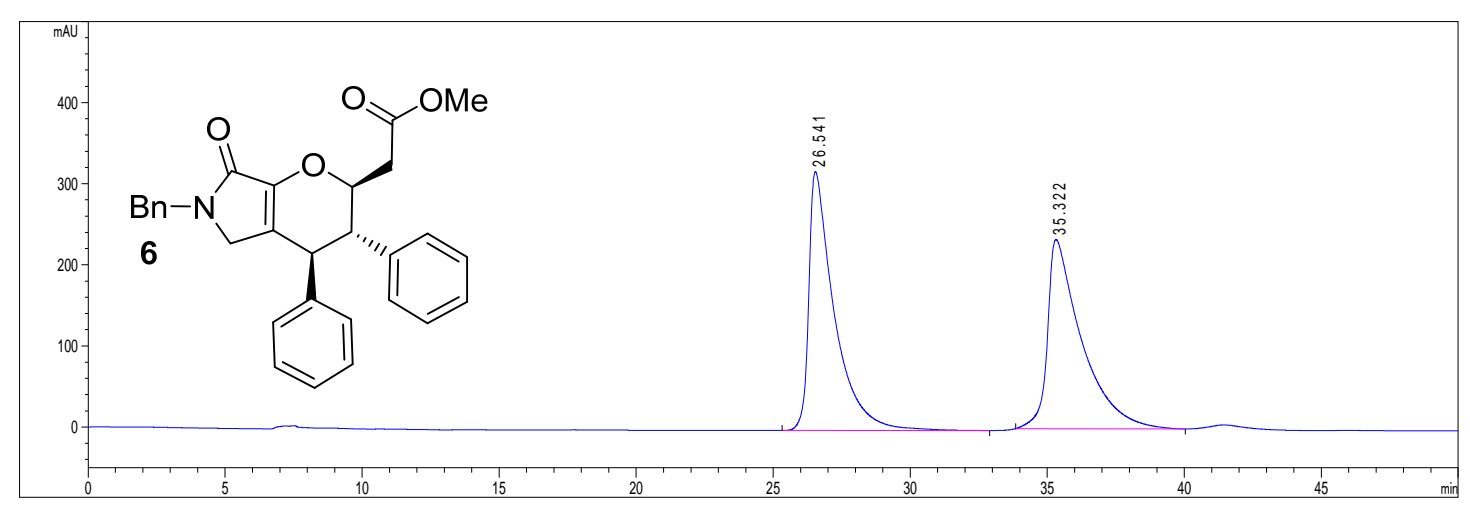

\begin{tabular}{|l|l|l|l|}
\hline & Retention & Area & Area $\%$ \\
\hline 1 & 26.541 & $2.05751 \mathrm{e} 4$ & 50.4093 \\
\hline 2 & 35.322 & $2.02409 \mathrm{e} 4$ & 49.5907 \\
\hline
\end{tabular}

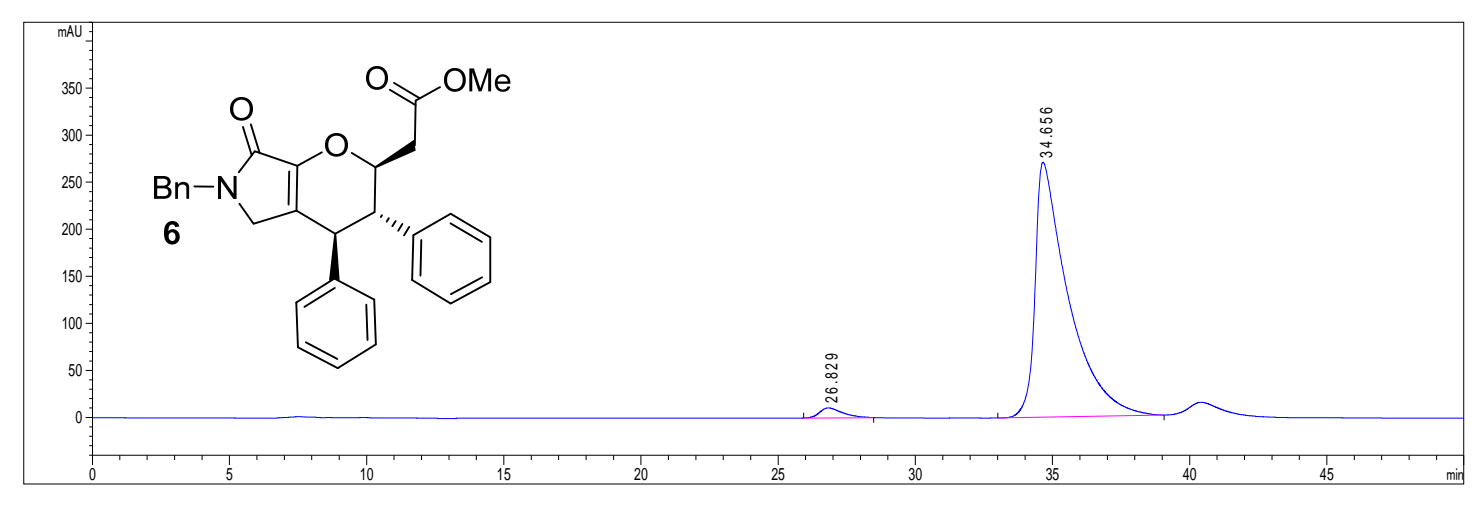

\begin{tabular}{|l|l|l|l|}
\hline & Retention & Area & Area $\%$ \\
\hline 1 & 26.829 & 624.86670 & 2.6646 \\
\hline 2 & 34.656 & $2.28260 \mathrm{e} 4$ & 97.3354 \\
\hline
\end{tabular}




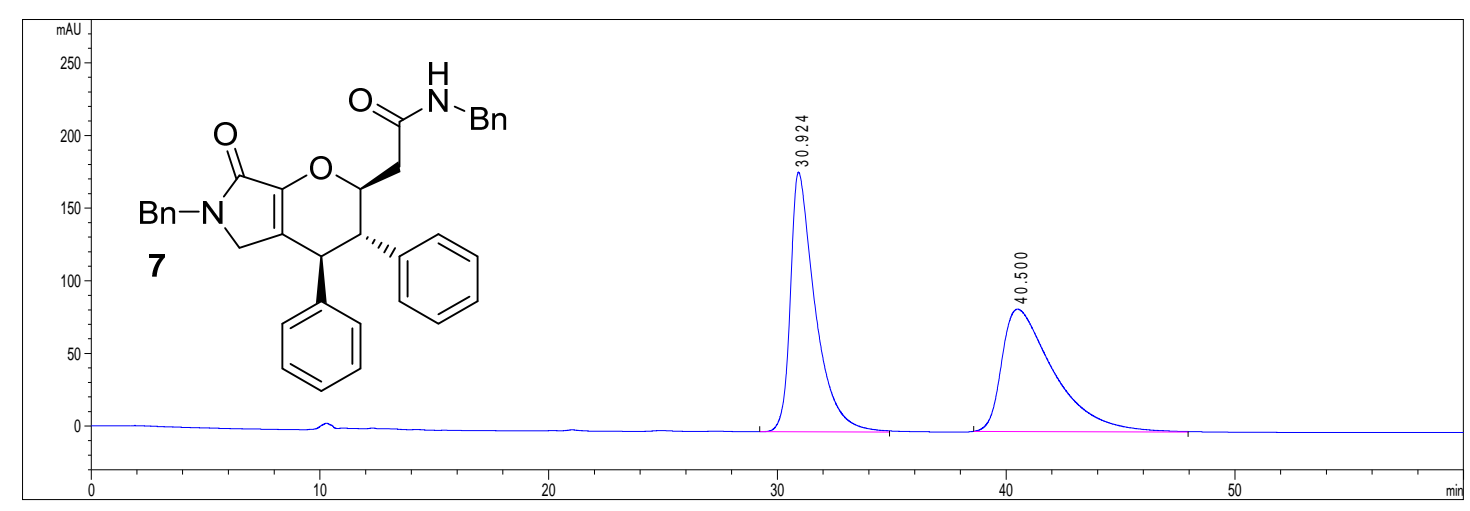

\begin{tabular}{|l|l|l|l|}
\hline & Retention & Area & Area\% \\
\hline 1 & 30.924 & $1.35180 \mathrm{e} 4$ & 50.3675 \\
\hline 2 & 40.500 & $1.33208 \mathrm{e} 4$ & 49.6325 \\
\hline
\end{tabular}

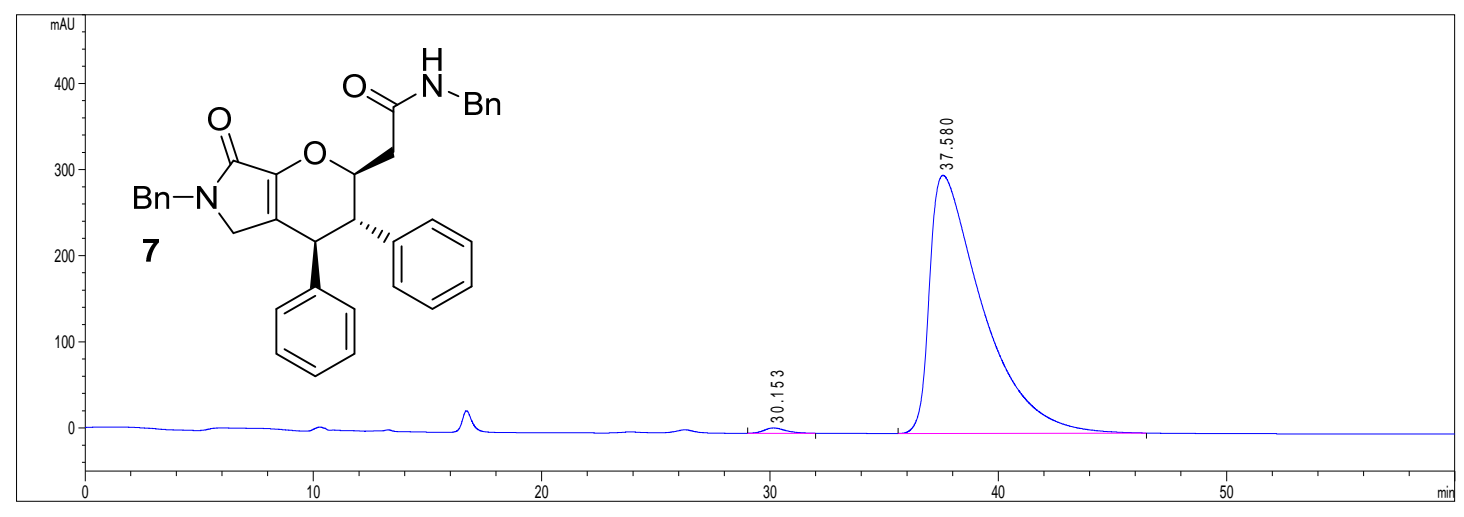

\begin{tabular}{|l|l|l|l|}
\hline & Retention & Area & Area\% \\
\hline 1 & 30.153 & 456.03641 & 0.9337 \\
\hline 2 & 37.580 & $4.83832 \mathrm{e} 4$ & 99.0663 \\
\hline
\end{tabular}




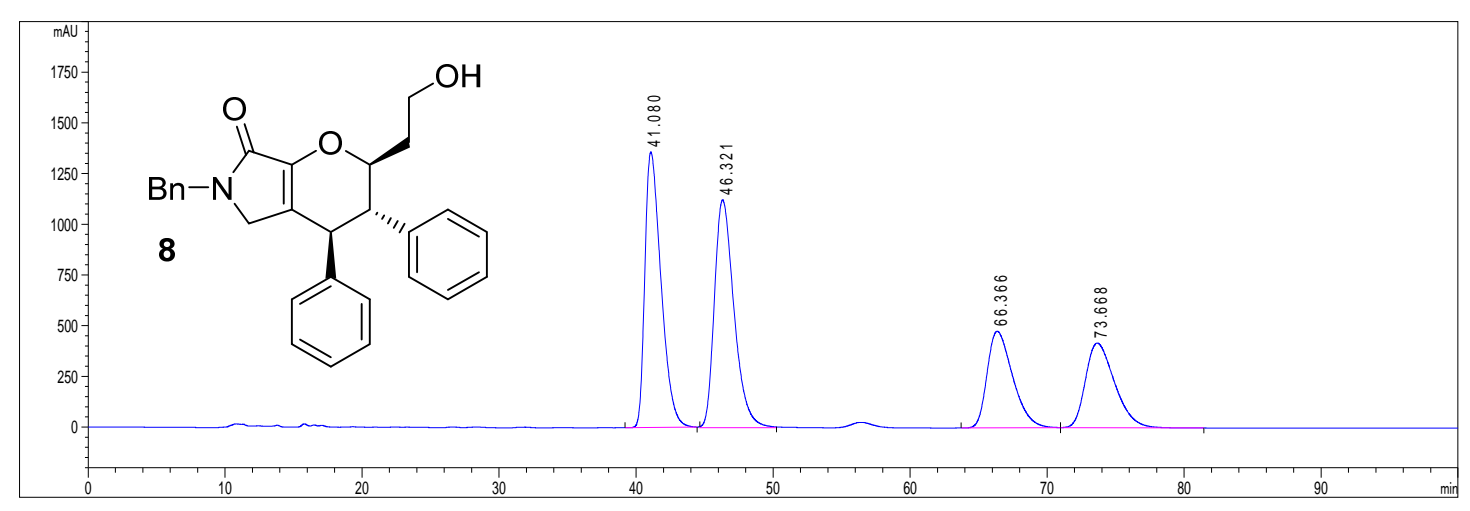

\begin{tabular}{|l|l|l|l|}
\hline & Retention & Area & Area\% \\
\hline 1 & 41.080 & $1.11204 \mathrm{e} 5$ & 32.1320 \\
\hline 2 & 46.321 & $1.11643 \mathrm{e} 5$ & 32.2589 \\
\hline 3 & 66.366 & $6.16486 \mathrm{e} 4$ & 17.8131 \\
\hline 4 & 73.668 & $6.15891 \mathrm{e} 4$ & 17.7959 \\
\hline
\end{tabular}

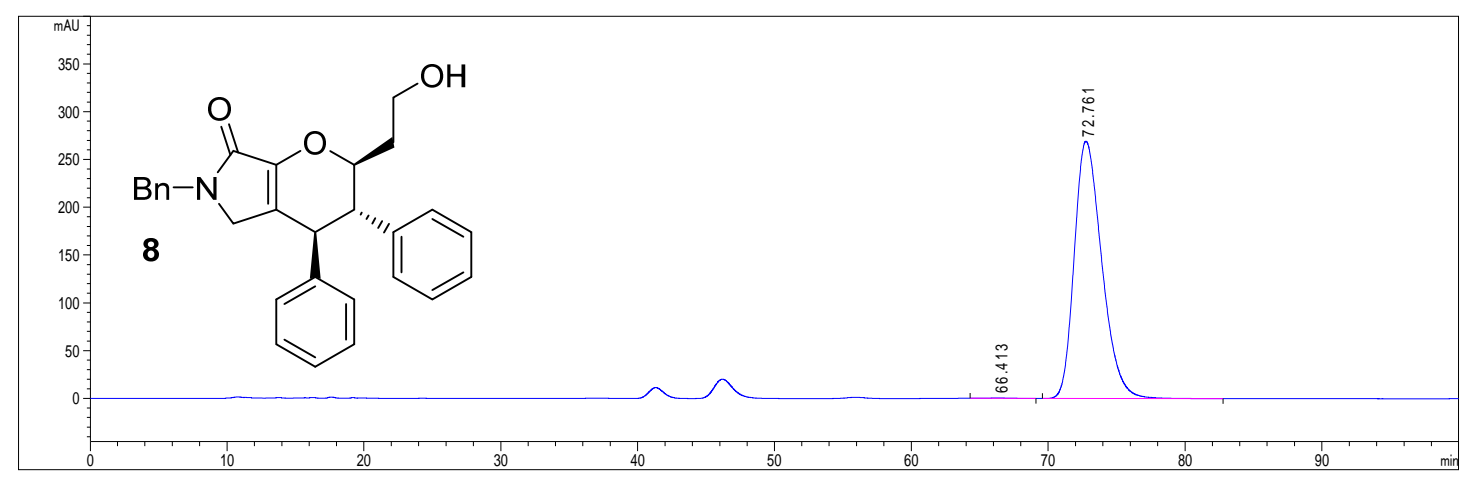

\begin{tabular}{|l|l|l|l|}
\hline & Retention & Area & Area\% \\
\hline 1 & 66.413 & 53.78944 & 0.1425 \\
\hline 2 & 72.761 & $3.76811 \mathrm{e} 4$ & 99.8575 \\
\hline
\end{tabular}




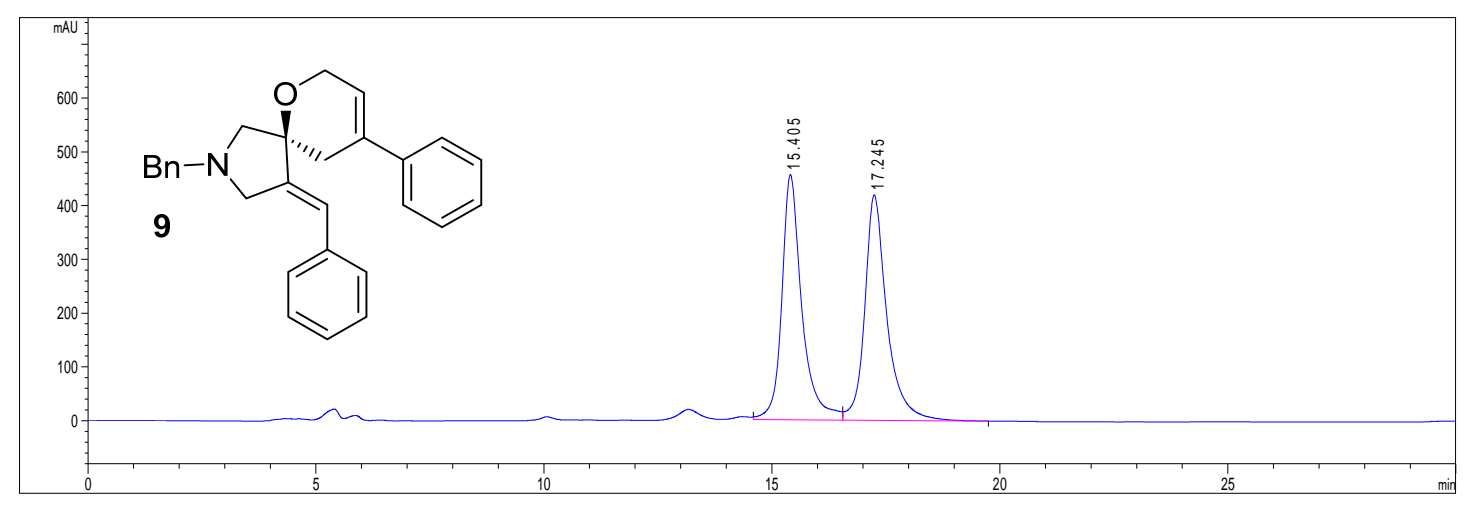

\begin{tabular}{|l|l|l|l|}
\hline & Retention & Area & Area $\%$ \\
\hline 1 & 15.405 & $1.39797 \mathrm{e} 4$ & 49.4807 \\
\hline 2 & 17.245 & $1.42731 \mathrm{e} 4$ & 50.5193 \\
\hline
\end{tabular}

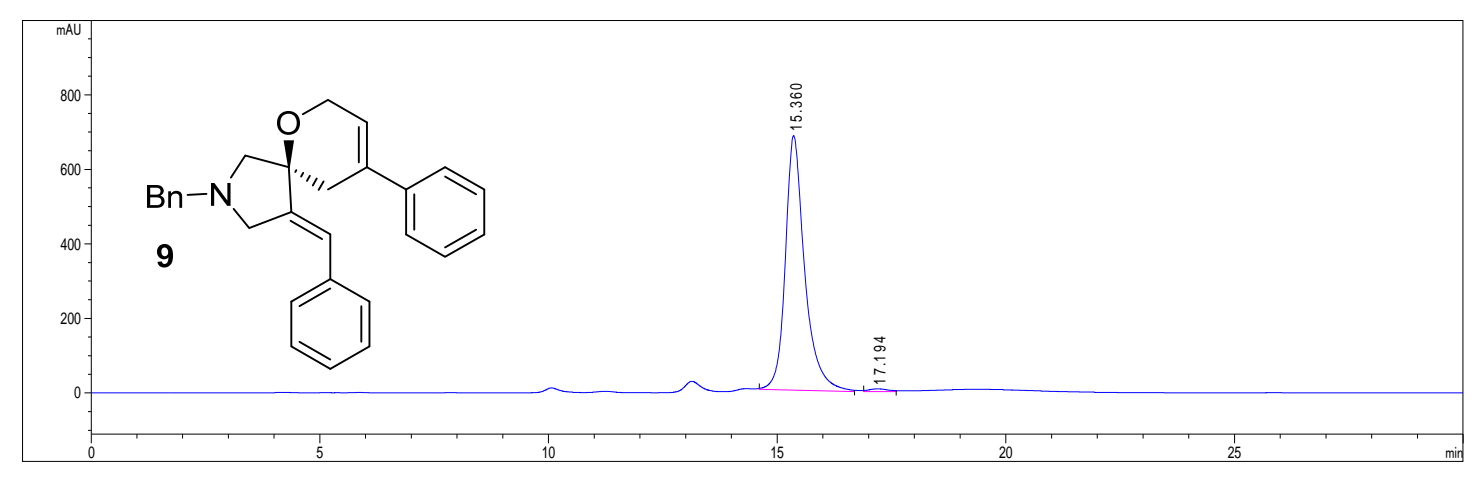

\begin{tabular}{|l|l|l|l|}
\hline & Retention & Area & Area\% \\
\hline 1 & 15.360 & $2.01710 \mathrm{e} 4$ & 98.9637 \\
\hline 2 & 17.194 & 211.21281 & 1.0363 \\
\hline
\end{tabular}

